archives-ouvertes

\title{
Populations et tendances démographiques des pays européens (1980-2010)
}

Alexandre Avdeev, Tatiana Eremenko, Joëlle Gaymu, Patrick Festy, Nathalie Le Bouteillec, Sabine Springer

\section{To cite this version:}

Alexandre Avdeev, Tatiana Eremenko, Joëlle Gaymu, Patrick Festy, Nathalie Le Bouteillec, et al.. Populations et tendances démographiques des pays européens (1980-2010). Population Paris, Institut National D'etudes Demographiques, 2011, Démographie des régions du monde: état et tendances, 66 (1), pp.9-133. https://www.cairn.info/revue-population-2011-1-page-9.htm . 10.3917/popu.1101.0009 . hal-02179006

\section{HAL Id: hal-02179006 \\ https://hal.archives-ouvertes.fr/hal-02179006}

Submitted on 10 Jul 2019

HAL is a multi-disciplinary open access archive for the deposit and dissemination of scientific research documents, whether they are published or not. The documents may come from teaching and research institutions in France or abroad, or from public or private research centers.
L'archive ouverte pluridisciplinaire HAL, est destinée au dépôt et à la diffusion de documents scientifiques de niveau recherche, publiés ou non, émanant des établissements d'enseignement et de recherche français ou étrangers, des laboratoires publics ou privés. 


\section{Alexandre AVDEEV * $\bullet$, Tatiana EREMENKo $\bullet$, Patrick FESTY $\bullet$, Joëlle GAYMU $\bullet$, Nathalie Le BOUTEILLEC ** $\bullet$, Sabine SPRINGER ${ }^{\circ}$}

\section{Populations et tendances démographiques des pays européens (1980-2010)}

L'Europe est la région du monde dont la démographie est la mieux étudiée et documentée. C'est en se fondant sur de longues observations de la situation démographique et des tendances de la reproduction de la population des pays européens que le concept général de révolution, ou de transition démographique, a été avancé (Rabinowicz, 1929 ; Landry, 1934) ${ }^{(1)}$. Les riches données concernant les populations européennes ont servi de base à la démographie historique, et notamment aux études sur la baisse séculaire de la fécondité et la transformation de la famille (Henry, 1953 ; Fleury et Henry, 1956 ; Goubert, 1960). Les statistiques détaillées de la mortalité selon les causes de décès dans les pays d'Europe ont permis de formuler le concept de transition épidémiologique (Omran, 1971). Enfin, l'analyse des tendances démographiques des années 1980-1990 a engendré l'idée d'une « seconde transition démographique », dont les apports scientifiques sont encore loin d'être taris (Lesthaeghe et van de Kaa, 1986 ; van de Kaa, 1987 et 2003 ; Lesthaeghe et Surkyn, 2004).

C'est aussi dans les pays européens que le système moderne de la statistique démographique a été forgé - offrant dans certains cas des séries d'indicateurs démographiques depuis le milieu du XVIII siècle -, que les principes des recensements généraux de population ont été mis au point, et que les premières classifications des causes de décès et des maladies ont vu le jour.

(1) Léon Rabinowicz a utilisé pour la première fois la locution "révolution démographique » en 1929 pour désigner les changements démographiques des pays développés, expression que reprendra Adolphe Landry en 1934.

* Université Paris I Panthéon Sorbonne, Institut de démographie.

- Institut national d'études démographiques, Paris.

** Université de Picardie Jules Verne, Curapp.

${ }^{\circ}$ Université Montesquieu-Bordeaux IV-IEDUB.

Correspondance : Patrick Festy, Institut national d'études démographiques, 133 bd Davout, 75980 Paris Cedex 20, tél : 33 (0)1 560622 01, courriel : festy@ined.fr 
Toutefois, l'étude de la population européenne dans les décennies récentes est marquée par :

- l'imprécision des lisières géographiques de la région et de ses sousensembles, ainsi que par la variabilité des frontières politiques et administratives des pays européens ;

- l'hétérogénéité politique, économique et démographique du continent ;

- l'impact des bouleversements politiques au moment même où les comportements démographiques de la population connaissaient des évolutions profondes.

Nous préciserons donc dans un premier temps le cadre de l'étude, avant de passer en revue les mouvements globaux ayant affecté la population européenne, puis plus en détail, la fécondité, la mortalité, le vieillissement et les migrations internationales. Un chapitre annexe présente rapidement les principales sources statistiques mobilisées pour ce travail et fait le point sur les recensements et les grandes enquêtes comparatives européennes.

\section{Espace et période d'étude}

\section{Choix d'une période d'étude}

Récemment, Alain Monnier (2006) a dressé un bilan du développement démographique de l'Europe au $\mathrm{XX}^{\mathrm{e}}$ siècle en distinguant trois périodes. Le milieu des années 1960 marque la fin de la transition démographique classique, lorsque la baisse de la fécondité se réalise dans le cadre de la famille traditionnelle basée sur un mariage universel et difficile à rompre. Ensuite, jusqu'à la fin des années 1980, le modèle traditionnel est en crise et un nouveau modèle apparaît, avec l'assouplissement de plusieurs contraintes juridiques pesant sur les comportements démographiques (autorisation et simplification de la procédure du divorce, accès libre à l'avortement et à la contraception). Enfin, dès la fin des années 1980, commence une période de consolidation et d'institutionnalisation des nouvelles formes conjugales et familiales (stabilisation et soutien législatif des formes nouvelles d'unions, concordance entre relations conjugales et parentales hors du mariage traditionnel). Ces changements fondamentaux sont accompagnés par une diminution importante de la mortalité, qui a aussi des conséquences sur la structure du cycle familial et les relations intergénérationnelles. Cette périodisation rejoint le concept de seconde transition démographique élaboré par R. Lesthaeghe et D. J. van de Kaa en 1986. Nous nous attachons ici à la phase la plus récente ${ }^{(2)}$, celle de la consolidation du modernisme démographique de l'Europe selon l'expression d'Alain Monnier.

(2) Les périodes antérieures sont connues grâce à de nombreuses études, le plus souvent publiées dans la revue Population. La chronique de la démographie européenne y a fait l'objet d'une série de publications, débutée en 1949 par un article de L. Henry sur l'évolution démographique de l'Europe entre 1938 et 1947. Ensuite, la chronique démographique de l'Europe et des pays développés a périodiquement été réalisée par R. Pressat (jusqu'en 1973), J.-N. Biraben (1975-1978), A. Monnier (1979-1999, avec la collaboration de C. de Guibert-Lantoine de 1992 à 1997) et par J.-P. Sardon (2000-2007). 
L'histoire de l'Europe durant ces trente dernières années est également diversifiée, et peut elle aussi être divisée en trois périodes de durées presque égales. La première décennie, de 1980 à 1990, est marquée par le déclin général du socialisme totalitaire et de l'économie centralisée dans les pays d'Europe de l'Est. À l'Ouest, les dernières dictatures avaient disparu dans les années 1970 : en 1974 pour la Grèce et le Portugal et en 1975 pour l'Espagne. En Europe de l'Est, de la fin des années 1980 au début des années 1990, le système du socialisme totalitaire s'effondre (révolutions de velours), aboutissant à la constitution de nouveaux États nationaux indépendants, plus ou moins homogènes sur le plan ethnique. Plusieurs pays sont effacés de la carte politique de l'Europe : la République démocratique d'Allemagne rejoint la République fédérale d'Allemagne en 1990 ; l'URSS, la Tchécoslovaquie et la Yougoslavie font place à treize nouveaux États aux côtés desquels surgissent quelques territoires avec un statut politique flou, leur indépendance n'étant pas reconnue ou reconnue partiellement par les États membres de l'Organisation des Nations unies (Transnistrie, Abkhazie, Kosovo, Ossétie du Sud).

Il en résulte des redistributions territoriales de la population, des ruptures dans les tendances démographiques et des flux migratoires spécifiques. Cependant, la dynamique migratoire dans les années 1990 ne change pas seulement à cause des conflits, mais aussi à la suite de la libéralisation des régimes politiques et de la détérioration de la situation économique dans les pays d'Europe de l'Est anciennement socialistes. Les nouveaux flux de migrations de travail se dirigent de la Roumanie, de la Pologne et des nouveaux pays balkaniques vers l'Occident ; de l'Ukraine et de la Moldavie vers la Russie et la Pologne, du Caucase et de l'Asie centrale vers la Russie. Ils rejoignent et renforcent les flux européens « traditionnels » des anciennes colonies vers les métropoles, mais leur potentiel est limité. Vers la fin des années 1990, ces mouvements perdent en importance, et les nouveaux États d'Europe de l'Est connaissent croissance économique, création de nouveaux emplois et amélioration des conditions de vie. Pendant qu'à l'est et au centre de l'Europe on assiste à la formation de l'économie de marché et à la démocratisation des formes de gouvernance politique, en Europe occidentale les processus d'intégration économique et politique s'intensifient. C'est pourquoi la décennie 1990-2000 peut être vue comme une période de transition pour l'ensemble du continent.

Enfin, la première décennie du $\mathrm{XXI}^{\mathrm{e}}$ siècle est une période de stabilisation des nouvelles structures politiques et économiques à l'Est aussi bien qu'à l'Ouest, accompagnée par le renforcement des processus d'intégration en raison de l'élargissement de l'Union européenne vers l'est.

\section{Espace géopolitique et histoire}

\section{Un contour géographique variable}

Aujourd'hui, l'Europe est géographiquement définie comme la partie occidentale du continent eurasiatique avec les îles britanniques et l'Islande. Ses 
frontières continentales au nord, à l'ouest et au sud sont naturellement définies par les littoraux arctique, atlantique et méditerranéen. En revanche à l'est, la frontière naturelle entre l'Europe et l'Asie, définie selon le versant est des montagnes de l'Oural, reste contestable.

Encadré : liste des pays d'Europe par région et de leurs codes ISO

\begin{tabular}{|c|c|c|c|c|c|}
\hline \multicolumn{2}{|c|}{ Nord } & \multicolumn{2}{|l|}{ Sud } & \multicolumn{2}{|c|}{ Centre } \\
\hline Danemark & DK & Albanie & $A L$ & Bulgarie & $B G$ \\
\hline Finlande & $\mathrm{FI}$ & Bosnie-Herzégovine & BA & Hongrie & $\mathrm{HU}$ \\
\hline Islande & IS & Chypre & CY & Pologne & $\mathrm{PL}$ \\
\hline Norvège & NO & Croatie & $H R$ & Rép. tchèque & $C Z$ \\
\hline Suède & SE & Espagne & ES & Roumanie & RO \\
\hline \multicolumn{2}{|c|}{ Ouest } & Grèce & $\mathrm{GR}$ & Slovaquie & SK \\
\hline Allemagne & $\mathrm{DE}$ & Italie & IT & \multicolumn{2}{|c|}{ Est } \\
\hline Autriche & AT & Macédoine & MK & Belarus & BY \\
\hline Belgique & $\mathrm{BE}$ & Malte & MT & Estonie & $\mathrm{EE}$ \\
\hline France & $\mathrm{FR}$ & Monténégro & $\mathrm{ME}$ & Lettonie & LV \\
\hline Irlande & $\mathrm{IE}$ & Portugal & PT & Lituanie & LT \\
\hline Luxembourg & $\mathrm{LU}$ & Serbie & RS & Moldavie & MD \\
\hline Pays-Bas & $\mathrm{NL}$ & Slovénie & $\mathrm{SI}$ & Russie & $\mathrm{RU}$ \\
\hline Royaume-Uni & $\mathrm{GB}$ & & & Ukraine & UA \\
\hline Suisse & $\mathrm{CH}$ & & & & \\
\hline
\end{tabular}

La frontière politique de l'Europe, de même que ses frontières culturelles et linguistiques, ne coïncide pas non plus avec sa frontière géographique. Actuellement, selon la régionalisation officielle du monde de l'Organisation des Nations unies, l'Europe est partagée dans le sens de l'aiguille d'une montre entre quatre sous-régions : le Nord, l'Est, le Sud et l'Ouest ${ }^{(3)}$. À cette régionalisation basée sur des critères purement géographiques, qui ne correspond pas à l'histoire politique et culturelle de l'Europe, nous avons préféré un découpage en cinq régions, de taille inégale mais de composition plus homogène (encadré et figure 1):

- la région Nord comprend les cinq pays nordiques (Danemark, Finlande, Islande, Norvège et Suède) ;

- la région Est englobe les sept anciennes républiques de l'Union soviétique ;

(3) Pour l'ONU, l'Europe inclut la Fédération de Russie, avec une surface de 17 millions $\mathrm{km}^{2}$ dont seuls 3,9 sont situés géographiquement en Europe. En revanche, quatre pays partiellement situés en Europe ne sont pas considérés par l'ONU comme européens : le Kazakhstan, la Turquie, la Géorgie et l'Azerbaïdjan. Assimilés à l'Asie occidentale, les trois derniers sont néanmoins membres du Conseil de l'Europe. Nous avons suivi l'ONU sur ces points. Par manque de données détaillées suffisamment fiables, nous avons exclu de nos analyses les pays les moins peuplés, comme Andorre, Monaco, le Lichtenstein, Saint-Marin et le Vatican (voir. annexe p. 101). Enfin, quelques pays européens possèdent des territoires d'outre-mer généralement de taille limitée, avec des populations peu nombreuses. 
- la région Centre comprend tous les autres pays anciennement socialistes situés au centre de l'Europe ;

- la région Sud inclut tous les pays méditerranéens ainsi que Chypre ${ }^{(4)}$ et le Portugal ;

- la région Ouest est composée de tous les pays restants.

Dans la suite de nos analyses, selon la disponibilité des données, nous avons inclus ou exclu trois pays de la région Est : la Russie, l'Ukraine et le Bélarus.

Figure 1. Carte d'Europe et classement des pays en 5 régions

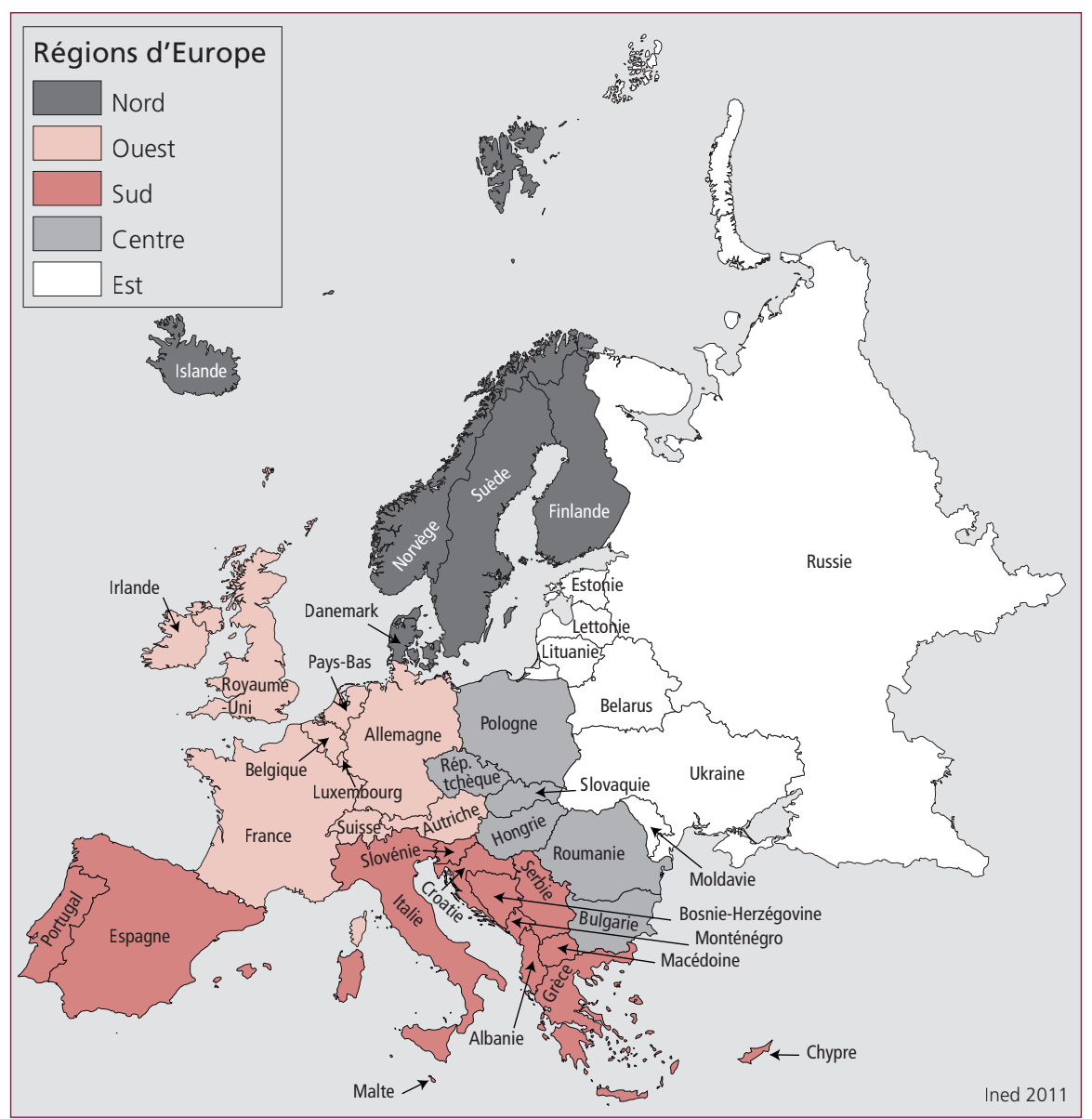

(4) La république de Chypre est située par l'ONU en Asie occidentale, bien qu'en 2004 elle ait adhéré à l'Union européenne et en 2008 à la zone euro. 
Tableau 1. Pays et revenu national brut par habitant (\$) en 2008 pour les cinq régions d'Europe

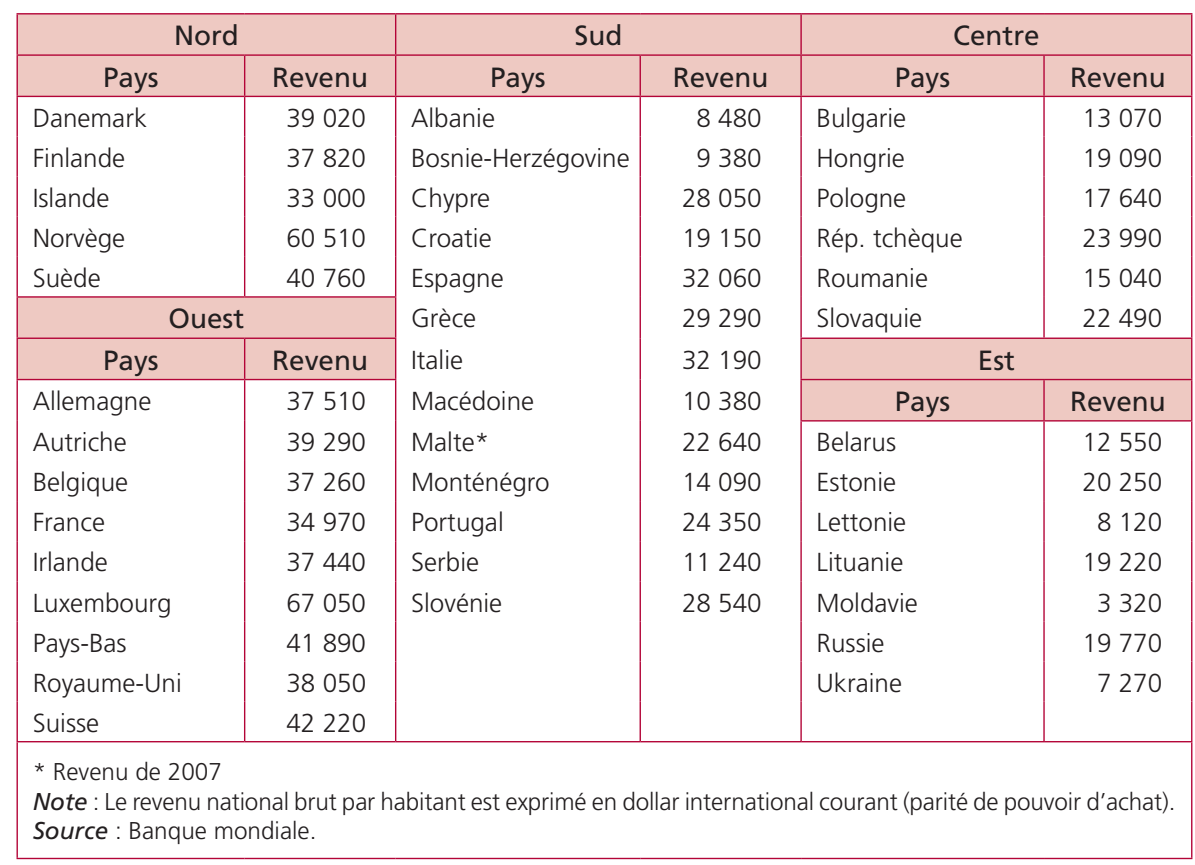

Quant à l'Union européenne, elle rassemble 27 pays avec une population de presque 498 millions d'habitants, parmi lesquels 16 pays (330 millions d'habitants) ont la même monnaie et forment la zone euro.

\section{Les frontières nationales ont évolué}

Par sa structure politique interne, l'Europe représente un conglomérat complexe et historiquement instable. Au cours des deux derniers siècles, la géographie politique de l'Europe s'est constamment transformée. La fin du $\mathrm{XX}^{\mathrm{e}}$ siècle est marquée par le fractionnement de l'espace : la dissolution de l'Union soviétique en décembre 1991, plus ou moins sans conflits ; la séparation pacifique de la Tchécoslovaquie en 1993 en Slovaquie et République tchèque ; la désintégration longue et douloureuse de la Yougoslavie, dont la Croatie et la Macédoine ${ }^{(5)}$ se sont séparées en 1991, suivies en 1992 par la Slovénie et la Bosnie-Herzégovine. Constitués en Communauté d'États en 2003, la Serbie et le Monténégro ont laissé place à deux États indépendants en 2006. Mais l'épopée de la construction de la carte politique des Balkans continue jusqu'à nos jours en raison de la situation du Kosovo, reconnu par seulement 70 États membres de l'ONU après avoir proclamé unilatéralement son indépendance de la République de Serbie en 2008.

(5) Depuis 1993, la dénomination officielle est l'Ancienne république yougoslave de Macédoine. 
Compte tenu de cette histoire politique mouvementée, l'Europe, souvent considérée comme « l'ancien monde », représente de fait l'une des plus jeunes, voire la plus jeune région géopolitique du monde contemporain, région dont la frontière générale reste encore très floue.

De façon à assurer le maximum de cohérence dans cette chronique démographique de l'Europe depuis 1980, son espace géographique et sa population ont été ramenés dans les limites des frontières des pays formant la région de l'Europe selon la définition de l'ONU, en y ajoutant la république de Chypre. Pour davantage de pertinence, la présentation du mouvement de population et des tendances démographiques a été faite dans les frontières actuelles des États européens. Ainsi, pour la période 1980-1990, les statistiques nationales des pays disparus comme la Tchécoslovaquie, la Yougoslavie et l'URSS ont été fractionnées dans le respect des frontières de leurs anciennes provinces autonomes, unités fédérales, ou des républiques d'union, devenues de nouveaux États indépendants. Concernant la même période, les statistiques de la RDA et de la RFA ont été fusionnées afin de ramener les tendances démographiques de la République fédérale d'Allemagne dans ses frontières actuelles.

\section{Hétérogénéité socioéconomique}

Comprendre la situation démographique de l'Europe et expliquer son évolution suppose de prendre en considération non seulement une dimension géopolitique, mais aussi économique. Le niveau de bien-être et de potentiel économique des pays, traduit par le revenu national brut par habitant, en est une bonne illustration (tableau 1). Sur ce plan, l'Europe dans son ensemble est l'une des plus riches régions du monde. Aujourd'hui, avec une population de 734 millions d'habitants, soit un dixième de la population mondiale, l'Europe représente plus du quart du revenu mondial annuel. Son revenu par habitant est supérieur à 30000 dollars de parité de pouvoir d'achat (\$ PPA), moins que celui des États-Unis (47 500 \$ PPA), mais largement supérieur à la moyenne mondiale (environ 10000 \$ PPA).

Le revenu par habitant en Europe varie beaucoup d'un pays à l'autre. Entre le pays le plus riche (Luxembourg) et le plus pauvre (Moldavie) le rapport est de 1 à 20. Cinq pays ont un revenu par habitant inférieur à 10000 \$ PPA; les seize pays (dont la Russie) où cet indicateur est inférieur à 20000 \$ PPA regroupent $42 \%$ de la population européenne. Les pays les plus riches se situent sur la périphérie nord-ouest de l'Europe ; les géants démographiques de l'Europe occidentale et méridionale sont dans une position intermédiaire et les pays les plus pauvres se trouvent dans les Balkans (Grèce non comprise) et à l'Est de l'Europe. 


\section{La croissance de la population de l'Europe et ses composantes entre 1980 et 2009}

\section{Aperçu général de 1950 à 2009}

Depuis la fin de la deuxième guerre mondiale, la population européenne dans son ensemble a achevé sa transition démographique. Dans les années 1950, le taux d'accroissement annuel de la population de l'Europe oscillait entre $10 \%$ et $11 \%$ (figure 2A). Il a connu ensuite une diminution continue, pour devenir nul en 1995 et 1996. Jusque-là, la croissance était essentiellement assurée par l'excédent naturel. Mais la migration est devenue de plus en plus importante et, à partir du milieu des années 1990, elle compense une grande partie des pertes de population liées à l'excédent des décès sur les naissances. Entre 1997 et 2001 la population de l'Europe a diminué de 2,08 millions d'habitants ${ }^{(6)}$, mais sans l'apport migratoire la diminution aurait été plus de deux fois supérieure (4,86 millions). En 2002-2008, la population de l'Europe retrouve une légère croissance (2,5\%o en 2007 et 2008), complètement assurée par la migration.

Avant la fin des années 1970, les contrastes entre pays étaient stables, voire même en légère diminution, si on en juge par les quartiles inférieurs et supérieurs de la distribution autour de la médiane (figure 2B). Entre les deux, lécart interquartile, qui était de sept points (entre $6 \%$ et $13 \%$ ) dans la première moitié des années 1950, était réduit à cinq points (entre $5 \%$ ot $10 \%$ o) vingt ans plus tard, témoignant d'une légère convergence entre les pays européens. Depuis, les écarts se sont au contraire creusés de façon d'autant plus remarquable que la croissance reculait dans son ensemble. Dans la première moitié des années 2000, il y a huit points d'écart entre les quartiles inférieur et supérieur (entre $-2 \%$ et $+6 \%$ ) avec une médiane sensiblement réduite. Au début des années 1990, un petit nombre de pays ont connu une baisse importante de leur croissance démographique au lendemain de la chute du mur de Berlin, entraînant une forte diminution du premier décile de la distribution des taux d'accroissement, phénomène qui s'est partiellement résorbé par la suite. Une timide diminution de l'écart interquartile se dessine même à la fin des années 2000, trop récente pour être analysée.

Le recul général de la croissance démographique est, on l'a vu, largement dominé par la diminution de l'excédent des naissances sur les décès. Pour l'ensemble du continent, le solde négatif apparaît au début des années 1990 (figure 2C) et se développe ensuite rapidement, mais ce mouvement est dominé par la masse démographique de la Russie. La médiane des pays reste quant à elle toujours légèrement positive, même lors du déficit le plus important. La dispersion, mesurée par l'écart interquartile, diminue entre le début des années 1950 (neuf points de $7 \%$ à $16 \%$ o) et le début des années 1970 (cinq points de

(6) Il faut noter que pour cette période, le résultat de l'addition de l'excédent naturel (naissances moins décès) au solde migratoire ne correspond pas forcément à la variation de l'effectif total à cause de l'ajustement statistique. Concernant la définition de cet indicateur, voir « L'évolution démographique récente en Europe, 2004 » Édition du Conseil de l'Europe, Strasbourg, 2005, p. 120. 
$4 \%$ à $9 \%$ o), imposant son évolution à la dispersion de la croissance démographique dans son ensemble. Au-delà, il n'y a plus rien de systématique, si bien qu'au début des années 2000, la dispersion des taux d'accroissement naturel n'est pas différente de ce qu'elle était au début des années 1970 (cinq points à nouveau de $-2 \%$ à $+3 \%$ ). Ce maintien de la dispersion est toutefois partiellement trompeur, car les taux ont fortement diminué entre temps.

Figure 2. Évolution de l'accroissement de la population et de ses composantes dans l'ensemble de l'Europe et dans les pays européens depuis 1950

A. Taux brut (axe à droite) et composantes de l'accroissement de la population européenne (axe à gauche)

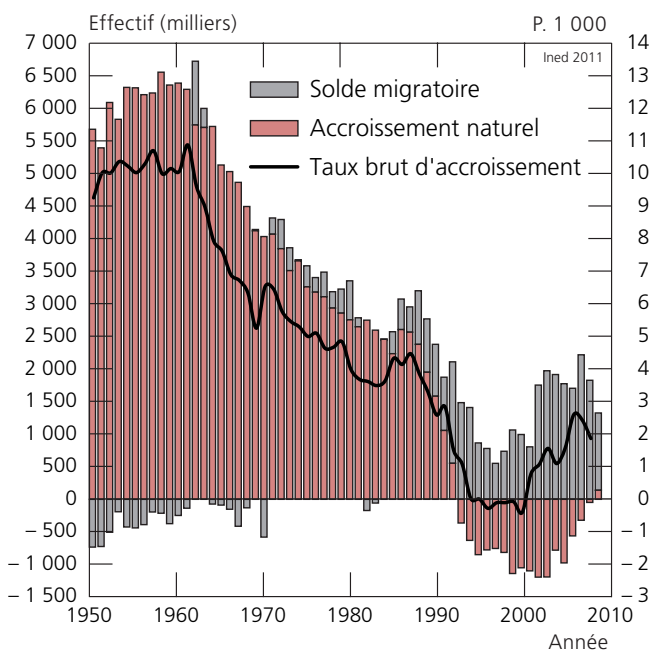

C. Évolution du taux brut d'accroissement naturel de la population dans les pays européens

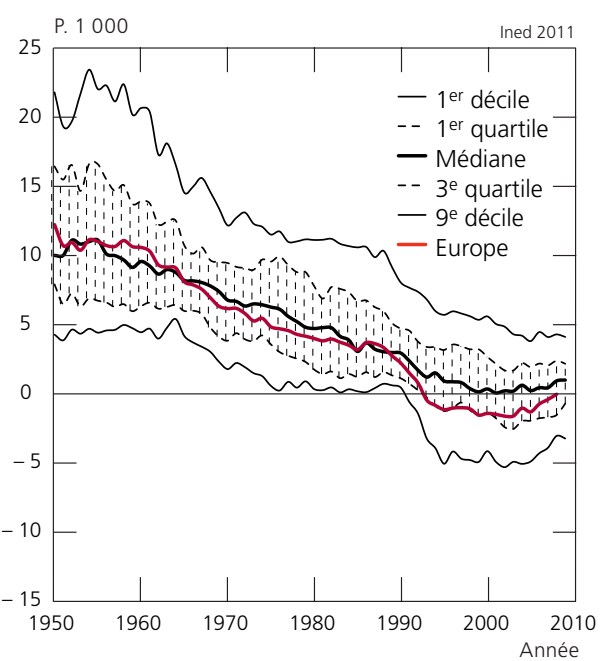

B. Évolution du taux brut d'accroissement de la population dans les pays européens

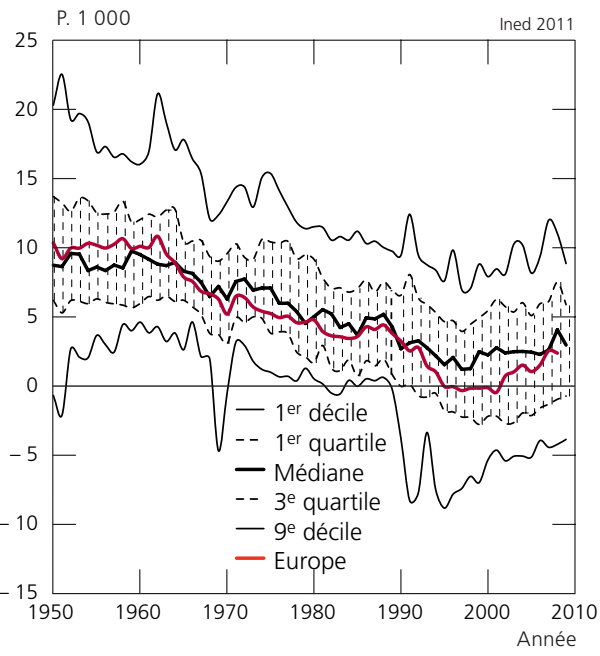

D. Évolution du taux brut d'accroissement migratoire de la population dans les pays européens

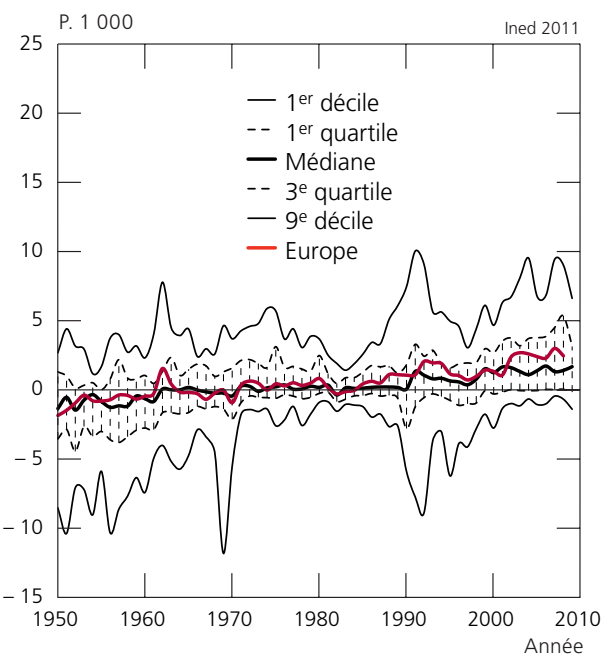

Sources: Base de données des pays développés (Ined) ; base de données Devision du Centre d'études démographiques (Moscou). 
La disparité en hausse des taux de croissance démographique dans les décennies récentes n'est donc pas due au mouvement naturel, mais au solde migratoire (figure 2D). Dans les années 1950, une faible majorité de pays européens et l'Europe dans son ensemble avaient un solde migratoire négatif. De 1962 à 1985, les pays se divisaient en deux parties presque égales : le solde migratoire était positif dans la première et négatif dans la seconde ; l'accroissement migratoire pour l'ensemble de l'Europe oscillait autour de zéro. Il est devenu progressivement positif depuis le milieu des années 1980 ; durant la dernière décennie le solde migratoire a été positif dans deux pays sur trois. En même temps que le solde médian cessait d'être négatif, la dispersion des pays autour de la médiane diminuait (interquartile de quatre points) et devenait très faible au cours des années 1980 (deux points). Depuis lors, la médiane a continué à s'accroître mais la dispersion a sensiblement augmenté au fil du temps, revenant à quatre points dans les années 2000. Sur ce mouvement général se sont greffés des à-coups exceptionnels quand des émigrations massives ont affecté un petit nombre de pays alors que, symétriquement, d'autres pays recevaient un nombre brutalement accru d'immigrants. C'est par exemple le cas des départs d'Albanais vers l'Italie et la Grèce au début des années 1990, perceptibles dans les creux et les pics des premier et dernier déciles.

$\mathrm{Au}$ total, les trois dernières décennies de croissance démographique très faible, voire négative, se situent dans la prolongation d'un mouvement général de ralentissement de la croissance entamé dès la fin des années 1950, au cours duquel l'excédent naturel des naissances sur les décès n'avait cessé de reculer, mais où la contribution du solde migratoire avait compensé partiellement cette évolution. Ces mouvements, qui s'étaient accompagnés d'une convergence des pays européens dans un premier temps, ont cédé la place soit à une stabilisation et un maintien des écarts, soit à un nouveau développement des disparités. L'idée d'une convergence européenne est de ce fait remise en question.

\section{Croissance démographique des pays européens de 1980 à 2009}

$\mathrm{Au} 1^{\mathrm{er}}$ janvier 1980, l'Europe comptait 692,5 millions d'habitants ; au $1^{\text {er }}$ janvier 2009 la population avait augmenté de plus de 40 millions (soit $6 \%$ ) pour atteindre 733,4 millions ${ }^{(7)}$. Dans 22 pays, l'accroissement de la population a été supérieur à $10 \%$. Parmi les pays les plus peuplés, les croissances fortes sont en Espagne (23,1 \%), en France (16,3\%), et au Royaume-Uni (9,5\%) (tableau annexe A.1). La population de Pologne a augmenté de plus de $7 \%$, celle d'Italie de plus de $6 \%$, celle de l'Allemagne de 4,9\%, et celle de Russie de $2,7 \%$. Au total, pour cette période de trente ans, la variation moyenne de

(7) Puisqu'au moment de la rédaction de cet article, les données sur l'effectif de la population au $1^{\text {er }}$ janvier 2010 n'étaient pas disponibles pour l'Albanie, Andorre, la Bosnie-Herzégovine, la Croatie, la Macédoine (ARYM), le Monténégro, la Serbie et l'Ukraine, on ne peut dresser le bilan démographique pour la population de l'Europe que pour la période du $1^{\text {er }}$ janvier 1980 au $1^{\text {er }}$ janvier 2009. Le bilan de population des pays sera dressé soit pour 30 ans (jusqu'au $1^{\text {er }}$ janvier 2010), soit pour 29 ans en fonction de la disponibilité des données. 
l'effectif de population des pays (moyenne arithmétique des variations dans les divers pays ou «moyenne politique ») a été de $15 \%$. Toutefois, dans les douze pays situés sur une ceinture allant de l'Estonie aux Balkans, l'effectif a diminué de $8 \%$ en moyenne (figure 3). Les plus importantes pertes sont affichées par la Bulgarie (- $14 \%)$, la Moldavie et la Lettonie (- $10 \%)$.

Figure. 3. Croissance de la population

des pays européens de 1980 à 2010

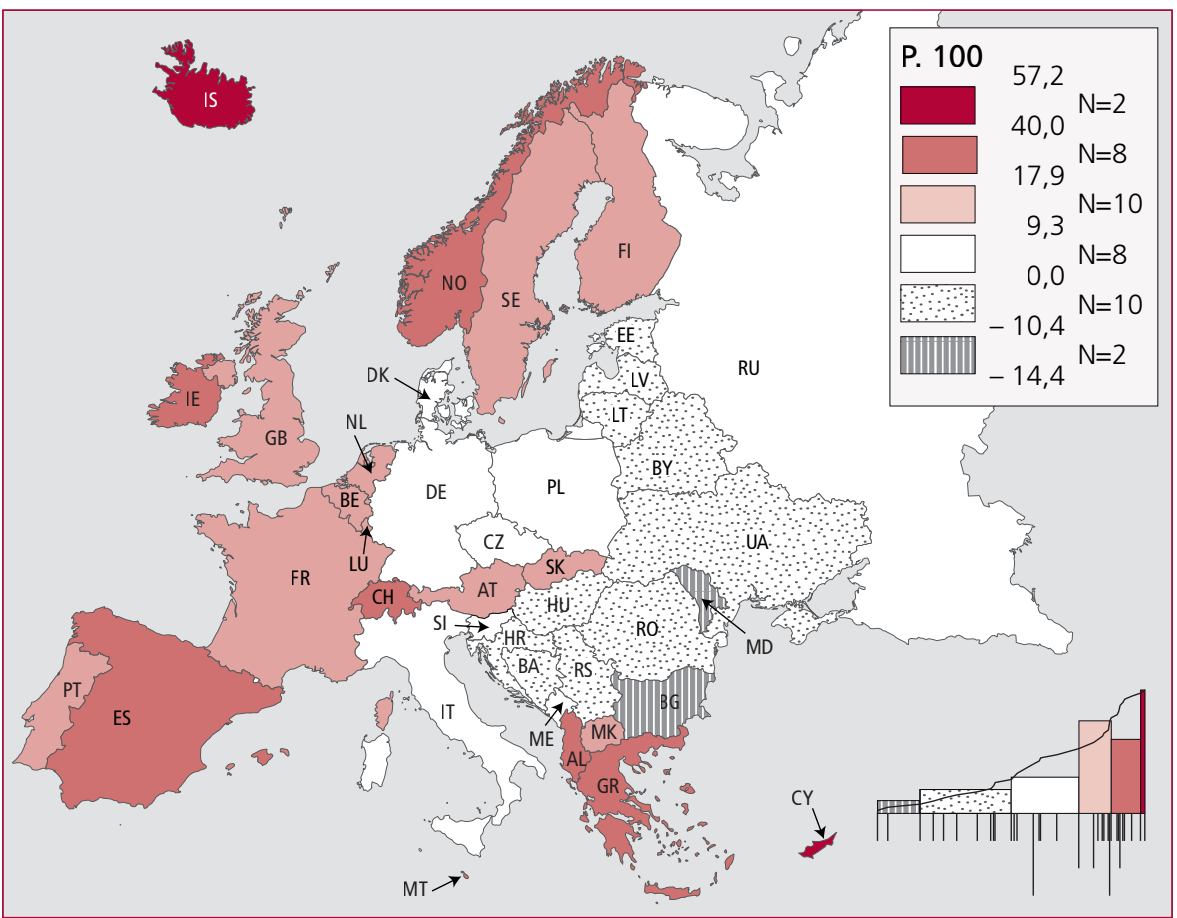

Note : Les effectifs de chaque classe sont aussi proches que possible de la distribution $[2 ; 8 ; 10 ; 10 ; 8 ; 2]$ (voir annexe p. 101). Les histogrammes et la courbe cumulée à droite de la carte présentent la répartition des valeurs. Les surfaces des rectangles de l'histogramme supérieur sont proportionnelles aux nombres de pays dans chaque classe. Dans I'histogramme inférieur, chaque bâton représente la position d'un pays. Sources: Base de données des pays développés (Ined); base de données Devision du Centre d'études démographiques (Moscou).

Des trois décennies, la plus favorable à la croissance démographique a été la première (1980-1990) ; la population augmentait alors dans presque tous les pays à l'exception de la Hongrie (-3\%), la Bulgarie (-0,9\%) et la Macédoine (0 \%). La croissance était la plus rapide en Europe du Sud et de l'Est, la plus faible dans le Nord et l'Ouest.

Durant la décennie suivante, marquée par de profondes transformations sociales dans l'Est de l'Europe, la croissance de l'ensemble de la population européenne s'est quasiment arrêtée. De 1990 à 2000, l'augmentation n'a été que de 4,5 millions de personnes, soit 0,6\%, l'effectif total atteignant 725 mil- 
lions. La population a diminué de $6 \%$ en moyenne dans 17 pays qui abritaient près de 300 millions d'habitants (40\% de la population européenne). Les pertes les plus sensibles ont eu lieu dans les pays ayant connu des conflits armés, notamment la Moldavie et la Bosnie-Herzégovine (-16\%) et deux pays Baltes, l'Estonie et la Lettonie (respectivement - 12\% et - 10 \%). De fait, toute l'Europe orientale est une zone de dépopulation, à l'exception de la Pologne, la Slovaquie et surtout la Macédoine (+ $8 \%$ ).

Entre 2000 et 2009, la République tchèque, la Bosnie-Herzégovine et la Slovénie ont cessé de se dépeupler, mais l'Allemagne et la Pologne ont commencé. La population de l'Europe a augmenté de 1,2\% et le nombre de pays dont la population diminue a été réduit à 14 , mais ces derniers regroupent 378 millions d'habitants, soit plus de la moitié de la population de l'Europe.

\section{Les composantes de la croissance depuis 1980}

\section{Mouvement naturel : bilan des naissances et des décès}

Sur l'ensemble de la période, le bilan des naissances et des décès est positif dans 32 pays européens ${ }^{(8)}$. Pour l'ensemble de la population européenne l'excédent naturel s'élève à $2,3 \%$, et sa valeur moyenne " politique » est égale à $8,2 \%$. Les décès ont été plus nombreux que les naissances dans 8 pays : l'Allemagne et 7 pays d'Europe du Centre et de l'Est, parmi lesquels 5 ont vu diminuer leur population (Hongrie, Ukraine, Lettonie, Bulgarie et Estonie) et 3 ont néanmoins connu une augmentation de leur population entre 1980 et 2010 (Russie, Allemagne et République tchèque) (figure 4).

Les trois décennies qui composent notre période d'étude sont assez contrastées (tableau annexe A.2). Durant la première, " avant la crise », la situation est généralement la plus favorable. Grâce à l'excédent naturel, la population de l'Europe augmente de 3,6 \%, l'accroissement naturel moyen « politique » s'établissant à 5,2 \%. Le bilan des naissances et des décès n'est négatif que dans deux pays, la Hongrie et l'Allemagne (dans ses frontières actuelles).

Durant la décennie 1990-2000, la cartographie de l'accroissement naturel a considérablement changé. L'Europe se divise en deux : 27 pays avec une population totale de 331 millions conservent une croissance naturelle positive, mais 13 pays peuplés de 404 millions d'habitants ont un solde négatif. Une zone de décroissance naturelle s'étend sur presque tous les pays anciennement socialistes (exceptées la Moldavie, la Pologne et la Lituanie); s'y ajoutent l'Allemagne, et au Sud de l'Europe, l'Italie depuis 1993. Trois des États les plus peuplés d'Europe (Russie, Allemagne, Italie) enregistrent donc plus de décès que de naissances.

Dans la décennie suivante, la décroissance naturelle gagne la Lituanie, la Moldavie et la Serbie, tandis qu'elle s'accentue dans tous les pays déjà touchés (sauf en Estonie et République tchèque). Ailleurs, la croissance naturelle se

(8) Rappelons que les données pour Andorre, le Liechtenstein, Saint-Marin et Monaco ne sont pas utilisées dans l'analyse des composantes de la croissance démographique au niveau des pays. 
réduit (sauf en France et au Luxembourg). Laccroissement naturel moyen de la population de l'Europe fléchit encore de - 0,3\% à - $1 \%$.

L'Europe est progressivement devenue une zone où se mèlent une décroissance naturelle assez forte dans 15 pays ( $55 \%$ de la population européenne), et un accroissement naturel très modéré dans 19 pays qui abritent un tiers de sa population. Au fil du temps, l'intensité de la décroissance s'amplifie et celle de l'accroissement s'affaiblit.

Figure 4. Croissance naturelle de la population des pays européens de 1980 à 2010

Solde naturel relatif du 01/01/1980 au 01/01/2010

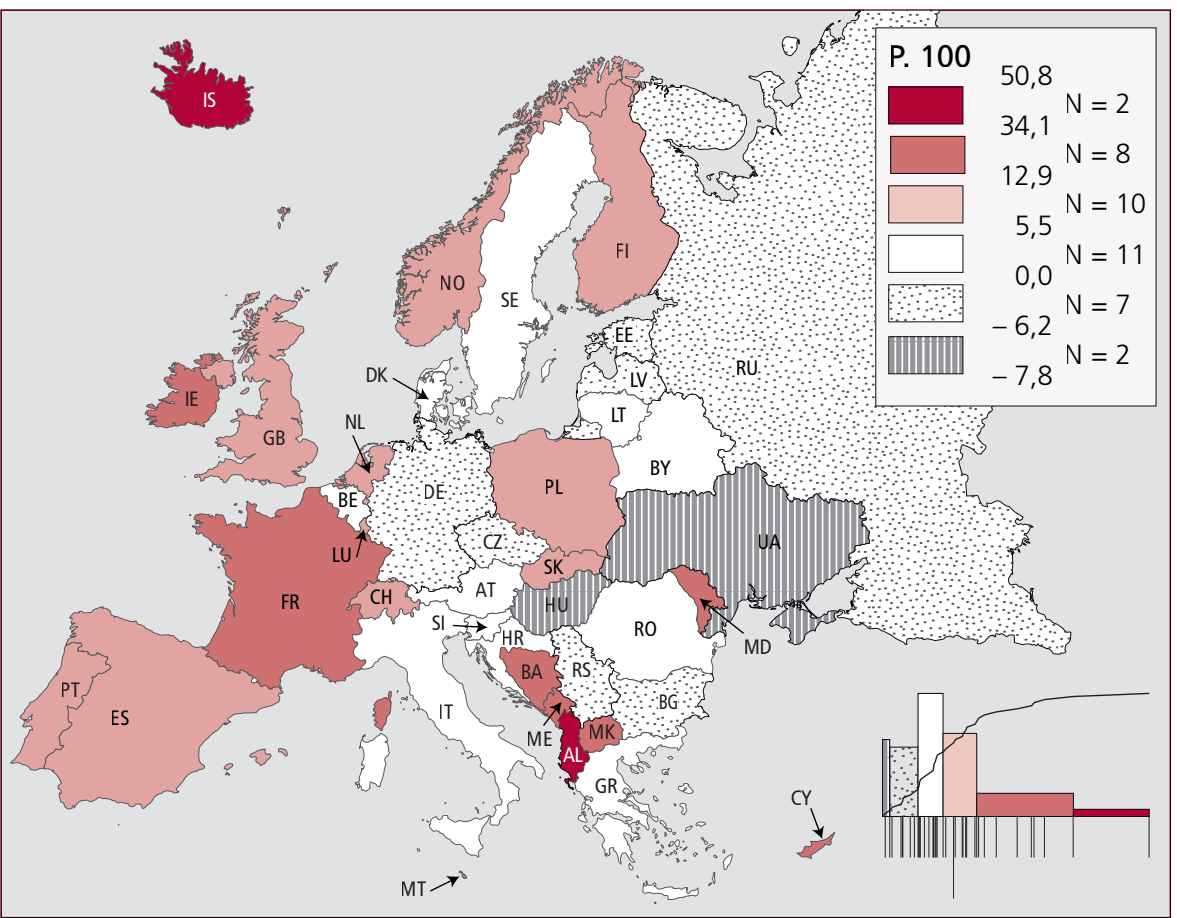

Note: Pour l'Albanie, la Bosnie-Herzégovine, la Macédoine, le Monténégro et l'Ukraine, la variation est estimée sur la période 1980-2009.

Sources: Base de données des pays développés (Ined) ; base de données Devision du Centre d'études démographiques (Moscou)

\section{Composante migratoire du mouvement de population}

Pendant les trois dernières décennies, la migration a fait augmenter la population européenne de 26,5 millions, soit 3,8 \% entre 1980 et 2009, mais ce bilan positif dissimule une grande diversité de situations (tableau annexe A.3). L'accroissement migratoire a été positif dans 24 pays, et en particulier en Espagne et en Suisse (15\%), en Grèce (13\%), en Allemagne, en Norvège, en 
Suisse et en Autriche (8 \% à $9 \%)^{(9)}$ (figure 5). En revanche, en Europe de l'Est et du Centre, la migration a réduit la population en moyenne de près de $11 \%$ dans 16 pays anciennement socialistes ; seules la Russie, la Hongrie, la République tchèque et la Slovénie ont été épargnées. Le flux migratoire le plus important a touché les pays les plus pauvres : l'Albanie a perdu $30 \%$ de sa population, la Moldavie 24\%, la Bosnie-Herzégovine $21 \%$, la Macédoine $16 \%$, le Monténégro $14 \%$. Ainsi, là où le solde migratoire est négatif, la croissance démographique l'est aussi, à l'exception de la Pologne et de la Slovaquie dont la population s'est accrue grâce à l'excédent naturel.

Figure 5. Croissance migratoire de la population des pays européens de 1980 à 2010

Solde migratoire relatif du 01/01/1980 au 01/01/2010

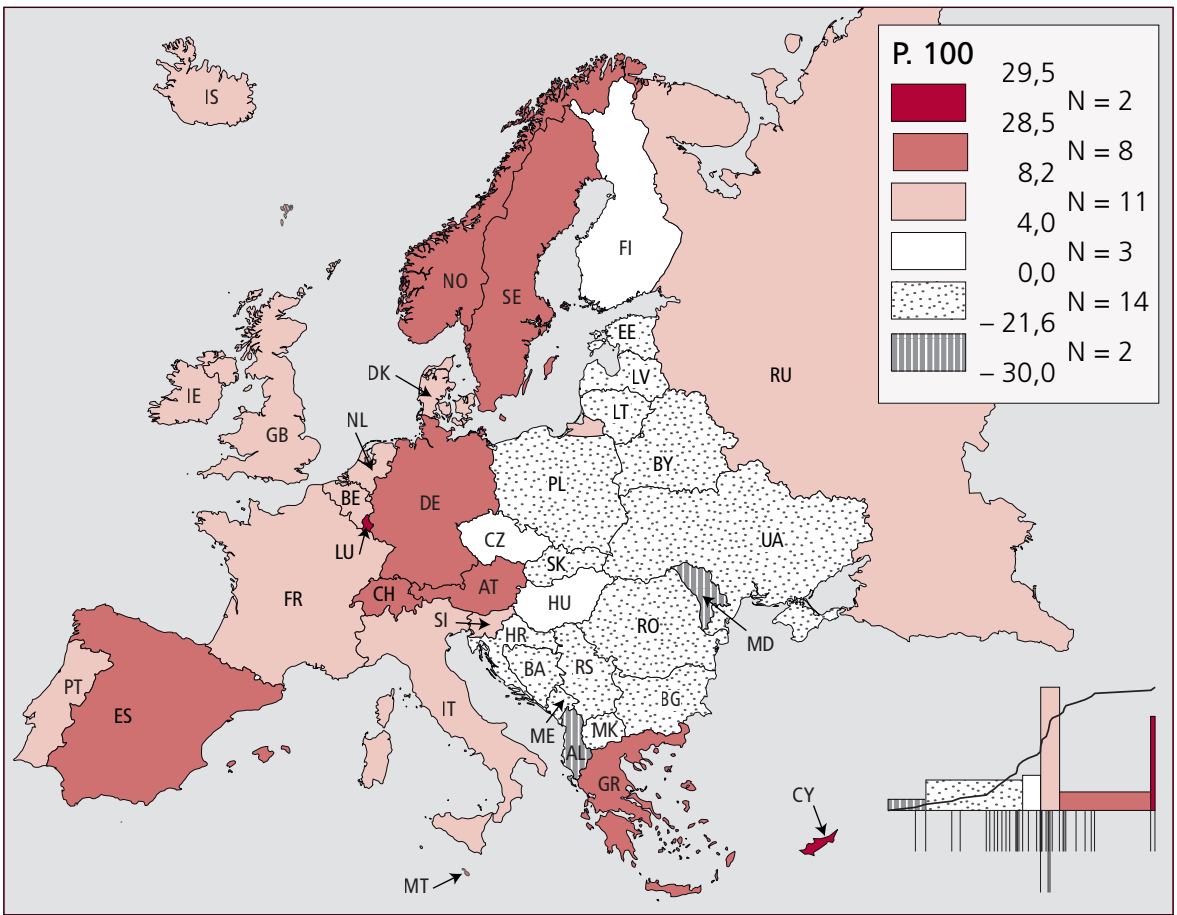

Note: Pour l'Albanie, la Bosnie-Herzégovine, la Macédoine, le Monténégro et l'Ukraine, la variation est estimée sur la période 1980-2009.

Sources: Base de données des pays développés (Ined) ; base de données Devision du Centre d'études démographiques (Moscou).

Entre 1980 et 1990, le solde migratoire a été négatif dans presque tous les pays socialistes du Sud-Est et du Centre de l'Europe, sauf en Serbie et en République tchèque. À l'Ouest, le solde migratoire a été négatif en Irlande, et

(9) Laccroissement migratoire a été le plus fort dans deux petits pays, Chypre et le Luxembourg (près de $30 \%$ ). 
au Sud, au Portugal, en Espagne et en Italie. En revanche, un accroissement migratoire important a eu lieu en Suède, aux Pays-Bas, en Allemagne, en Suisse et en Grèce.

Au cours de la décennie 1990-2000, l'image de la migration en Europe s'est considérablement transformée. D’un côté, l'accroissement migratoire s'amplifie : il a été multiplié par 2 à 2,5 en Grèce, Allemagne, Russie, Suède, Suisse et au Danemark, et il est devenu positif dans tous les pays d'Europe occidentale. De l'autre, l'envergure de la décroissance migratoire reflète l'ampleur des bouleversements politiques des années 1990. L'Albanie a perdu plus de $21 \%$ de sa population, la Moldavie et la Bosnie-Herzégovine $19 \%$. Trois pays très peuplés comme la Roumanie, la Pologne et l'Ukraine ont perdu respectivement 616000,535000 et 298000 habitants. Si l'exode de la population des Balkans et de Moldavie a été provoqué par des conflits militaires, l'émigration massive des pays Baltes et d'Ukraine est la conséquence de la dissolution de l'URSS, qui a entraîné le départ de la population de nationalité russe et russophone en majeure partie vers la Russie. À la fin de la décennie, quand sa situation économique s'est améliorée, la Russie est devenue un pôle d'attraction pour les migrations de travail en provenance des pays voisins. Malgré ces exodes massifs, l'immigration a augmenté la population de l'Europe de presque 6,7 millions en dix ans, soit de 9,2\%o.

Entre 2000 et 2010, les soldes migratoires négatifs touchent moins de pays (9 au lieu de 17) et avec moins de force. Ces pays sont toujours groupés dans la moitié orientale de l'Europe et dans les Balkans, sauf en Bosnie-Herzégovine et en Serbie qui bénéficient probablement du retour de réfugiés. L'Albanie reste le pays de plus forte décroissance migratoire, mais celle-ci n'est plus que de 3,5\%. Au centre de l'Europe, le solde migratoire est devenu positif en Slovaquie, en Slovénie et en Estonie, vraisemblablement à cause de l'amélioration de la situation économique dans ces pays ayant récemment adhéré à l'Union européenne.

Dans les pays à solde positif, celui-ci dépasse 10 \% en Espagne, qui rejoint à ce niveau Chypre et le Luxembourg. Des pays, dont le solde migratoire était traditionnellement négatif, sont désormais parmi les leaders de l'accroissement : outre l'Espagne, ce sont l'Italie et l'Irlande (+ 8,4\%). Par contre, en Allemagne l'accroissement migratoire s'est réduit à 1,1\%.

Dans son ensemble la population européenne a augmenté de 16,7 millions d'habitants (soit 2,6 \%) grâce à la migration durant cette dernière décennie.

\section{La diversité des schémas de fécondité en Europe}

\section{1. Évolution générale de 1960 à 2008}

L'Europe est aujourd'hui relativement homogène en matière de fécondité : les valeurs annuelles les plus récentes ne dépassent plus qu'exceptionnellement le seuil symbolique de deux naissances par femme (tableau annexe A.4). Le 
recul de la fécondité dans les pays où elle était encore élevée est le facteur essentiel d'une légère homogénéisation. Dans les quelques pays où la fécondité est la plus forte (premier décile de la distribution), celle-ci dépassait 3,9 naissances par femme en 1960, 2,4 en 1980 et 1,9 en 2008; soit un recul de 2 points et de plus de $50 \%$. Dans le même temps, le dernier décile (pays de plus basse fécondité) est passé de 2,1 à 1,6 puis 1,3 ; soit un recul de 0,8 point et de presque $40 \%$ (figure 6).

Figure 6. Répartition des pays selon la valeur de l'indicateur conjoncturel de fécondité depuis 1960

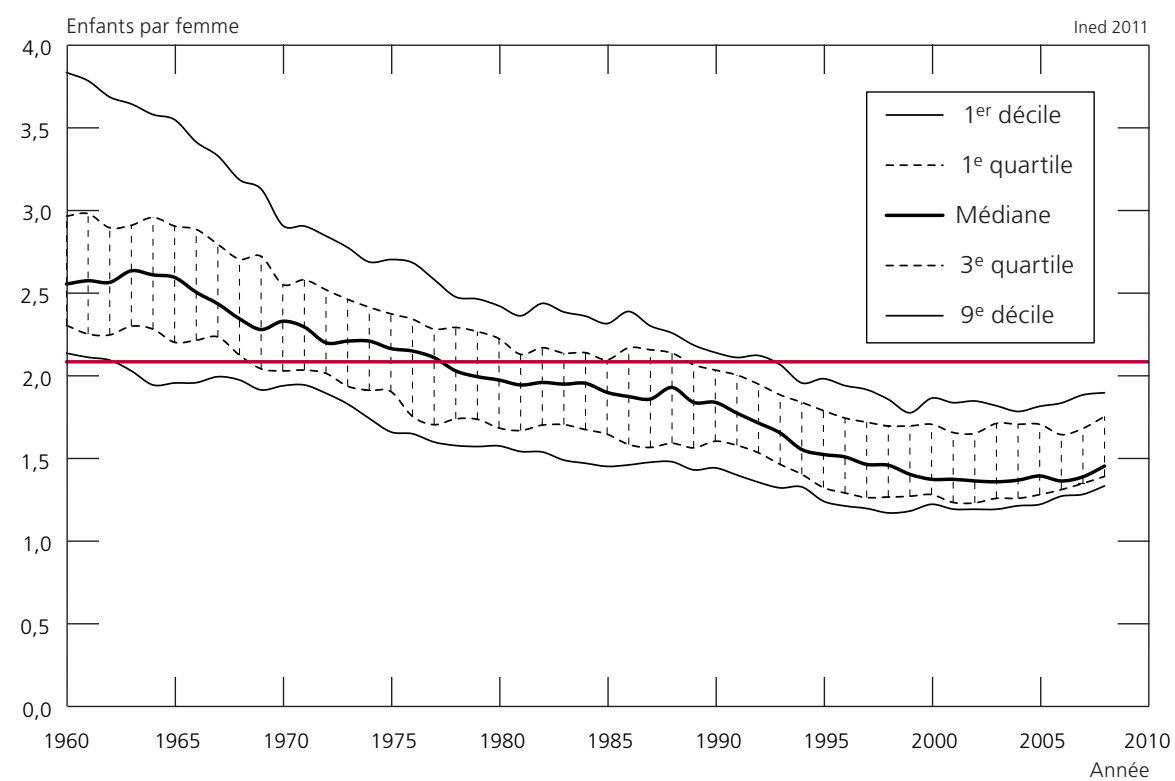

Sources : Base de données des pays développés (Ined) ; base de données Devision du Centre d'études démographiques (Moscou).

Dès 1969, l'indicateur conjoncturel de fécondité (ICF) est descendu sous le seuil de deux enfants par femme en Finlande et en Suède. Les autres pays du Nord et de l'Ouest les ont vite suivies et en 1975, l'ICF était inférieur à 2 dans presque tous les pays de cette région. Dans les pays méditerranéens, c'est un peu plus tard, au début des années 1980, que la fécondité est descendue au-dessous de ce seuil (figure 7A). Au milieu des années 1980, elle devient étonnamment faible dans certains pays très peuplés. Vers 1995, elle y chute en deçà de 1,2 enfant par femme.

La fécondité était devenue inférieure à deux enfants par femme encore plus tôt dans plusieurs pays d'Europe centrale et orientale (1961 en Hongrie). Toutefois, la fécondité s'était ensuite redressée grâce à la mise en œuvre ou au renforcement des politiques d'aides aux familles dans la plupart des pays socialistes. Après une augmentation durant 4-5 ans, les indicateurs de fécondité 
ont commencé à diminuer rapidement. Au cours de la crise du système socialiste à la fin des années 1980 et au début des années 1990, la fécondité s'est écroulée partout, une chute poursuivie durant toute la décennie de transition. L'ICF est devenu inférieur à 1,2 dans certains pays. La diminution la plus spectaculaire a eu lieu en Albanie : encore supérieur à 3 enfants par femme en 1990, l'indicateur est inférieur à 1,4 depuis 2007 (tableau annexe A.4).

Figure 7. Évolution de l'indicateur conjoncturel de fécondité et de l'âge moyen à la maternité dans les régions d'Europe depuis 1960

A. Indicateur conjoncturel de fécondité

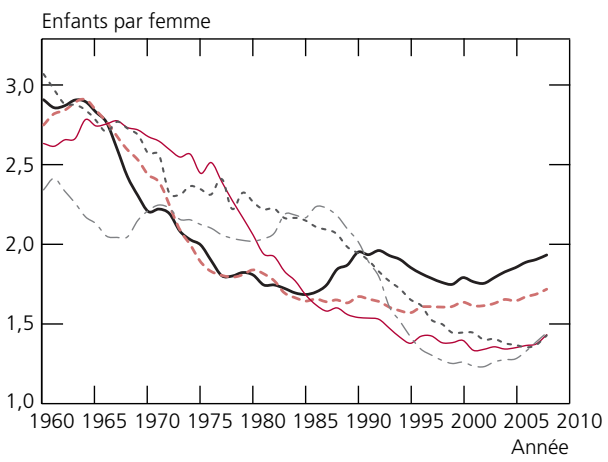

B. Âge moyen à la maternité

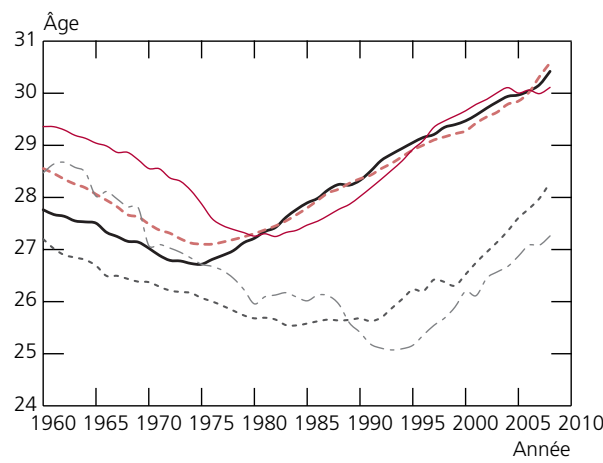

Sources: Base de données des pays développés (Ined) ; base de données Devision du Centre d'études démographiques (Moscou).

Cette baisse très générale de l'ICF pourrait cependant être en partie passagère, provoquée par des changements dans le calendrier de fécondité des générations successives, et renforcée par les difficultés de la période transitoire dans les pays d'Europe du Sud et de l'Est. En effet, depuis le milieu des années 1970, l'âge moyen à la maternité a notablement augmenté dans les pays du Nord et de l'Ouest de l'Europe. Dix ans plus tard, cette tendance s'est manifestée au Sud et au Centre, et dès le milieu des années 1990, elle a touché aussi les pays d'Europe orientale (figure 7B). Toutes autres conditions égales par ailleurs, l'augmentation de l'âge moyen à la maternité provoque une diminution temporaire des indicateurs annuels de fécondité, puisque les plus jeunes générations n'ont pas encore d'enfants, alors que les générations plus âgées n'en font plus car elles ont déjà le nombre désiré d'enfants. Il se pourrait donc que la fécondité des générations successives se déplace vers des âges plus élevés sans changement du niveau final de la descendance (Frejka et Calot, 2001 ; Lesthaeghe, 2001 ; Avdeev, 2003). L'augmentation légère des indicateurs annuels de fécondité depuis le début du $\mathrm{XXI}^{\mathrm{e}}$ siècle dans de nombreux pays européens va dans ce sens. Au Nord de l'Europe et en France, l'ICF s'est approché du seuil de 2 enfants par femme ; mais au Sud et à l'Est, il reste encore très loin de ce niveau. 
Ces tendances conduisent peu à peu à une homogénéisation de la fécondité des pays européens, et la coupure n'est plus entre l'Ouest et l'Est mais entre le Nord-Ouest, le Sud et l'Est (figure 8). Examinons ces évolutions, en distinguant la fécondité et son niveau des autres aspects des transformations familiales.

Figure 8. Indicateur conjoncturel de fécondité dans les pays d'Europe en 2008 (nombre moyen d'enfants par femme)

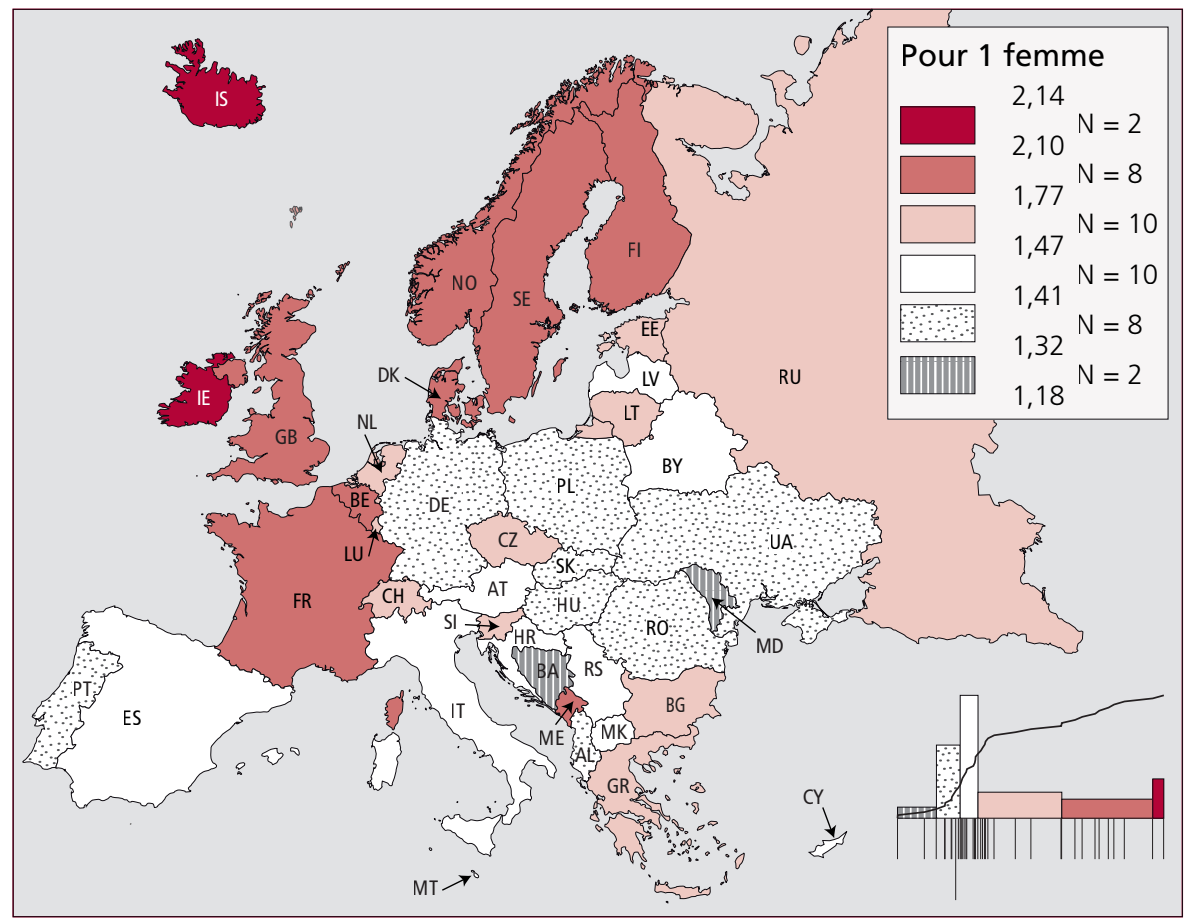

Sources: Base de données des pays développés (Ined) ; base de données Devision du Centre d'études démographiques (Moscou).

\section{Niveau de fécondité et âge à la maternité des années 1970 aux années 2000}

Sur la figure 9 sont associés l'indicateur conjoncturel de fécondité et l'âge moyen à la maternité dans les cinq régions d'Europe, décennie par décennie depuis les années 1970. On visualise ainsi le passage d'une fécondité encore relativement forte à une fécondité basse (de droite à gauche sur le graphique), concomitamment à l'élévation de l'âge des mères à la naissance de leurs enfants (de bas en haut sur le graphique).

On observe donc deux trajectoires pour la fécondité européenne des quarante dernières années. Dans le Nord et l'Ouest du continent, la période a été dominée par l'élévation de l'âge à la maternité d'au moins trois ans entre les années 1970 et les années 2000 ; dans le même temps, le nombre annuel 
d'enfants par femme a peu varié. Ailleurs, au Sud, au Centre comme à l'Est, le fait majeur a été la baisse de la fécondité, bien que celle-ci ait été ralentie, voire stoppée récemment; à l'inverse, l'âge à la maternité est resté longtemps stable, avant d'augmenter brutalement dans les années récentes. La hiérarchie des niveaux de fécondité s'est renversée, les indicateurs étaient plus bas au Nord et à l'Ouest qu'ailleurs dans les années 1970, contrairement à aujourd'hui. Le retard de l'âge à la maternité est devenu un trait commun à toutes les régions d'Europe depuis cinq à dix ans, laissant subsister un écart de l'âge moyen entre la moitié ouest et la moitié est du continent, dont on sait qu'il s'enracine dans un lointain passé.

Figure 9. Indicateur conjoncturel et âge moyen à la maternité par région de 1975 à 2005

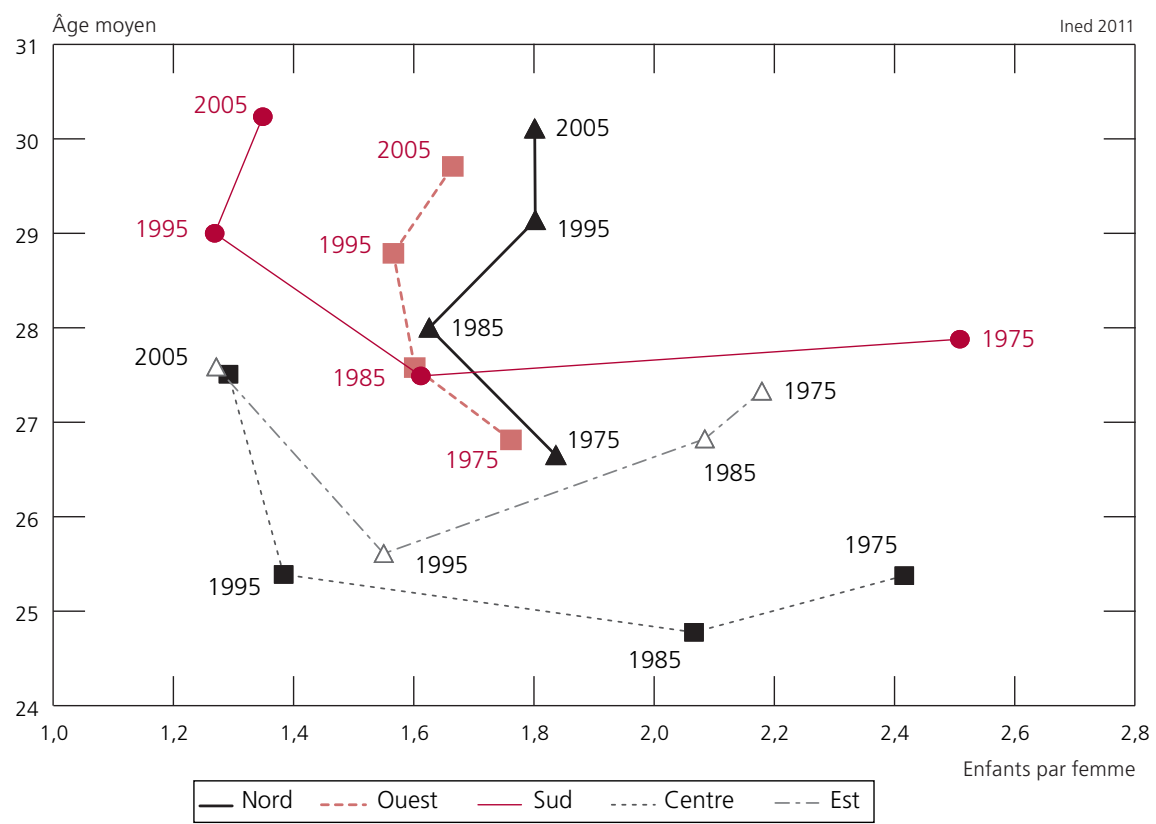

Source: Calculs de l'auteur à partir de la base de données d'Eurostat.

\section{Fécondité par âge selon les générations}

L'importance prise par les changements du calendrier de maternité incite à examiner la fécondité aux différents âges. Le schéma général est celui d'un recul de la fécondité aux jeunes âges « compensé » partiellement ou totalement par une hausse aux âges plus avancés. On glisse du constat vers son interprétation en termes de déplacement des âges de maternité, d'attente, de retard. De telles notions n'ont de sens que si la perspective est longitudinale - et non transversalele même groupe de femmes étant suivi au fil du temps, afin de voir si les grossesses que ces femmes n'ont pas eues aux jeunes âges ont lieu à des âges 
plus avancés. Nous ne conservons qu'un petit échantillon de pays illustratifs des mécanismes en action dans chaque région : la Suède au Nord, les Pays-Bas à l'Ouest, l'Italie au Sud, la Bulgarie au Centre ${ }^{(10)}$ (figure 10).

Dans les pays occidentaux (Suède, Pays-Bas, Italie), les courbes des taux de fécondité par âge se sont déplacées progressivement mais très nettement des jeunes âges vers les âges avancés. L'âge modal, auquel la fécondité passe par un sommet, a augmenté très sensiblement : en Suède et aux Pays-Bas, il est passé de 26-27 ans à 31 ; en Italie, de 24 à 30 ans. Ce sont des changements considérables. Toutefois, en Italie, le relèvement de la fécondité à partir de 29 ans n'a pas compensé son abaissement en deçà, et il y a donc baisse de la descendance finale : en 15 générations, elle est passée de 1,7 à 1,4 naissance par femme. En Suède et aux Pays-Bas, la compensation a été plus complète, la baisse des descendances finales moindre.

Figure 10. Taux de fécondité par âge dans les générations 1960 à 1975 en Suède, aux Pays-Bas, en Italie et en Bulgarie.
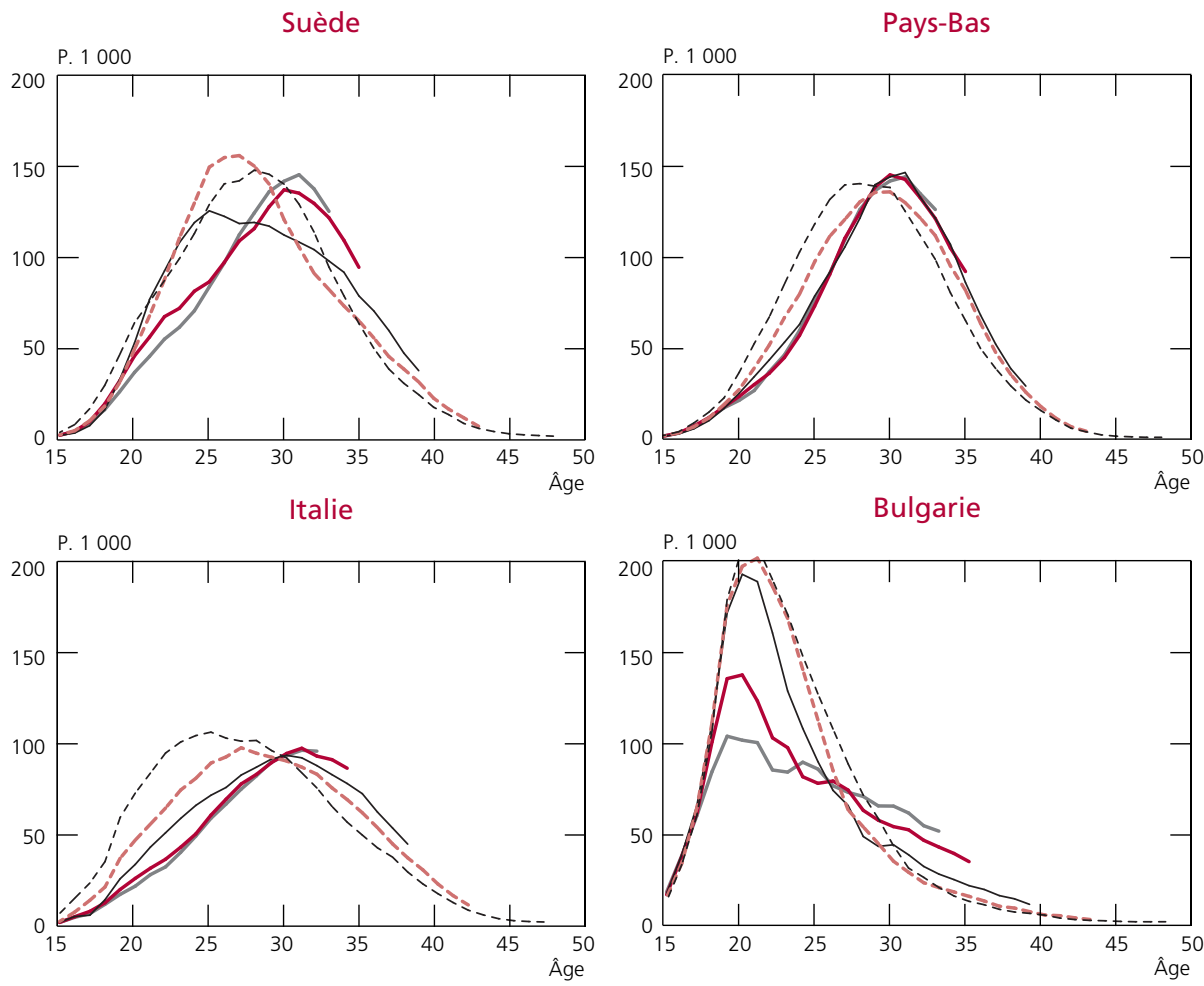

\begin{tabular}{|c|c|}
\hline _. _ 1960 & - -1965 \\
\hline
\end{tabular}

Source : Calculs de l'auteur à partir de la base de données d'Eurostat.

(10) La Lituanie à l'Est a un profil très voisin de celui de la Bulgarie. 
Dans les pays orientaux, la fécondité est nettement plus précoce que dans la moitié occidentale du continent. C'est particulièrement marqué en Bulgarie, où les femmes nées en 1960 avaient un maximum d'enfants dès 20-21 ans. Les changements du calendrier de la fécondité au fil des générations se sont traduits, non par un déplacement du mode de distribution mais par un très fort abaissement de son niveau. Comme à l'Ouest cependant, la fécondité a augmenté au-delà de 30 ans, compensant ainsi partiellement la diminution antérieure. Partiellement seulement puisque les descendances finales ont reculé, de 1,9 à 1,5 naissance par femme, alors que l'âge moyen à la maternité augmentait de 2 ans.

\section{4. Évolution de la fécondité au fil des années et des générations}

On sait qu'un retard de fécondité d'une génération à l'autre, puisque les femmes remettent à plus tard la naissance de leurs enfants, tend à déprimer les indicateurs conjoncturels qui deviennent inférieurs aux descendances finales, bilan effectif des vies fécondes. On s'attend donc à ce que les indicateurs annuels depuis 1970 en Europe de l'Ouest aient sous-estimé au long de ces décennies la fécondité effective des générations féminines, puisque l'augmentation de l'âge à la maternité s'est maintenue sur plus de trente ans. À l'est du continent, où les changements d'âge ont été plus récents, le décalage entre mesures transversales et longitudinales devrait être visible depuis moins longtemps (figure 11).

Figure 11. Indicateur conjoncturel de fécondité et descendance finale (décalée de 28 ans) en Suède, aux Pays-Bas, en Italie et en Bulgarie
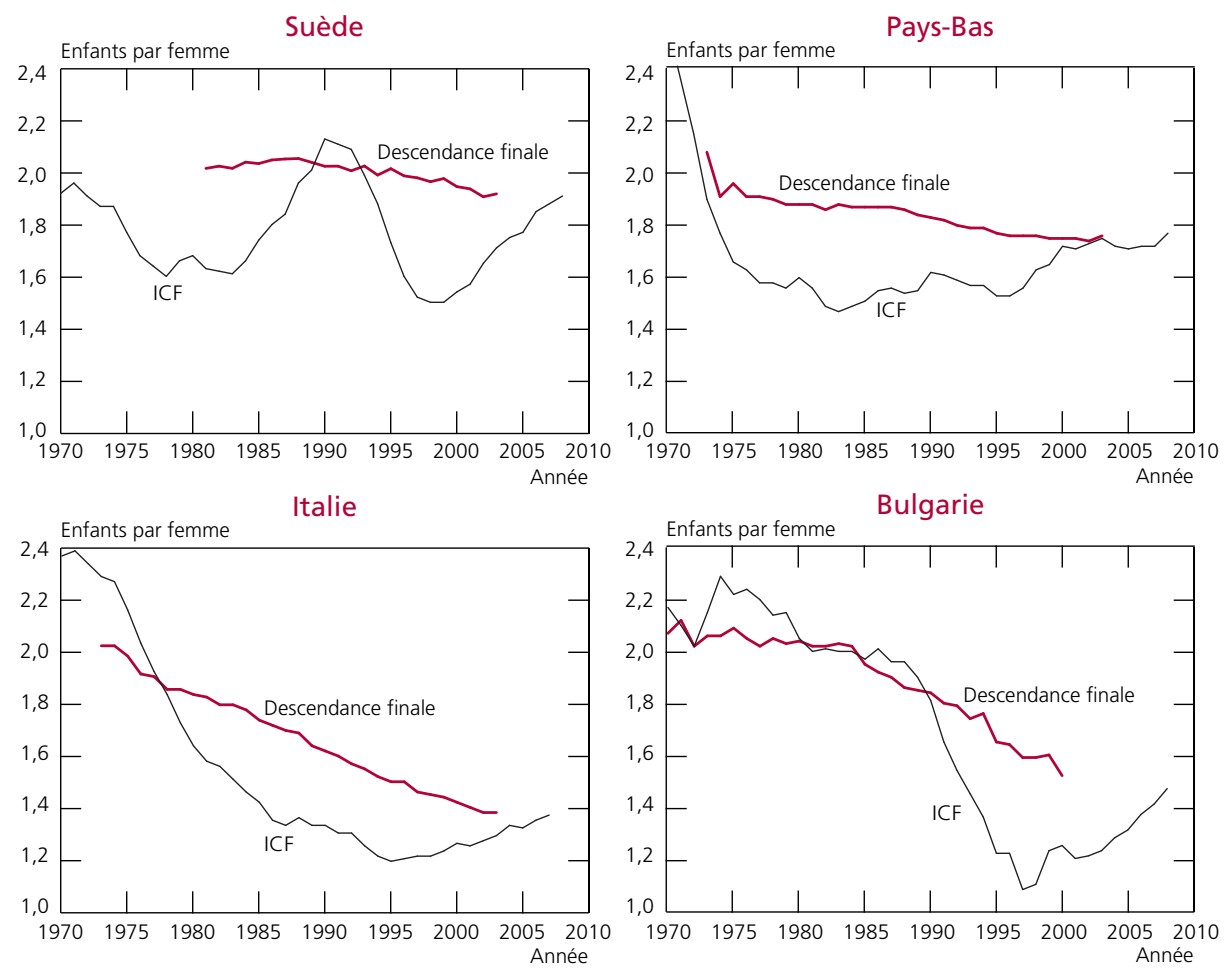

Source : Calculs de l'auteur à partir de la base de données d’Eurostat. 
La réalité est conforme aux attentes, mais il existe des nuances non négligeables entre les pays. En Suède, la descendance des générations s'est établie autour de deux naissances par femme dans un lent mouvement de recul (de 2,05 à 1,91 des générations 1960 à 1975) ; les indicateurs conjoncturels étaient descendus à 1,6 naissance par femme autour de 1980, puis 1,5 vers 2000, ces deux épisodes étant séparés par une phase de reprise de 1986 à 1993 facilitée par des politiques favorisant la naissance des enfants à intervalles rapprochés ${ }^{(11)}$. L'augmentation en cours aujourd'hui ramène les indicateurs conjoncturels vers un niveau qui semble s'accorder à celui des descendances de génération (1,9 naissance par femme). Le schéma général n'a pas été très différent aux Pays-Bas et en Italie - hormis la reprise « politique »- mais le recul tendanciel des descendances finales a été plus marqué, surtout en Italie; les mesures transversales et longitudinales se rapprochent fortement en fin de période, au niveau de 1,75 naissance par femme aux Pays-Bas et seulement 1,4 en Italie. Si, dans ces trois pays, les indicateurs récents sont à la hausse et convergent vers les descendances finales, c'est dans un mouvement de reprise franche vers un niveau relativement élevé en Suède, une hausse modérée vers des valeurs moyennes aux Pays-Bas et un relèvement très modeste vers des fécondités encore très basses en Italie.

À l'est de l'Europe, la conjoncture des dernières décennies a été très différente, marquée par le brutal changement politique du début des années 1990. Les indicateurs transversaux ont chuté fortement peu après, et ils tendent à se redresser récemment (Bulgarie, figure 11). La descendance des générations a baissé tendanciellement; les indicateurs transversaux ont amplifié ce mouvement en atteignant de très bas niveaux un peu avant ou un peu après 2000, les naissances ayant été retardées un temps puis partiellement récupérées. La convergence des descendances et des indicateurs conjoncturels semble se situer vers 1,5 naissance par femme, niveau bien supérieur aux très basses valeurs des indices des années autour de 2000, mais très en recul par rapport aux niveaux des années antérieures à 1990.

La discordance de niveau, voire de tendance, entre les indicateurs annuels de fécondité (ICF) et ceux qui synthétisent l'histoire des générations (descendances finales) est un sujet récurrent de l'analyse des évolutions démographiques en Europe depuis au moins quarante ans. Le mouvement continu et commun à tous les pays vers une maternité plus tardive fait partout des mesures transversales une évaluation par défaut de la fécondité des générations. Dans le cas de la Suède, par exemple, la baisse forte des indicateurs conjoncturels

(11) En Suède, les parents bénéficient d'un congé parental rémunéré après la naissance d'un enfant. Son montant est de $80 \%$ du salaire antérieur à la naissance. Si la naissance d'un deuxième enfant survient à un intervalle suffisamment rapproché, les parents peuvent conserver après la naissance du deuxième le montant de l'allocation précédemment versée, même si entre-temps ils occupent un emploi moins rémunérateur (par exemple à temps partiel). Les Suédois la nomment « prime à la vitesse ». En 1980, cet avantage était acquis quand l'intervalle entre deux naissances était inférieur à 24 mois. En 1986, l'intervalle a été étendu à 30 mois, bénéficiant ainsi à un nombre sensiblement plus important de parents. 
dans les années 1970 a tenu essentiellement au retard de la maternité, pas à une baisse des descendances dans les générations. La nette reprise de la seconde moitié des années 1980, qui a accompagné l'adoption d'une politique familiale favorisant les naissances rapprochées, n'a eu aucune répercussion sur les descendances de génération. La seule conséquence de ces mesures a été un ralentissement de l'augmentation des âges à la maternité (faible augmentation dans les générations 1960-1965), le mouvement de fond étant partiellement contrecarré par un raccourcissement des intervalles entre naissances. Comme c'est souvent le cas, la lecture des indicateurs annuels de fécondité a pu faire prendre pour une augmentation de la taille des familles, une évolution qui n'était qu'une fluctuation du calendrier de constitution de la descendance.

Dans les années récentes, le redressement sensible de la fécondité en Suède et dans divers pays à l'ouest du continent suggère beaucoup moins un relèvement de la descendance des générations qu'une pause dans le retard de la fécondité, qui pourrait annoncer que ce mouvement d'élévation des âges à la maternité atteint ses limites.

\section{Rang de naissance et taille de la famille}

Un contraste fort et durable s'est établi entre des descendances de générations faiblement déclinantes, voire stables, au Nord-Ouest de l'Europe, et des descendances déclinantes et basses ailleurs, en particulier au Sud.

En Suède, par exemple, non seulement la descendance est stabilisée pour les générations féminines récentes (1965-1975), mais la distribution des tailles de famille a peu évolué (tableau 2). La proportion de femmes sans enfant n'a augmenté que faiblement, de $12 \%$ à $14 \%$. Les proportions de celles avec un enfant unique ou deux enfants sont à peu près stables, la première très minoritaire (à peine plus de $15 \%$ ), la seconde proche de la moitié (45\%). Seule a décliné la part des femmes constituant une famille d'au moins trois enfants, et encore le recul a-t-il été modéré (de $29 \%$ à $25 \%$ en dix générations).

En Espagne, par contre, la distribution des tailles de famille a beaucoup changé. La proportion de femmes sans enfant a augmenté fortement en dix générations (passant de 16 \% à $26 \%$ ). Celle des mères d'enfant unique a également progressé, mais de façon nettement plus modérée, passant dans le même temps de $28 \%$ à $30 \%$. L'autre écart sensible avec la Suède a été le fort recul de la proportion de familles de deux enfants (de $44 \%$ à $35 \%$ ), alors que les familles de trois enfants ou plus, déjà plus rares, ont encore perdu trois points (de $12 \%$ à $9 \%$ ).

Pour la génération la plus récente née en 1975, la distribution des familles par taille révèle l'ampleur des écarts entre le Sud et le Nord de l'Europe aujourd'hui. En Suède, ces femmes, qui ont eu leurs enfants pour l'essentiel dans les années 2000, sont une minorité à rester sans enfant (14\%) ou avec un enfant unique (16\%); elles sont une large majorité à avoir au moins deux enfants (45\% en ont deux, $25 \%$ au moins trois). En Espagne, à l'inverse, les 
descendances restreintes sont majoritaires (26\% sans enfant, $30 \%$ avec un seul enfant) et celles d'au moins deux enfants minoritaires (35\% avec deux enfants, $9 \%$ avec trois ou plus). Pour s'être creusés fortement dans les dix dernières générations, les écarts sont particulièrement remarquables pour les femmes sans enfant, situation devenue beaucoup plus fréquente en Espagne qu'en Suède, et pour les femmes avec deux enfants, phénomène nettement plus rare en Espagne.

Tableau 2. Caractéristiques de la fécondité des générations 1965 à 1975 par rang de naissance en Suède et en Espagne

\begin{tabular}{|c|c|c|c|c|c|c|c|c|c|c|c|}
\hline & \multicolumn{11}{|c|}{ Génération } \\
\hline & 1965 & 1966 & 1967 & 1968 & 1969 & 1970 & 1971 & 1972 & 1973 & 1974 & 1975 \\
\hline \multicolumn{12}{|c|}{ Suède } \\
\hline \multicolumn{12}{|c|}{ Proportion de femmes (\%) avec } \\
\hline 0 enfant & 12 & 13 & 12 & 12 & 12 & 13 & 12 & 13 & 13 & 14 & 14 \\
\hline 1 enfant & 15 & 15 & 15 & 15 & 16 & 15 & 15 & 16 & 16 & 16 & 16 \\
\hline 2 enfants & 44 & 43 & 45 & 45 & 45 & 46 & 46 & 46 & 45 & 45 & 45 \\
\hline 3 enfants ou plus & 29 & 28 & 28 & 27 & 27 & 26 & 26 & 26 & 26 & 25 & 25 \\
\hline \multicolumn{12}{|c|}{ Âge moyen à la maternité à la } \\
\hline $1^{1 \text { ère }}$ naissance & 26,8 & 26,9 & 27,0 & 27,2 & 27,3 & 27,5 & 27,8 & 28,0 & 28,2 & 28,4 & 28,5 \\
\hline $2^{\mathrm{e}}$ naissance & 29,4 & 29,5 & 29,6 & 29,7 & 30,0 & 30,2 & 30,4 & 30,6 & 30,8 & 31,0 & 31,1 \\
\hline $3^{\mathrm{e}}$ naissance & 31,6 & 31,7 & 31,9 & 32,0 & 32,2 & 32,4 & 32,6 & 32,8 & 32,9 & 33,1 & 33,1 \\
\hline \multicolumn{12}{|c|}{ Espagne } \\
\hline \multicolumn{12}{|c|}{ Proportion de femmes (\%) avec } \\
\hline 0 enfant & 16 & 18 & 18 & 18 & 21 & 21 & 22 & 23 & 25 & 25 & 26 \\
\hline 1 enfant & 28 & 27 & 28 & 29 & 28 & 29 & 29 & 30 & 30 & 30 & 30 \\
\hline 2 enfants & 44 & 43 & 43 & 43 & 41 & 40 & 39 & 38 & 37 & 35 & 35 \\
\hline 3 enfants ou plus & 12 & 11 & 11 & 11 & 10 & 10 & 10 & 9 & 9 & 9 & 9 \\
\hline \multicolumn{12}{|c|}{ Âge moyen à la maternité à la } \\
\hline $1^{\text {ère }}$ naissance & 27,2 & 27,6 & 27,8 & 28,1 & 28,5 & 28,9 & 29,3 & 29,6 & 29,9 & 30,0 & 30,2 \\
\hline $2^{\mathrm{e}}$ naissance & 30,8 & 31,0 & 31,3 & 31,5 & 31,7 & 32,0 & 32,2 & 32,4 & 32,5 & 32,7 & 32,7 \\
\hline $3^{\mathrm{e}}$ naissance & 32,5 & 32,8 & 33,0 & 33,2 & 33,4 & 33,6 & 33,8 & 33,9 & 33,9 & 34,0 & 34,1 \\
\hline
\end{tabular}

À la divergence des mouvements en matière de taille des descendances, il faut opposer, une fois de plus, les évolutions parallèles des âges aux naissances de divers rangs. En Suède comme en Espagne, la hausse a été forte. En dix générations, les âges moyens ont augmenté en général de 1,5 à 2 ans, quel que soit le rang de naissance. Ceci suggère une certaine stabilité des intervalles entre naissances successives, la première maternité ayant été retardée alors que les autres, quand il y en a, ont suivi à un rythme maintenu. La seule exception concerne les premières naissances en Espagne, repoussées de 3 ans, un mouvement un peu atténué aux rangs suivants. Ceci suggère un raccourcissement de l'intervalle 
entre la première et la deuxième naissance ; mais il est possible aussi que se soit développé un groupe spécifique de femmes ayant eu leur premier enfant à un âge avancé et de ce fait, le seul dans la constitution de leur descendance.

\section{Fécondité et formes familiales}

\section{Nuptialité et naissances hors mariage des années 1970 aux années 2000}

Les quatre décennies entre 1970 et 2010 ont été marquées à la fois par un recul spectaculaire du mariage et un fort développement des naissances hors mariage (tableau annexe A.5). Les deux mouvements sont liés, l'effacement de la nuptialité laissant place à des unions moins formelles dans lesquelles peut s'inscrire la fécondité des couples, même si c'est à des degrés divers selon les époques et selon les pays. Dans l'espace européen, cette relation s'exprime généralement dans une liaison négative entre les indicateurs conjoncturels de nuptialité(12) sur une période et la proportion de naissances hors mariage quelques années plus tard. Plus la nuptialité est basse, plus la part des naissances hors mariage est élevée (figures 12 et 13).

Figure 12. Indicateur conjoncturel de nuptialité (pour 100 femmes) et proportion de naissances hors mariage 5 ans plus tard (\%)

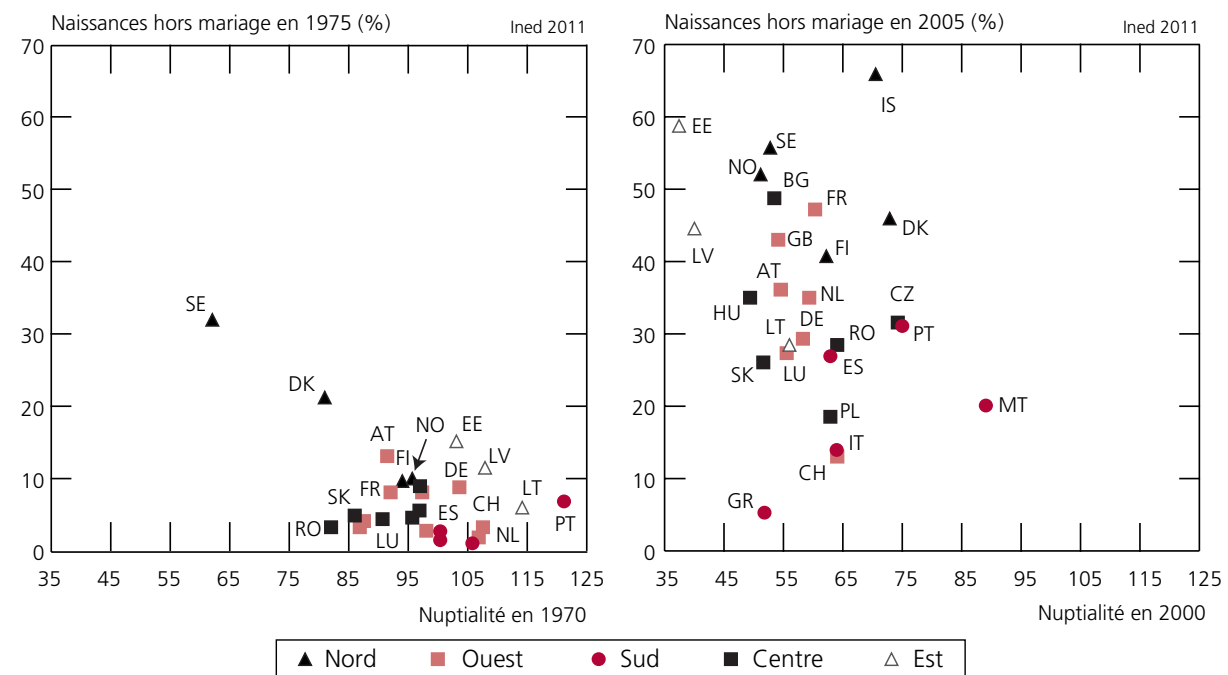

Source : Calculs de l'auteur à partir de la base de données d'Eurostat.

(12) Somme des taux de primo-nuptialité des femmes par âge, de 15 à 49 ans, les taux par âge étant calculés en rapportant les premiers mariages à l'ensemble des femmes du même âge. Comme l'indicateur conjoncturel de fécondité, l'indicateur conjoncturel de nuptialité peut s'écarter sensiblement de la fréquence des premiers mariages avant 50 ans dans les générations : en période de rajeunissement de la nuptialité, son niveau surestime la nuptialité et peut dépasser $100 \%$. 
Figure 13. Nuptialité et naissances hors mariage 5 ans plus tard par région au fil du temps

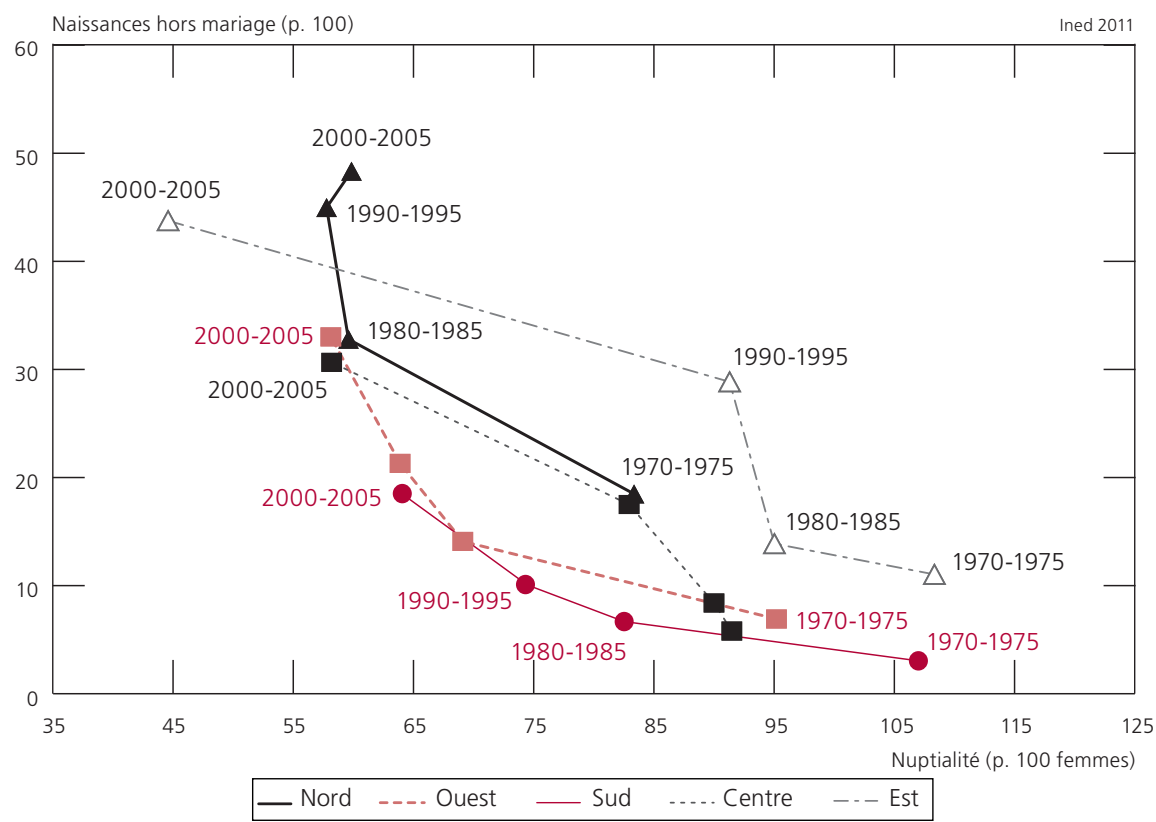

Source: Calculs de l'auteur à partir de la base de données d'Eurostat.

Dans les années 1970, la nuptialité domine encore dans presque tous les pays d'Europe et la fécondité hors mariage est rare. Seuls le Danemark et la Suède voient la nuptialité sensiblement reculer et la part des naissances hors mariage dépasser $20 \%$ ou $30 \%$. Ces pays pionniers commencent à dessiner une relation négative entre nuptialité et fécondité hors mariage. Ailleurs, les indicateurs de nuptialité dépassent souvent $100 \%$, attestant ainsi d'une précocité croissante du mariage au Sud, à l'Ouest et à l'Est du continent (pas au Centre). Sous l'effet de ce phénomène, la dispersion est relativement importante, entre moins de $90 \%$ et plus de $120 \%$; bien davantage que la dispersion des proportions de naissances hors mariage, presque toutes inférieures à $10 \%$.

Dix ans plus tard, dans les années 1980, les comportements observés au Danemark et en Suède s'étaient amplement diffusés dans les pays nordiques et divers pays de l'Ouest. L'Europe du Sud connaît elle aussi une baisse de la nuptialité, mais la part des naissances hors mariage reste très modérée. Quant aux pays du Centre et de l'Est, ils ont les indicateurs de nuptialité désormais les plus élevés d'Europe.

À partir des années 1990, la relation négative entre nuptialité et fécondité hors mariage affecte l'ensemble du continent. En Europe occidentale, nuptialité faible et part élevée des naissances hors mariage au Nord contrastent avec une nuptialité encore relativement forte au Sud, quoiqu'en net recul, et une fécondité 
hors mariage encore faible ; les pays de l'Ouest ont une position intermédiaire. La plupart des pays d'Europe orientale sont encore très différents ; la relation négative va de pair avec un niveau de nuptialité beaucoup plus élevé.

Dans les années 2000, l'ensemble des pays d'Europe partagent un régime de nuptialité basse, les derniers pays d'Europe orientale ayant rejoint le reste du continent. La diversité est surtout due à la fécondité hors mariage, dans une relation inverse beaucoup plus discrète avec la nuptialité. La part des naissances hors mariage reste très élevée partout au Nord (au-dessus de $40 \%$ ), mais aussi en France et au Royaume-Uni à l'Ouest, en Slovénie et en Bulgarie au Centre, en Estonie et en Lettonie à l'Est. Les pays méridionaux présentent tous des proportions inférieures à $30 \%$.

Les évolutions au fil du temps des différentes régions se sont toutes faites dans la même direction vers une nuptialité plus faible et une proportion plus élevée de naissances hors mariage. Le mouvement a été régulier et progressif en Europe de l'Ouest et du Sud, avec un décalage de dix à quinze ans. Il avait été sensiblement plus brutal au Nord, comme il le sera en Europe orientale vingt ans plus tard à partir des années 1990 (figure 13).

\section{Le premier mariage dans les générations féminines}

L'exemple des Pays-Bas rend compte du renversement de tendance qui s'est opéré à l'Ouest de l'Europe dans les années 1970-1980 pour les femmes nées dans les années 1950 (figure 14). La proportion de femmes célibataires ${ }^{(13)}$ à 20-24 ans, qui était passée en vingt générations de $70 \%$ à $45 \%$, est remontée aussi rapidement à plus de $80 \%$ avant de s'acheminer plus lentement vers $90 \%$. Les femmes qui se marient avant 20-24 ans ne représentent plus, dans les générations récentes, qu'une très faible minorité, du fait non seulement de la raréfaction des mariages précoces mais aussi des femmes de plus en plus nombreuses qui ne se marieront sans doute jamais, comme l'atteste l'augmentation de la proportion de célibataires à 45-49 ans. La nuptialité des générations devient plus tardive et plus rare, à l'inverse de la précocité et l'intensité plus grandes qui avaient caractérisé, après-guerre, l'âge d'or du mariage.

Ce phénomène est encore plus visible en Suède, où il avait commencé plus tôt. La progression du célibat à 20-24 ans semble avoir atteint aujourd'hui une limite, autour de $92 \%$. Il en va de même à des âges plus avancés, suggérant que les caractéristiques du mariage rare et tardif pourraient se stabiliser, par exemple avec trois quarts de femmes encore célibataires à 25-29 ans (contre moins d'un quart trente ans plus tôt), et un tiers de femmes qui ne se marieront sans doute pas avant la cinquantaine (figure 14).

(13) Les célibataires doivent être entendus au sens légal : personnes n'ayant jamais été mariées. Les non-célibataires sont donc des personnes ayant été mariées (légalement) au moins une fois. Les proportions de célibataires aux différents âges donnent ainsi une indication sur la plus ou moins grande précocité des mariages. 
Dans la moitié orientale du continent, la Hongrie illustre le cas de pays où le mariage est resté jusque récemment précoce et fréquent, avant de s'écarter brutalement de ce modèle traditionnel autour des années 1980. Seules $30 \%$ des femmes nées vers 1960 n'étaient pas encore mariées à 20-24 ans et moins de $10 \%$ étaient toujours célibataires à 50 ans. En un petit nombre de générations, le célibat des jeunes a rejoint l'étonnant niveau de $90 \%$, comme en Suède et aux Pays-Bas, et la fraction de celles qui ne se marieront pas avant 50 ans annonce aussi, avec quelques décennies de retard, une croissance rapide. C'est peut-être la fin d'une spécificité forte de l'Europe de l'Est. L'évolution en Roumanie est moins radicale qu'en Hongrie, mais son orientation est la même.

Figure 14. Proportion de femmes célibataires aux différents âges dans les générations aux Pays-Bas et en Suède, Hongrie et Roumanie
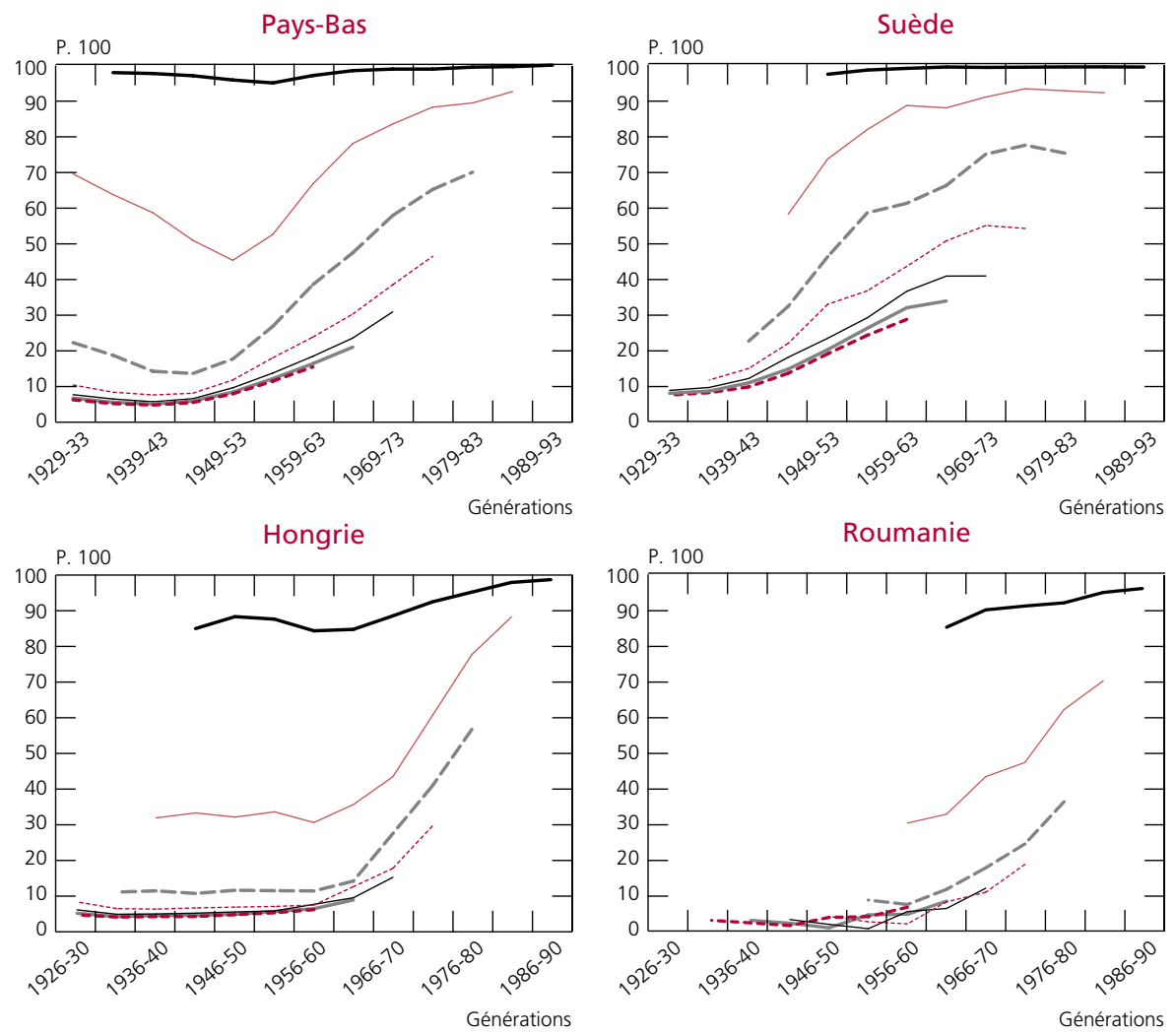

$$
-15-19-20-24---25-29---30-34-35-39-40-44 \quad---45-49
$$

Source : Calculs de l'auteur à partir de la base de données d'Eurostat. 
Quelles sont plus précisément les tendances pour ces quatre pays ${ }^{(14)}$ ? La proportion de femmes qui ne se marieront pas avant 50 ans est partout en forte hausse, passant de moins de $10 \%$ à environ $30 \%$ en vingt ou trente générations (figure 15). Lévolution est la même partout, mais avec un net décalage temporel : d'abord la Suède, puis les Pays-Bas une quinzaine de générations plus tard, enfin la Hongrie et la Roumanie, avec dix années supplémentaires de retard.

Lâge moyen des femmes au premier mariage est passé de 24 à 31 ans en Suède en une trentaine de générations. C'est un bouleversement d'une ampleur impressionnante, mais le mouvement semble proche de son terme. L'évolution en cours aux Pays-Bas, avec une quinzaine d'années de décalage, se situe à des âges plus jeunes (23 à 28 ans) : le pronostic d'une convergence entre les deux pays est difficile à établir. À l'Est du continent, le mouvement vers un mariage plus tardif reproduit en Hongrie ce qu'on a enregistré à l'Ouest un peu plus tôt. La hausse est plus modérée en Roumanie, laissant subsister à ce jour une originalité orientale, même si c'est dans le cadre d'un report généralisé des âges au mariage (figure 15).

Figure 15. Évolution de la fréquence du célibat à 50 ans et de l'âge moyen des femmes au premier mariage selon les générations aux Pays-Bas, en Suède, Hongrie et Roumanie
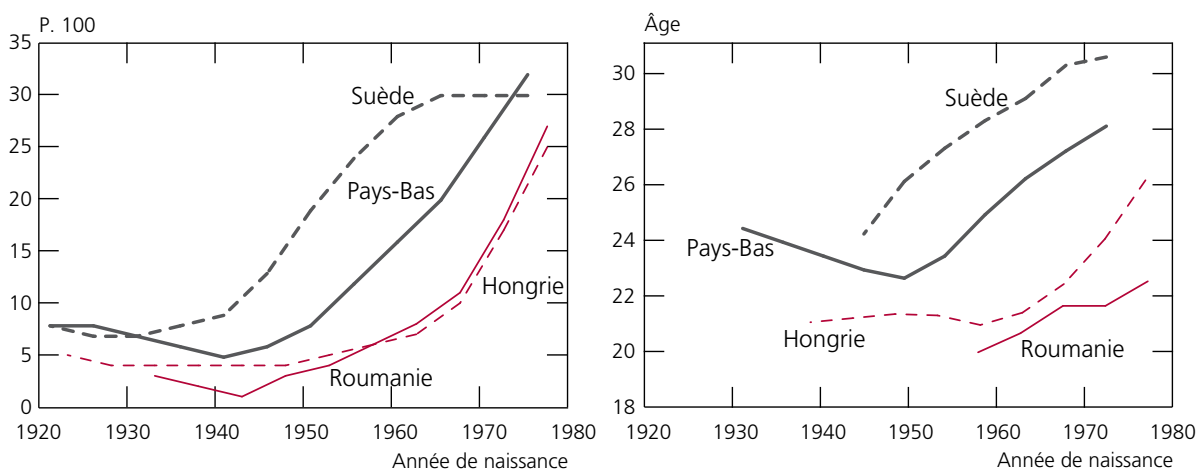

Source: Calculs de l'auteur à partir de la base de données d'Eurostat (extrapolation à quotients de nuptialité constants).

\section{Mariage, divorce, cohabitation}

La montée du célibat et l'augmentation de l'âge moyen au premier mariage témoignent d'un phénomène plus large de déclin de l'institution matrimoniale. Il se traduit aussi par une montée du divorce à peu près partout en Europe, une moindre fréquence des remariages après divorce ou veuvage, et un développement d'unions moins formelles, généralement regroupées sous le terme de cohabitation.

(14) Les quotients de nuptialité aux âges avancés sont supposés constants à leur niveau immédiatement antérieur à 2010. 
En matière de divorce, la hausse au cours des quarante dernières années est commune à l'ensemble du continent. Toutefois, sa vigueur a été plus soutenue au Nord et à l'Ouest où des changements législatifs ont accompagné le mouvement, rendant l'accès à la procédure généralement plus aisé. Les divorces représentent aujourd'hui $40 \%$ à $50 \%$ des mariages contre $10 \%$ à $20 \%$ vers 1970 . Dans les pays méditerranéens comme l'Italie ou l'Espagne, la hausse a été beaucoup moins marquée et la fréquence reste de l'ordre de $10 \%$, un fossé important s'étant creusé avec le reste de l'Europe occidentale. Les anciens pays socialistes forment un ensemble hétérogène : la divortialité est relativement élevée et augmente régulièrement en Hongrie, en République tchèque et dans les pays Baltes ; elle est plus modérée en Pologne, Roumanie, Bulgarie et Slovaquie, même si elle connaît depuis peu une forte hausse. La fréquence du divorce en Europe centrale et orientale représente aujourd'hui entre $20 \%$ et $50 \%$ des mariages (Sardon, 2006 ; Sobotka et Toulemon, 2008).

Parallèlement au recul du mariage et à la montée du divorce, les unions informelles se sont multipliées. Dans la moitié occidentale du continent, la situation contemporaine est cependant très disparate (figure 16). Aux recensements de 2000-2001, la proportion de femmes vivant en couple sans être mariées à 20-34 ans, s'échelonne d'environ $5 \%$ au Sud jusqu'à $25 \%$ à $30 \%$ au Nord ou dans les pays de l'Ouest atlantique, ceux de l'intérieur du continent étant dans une position intermédiaire (10\% à $15 \%$ ). L'Europe orientale est plus homogène, la cohabitation n'étant nulle part très diffusée (entre $2 \%$ et $12 \%$ ), sauf en Estonie, proche sur ce point des pays nordiques (Sobotka et Toulemon, 2008).

Cette géographie pourrait suggérer que la cohabitation est plus courante dans les pays où le mariage a reculé depuis longtemps et profondément, les unions informelles étant alors un substitut des unions légales. Cependant, il n'y a pas de corrélation négative systématique entre la proportion de femmes mariées à 20-34 ans et la proportion de femmes cohabitantes au même âge (figure 16A) : par exemple, l'Irlande, la Slovénie ou l'Espagne combinent une fraction peu élevée de femmes mariées à une proportion assez faible de cohabitantes.

Beaucoup plus nette est la relation négative entre la fraction de jeunes femmes vivant encore au domicile parental et la proportion de cohabitantes (figure 16B). Dans les pays méridionaux, un maintien fréquent au foyer des parents s'accompagne d'un recours faible à la cohabitation hors mariage, alors que c'est l'inverse dans des pays nordiques comme le Danemark et la Finlande, où le départ du foyer parental est beaucoup plus précoce. L'Europe orientale affiche des comportements voisins de ceux décrits pour le Sud, sans doute en raison de l'importance donnée à la solidarité familiale et des difficultés à disposer d'un logement indépendant. À l'Ouest, les pays atlantiques sont proches des pays nordiques, et les continentaux de l'Europe centrale.

La relation établie par comparaison entre les pays à un moment donné confirme ce que montrent les études au fil du temps : la vie au domicile des 
Figure 16. Relation entre la proportion de femmes cohabitantes non mariées à 20-34 ans et la proportion de femmes mariées (A), et la proportion de femmes vivant chez leurs parents (B) en 2000-2001 dans divers pays européens

A. Femmes mariées et femmes cohabitantes
B. Femmes chez les parents et femmes cohabitantes
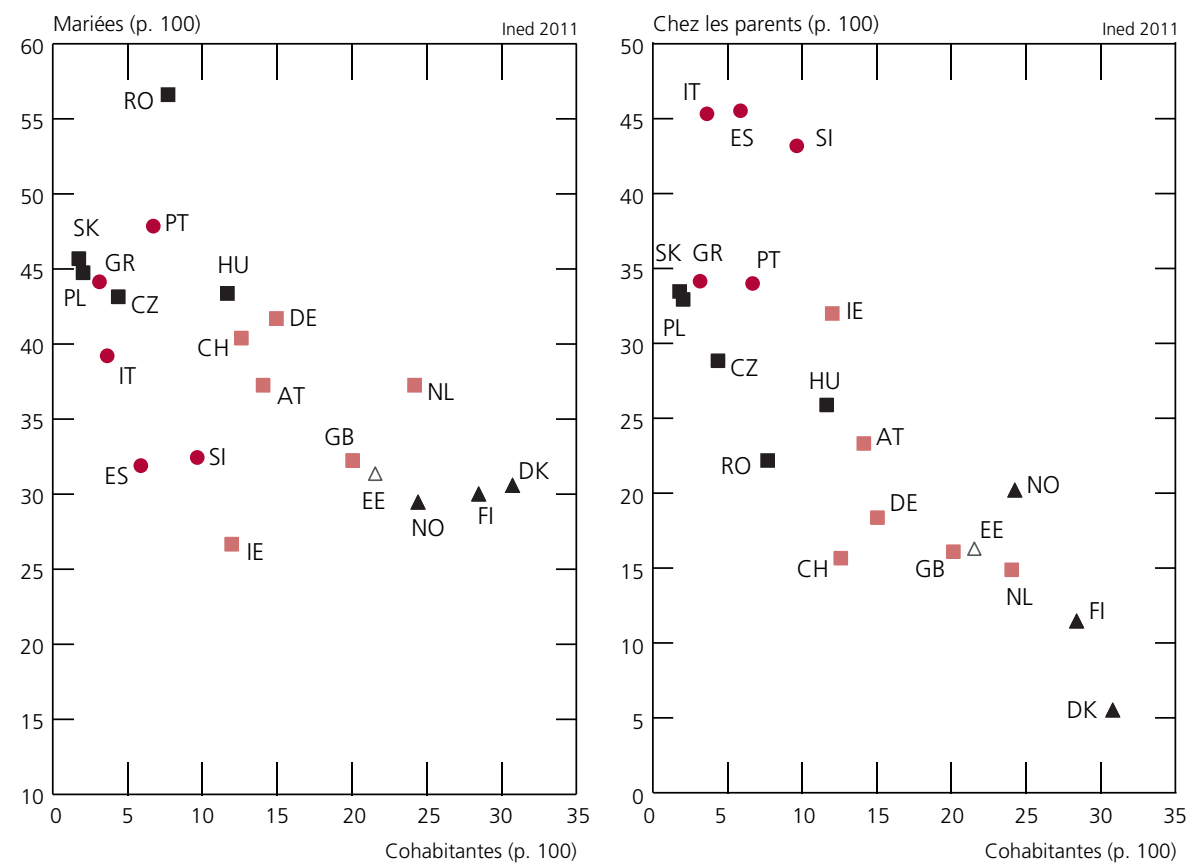

$\Delta$ Nord Ouest $\quad$ Sud $\quad$ Centre $\triangle$ Est

Source : Sobotka et Toulemon, 2008, à partir de la base de données d'Eurostat.

parents se prolonge et l'entrée en union est retardée, en liaison avec l'allongement de la vie étudiante et la difficulté croissante de trouver emploi et logement à la fin des études (Corijn et Klijzing, 2001).

\section{Fécondité hors du mariage et dans le mariage}

Le contraste entre une fécondité hors mariage forte et peu différenciée de la fécondité dans le mariage, et une fécondité hors mariage encore rare, se lit particulièrement bien dans le devenir des grossesses commencées hors mariage. Parce qu'elles sont généralement suivies de la légalisation de l'union cohabitante, les naissances hors mariage sont encore rares dans certains pays (tableau annexe A.5). C'était le cas systématiquement en Europe occidentale dans les années 1950 voire 1960 ; c'est aujourd'hui encore la situation de pays comme la Pologne, retenue ici à fin d'illustration. Au début des années 1990, 7 grossesses sur 10 de parents non mariés aboutissaient à un mariage avant l'accouchement de la mère. Malgré son recul régulier, cette proportion reste aujourd'hui supérieure à 4 sur 10 (figure 17). 
En France ou en Angleterre, la « nuptialité des femmes enceintes » ${ }^{(15)}$ était supérieure à 60 \% dans les années 1960 ; elle n'était déjà plus que de 40 \% vers 1980 ; elle continue aujourd'hui de reculer, bien qu'inférieure à $10 \%$. La fécondité hors mariage représente dans ces pays près de la moitié de la fécondité générale. L'Autriche offre un exemple de niveau intermédiaire.

Dans tous les cas, le souci du statut légal de l'enfant à naître provoque de moins en moins de réaction de la part des parents. Toutefois, le maintien de différences considérables dans la fréquence du recours au mariage pendant la grossesse induit une très forte hétérogénéité des fécondités hors mariage et traduit une acceptabilité très inégale du phénomène dans les sociétés européennes.

Figure 17. Évolution de la nuptialité des femmes enceintes depuis 1980 dans quelques pays d'Europe

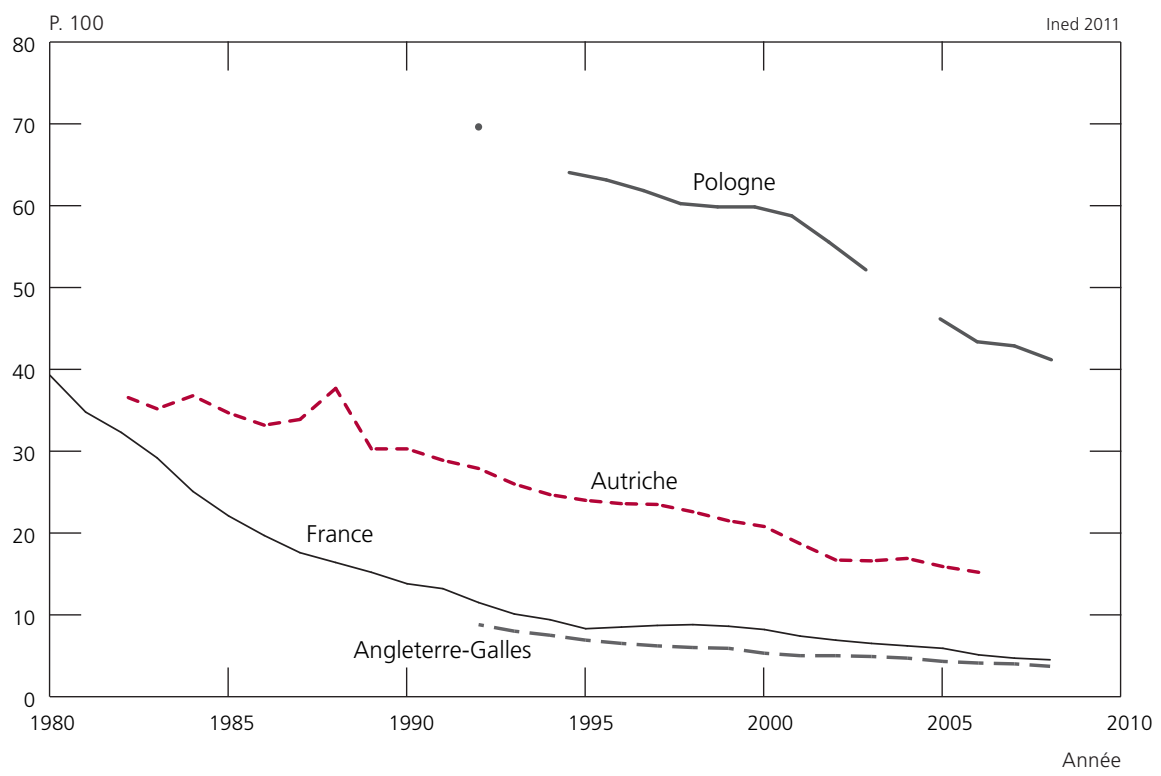

Note : La nuptialité des femmes enceintes est calculée de la façon suivante :

Naissances avant 8 mois de mariage / (naissances hors mariage + naissances avant 8 mois de mariage). Source : Calculs de l'auteur à partir des données de chaque pays.

\section{Fécondité générale et fécondité hors du mariage}

L'évolution conjuguée de la fécondité générale et de sa fraction hors mariage depuis 40 ans dans les pays d'Europe aboutit aujourd'hui à ce constat apparemment paradoxal : la fécondité des années récentes est la plus élevée dans les régions où la part des naissances hors mariage est la plus forte, par exemple au Nord du

(15) Le groupe des femmes enceintes sans être mariées est composé de celles qui vont le rester jusqu'au terme de leur grossesse en accouchant d'un enfant hors mariage et de celles qui se marient avant le terme (mariage avec « conception prénuptiale »). La nuptialité des femmes enceintes est le ratio du second sous-groupe (celles qui se marient) à l'ensemble (celles qui sont enceintes). 
Figure 18. Indicateur conjoncturel de fécondité et proportion de naissances hors mariage en 2005

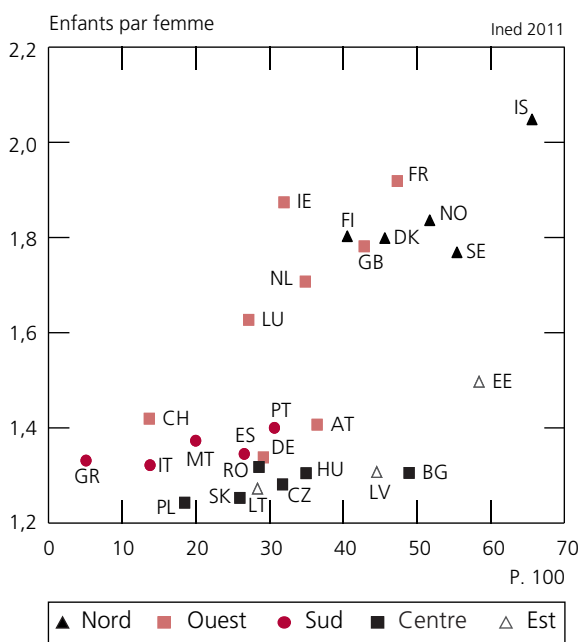

Source : Calculs de l'auteur à partir de la base de données d'Eurostat. continent ; c'est l'inverse dans les pays méridionaux, qui combinent fécondité générale et fécondité hors mariage faibles. L'Europe de l'Ouest est dans une position intermédiaire. Il en va un peu différemment dans la moitié orientale du continent, où la fécondité générale est partout basse, voire très basse, et où la part des naissances hors mariage est généralement modérée, sauf en Estonie, Lituanie, Bulgarie et Slovénie où la fécondité hors mariage reste élevée (figure 18).

Si nous prenons la proportion des naissances hors mariage comme un indicateur du recul qu'a connu l'institution du mariage en Europe au cours des dernières décennies, le plus prudent des constats est qu'il n'y a pas d'association claire et systématique

entre le degré de « désinstitutionalisation » des différents pays et le niveau de fécondité. Déclin du mariage et basse fécondité relèvent sans doute de deux schémas d'explication distincts.

\section{Comment interpréter ces différents schémas de fécondité?}

\section{La deuxième transition démographique}

Les interprétations les plus couramment admises des évolutions en matière familiale en Europe au cours des quarante dernières années sont regroupées sous le concept global de « seconde transition démographique », une théorie proposée par Ron Lesthaeghe et Dirk van de Kaa à partir de 1986 (Lesthaeghe et van de Kaa, 1986).

La « première transition démographique » traite du recul de la mortalité et de la fécondité qui avait commencé à se dessiner à la fin du XVIII ${ }^{\mathrm{e}}$ siècle en France et qui s'est étendu progressivement au reste du monde jusqu'à nos jours.

Au cours de la seconde transition démographique qui débute dans certains pays dès les années 1960, la fécondité se fixe durablement sous le niveau de remplacement des générations, des formes diverses d'organisation domestique autres que le mariage se développent, et la fécondité devient indépendante du cadre légal de la vie en couple. La seconde transition démographique est porteuse de nouvelles questions sociales : l'accentuation du vieillissement des populations, la moindre stabilité des ménages, la prévalence de la pauvreté dans certains types de ménages, comme les familles monoparentales ou les personnes vivant seules (Billari, 2008 ; Lesthaeghe, 2001 ; McDonald, 2008). 
La première transition démographique a été associée en Europe à une phase de développement au cours de laquelle la croissance économique favorisait les aspirations matérielles, l'amélioration des conditions de vie (travail, logement, santé), la formation du capital humain (scolarisation généralisée) et la constitution d'un système social protecteur. La solidarité était un concept central. Ces évolutions étaient soutenues par une division sexuée des fonctions au sein de la famille; elles ont contribué en retour à un certain embourgeoisement de celle-ci.

À mesure que les populations occidentales sont devenues plus riches et mieux instruites, leurs préoccupations se sont détachées des besoins strictement associés à la survie, la sécurité et la solidarité. Davantage d'importance a été donnée à la réalisation et la reconnaissance de soi, la liberté de pensée et d'action (recul de la religion), la démocratie au quotidien, l'intérêt du travail et les valeurs éducatives. La seconde transition démographique est donc étroitement associée au concept de « post-matérialisme » de Ron Inglehart (1990). En conséquence, la théorie de la seconde transition démographique prévoit que ses dimensions démographiques (fécondité durablement inférieure au niveau de remplacement et extension de formes alternatives d'organisation domestique) devraient apparaître dans toutes les sociétés qui se développeront sous forme d'économies capitalistes avec des institutions démocratiques, en même temps que prévaudront des « besoins d'ordre supérieur » (Inglehart, 1990). Au niveau individuel, le choix de nouvelles formes domestiques (cohabitation, vie commune chacun chez soi, etc.) est lié au développement de valeurs individualistes et non conformistes. Cette association ne se limite pas aux pays d'Europe septentrionale et occidentale ; elle s'est étendue d'ores et déjà au Sud, au Centre et à l'Est du continent.

Dans le tableau que nous avons présenté de l'évolution de la fécondité et du mariage en Europe au cours des quarante dernières années, la théorie de la seconde transition démographique apparaît assez bien adaptée à l'explication des tendances de la nuptialité et de la vie en couple, ainsi que de leur corollaire, la constitution d'une partie de la descendance hors du mariage. Dans ces domaines, l'évolution en tache d'huile à partir du Nord puis de l'Ouest du continent, vers le Sud puis l'Est, s'accorde bien avec l'évolution des attitudes et des valeurs prédominantes dans ces sociétés. La théorie est en revanche beaucoup moins convaincante quand elle s'applique à l'évolution de la fécondité, en particulier dans sa phase récente où une stabilisation à un niveau relativement élevé, voire une reprise, se dessine au Nord et à l'Ouest du continent, alors qu'elle reste très faible et parfois encore déclinante au Sud et à l'Est. Un continuum d'évolution des mentalités ne saurait rendre compte de ces disparités (Thornton et Philippov, 2009).

\section{Vers une égalité dans les rapports de genre}

Il faut sans doute prendre en compte les transformations des rapports de genre pour mieux comprendre ces disparités de niveaux ou de tendances. Dans les pays où ces transformations ont été poussées le plus loin, elles se sont faites en deux temps bien distincts. 
Dans un premier temps, un meilleur équilibre entre hommes et femmes s'est établi dans la sphère publique (en particulier dans le domaine de l'emploi), le taux d'activité des femmes s'accroissant en réponse aux progrès de l'instruction, à la baisse de la fécondité et au prolongement de l'espérance de vie. Les femmes prennent ainsi une part croissante dans la sphère publique, réservée jusque-là aux hommes, sans que ceux-ci prennent une part équivalente dans la sphère privée, mettant sous pression les familles pour qu'elles réduisent leur fécondité. Pour les pays qui n'ont pas dépassé ce stade, en particulier au Sud de l'Europe, les bas niveaux actuels de fécondité seraient la conséquence de fortes disparités entre hommes et femmes dans les responsabilités familiales, combinées à une relative égalité entre les uns et les autres en matière économique.

Dans un second temps, à des vitesses très variables selon les pays au premier rang desquels ceux du Nord du continent, l'équilibre des genres s'améliore dans la sphère privée : travaux au sein du ménage, vie de couple et de famille, soin aux personnes dépendantes. Les familles sont renforcées, dans la mesure où les hommes contribuent directement aux tâches domestiques (au sens large), la fécondité se rapprochant alors du niveau de remplacement. Une implication plus large des hommes au sein de leur famille aurait pour conséquence une plus grande égalité des genres, mais aussi une plus forte fécondité. Vont dans ce sens les études qui font apparaître une plus forte fécondité dans les couples où les pères participent davantage à la vie familiale, par exemple en prenant une large part des congés parentaux à la naissance des enfants (Goldscheider et al., 2010).

\section{Le rôle des politiques familiales}

Ces résultats amènent à s'interroger sur le rôle des politiques visant à favoriser l'égalité entre parents des deux sexes dans leurs décisions en matière de fécondité et plus généralement, sur le rôle des politiques familiales dans les différences de niveaux et de tendances de la fécondité entre pays européens dans les dernières décennies (Gauthier, 2007).

On peut être guidé pour cela par l'observation suivante. Si on associe sur un graphique le niveau récent des indicateurs de fécondité et des taux d'activité féminine en Europe, on note aujourd'hui une corrélation positive : en moyenne, plus l'activité est forte, plus la fécondité est élevée. Ce même graphique construit dans les années 1970 ou 1980 faisait apparaître une corrélation négative : la fécondité était la plus faible dans les pays où l'activité féminine était la plus forte. Ce qui conduisait à conclure sur la difficulté de concilier maternité et emploi (Thévenon, 2008). La corrélation désormais positive pourrait bien être due au fait que les sociétés qui encouragent le plus la participation des femmes au marché du travail sont aussi celles qui, en favorisant l'égalité entre conjoints et parents au sein de la famille, concourent à une fécondité relativement élevée. Ceci suggère que les politiques familiales qui aident à concilier vie familiale et vie professionnelle, en incitant les hommes à prendre davantage de part à la première, peuvent contribuer à maintenir la fécondité proche du niveau de 
remplacement des générations, comme c'est le cas actuellement dans les pays au Nord de l'Europe ou en France (Hoem, 2008 ; Ronsen et Skrede, 2010).

M.-T. Letablier et al. (2009) ont récemment passé en revue les études qui se sont attachées à la mesure de l'impact des politiques familiales sur la fécondité. Au-delà du versement d'allocations financières, de la mise à disposition de services (crèches, écoles maternelles, etc.) ou la libération de temps pour la famille (congés parentaux, horaires flexibles, etc.), ils concluent qu'il est nécessaire de prendre en compte un ensemble cohérent de mesures complémentaires intégrant les diverses politiques d'accueil de la petite enfance et d'amélioration des conditions de vie des familles, en vue de faciliter l'exercice de la fonction parentale. Ils montrent que les politiques ayant un impact sur les décisions des couples sont celles qui se maintiennent durablement en contribuant à un climat social favorable aux familles et qui assurent un soutien cohérent et continu tout au long de l'enfance.

\section{L'espérance de vie à la naissance : des progrès inégaux}

Depuis l'après-guerre, les progrès sanitaires ont été considérables partout en Europe. Toutefois, au fil des décennies, la carte des espérances de vie européennes s'est profondément modifiée, le rythme de l'amélioration n'ayant pas été similaire pour tous les pays. En 1950, le quart nord-ouest de l'Europe devançait largement les autres régions. Au milieu des années 1960, ces contrastes s'étaient beaucoup estompés, illustration du formidable rattrapage effectué par la plupart des pays du Sud et de l'Est de l'Europe. La situation a ensuite continué à s'améliorer partout en Europe sauf dans les pays de l'Est, frappés par la crise sanitaire. Dès les années 1970, une nouvelle frontière, opposant l'Est et l'Ouest, divisait la carte européenne des espérances de vie (Meslé et Vallin, 2002a ; Caselli et Vallin, 2002 ; Monnier, 2006).

Quel que soit l'indicateur analysé (espérance de vie à la naissance ou à 65 ans, mortalité infantile ou à l'âge adulte), cette ligne de fracture est toujours bien visible en 2008. En outre, durant les dernières décennies, un processus de convergence des espérances de vie masculine et féminine s'est engagé. Les pays scandinaves ont été précurseurs de cette tendance qui s'est ensuite progressivement diffusée dans le reste de l'Europe. Mais les pays de l'Est et la plupart de ceux d'Europe centrale sont, sur ce point également, restés en marge.

\section{L'espérance de vie à la naissance dans les régions d'Europe}

L'augmentation continue de l'espérance de vie européenne dissimule d'importantes divergences entre les cinq grandes régions (le Nord, l'Est, le Centre, l'Ouest et le Sud) comme en témoigne l'évolution de leurs moyennes arithmétiques, qui regroupent chacune entre 4 (région Est) et 12 pays (région Sud).

Au début des années 1960, le Nord de l'Europe était en avance sur les autres régions où l'espérance de vie était du même ordre de grandeur, en particulier 
chez les hommes (figure 19). La décennie allait être marquée par un ralentissement des gains dans les pays scandinaves, voire une stabilisation côté masculin. Ce fléchissement des progrès dans les pays les plus avancés reflétait les difficultés rencontrées à l'époque dans la lutte contre les affections cardiovasculaires ainsi que le développement des maladies de « civilisation » (tabagisme, alcoolisme, accidents de la circulation...). Il faudra attendre la « révolution cardiovasculaire » des années 1970 pour que l'espérance de vie augmente à nouveau (Vallin et Meslé, 2010). De nombreux progrès, associant innovations médicales et changements de comportements (meilleure hygiène de vie, alimentation), s'engageront progressivement au Nord, à l'Ouest et au Sud de l'Europe. En 1985, plus rien ou presque ne distingue ces trois zones, qui ont ensuite connu une amélioration continue, notamment grâce à la lutte contre la mortalité par cancer devenue de plus en plus efficace (campagnes de prévention, adoption de politiques de lutte contre le tabagisme...). Dans ces régions, compte tenu des niveaux actuels des espérances de vie à la naissance (78 ans pour les hommes et 83 ans pour les femmes), c'est désormais aux grands âges que des progrès substantiels peuvent être réalisés.

Figure 19. Évolutions de l'espérance de vie à la naissance des hommes et des femmes dans les régions d'Europe depuis 1960.

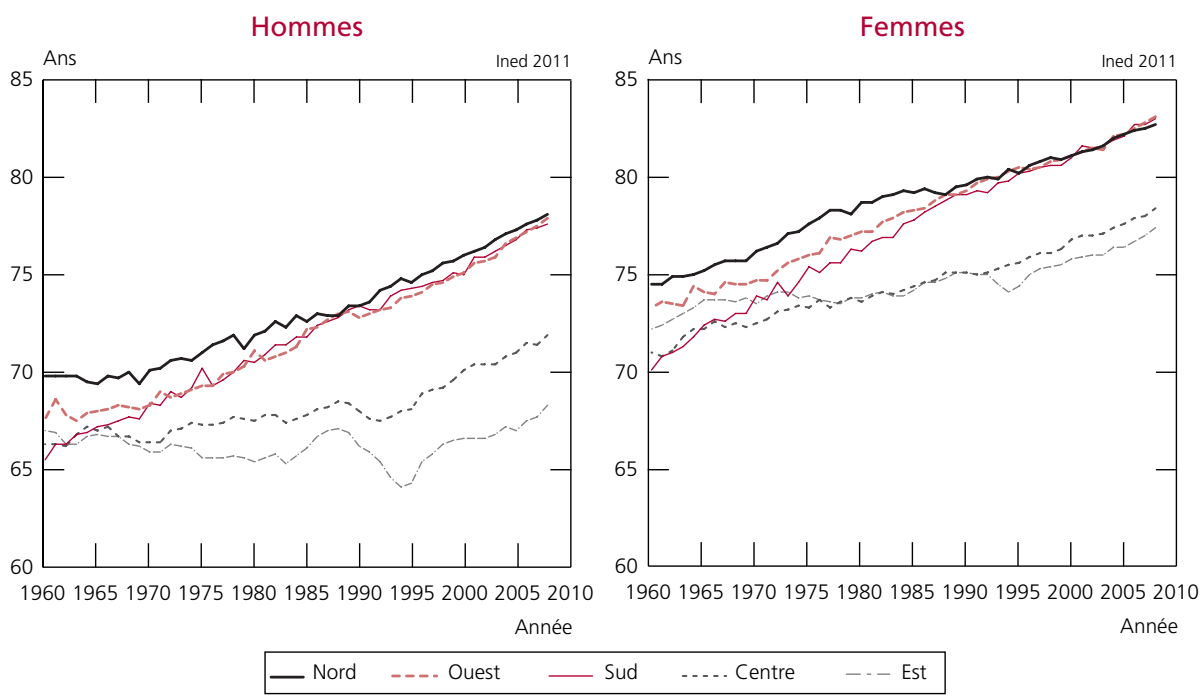

Sources: Base de données des pays développés (Ined) ; base de données Devision du Centre d'études démographiques (Moscou).

Toute autre est la situation à l'Est et au Centre de l'Europe. Au début des années 1970, les pays socialistes étaient déjà considérablement en retard sur le reste de l'Europe (Monnier et Rychtarikova, 1991 ; Monnier, 2006 ; Meslé et Vallin, 2002a ; Caselli et Vallin, 2002). Durant les années 1970-1980, ils sont restés en marge de cette marche vers la réduction de la mortalité cardiovasculaire, 
A. Avdeev et al.

Figure 20. Espérance de vie à la naissance des hommes et des femmes dans les pays d'Europe en 2008.
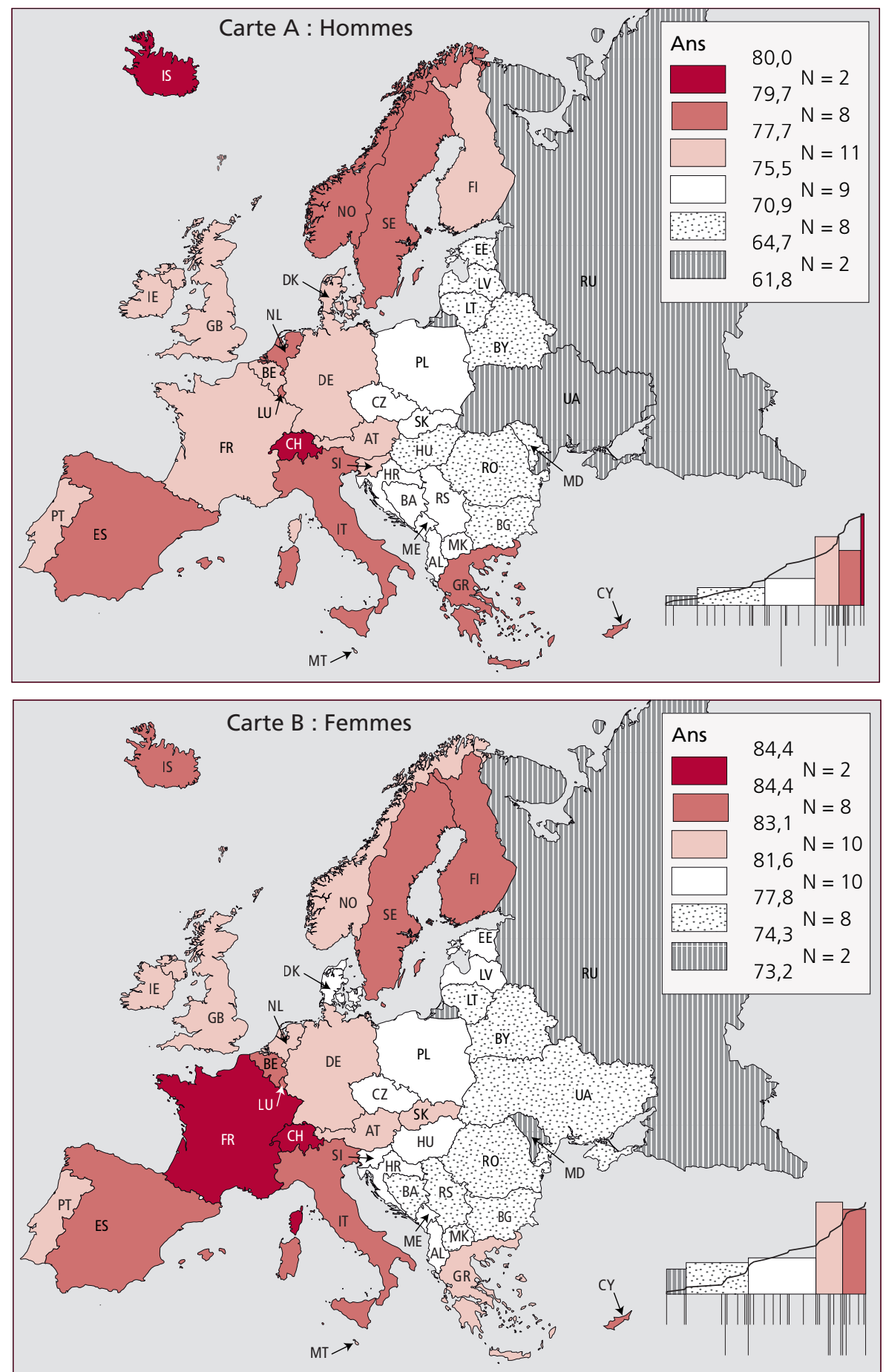

Note : Les données du Royaume-Uni concernent la période 2006-2008.

Sources: Base de données des pays développés (Ined); base de données Devision du Centre d'études démographiques (Moscou). 
l'espérance de vie s'étant stabilisée chez les hommes et ayant progressé beaucoup moins vite qu'ailleurs chez les femmes. Dans cette zone frappée par la crise et la dégradation du système de santé publique, les deux décennies suivantes seront marquées par une détérioration de la mortalité chez les hommes et des gains beaucoup plus faibles que dans les autres pays chez les femmes. En outre, au début des années 1990, l'augmentation de la consommation d'alcool par la population, en particulier masculine, a joué un rôle important dans le déclin de leur espérance de vie (Shkolnikov et al., 1995 ; Avdeev et al., 1997). Bien que depuis 1995 la durée moyenne de vie augmente à nouveau, le retard vis-à-vis du reste de l'Europe ne se réduit presque pas, les progrès étant quasiment parallèles dans les cinq sous-régions.

Malgré des histoires différentes, la carte des espérances de vie à la naissance des pays européens (figure 20) reste telle qu'elle était déjà dessinée vers la fin des années 1980, et l'opposition Est-Ouest devrait perdurer durant la prochaine décennie.

\section{Des progrès très différents selon les pays entre 1980 et 2008}

En 1980, à l'échelle des pays, l'éventail des espérances de vie à la naissance des hommes était large (tableau annexe A.6 et figure 21). Plus de dix années séparaient les extrêmes, de l'Islande (73,8 ans) à la Moldavie (62,6 ans). Une dizaine de pays, situés pour l'essentiel en Europe du Nord (Suède, Islande, Norvège), de l'Ouest (Pays-Bas, Suisse) ou du Sud (Espagne, Grèce, Chypre), avaient franchi le seuil de 72 ans. En revanche, dans tous les pays d'Europe de

Figure 21. Espérance de vie atteinte en 2008 selon l'espérance de vie atteinte en 1980

Hommes

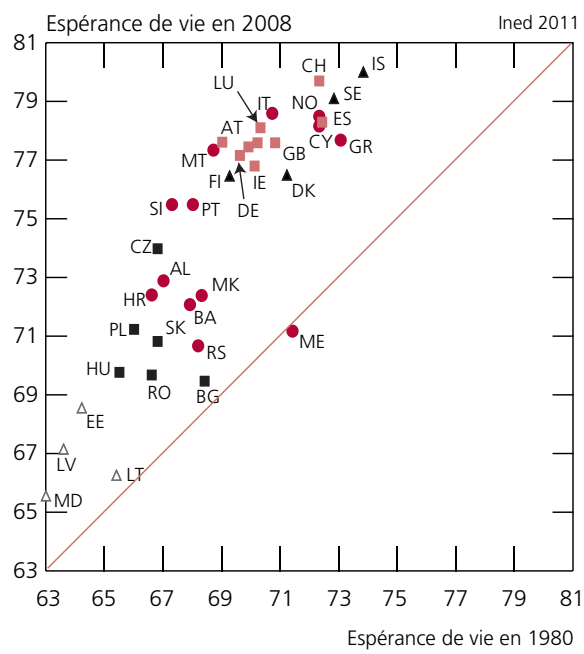

Femmes

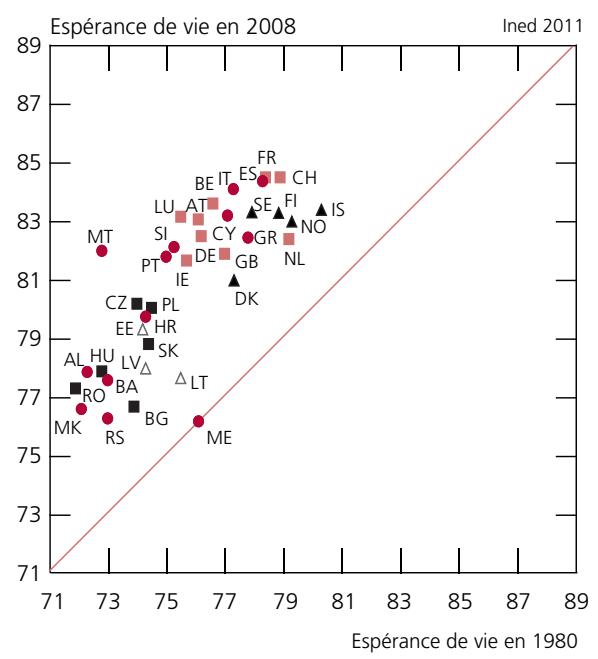

^ Nord Ouest - Sud $\square$ Centre $\triangle$ Est

Sources : Observatoire démographique européen, Eurostat. 
l'Est, en Hongrie et en Pologne, l'espérance de vie masculine restait inférieure à 66 ans. En 2008, la hiérarchie des pays ne s'est guère modifiée (coefficient de corrélation égal à 0,83 ) mais les inégalités géographiques se sont creusées, près de 15 années séparant désormais les situations extrêmes. L'Italie a rejoint les pays en tête, dans lesquels l'espérance de vie dépasse 78 ans. Avec une durée de vie moyenne inférieure à 70 ans, la Bulgarie et la Roumanie ont remplacé la Pologne dans le groupe des plus défavorisés.

De 1980 à 2008, c'est l'Europe de l'Ouest qui a enregistré les progrès les plus rapides, comblant ainsi l'avance de l'Europe du Nord : l'espérance de vie est désormais la même dans ces deux régions (78 ans, tableau 3). Quasiment partout à l'Ouest, les hommes ont gagné plus de 7,4 années de vie (avec un maximum de 8,6 ans en Autriche). Avec une échelle des durées de vie allant de 77 ans (Allemagne) à 80 ans (Suisse), cette zone est devenue en 2008 la plus homogène de toutes (figure 21). Seuls les Pays-Bas se singularisent par une augmentation moins forte et rejoignent sur ce point les pays d'Europe du Nord où les gains moyens n'ont été que de 6,2 ans. Toutefois, il y a une grande diversité des rythmes d'amélioration dans cette région : il ralentit au Danemark (5,3 ans) mais dépasse 7 ans en Finlande.

L'Europe du Sud est une terre encore plus contrastée. Non seulement les progrès y ont été d'ampleur très différente, mais les inégalités géographiques y sont beaucoup plus marquées qu'ailleurs. Le Portugal et l'Italie font partie des pays où l'espérance de vie masculine a le plus augmenté (respectivement de 7,5 et 7,9 ans), au contraire des pays de l'ex-Yougoslavie (4,4 ans en moyenne). Avec une durée de vie moyenne de 72,7 ans en 2008, ces pays sont plus proches de ceux d'Europe centrale que des autres pays du Sud où les hommes peuvent désormais espérer vivre aussi longtemps qu'en Europe de l'Ouest ou du Nord (en moyenne 77,7 ans).

En 1980, tous les pays d'Europe centrale étaient en retard par rapport à la moyenne européenne ; trente ans plus tard, ils sont encore plus distancés. Seule la République tchèque échappe à cette tendance, avec désormais une durée de vie des hommes (74 ans) comparable à la moyenne européenne. Partout ailleurs, elle est de l'ordre de 70 à 71 ans, soit parmi les plus faibles niveaux, au-dessus toutefois de ceux des pays de l'Est. C'est dans cette zone, en effet, que l'espérance de vie des hommes a progressé le moins vite (de 0,9 an en Lituanie à 4,5 ans en Estonie). Si ces pays sont dans la situation la moins favorable, ils devancent néanmoins la Russie : en 2008, l'espérance de vie à la naissance y est proche de celle observée en 1980, soit 61,8 ans. On prend ici la mesure du retard accumulé : l'espérance de vie de ces pays est inférieure à celle atteinte par la plupart des pays scandinaves en 1980.

Compte tenu de la forte corrélation entre mortalités masculine et féminine, c'est également en Europe de l'Ouest, du Nord et du Sud que se situent les pays où les femmes ont les plus longues espérances de vie, et en Europe centrale et de l'Est qu'elles sont les plus courtes. Il y a toutefois quelques exceptions dans 
le classement des pays : les pays Baltes et surtout la France sont mieux classés chez les femmes que chez les hommes, c'est l'inverse pour certains pays de l'ex-Yougoslavie (Macédoine, Monténégro et Serbie).

Tableau 3. Évolution entre 1980 et 2008 des espérances de vie à la naissance selon le sexe dans les régions européennes (moyennes arithmétiques)

\begin{tabular}{|c|c|c|c|c|c|c|c|c|c|}
\hline & & \multicolumn{3}{|c|}{ Hommes } & \multicolumn{3}{|c|}{ Femmes } & \multicolumn{2}{|c|}{ Écart F-H } \\
\hline & & 1980 & 2008 & Évolution & 1980 & 2008 & Évolution & 1980 & 2008 \\
\hline Nord & $\begin{array}{l}\text { Moyenne } \\
\text { Écart type }\end{array}$ & $\begin{array}{r}71,9 \\
1,7\end{array}$ & $\begin{array}{r}78,1 \\
1,6\end{array}$ & 6,2 & $\begin{array}{r}78,7 \\
1,2\end{array}$ & $\begin{array}{r}82,7 \\
1,0\end{array}$ & 4,0 & 6,8 & 4,6 \\
\hline Ouest & $\begin{array}{l}\text { Moyenne } \\
\text { Écart type }\end{array}$ & $\begin{array}{r}70,5 \\
1,2\end{array}$ & $\begin{array}{r}77,8 \\
0,8\end{array}$ & 7,3 & $\begin{array}{r}77,0 \\
1,4\end{array}$ & $\begin{array}{r}82,9 \\
1,0\end{array}$ & 5,9 & 6,5 & 5,1 \\
\hline Sud & $\begin{array}{l}\text { Moyenne } \\
\text { Écart type }\end{array}$ & $\begin{array}{r}69,4 \\
2,2\end{array}$ & $\begin{array}{r}74,9 \\
3,0\end{array}$ & 5,5 & $\begin{array}{r}74,9 \\
2,2\end{array}$ & $\begin{array}{r}80,3 \\
3,1\end{array}$ & 5,4 & 5,5 & 5,4 \\
\hline Centre & $\begin{array}{l}\text { Moyenne } \\
\text { Écart type }\end{array}$ & $\begin{array}{r}66,7 \\
1,0\end{array}$ & $\begin{array}{r}70,9 \\
1,7\end{array}$ & 4,2 & $\begin{array}{r}73,5 \\
1,0\end{array}$ & $\begin{array}{r}78,4 \\
1,5\end{array}$ & 4,9 & 6,8 & 7,5 \\
\hline Est & $\begin{array}{l}\text { Moyenne } \\
\text { Écart type }\end{array}$ & $\begin{array}{r}63,9 \\
1,2 \\
\end{array}$ & $\begin{array}{r}66,9 \\
1,3 \\
\end{array}$ & 3,0 & $\begin{array}{r}73,2 \\
2,8 \\
\end{array}$ & $\begin{array}{r}77,0 \\
2,6 \\
\end{array}$ & 3,8 & 9,3 & 10,1 \\
\hline Ensemble & $\begin{array}{l}\text { Moyenne } \\
\text { Écart type }\end{array}$ & $\begin{array}{r}69,0 \\
2,8\end{array}$ & $\begin{array}{r}74,5 \\
4,1\end{array}$ & 5,5 & $\begin{array}{r}75,5 \\
2,5\end{array}$ & $\begin{array}{r}80,6 \\
2,9\end{array}$ & 5,1 & 6,5 & 6,1 \\
\hline
\end{tabular}

De 1980 à 2008, l'espérance de vie à la naissance des Européennes est passée en moyenne de 75,5 ans à 80,6 ans. Avec un maximum de 84 ans (Espagne, Italie, France et Suisse) et un minimum de 77 ans (Bulgarie, Macédoine, Monténégro et Serbie), la dispersion est plus de deux fois moins importante que chez les hommes. Les contrastes entre les régions européennes sont non seulement moins accusés pour les femmes (tableau 3 et figure 21), mais ils ne se sont pas creusés depuis 1980.

C'est également en Europe de l'Ouest que les durées de vie des femmes ont progressé le plus rapidement durant la période (5,9 ans) et à l'Est le plus lentement (3,8 ans). Toutefois, elles se distinguent de leurs homologues masculins sur deux points : les gains enregistrés en Europe du Nord sont parmi les plus faibles (4 ans), et ceux d'Europe méridionale parmi les plus forts (5,4 ans). La hiérarchie des pays s'en trouve bouleversée : en 2008, tous les pays scandinaves, pour la plupart en tête en 1980, sont dépassés par l'Italie, l'Espagne, la France et la Suisse.

\section{Des gains inégaux selon les décennies et les régions}

Une décomposition par décennie montre que différentes phases de progrès de l'espérance de vie se sont succédé de façon plus ou moins rapide selon les régions (figure 22). 
Chez les hommes, durant la décennie 1980, les progrès ont été plus faibles en Europe du Nord qu'à l'Ouest et au Sud. Ils atteignent 3 ans en Belgique, Autriche ou Italie contre à peine un an au Danemark ou en Norvège. La région Nord connaît ensuite la progression la plus forte durant les années 1990 puis recommence à être largement devancée par l'Ouest, seule zone ayant connu une accélération continue des progrès d'espérance de vie. Ils y atteignent 2,7 ans en moyenne entre 2000 et 2008 et s'échelonnent de 2,2 ans (Allemagne, Royaume-Uni) à environ 3 ans (Belgique, Irlande, Luxembourg). Les gains enregistrés en Europe méridionale sont plus réguliers au fil des décennies (de l'ordre de 2 ans), hormis un léger ralentissement durant la décennie 1990, notamment en Macédoine, en Grèce et à Malte.

Figure 22. Nombre d'années gagnées en espérance de vie à la naissance au cours des décennies 1980, 1990 et 2000 selon le sexe et la région en Europe

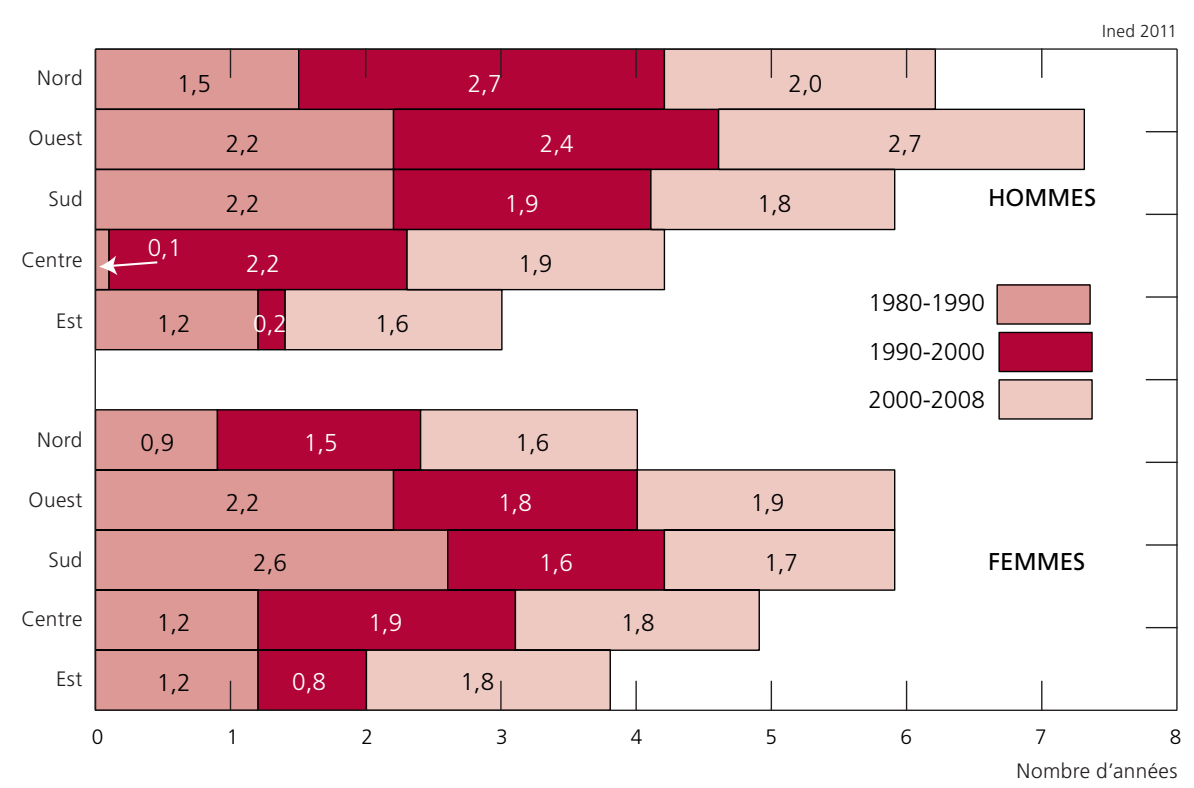

Sources: Observatoire démographique européen ; Eurostat ; voir tableau annexe A.6 pour la composition des régions à chaque date.

L'Europe centrale est la seule région où les années 1980 sont marquées par une stagnation des durées de vie et même une diminution en Bulgarie, Hongrie et Slovaquie. Durant la décennie suivante, à la suite des transformations politiques et socioéconomiques, la plupart des pays de cette région renouent avec le recul de la mortalité. L'accélération des progrès est nette (jusqu'à 4 ans en République tchèque et 3,2 ans en Pologne) et depuis 2000 ils atteignent un niveau comparable à ceux du Nord et du Sud.

En comparaison, le décalage de l'Europe de l'Est est, encore une fois, frappant. Dans cette zone, c'est durant les années 1990 que les progrès ont ralenti, 
l'espérance de vie ayant même diminué en Moldavie. Si durant la décennie suivante on observe une reprise (sauf en Lituanie), ces gains restent toutefois en deçà de ceux observés ailleurs.

En Europe de l'Est, la durée de vie des femmes (figure 22) a, comme celle des hommes, connu un ralentissement des progrès entre 1990 et 2000, mais contrairement à eux, ensuite les gains sont comparables à ceux du reste de l'Europe. En revanche, l'Europe septentrionale se distingue très nettement des trois autres régions : quelle que soit la période, les progrès y ont été moins rapides. C'est, pour l'essentiel, dans les années 1980 et 1990 que les femmes des pays de l'Ouest, et plus encore celles du Sud, ont vu leur espérance de vie augmenter plus que celles du Nord : elles ont gagné 2,6 ans dans la décennie 1980 et, hors ex-Yougoslavie, plus de 2 ans durant la suivante (contre 0,9 et 1,5 au Nord). À partir de 2000, le Nord maintient une progression plus faible que les autres régions, où les gains sont relativement homogènes.

\section{Des changements dans les évolutions des causes de mortalité}

Cette variété de rythme des évolutions et des niveaux de la mortalité doit beaucoup aux différences d'intensité des progrès effectués dans le domaine des maladies cardiovasculaires (Meslé et Vallin, 2002b). Dès 1980, et d'abord au Nord puis à l'Ouest et au Sud, cette mortalité a reculé de façon régulière : les taux de mortalité ont été divisés par 2 dans quasiment tous les pays en deux décennies (Monnier, 2006). En revanche, à l'Est et au Centre il y a stagnation, voire aggravation de la mortalité due à ces maladies jusqu'aux années 1990, et ce n'est qu'à partir de cette date qu'un déclin s'amorce (sauf en Bulgarie, Roumanie, Moldavie, Russie). Malgré cette tendance à la baisse, le niveau de la mortalité cardiovasculaire reste beaucoup plus élevé dans cette zone (le taux comparatif est environ 2 fois plus élevé qu'à l'Ouest), ce décalage étant l'une des raisons essentielles de son retard en termes d'espérance de vie sur le reste de l'Europe.

Par ailleurs, les dates auxquelles la mortalité par cancer a cessé d'augmenter sont également très variables selon les pays. Au Nord, à l'Ouest et au Sud, la hausse s'est interrompue dès les années 1980, voire plus précocement dans certains pays, alors qu'en Europe centrale et de l'Est la mortalité a continué à s'aggraver régulièrement jusqu'aux années 1990. L'Irlande, l'Espagne, la Grèce et le Portugal sont également dans cette situation, sans doute en raison d'une adoption plus tardive de dispositions contre le tabagisme. Depuis, la mortalité par tumeur a baissé partout en Europe, plus lentement toutefois que celle par affections cardiovasculaires et, par comparaison avec ces dernières, les niveaux atteints dans les diverses régions sont plus homogènes.

En revanche, de très forts contrastes s'observent pour les morts violentes (accidents, empoisonnements, suicides, homicides). Globalement, cette mortalité n'a cessé de diminuer partout en Europe depuis 30 ans, sauf dans les pays de l'Est qui, dès les années 1980, se caractérisaient par des niveaux plus élevés. La mortalité violente y a, de surcroît, connu une très forte progression 
à partir des années 1990, principalement du côté masculin. Même si ces causes de décès ont peu de poids dans la mortalité générale, elles illustrent une situation sociale particulièrement dégradée à l'Est : chez les hommes, les risques sont 4 fois plus élevés que dans le Sud, 3 fois plus que dans le Nord ou l'Ouest et 2 fois plus qu'au Centre de l'Europe (Monnier, 2006).

Partout, les femmes ont, dans un premier temps, davantage bénéficié des progrès en matière cardiovasculaire. Les hommes en ont ensuite également tiré profit mais à des rythmes différents selon les pays. De plus, la diversité d'évolution de leur surmortalité dans les autres causes de décès, en particulier cancéreuse, ont conduit les pays à une réduction plus ou moins précoce des écarts d'espérance de vie selon les sexes (Meslé, 2004 et 2006). Ce rapprochement est, certes, la conséquence du recul chez les hommes des attitudes nocives à leur santé, mais aussi de la diffusion chez les femmes des comportements masculins. Ainsi, l'un des facteurs essentiels du ralentissement de l'amélioration de la durée de vie des femmes dans les pays du Nord, en comparaison notamment de celles du Sud, pourrait être leur surconsommation de tabac (Vollset, 2008).

\section{Un rapprochement des espérances de vie des hommes et des femmes}

À l'échelle de l'ensemble des pays d'Europe, l'écart entre la moyenne des espérances de vie masculine et féminine s'est légèrement réduit entre 1980 et 2008, passant de 6,5 ans à 6,1 ans. Si, aujourd'hui en Europe, les femmes vivent partout plus longtemps que les hommes, une grande diversité existe tant dans l'intensité de l'écart que dans son évolution au cours du temps (tableau 3, figure 23).

Figure 23. Évolution de la différence absolue (en années) entre l'espérance de vie à la naissance des femmes et des hommes

A. $1980-2000$

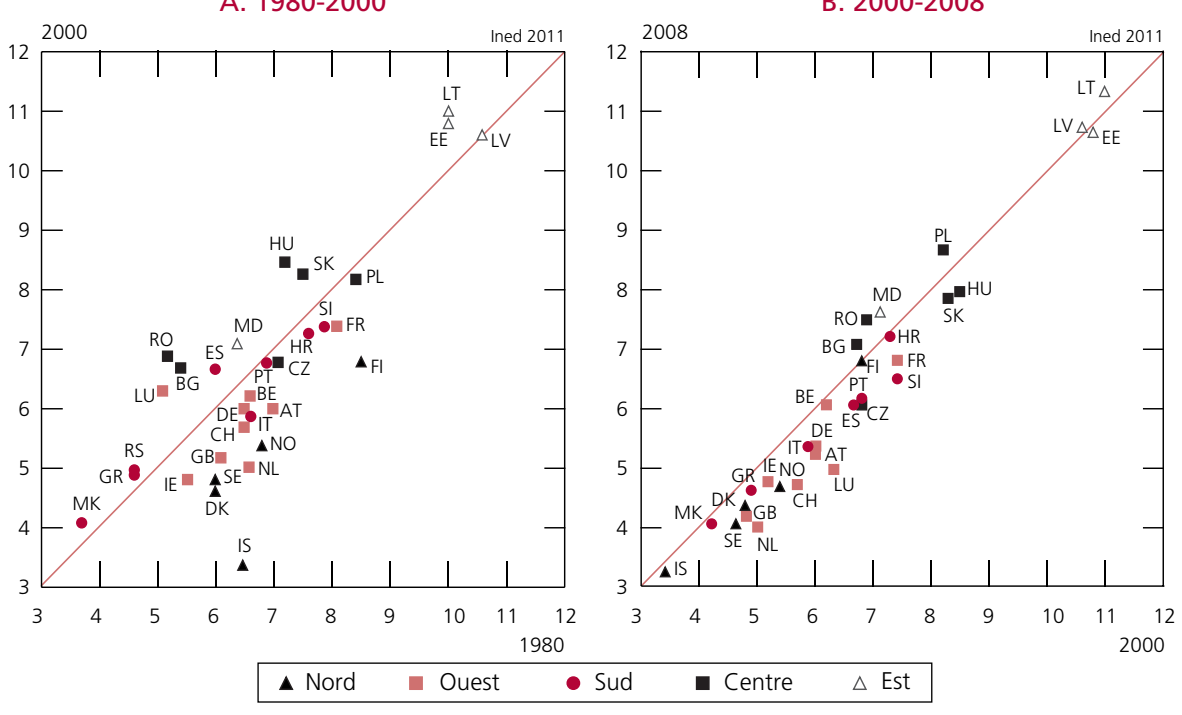

Source : Ined, base de données des pays développés 
Les pays nordiques ont été précurseurs, pour l'essentiel en raison du rapprochement des risques cardiovasculaires pour les hommes et les femmes. Les écarts, plutôt inférieurs à la moyenne européenne dès 1980 (sauf en Finlande), ont diminué régulièrement pour n'être plus que d'environ 4 à 5 ans en 2008, soit parmi les plus faibles niveaux (avec un minimum de 3,3 ans en Islande, figure 23). Il semblerait toutefois qu'en Islande et Finlande la différence se soit stabilisée depuis 2000 ; phénomène conjoncturel ou véritable retournement de tendance dans ces deux pays qui font partie de ceux où la convergence a été la plus forte et la plus précoce?

C'est un peu plus tardivement, dans la décennie 1990, que l'Europe de l'Ouest a suivi les pays septentrionaux : égal à 6,4 ans en 1980 et 1990, l'écart moyen est passé à 5,1 ans en 2008, tous les pays se situant dans cet ordre de grandeur. La France, particulièrement inégalitaire (6,8 ans), et les Pays-Bas, en situation inverse (4 ans), font toutefois exceptions.

L'Europe du Sud suit la même tendance avec, toutefois, des évolutions plus variées : diminution de la surmortalité masculine dès 1990 dans la plupart des pays, mais seulement à partir de 2000 au Portugal ; stabilité des différences en Grèce, Croatie et Macédoine. En 2008, de forts contrastes caractérisent cette région, les femmes vivant de 4,1 ans (Macédoine) à 7,6 ans (Croatie) de plus que les hommes.

En revanche, dans tous les pays d'Europe centrale, l'avantage des femmes s'est amplifié durant les années 1980 pour atteindre en moyenne près de 8 ans en 1990. Au cours de la décennie suivante, cette tendance ne s'est poursuivie qu'en Roumanie ; ailleurs, l'écart s'est stabilisé (Hongrie et Bulgarie) ou a diminué (Pologne, Slovaquie, République tchèque). Depuis 2000, il s'amplifie à nouveau dans la moitié des pays (Bulgarie, Pologne, Roumanie) et en 2008, cette région est l'une des plus inégalitaires, les femmes pouvant espérer vivre au minimum un peu plus de 6 ans que les hommes en République tchèque et jusqu'à près de 9 ans en Pologne.

Les trois pays Baltes font clairement exception, la surmortalité masculine n'y ayant cessé de s'aggraver : en 1980 comme en 2008, l'espérance de vie des femmes dépasse celle des hommes de plus de 10 ans, avec un maximum de 11,3 ans en Lituanie. Ces différences sont toutefois inférieures à celles observées en Russie (11,6 ans en 1980, 12,3 ans en 2008). Ce résultat est une autre illustration de la situation extrêmement défavorable de la mortalité masculine dans cette zone.

\section{La mortalité par âge}

L'analyse plus détaillée de l'évolution de la mortalité aux divers âges de la vie montre que, presque partout en Europe, décéder avant l'âge de 65 ans est désormais devenu rare, bien que là encore, les pays d'Europe centrale et de l'Est restent loin de cette situation. 


\section{Une mortalité infantile devenue très faible}

Depuis 1950, la baisse de la mortalité infantile a été quasiment continue partout en Europe. Seuls les pays de l'Est ont fait exception au début des années 1990 avec une légère augmentation temporaire, due à la crise sanitaire mais aussi aux changements dans la définition des naissances vivantes (Kingkade et Sawyer, 2001 ; Avdeev et Blum, 1996).

Dès 1980, la mortalité infantile était inférieure à $10 \%$ dans tous les pays d'Europe septentrionale, aux Pays-Bas et en Suisse (tableau annexe A.7). Dix ans plus tard, l'ensemble de l'Europe de l'Ouest, l'Espagne, la Grèce, l'Italie, Malte et la Slovénie se situaient nettement en dessous de ce seuil, la plupart des pays du Nord ayant franchi celui de $6 \%$. La mortalité infantile a également baissé dans le reste de l'Europe, mais en 1990 les taux restaient supérieurs à $15 \%$ dans quasiment toutes les républiques yougoslaves et tous les pays d'Europe centrale, ceux de l'Est se situant en moyenne à 13,8 \%o (tableau 4).

Tableau 4. Taux de mortalité infantile (\%) dans les régions européennes en 1980, 1990, 2000 et 2008

\begin{tabular}{|l|l|r|r|r|r|}
\hline \multicolumn{2}{|c|}{} & 1980 & 1990 & 2000 & 2008 \\
\hline \multirow{3}{*}{ Nord } & Moyenne & 7,7 & 6,4 & 3,9 & 2,9 \\
& Écart type & 0,6 & 0,8 & 0,9 & 0,6 \\
\multirow{4}{*}{ Sud } & Moyenne & 11,2 & 7,5 & 5,0 & 3,6 \\
& Écart type & 1,8 & 0,5 & 0,6 & 0,8 \\
& Moyenne & 26,1 & 14,5 & 7,6 & 5,3 \\
& Écart type & 14,0 & 8,4 & 2,9 & 2,4 \\
\multirow{5}{*}{ Est } & Moyenne & 22,7 & 16,5 & 10,3 & 6,6 \\
& Écart type & 4,3 & 5,9 & 5,0 & 2,8 \\
\hline \multirow{2}{*}{ Ensemble } & Moyenne & 20,5 & 13,8 & 11,4 & 7,2 \\
& Écart type & 9,7 & 3,8 & 4,7 & 3,4 \\
\hline
\end{tabular}

Source : Ined, base de données des pays développés; voir tableau annexe A.7 pour la composition des régions.

Même si la réduction s'est poursuivie durant les deux décennies suivantes, la mortalité infantile reste, en 2008, synonyme de fortes inégalités géographiques; les taux vont quasiment du simple au triple entre les pays du Nord ( $2,9 \%$ en moyenne) et ceux de l'Est (7,2\%o); ils restent proches de $10 \%$ en Moldavie, Roumanie et Macédoine alors qu'ils ne sont plus que de 2,5\%o en Suède et Islande, le minimum de 1,8 \%o s'observant au Luxembourg.

\section{La mortalité à l'âge adulte}

Décéder avant 65 ans est devenu très rare. Dans les conditions actuelles de mortalité, au Nord, comme à l'Ouest et au Sud de l'Europe, $85 \%$ à $90 \%$ des nouveau-nés peuvent espérer fêter cet anniversaire. Autrement dit, dans ces régions la mortalité avant cet âge ne joue plus que faiblement sur l'augmentation de l'espérance de vie à la naissance. En revanche, les pays d'Europe de l'Est ne 
sont pas dans la même situation, avec des risques de décéder avant 65 ans nettement plus élevés (dans les pays Baltes, moins de $60 \%$ des garçons nouveau-nés peuvent actuellement espérer vivre jusqu'à cet âge). En Russie, une diminution de cette mortalité au niveau des pays occidentaux se traduirait par une augmentation de plus de 6 ans de l'espérance de vie (sexes confondus).

Si l'on s'en tient à la mortalité entre 15 et 65 ans, âges auxquels les décès par mort violente et par cancer occupent une place importante dans les causes de mortalité, le désavantage des hommes des pays de l'Est est impressionnant: en 2008, leur probabilité de décéder ${ }^{(16)}$ est en moyenne trois fois plus élevée que celle de leurs homologues de l'Ouest ou du Nord (soit $399 \%$ contre respectivement $147 \%$ ot $140 \%$, tableau 5). Avec un risque deux fois plus important (291\%o), ceux du Centre sont un peu moins défavorisés. La mortalité des femmes de ces régions est également plus forte mais les situations sont plus homogènes, les probabilités moyennes de décéder s'échelonnant de 84 \%o (dans le Nord) à $165 \%$ o (à l'Est).

Tableau 5. Probabilité moyenne de décéder entre 15 et 65 ans (\%o) dans les régions européennes en 1990, 2000 et 2008

\begin{tabular}{|c|c|c|c|c|c|c|c|}
\hline & & \multicolumn{3}{|c|}{ Hommes } & \multicolumn{3}{|c|}{ Femmes } \\
\hline & & 1990 & 2000 & 2008 & 1990 & 2000 & 2008 \\
\hline Nord & $\begin{array}{l}\text { Moyenne } \\
\text { Écart type }\end{array}$ & $\begin{array}{r}207 \\
41\end{array}$ & $\begin{array}{r}162 \\
31\end{array}$ & $\begin{array}{r}140 \\
35\end{array}$ & $\begin{array}{r}114 \\
22\end{array}$ & $\begin{array}{l}98 \\
15\end{array}$ & $\begin{array}{l}84 \\
12\end{array}$ \\
\hline Ouest & $\begin{array}{l}\text { Moyenne } \\
\text { Écart type }\end{array}$ & $\begin{array}{r}216 \\
19\end{array}$ & $\begin{array}{r}176 \\
16\end{array}$ & $\begin{array}{r}147 \\
18\end{array}$ & $\begin{array}{r}112 \\
13\end{array}$ & $\begin{array}{r}96 \\
9\end{array}$ & $\begin{array}{r}83 \\
8\end{array}$ \\
\hline Sud & $\begin{array}{l}\text { Moyenne } \\
\text { Écart type }\end{array}$ & $\begin{array}{r}243 \\
43\end{array}$ & $\begin{array}{r}226 \\
50\end{array}$ & $\begin{array}{r}194 \\
51\end{array}$ & $\begin{array}{r}120 \\
25\end{array}$ & $\begin{array}{r}113 \\
34\end{array}$ & $\begin{array}{l}97 \\
33\end{array}$ \\
\hline Centre & $\begin{array}{l}\text { Moyenne } \\
\text { Écart type }\end{array}$ & $\begin{array}{r}357 \\
38\end{array}$ & $\begin{array}{r}319 \\
37\end{array}$ & $\begin{array}{r}291 \\
38\end{array}$ & $\begin{array}{r}163 \\
16\end{array}$ & $\begin{array}{r}142 \\
20\end{array}$ & $\begin{array}{r}127 \\
18\end{array}$ \\
\hline Est & $\begin{array}{l}\text { Moyenne } \\
\text { Écart type }\end{array}$ & $\begin{array}{r}398 \\
15\end{array}$ & $\begin{array}{r}414 \\
19 \\
\end{array}$ & $\begin{array}{r}399 \\
34 \\
\end{array}$ & $\begin{array}{r}181 \\
32 \\
\end{array}$ & $\begin{array}{r}179 \\
40\end{array}$ & $\begin{array}{r}165 \\
33\end{array}$ \\
\hline Ensemble & $\begin{array}{l}\text { Moyenne } \\
\text { Écart type }\end{array}$ & $\begin{array}{r}267 \\
76 \\
\end{array}$ & $\begin{array}{r}241 \\
87 \\
\end{array}$ & $\begin{array}{r}213 \\
90 \\
\end{array}$ & $\begin{array}{r}131 \\
32 \\
\end{array}$ & $\begin{array}{r}119 \\
36 \\
\end{array}$ & $\begin{array}{r}104 \\
35 \\
\end{array}$ \\
\hline
\end{tabular}

Entre 1990 et 2008, la mortalité à ces âges a diminué dans la grande majorité des pays, l'intensité de l'évolution les dissociant toutefois en deux groupes (figure 24, tableau annexe A.8). Globalement, ceux dans lesquels la probabilité de décéder a le plus diminué durant la décennie 1990 ont ensuite connu un ralentissement de ces progrès. L'Italie, la Suisse, la Slovénie, l'Autriche, l'Espagne et la Norvège sont les seuls cas où l'amélioration a été forte et de même ampleur

(16) Les tables de mortalité pour tous les pays européens sont disponibles sur le site web de l'Organisation mondiale de santé (OMS) http://www.who.int/healthinfo/statistics/mortality_life_tables/ Dans nos calculs nous avons utilisé les tables de mortalité recalculées en mai 2010. 
durant les deux décennies. À l'inverse, les pays dans lesquels la progression a été plus faible dans les années 1990, situés pour l'essentiel au Sud et au Centre, ont ensuite connu une accélération de la baisse. La mortalité adulte s'est donc homogénéisée sur la majeure partie du territoire européen.

Figure 24. Évolution de la probabilité de décéder entre 15 et 65 ans pour les périodes $1990-2000$ et 2000-2008 (\%)

HOMMES

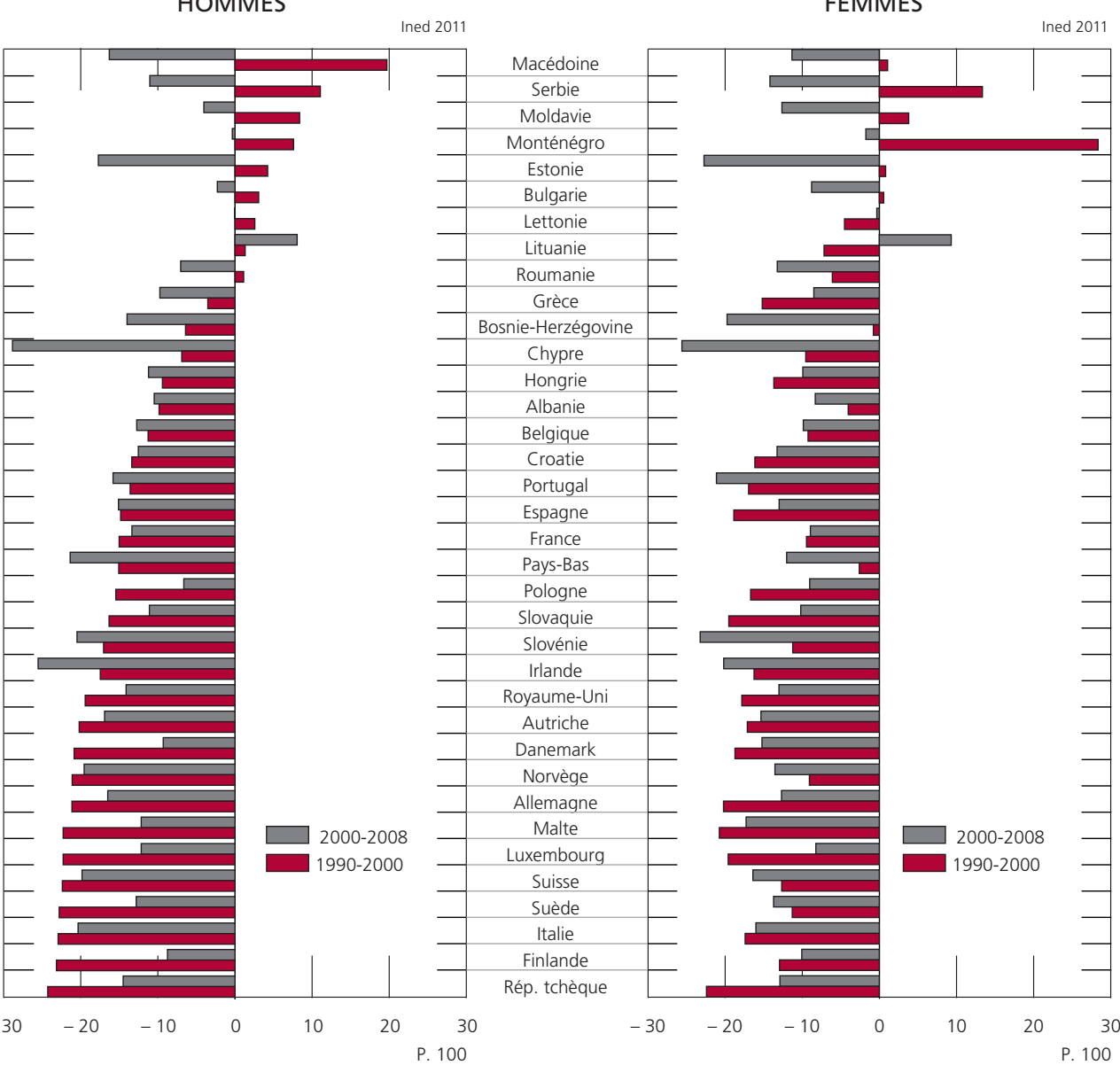

Note : Les pays sont classés selon leur évolution chez les hommes durant la période 1990-2000 Lecture : Les valeurs positives à droite signifient une augmentation et les valeurs négatives à gauche une diminution du quotient de mortalité ; le changement du quotient de mortalité dans l'intervalle entre 1990 et 2008 est la combinaison des deux valeurs qu'on calcule en respectant les signes. Source: OMS, Global Health Observatory Database, http://apps.who.int/ghodata (beta version)

Dans ce schéma général d'amélioration, 9 pays font exception. Dans 7 d'entre eux (4 pour les femmes), la mortalité a augmenté jusqu'en 2000 puis a diminué. Sur l'ensemble de la période pour les hommes, ces tendances aboutissent à un recul de la mortalité en Estonie et Roumanie, mais à une stabilité 
en Serbie et à une augmentation au Monténégro, en Moldavie, Bulgarie et Macédoine. Enfin, la Lettonie et la Lituanie ont subi une dégradation continue de la mortalité adulte masculine. Les tendances sont plus favorables chez les femmes, leur mortalité ayant globalement diminué partout, sauf au Monténégro.

En 2008, en Europe du Nord, de l'Ouest et du Sud (hormis dans les pays de l'ex-Yougoslavie) la mortalité adulte est peu élevée et remarquablement homogène. Chez les hommes, dans 5 pays seulement (Danemark, Finlande, Belgique, France, Portugal), les probabilités de décéder entre 15 et 65 ans dépassent $160 \%$, avec un minimum de $103 \%$ en Islande. Ailleurs, en revanche, les risques restent beaucoup plus forts : autour de $200 \%$ dans les pays de l'ex-Yougoslavie, de 300-350 \%o en Estonie et dans tous les pays d'Europe centrale (hormis la République tchèque) et au-delà de $400 \%$ en Lituanie, Estonie et Moldavie. La mortalité des femmes est également plus élevée dans ces régions, mais le décalage avec le reste de l'Europe est moins prononcé, les probabilités de décéder s'échelonnant de moins de 65 \%o (Espagne, Italie, Chypre) à 207 \%o (Moldavie).

\section{La mortalité après 65 ans}

Les progrès effectués en matière de lutte contre les maladies cardiovasculaires se sont traduits dans les années 1970 par une accélération du recul de la mortalité aux âges avancés. Depuis cette date, l'espérance de vie moyenne en Europe à 65 ans a augmenté de plus de trois ans et celle à 80 ans de plus de deux ans. Cette augmentation a été progressive, avec toutefois une légère diminution en 2003 en raison de la canicule exceptionnelle à l'Ouest et au Sud de l'Europe. Aujourd'hui, comme nous l'avons vu dans la plupart des pays occidentaux, la mortalité est si faible de la naissance à la fin de la vie active que les progrès ne touchent plus, pour l'essentiel, que les personnes âgées (Monnier, 2006 ; Meslé et Vallin, 2002b). Si bien que certains s'interrogent sur une possible nouvelle phase de transition sanitaire, fondée notamment sur une plus grande vigilance accordée aux plus âgés dans leur quotidien, qui permettrait d'augmenter encore la durée de vie (Vallin et Meslé, 2010).

Compte tenu du poids de la mortalité aux grands âges, il existe une très bonne corrélation entre les espérances de vie à la naissance et à 65 ans. Le classement des pays selon ces deux indicateurs est le même chez les femmes, et chez les hommes il n'existe que quelques exceptions: la Finlande et les Pays-Bas sont mieux placés en terme d'espérance de vie à la naissance qu'à 65 ans, c'est l'inverse pour la France et le Royaume-Uni.

Entre 1980 et 2008, à l'échelle de l'ensemble des pays européens pour lesquels les données sont disponibles (tableau annexe A.9), l'espérance de vie à 65 ans est passée en moyenne de 13,1 à 16,1 ans pour les hommes et de 16,3 à 19,5 ans pour les femmes. Aujourd'hui, 6 pays (Islande, Suède, France, Suisse, Espagne et Italie) atteignent 18 ans côté masculin et les quatre derniers 22 ans côté féminin. À l'autre extrême, c'est essentiellement dans certains pays de 
l'Est et de l'ex-Yougoslavie, et côté masculin dans les pays Baltes, que les niveaux sont les plus bas : de l'ordre de 13-14 ans pour les hommes et 16-17 ans pour les femmes, soit des valeurs largement dépassées dès 1980 au Nord et à l'Ouest de l'Europe.

Pour l'ensemble des pays européens, la surmortalité masculine après 65 ans est restée en moyenne identique entre 1980 et 2008, plus de 3 ans séparant les espérances de vie moyennes des hommes et des femmes aux deux dates. Toutefois, cette stabilité masque une divergence entre le Nord et l'Ouest où l'écart s'est réduit, et le reste de l'Europe où il s'est creusé (tableau 6).

Tableau 6. Évolution entre 1980 et 2008 des espérances de vie à 65 ans selon le sexe dans les régions européennes (moyennes arithmétiques)

\begin{tabular}{|c|c|c|c|c|c|c|c|c|c|}
\hline & & \multicolumn{3}{|c|}{ Hommes } & \multicolumn{3}{|c|}{ Femmes } & \multicolumn{2}{|c|}{ Écart F-H } \\
\hline & & 1980 & 2008 & Évolution & 1980 & 2008 & Évolution & 1980 & 2008 \\
\hline Nord & $\begin{array}{l}\text { moyenne } \\
\text { écart-type }\end{array}$ & $\begin{array}{r}14,1 \\
1,1\end{array}$ & $\begin{array}{r}17,6 \\
0,7\end{array}$ & 3,5 & $\begin{array}{r}18,1 \\
0,8\end{array}$ & $\begin{array}{r}20,7 \\
0,7\end{array}$ & 2,6 & 4,0 & 3,1 \\
\hline Ouest & $\begin{array}{l}\text { moyenne } \\
\text { écart-type }\end{array}$ & $\begin{array}{r}13,2 \\
0,7\end{array}$ & $\begin{array}{r}17,7 \\
0,6\end{array}$ & 4,5 & $\begin{array}{r}17,1 \\
1,1\end{array}$ & $\begin{array}{r}21,1 \\
0,9\end{array}$ & 4,0 & 3,9 & 3,4 \\
\hline Sud & $\begin{array}{l}\text { moyenne } \\
\text { écart-type }\end{array}$ & $\begin{array}{r}13,4 \\
1,6\end{array}$ & $\begin{array}{r}16,3 \\
1,8\end{array}$ & 2,9 & $\begin{array}{r}15,8 \\
1,9\end{array}$ & $\begin{array}{r}19,3 \\
2,3\end{array}$ & 3,5 & 2,4 & 3,0 \\
\hline Centre & $\begin{array}{l}\text { moyenne } \\
\text { écart-type }\end{array}$ & $\begin{array}{r}12,0 \\
0,6\end{array}$ & $\begin{array}{r}14,2 \\
0,7\end{array}$ & 2,2 & $\begin{array}{r}14,8 \\
0,5\end{array}$ & $\begin{array}{r}18,1 \\
0,9\end{array}$ & 3,3 & 2,8 & 3,9 \\
\hline Est & $\begin{array}{l}\text { moyenne } \\
\text { écart-type }\end{array}$ & $\begin{array}{r}12,4 \\
0,7 \\
\end{array}$ & $\begin{array}{r}13,0 \\
0,7 \\
\end{array}$ & 0,6 & $\begin{array}{r}15,5 \\
1,1 \\
\end{array}$ & $\begin{array}{r}16,9 \\
1,9 \\
\end{array}$ & 1,4 & 3,1 & 3,9 \\
\hline Ensemble & $\begin{array}{l}\text { moyenne } \\
\text { écart-type }\end{array}$ & $\begin{array}{r}13,1 \\
1,2\end{array}$ & $\begin{array}{r}16,1 \\
2,0\end{array}$ & 3,0 & $\begin{array}{r}16,3 \\
1,5\end{array}$ & $\begin{array}{r}19,5 \\
2,0\end{array}$ & 3,2 & 3,2 & 3,4 \\
\hline
\end{tabular}

Durant les décennies 1980 et 1990, l'avantage des femmes s'est amplifié partout en Europe, sauf dans les pays d'Europe du Nord (hormis en Norvège), au Royaume-Uni et aux Pays-Bas où un processus de convergence a débuté (figure 25). Dans la décennie suivante, cette tendance s'est poursuivie dans ces pays (sauf en Islande) et l'avantage des femmes à 65 ans a également commencé à se réduire dans l'ensemble des pays d'Europe occidentale (sauf en Finlande), plus ou moins fortement : il a à peine diminué en France et en Autriche, mais d'au moins une demi-année dans tous les autres pays de cette zone. En Europe du Sud, la Grèce, le Monténégro, Malte et Chypre ont emboîté le pas des pays occidentaux. Dans le reste de l'Europe, les écarts se sont soit stabilisés (Italie, Espagne, Portugal) soit légèrement creusés (Slovénie, Croatie). Il n’y a plus guère que dans les trois pays Baltes et certains du Centre (Bulgarie, Hongrie, Pologne, Roumanie) que l'espérance de vie des femmes à 65 ans a continué à augmenter beaucoup plus vite que celle des hommes.

Comme à la naissance, on retrouve à 65 ans la diffusion progressive, du Nord au Sud de l'Europe, du processus de convergence des espérances de vie des hommes et des femmes. 
Figure 25. Évolution de la différence absolue (en années) entre l'espérance de vie à 65 ans des femmes et celle des hommes entre 1980 et 2000 (A) et entre 2000 et 2008 (B)

A. $1980-2000$

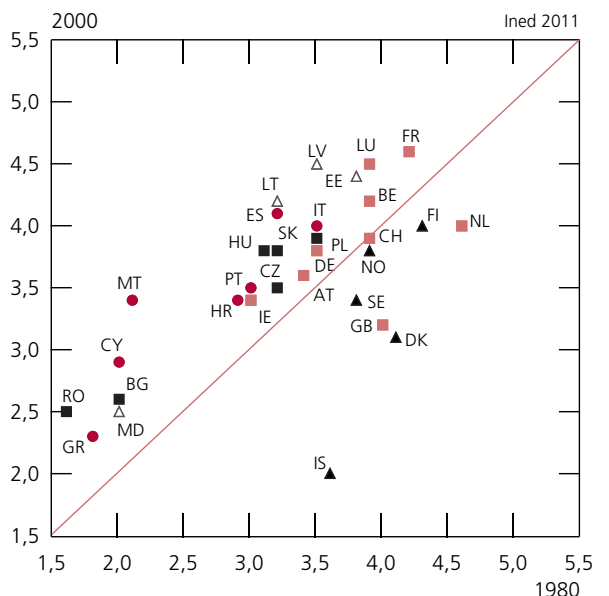

B. $2000-2008$

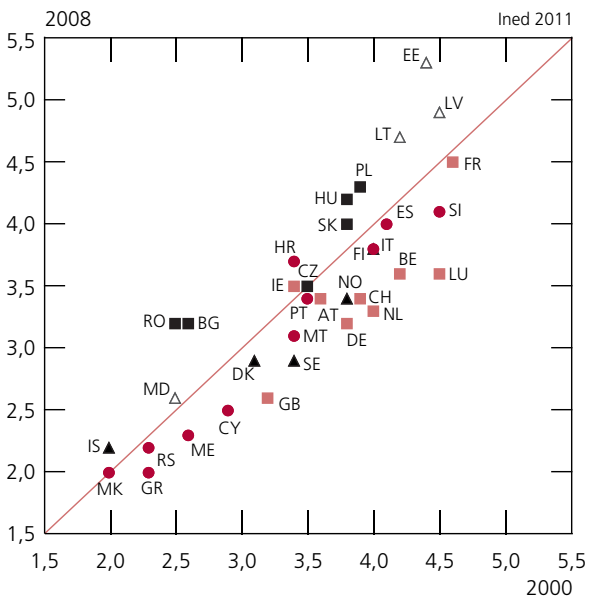

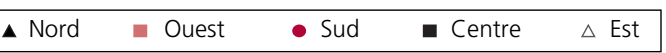

Source : Eurostat.

Toutefois, l'espérance de vie reste un indicateur imparfait de l'état sanitaire d'une population, un niveau élevé ne signifiant pas nécessairement un meilleur état de santé. Partout en Europe, les femmes vivent plus longtemps que les hommes, mais passé 65 ans, à âge égal, elles souffrent plus souvent qu'eux d'incapacités et ce, quel que soit le niveau de sévérité (Cambois et al., 2003 ; Jagger et al., 2008 ; Van Oyen et al., 2010). En conséquence, les hommes peuvent espérer vivre la majeure partie de leur vie d'après 65 ans sans incapacité (soit 8,7 ans sur près de 16 ans en moyenne en 2007) alors que c'est l'inverse pour les femmes (9 ans sur 19 ans).

Les espérances de vie avec ou sans incapacités des hommes et des femmes sont très corrélées (coefficient de 0,97 ) mais la dispersion géographique de ces indicateurs ${ }^{(17)}$ est beaucoup plus forte que celle des espérances de vie elles-mêmes. À 65 ans, un écart de l'ordre de 5 ans sépare les espérances de vie maximales et minimales des femmes en Europe, mais il est de plus du double pour leurs durées de vie avec incapacités : les Finlandaises peuvent espérer vivre en moyenne moins de 5 années avec des incapacités alors que dans certains pays du Sud (Italie, Portugal) ou Baltes cette durée sera de l'ordre de 14 ou 15 ans. Autre illustration de ces fortes inégalités géographiques, la part des années passées en bonne santé (figure 26 et tableau annexe A.9) s'échelonne selon les pays de $27 \%$ à $85 \%$ chez les hommes et

(17) Malgré les efforts d'harmonisation des données, cette dispersion peut, en partie, être liée à des différences d'appréciation, selon le contexte socioculturel, de la sévérité des incapacités. 
Figure 26. Proportion d'années vécues en bonne santé dans l'espérance de vie à 65 ans en 2007 pour les hommes et pour les femmes

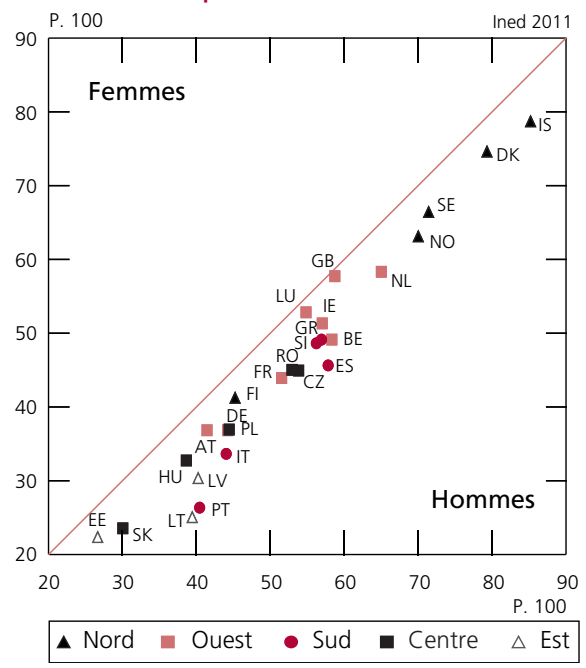

Sources: Observatoire démographique européen ; Eurostat ; tableau annexe A.9. de $22 \%$ à $79 \%$ chez les femmes. On trouve ici une illustration supplémentaire du fossé séparant le Nord dans la meilleure situation, et l'Est de l'Europe dans la plus défavorable, l'Ouest et le Sud occupant des positions intermédiaires.

Ces données montrent à quel point le vieillissement se pose et se posera demain en des termes différents selon les pays. Dans certains, la prise en charge de la dépendance sera exacerbée par le fait qu'une fraction plus importante de la population âgée vieillit en mauvaise santé.

\section{Le vieillissement démographique}

Le vieillissement démographique est généralement mesuré par l'augmentation de la proportion de personnes âgées de 65 ans et plus. Toutefois, la détermination de l'âge d'entrée dans la vieillesse reste largement arbitraire, notamment parce que ce seuil varie selon les lieux et les époques (Bourdelais, 1996). Lâge de la sénescence est de plus en plus tardif grâce à l'amélioration de l'état de santé. Mais dans les sociétés occidentales, cette étape du parcours de vie est aussi une construction sociale. L'âge de 65 ans repose en particulier sur le fait que les personnes ayant fêté cet anniversaire ont généralement quitté le marché du travail et sont donc fortement dépendantes des revenus de transfert. Cet âge social est de plus en plus dissocié de l'âge biologique de la vieillesse.

La structure par âge d'une population reflète son histoire démographique sur plus d'un siècle : la fécondité décide de l'ampleur de la base de la pyramide, la mortalité dessinant, pour l'essentiel, l'allure de son sommet. Le vieillissement peut provenir d'une diminution de la natalité qui réduit la population jeune et rétrécit la base de la pyramide (vieillissement par le bas). La précocité de la baisse de la fécondité en France au XIX ${ }^{\mathrm{e}}$ siècle a conduit ce pays à être pendant longtemps celui dont la structure démographique présentait la part de personnes âgées la plus élevée au monde. Le vieillissement peut également être la conséquence du recul de la mortalité aux âges élevés qui gonfle les effectifs de personnes âgées (vieillissement par le sommet). Actuellement, en Europe, les deux processus sont simultanés : la baisse de la mortalité aux âges avancés accélère, 
depuis l'après-guerre, l'intensité du vieillissement dû à la chute de la fécondité. Mieux que tout autre phénomène démographique, le vieillissement a un caractère largement prévisible qui nous permettra de faire état de projections à l'horizon 2040 avec une probabilité satisfaisante.

Sous l'effet de la baisse de la mortalité, la population âgée a connu, au cours des dernières décennies, une double transformation : non seulement les individus sont de plus en plus nombreux à accéder à l'âge de la retraite, mais cette période d'inactivité ne cesse de s'allonger. On distingue aujourd'hui, au sein de la population âgée, deux groupes aux modes de vie très différents, la rupture s'opérant souvent entre 75 et 80 ans. La cessation d'activité professionnelle est désormais suivie, généralement, de nombreuses années d'autonomie vécues le plus souvent en couple et en bonne santé. Puis, avec l'avancée en âge, les risques de veuvage et de dépendance augmentent, rendant plus difficile le maintien au domicile. À ces deux stades de la vie, les personnes âgées se trouvent dans des positions distinctes dans la chaîne des solidarités familiales : plus souvent aidantes qu'aidées au début de la retraite (aides financières à leur descendance, garde des petits-enfants...), elles se retrouvent généralement dans la situation inverse aux grands âges. Or la structure de la population âgée connaît également un vieillissement car la part des très âgés s'accroît. Ce processus est appelé à s'intensifier à l'avenir avec l'arrivée aux portes de la grande vieillesse des générations du baby-boom. Cette augmentation temporaire de la natalité, entre 1945 et 1975, marque durablement la forme de la pyramide des âges de la plupart des pays du quart nord-ouest de l'Europe (Monnier, 2007). L'avance en âge de ces générations nombreuses est un autre facteur d'accélération du vieillissement dans ces pays.

La carte du vieillissement de l'Europe a changé, et va continuer à changer. Du lendemain de la seconde guerre mondiale (10,3\% de personnes âgées de 65 ans et plus) jusque dans les années 1980 (13,8\%, tableau 7), l'Europe de l'Ouest avait la population la plus âgée d'Europe. Aujourd'hui, elle a été rejointe par l'Europe du Sud, les deux zones comptant environ $16 \%$ de personnes âgées, et dépassée par les trois pays Baltes (16,7\%). Dans trente ans, quasiment toutes les grandes régions européennes auront la même proportion de personnes âgées, de l'ordre de $26 \%$; seule l'Europe du Sud sera un peu plus vieillie (27\%).

Tableau 7. Proportion (\%) de personnes âgées de 65 ans et plus dans les régions européennes en 1980, 2008 et 2040

\begin{tabular}{|l|c|c|c|}
\hline & 1980 & 2008 & 2040 \\
\hline Nord & 13,4 & 15,1 & 24,8 \\
Ouest & 13,8 & 15,9 & 25,1 \\
Sud & 11,2 & 16,3 & 26,9 \\
Centre & 11,7 & 14,8 & 25,8 \\
Est & 12,3 & 16,7 & 25,3 \\
\hline Ensemble & 12,5 & 15,7 & 25,7 \\
\hline \multicolumn{2}{|l|}{ Source : Eurostat, voir tableau annexe A.10 pour les proportions par pays. } \\
\hline
\end{tabular}




\section{Le vieillissement s'accroît au Sud de l'Europe entre 1980 et 2008}

En 1980, la proportion de personnes âgées de 65 ans et plus égalait ou dépassait 12,5\% dans la moitié des pays européens (tableau annexe A.10). Les valeurs les plus élevées s'observaient essentiellement en Europe du Nord et de l'Ouest. Avec plus de 14 \% de personnes âgées, la Suède, l'Allemagne, l'Autriche, le Royaume-Uni, la Norvège, la Belgique, le Danemark et la France constituaient le peloton de tête. À l'autre extrême, l'Europe méridionale et centrale ainsi que l'Irlande et l'Islande se caractérisaient par un vieillissement moins accentué, la plupart des pays situés dans ces zones ayant moins de $11 \%$ de personnes âgées de 65 ans et plus (figure 27A).

Figure 27. Proportion de personnes âgées de 65 ans et plus (\%) en 1980 et 2008 (A) et prévisions pour 2040 (B)

dans 29 pays européens classés selon la valeur de 2008

A. $1980-2008$

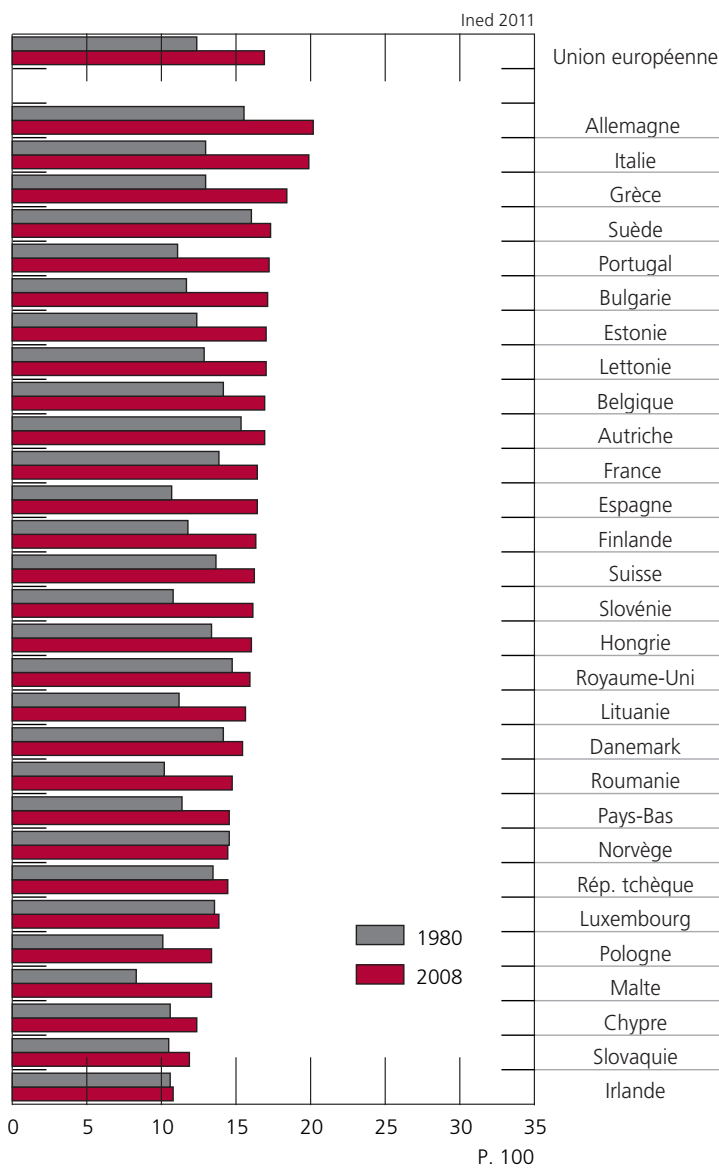

B. $2008-2040$

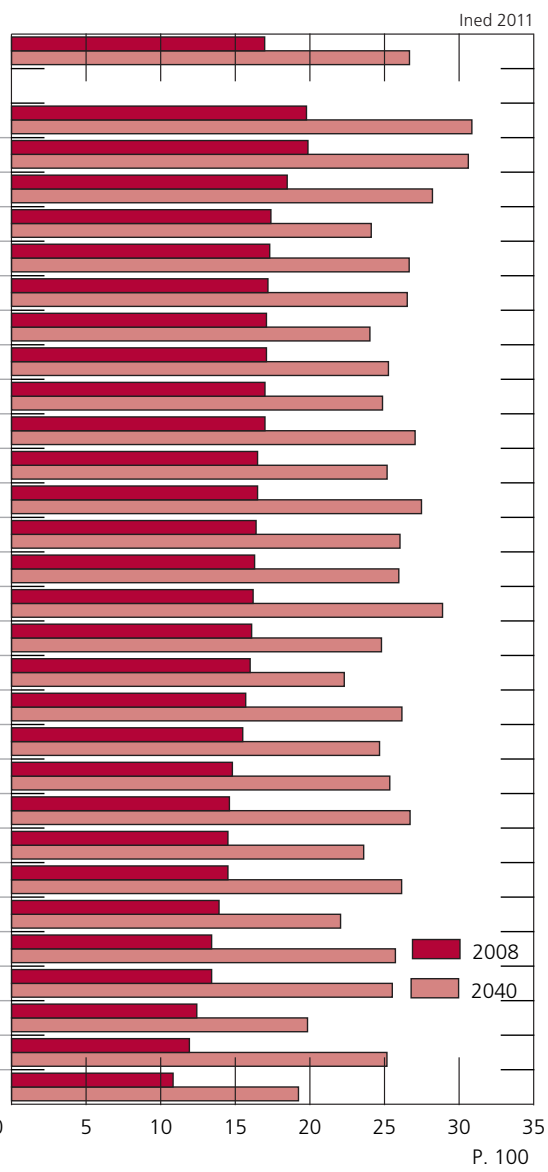

Source : Eurostat. 
Quelque trente années plus tard, en 2008, seuls 6 pays (Irlande, Islande, Moldavie, Macédoine, Slovaquie, Chypre) comptent moins de 12,5\% de 65 ans et plus. Quasiment tous les autres ont dépassé le seuil de $14 \%$, la moitié des pays se situant désormais au-dessus de 16,2\%. L'homogénéisation géographique est nette : si la population de tous les pays a vieilli, ce processus a été plus soutenu dans nombre de ceux ayant une proportion de personnes âgées plus faible au départ. Autrement dit, la carte du vieillissement européen s'est modifiée et la hiérarchie des pays observée en 2008 (figure 28) ne correspond plus que faiblement à celle observée 30 ans plus tôt (coefficient de corrélation de 0,56).

Avec 20,1 \% de personnes âgées de 65 ans et plus, l'Allemagne occupe désormais la tête du classement avec l'Italie (20\%), suivies par la Grèce (18,6 \%), le Portugal (17,4\%), la Bulgarie, la Lettonie, l'Estonie, la Serbie et la Croatie $(17,2-17,3 \%)$. Si la Suède (17,5 \%), la Belgique et l'Autriche (17,1 \%) font toujours partie des pays les plus vieillis, dans les autres pays d'Europe septentrionale et occidentale (Royaume-Uni, Danemark, Pays-Bas, Norvège), la proportion de personnes âgées est désormais comparable, voire inférieure à la valeur médiane.

Figure 28. Proportion (\%) de la population âgée de 65 ans et plus dans les pays d'Europe en 2008

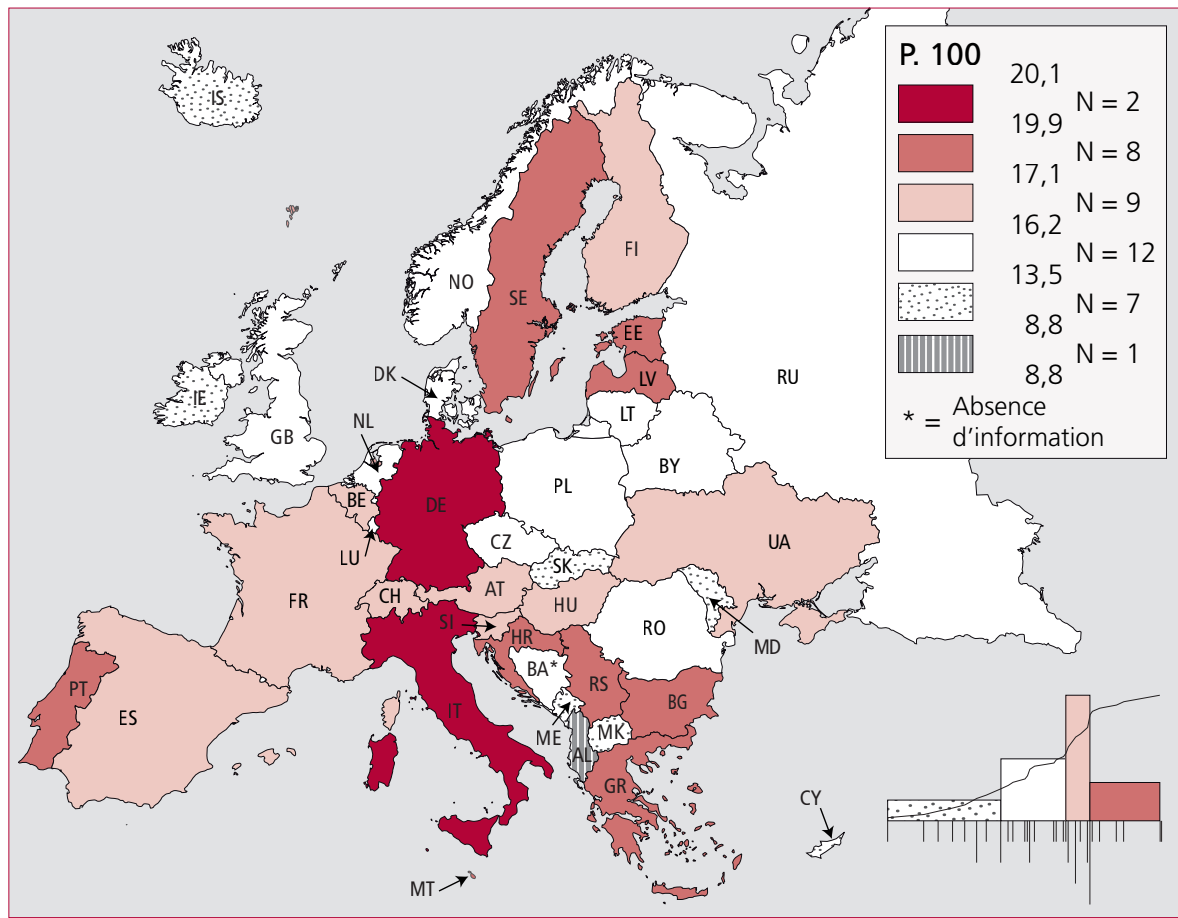

Sources : Eurostat ; ONU (2010). 
Ce changement de classement est la conséquence des variations dans l'intensité des évolutions de la fécondité et de la mortalité analysées précédemment : elles se traduisent par des rythmes d'évolution des populations jeunes, adultes et âgées différents selon les pays (figure 29).

Figure 29. Taux de croissance des effectifs de la population par grand groupe d'âges entre 1980 et 2008

dans 28 pays des cinq régions européennes (\%)

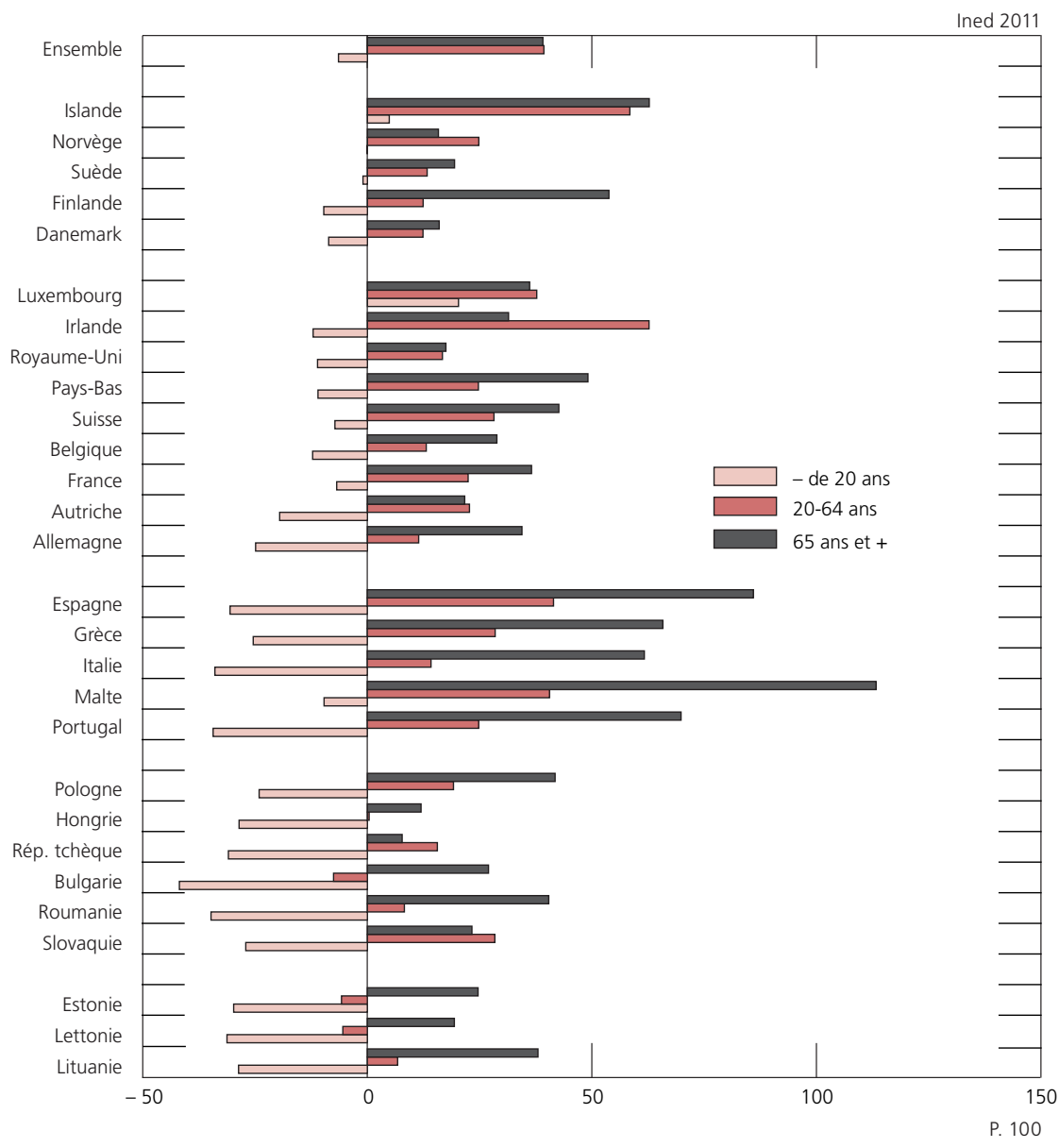

Source : Eurostat.

La plus forte progression du vieillissement dans les pays du Sud de l'Europe tient au cumul du plus fort rétrécissement de la base de leur pyramide - effondrement récent de la fécondité - et du plus fort gonflement de leur sommet - plus forts gains d'espérance de vie à 65 ans -, l'exemple type étant l'Italie. Sa population de moins de 20 ans a diminué de près de $35 \%$, celle des plus de 65 ans a augmenté de près de $62 \%$. 
La plupart des pays d'Europe centrale et les trois pays Baltes ont subi des diminutions tout à fait comparables des effectifs de moins de 20 ans (de plus de 30 \% en Bulgarie, Lettonie, République tchèque ou Roumanie). En revanche, le rythme de croissance des effectifs des plus âgés est moins soutenu (de $40 \%$ en Pologne à environ 10 \% en Hongrie et République tchèque), en raison de conditions de mortalité moins favorables.

Par comparaison, dans les pays d'Europe de l'Ouest et du Nord, où le vieillissement était le plus prononcé initialement, l'intensité des évolutions est beaucoup moins accentuée, tant à la base qu'au sommet des pyramides : les effectifs de moins de 20 ans n'y ont que faiblement diminué (au maximum $12 \%)$ et seuls 3 pays (Pays-Bas, Finlande, Islande) ont connu une croissance forte (50\% ou plus) de leur population âgée. L'Allemagne se singularise : c'est à la forte décroissance de sa population jeune (-25\%), liée à sa faible fécondité dès les années 1970, qu'elle doit son vieillissement soutenu durant la période.

\section{Une accélération du processus est attendue entre 2008 et 2040}

Les projections élaborées par Eurostat (Giannakouris, 2008 ; Goll, 2010) prévoient une accélération du vieillissement : en 2040, la part des 65 ans et plus dans la population dépassera $20 \%$ dans tous les pays sauf en Irlande (19,4\%), et dans la moitié d'entre eux elle sera supérieure à 25,7\%. Le tableau 8 montre qu'en 28 ans (entre 1980 et 2008), la proportion de personnes âgées de 65 ans et plus n'avait été multipliée par plus de 1,5 que dans 5 pays. Durant la période 2008-2040, sur une durée à peine plus longue (32 ans), ce pourrait être le cas de 26 pays (sur 29), Malte, la Pologne et la Slovaquie connaissant même un doublement de cette proportion.

Tableau 8. Coefficient multiplicateur de la proportion de personnes âgées de 65 ans et plus dans 29 pays européens entre $1980-2008$ et 2008-2040

\begin{tabular}{|c|c|c|}
\hline Coefficient & $1980-2008$ & $2008-2040$ \\
\hline 1 & Irlande, Luxembourg, Norvège & \\
\hline $1,1-1,2$ & $\begin{array}{l}\text { Autriche, Belgique, Chypre, Danemark } \\
\text { France, Hongrie, Royaume-Uni } \\
\text { Rép. tchèque, Slovaquie, Suisse, Suède }\end{array}$ & \\
\hline $1,3-1,4$ & $\begin{array}{l}\text { Allemagne, Estonie, Finlande, Grèce, } \\
\text { Lettonie, Lituanie, Pays-Bas, Pologne, } \\
\text { Portugal, Roumanie }\end{array}$ & Estonie, Royaume-Uni, Suède \\
\hline $1,5-1,6$ & $\begin{array}{l}\text { Bulgarie, Espagne, Italie, } \\
\text { Slovénie, Malte }\end{array}$ & $\begin{array}{l}\text { Allemagne, Autriche, Belgique, Bulgarie, } \\
\text { Chypre, Danemark, France, Finlande, } \\
\text { Grèce, Hongrie, Italie, Lettonie, } \\
\text { Luxembourg, Norvège, Portugal, Suisse }\end{array}$ \\
\hline $1,7-1,8$ & & $\begin{array}{l}\text { Espagne, Irlande, Lituanie, Pays-Bas, } \\
\text { Rép. tchèque, Roumanie, Slovénie }\end{array}$ \\
\hline $1,9-2,1$ & & Malte, Pologne, Slovaquie \\
\hline
\end{tabular}


Figure 30. Taux de croissance des effectifs de la population par grand groupe d'âges entre 2008 et 2040 dans 30 pays des cinq régions européennes

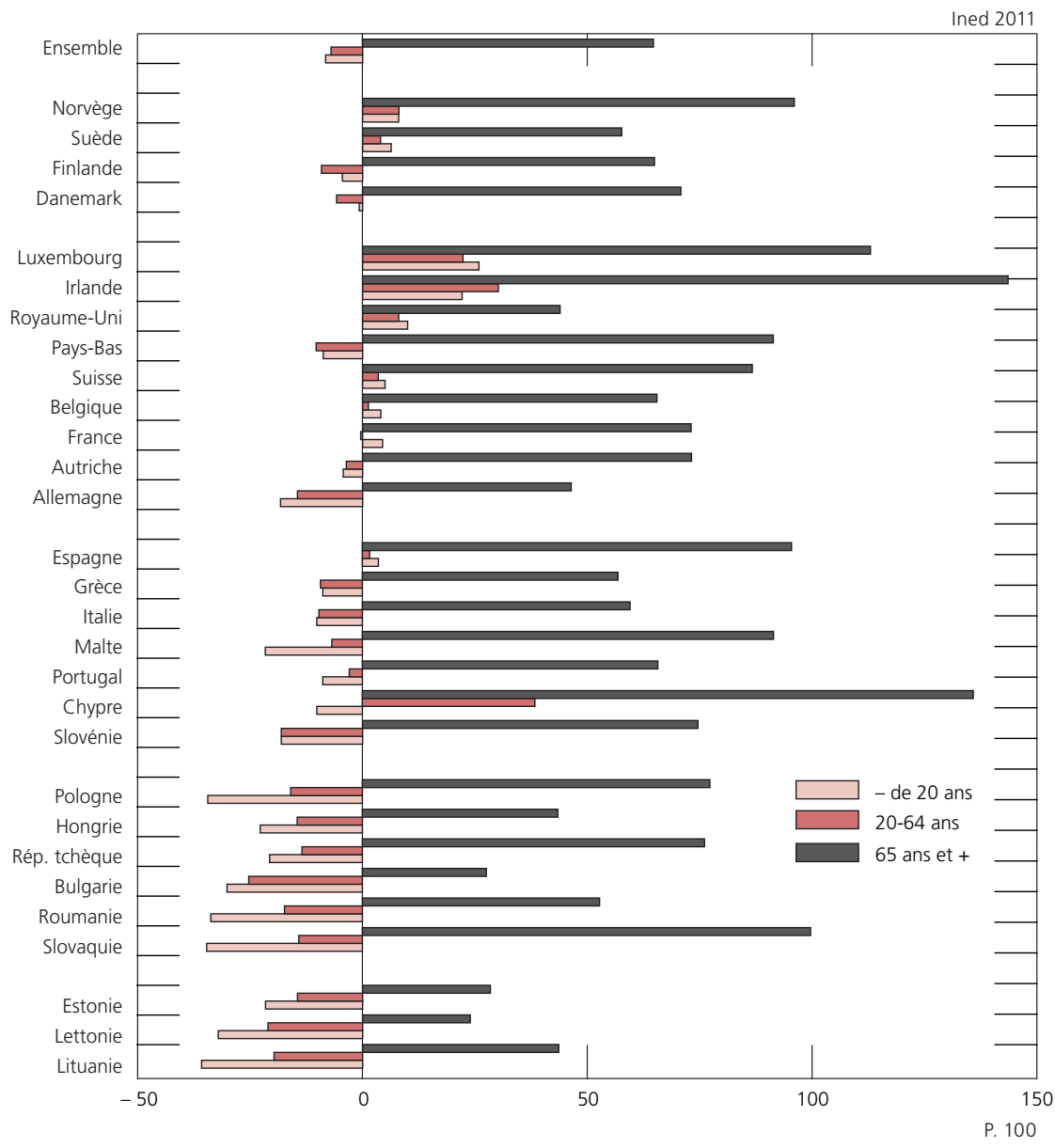

Source : Eurostat.

En outre, la hiérarchie des pays restera très largement la même (coefficient de corrélation de 0,71 ), la très grande majorité des pays progressant au même rythme vers des niveaux de vieillissement plus élevés. Avec environ $31 \%$ de personnes âgées de plus de 65 ans, l'Italie et l'Allemagne resteraient toujours en tête, suivies par la Slovénie (29\%), la Grèce, l'Espagne, le Portugal et l'Autriche (environ $28 \%$ ) (figure 27B). À l'autre extrémité de l'échelle, se trouveraient toujours des pays majoritairement situés en Europe septentrionale - l'Irlande (19,4 \%), le Luxembourg (22,2 \%), le Royaume-Uni (22,5\%) et la Norvège (23,8\%) - ainsi que Chypre (20\%).

Cette homogénéisation territoriale est en grande partie la conséquence de l'uniformité des évolutions des divers groupes d'âges, elle-même probablement 
favorisée par l'hypothèse de convergence de comportements sous-tendant ces projections européennes. Par rapport à la période précédente, l'accélération de l'intensité de la croissance des effectifs âgés est frappante (figure 30). Alors que précédemment elle ne dépassait 50 \% que dans les pays du Sud, désormais seuls les trois pays Baltes, quelques pays d'Europe centrale (Hongrie, Bulgarie), et dans une moindre mesure l'Allemagne (47\%) et le Royaume-Uni (44\%), n'atteignent pas ce taux. Dans certains pays cette progression est proche voire supérieure à 100 \% (Luxembourg, Norvège, Irlande, Slovaquie, Espagne). Poursuite de la baisse de la mortalité dans les hypothèses de projection et vieillissement des générations du baby-boom conjuguent ici leurs effets.

Autre contraste par rapport à la période précédente : à la décroissance quasi généralisée de la population jeune s'ajoute désormais, en Europe du Sud (sauf Chypre), de l'Est et du Centre, celle des effectifs adultes. L'intensité des décroissances est beaucoup plus forte dans cette dernière zone : la population adulte y diminue de $15 \%$ (Hongrie) à plus de $25 \%$ (Bulgarie), la fourchette allant de $21 \%$ (République tchèque) à près de $35 \%$ (Slovaquie) pour les moins de 20 ans. Dans les pays du Sud, la baisse de ces effectifs n'oscille qu'autour de $10 \%{ }^{(18)}$. La décroissance des moins de 20 ans observée entre 1980 et 2008 se traduit quelque 30 ans plus tard par une diminution des effectifs adultes, diminution entretenant le rétrécissement de la base de la pyramide : moins de femmes en âge de procréer signifie moins de naissances, la plupart de ces pays ayant, de surcroît, vu la baisse de leur fécondité se poursuivre.

Par comparaison, dans la plupart des pays d'Europe de l'Ouest et du Nord, ces populations jeunes et adultes diminuent à peine (Autriche, Pays-Bas, Finlande, Danemark), stagnent (France, Belgique, Suisse) ou même augmentent (Royaume-Uni, Irlande, Luxembourg, Norvège, Suède).

\section{Forte croissance de la population très âgée dans les prochaines décennies}

Dans les années à venir, partout en Europe ce fort vieillissement par le haut de la pyramide se doublera d'un vieillissement interne de la population âgée. Autrement dit, les effectifs de 80 ans et plus vont augmenter beaucoup plus fortement que ceux de 65-79 ans (figure 31). À de rares exceptions près, la population la plus âgée va doubler d'ici à 2040 ; elle risque même de tripler dans certains pays (Chypre, Malte, Pologne, Irlande).

À l'échelle de l'ensemble de l'Europe, la proportion de personnes âgées de 80 ans et plus dans la population totale atteignait en 2008 près de $4 \%$. Dans la moitié des pays, cette proportion dépassait 3,7 \% et seuls 3 d'entre eux (Suède, Italie, France) avaient franchi le seuil de $5 \%$. En 2040, la part des très âgés pourrait être supérieure à $8 \%$ dans plus d'un pays sur deux, et même à $10 \%$ en Finlande, Allemagne et Italie (tableau annexe A.10).

(18) Sauf à Malte où la population âgée de moins de 20 ans diminue de plus de $21 \%$. 
Figure 31. Taux de croissance (\%) entre 2008 et 2040 des effectifs âgés de 65-79 ans et de 80 ans et plus

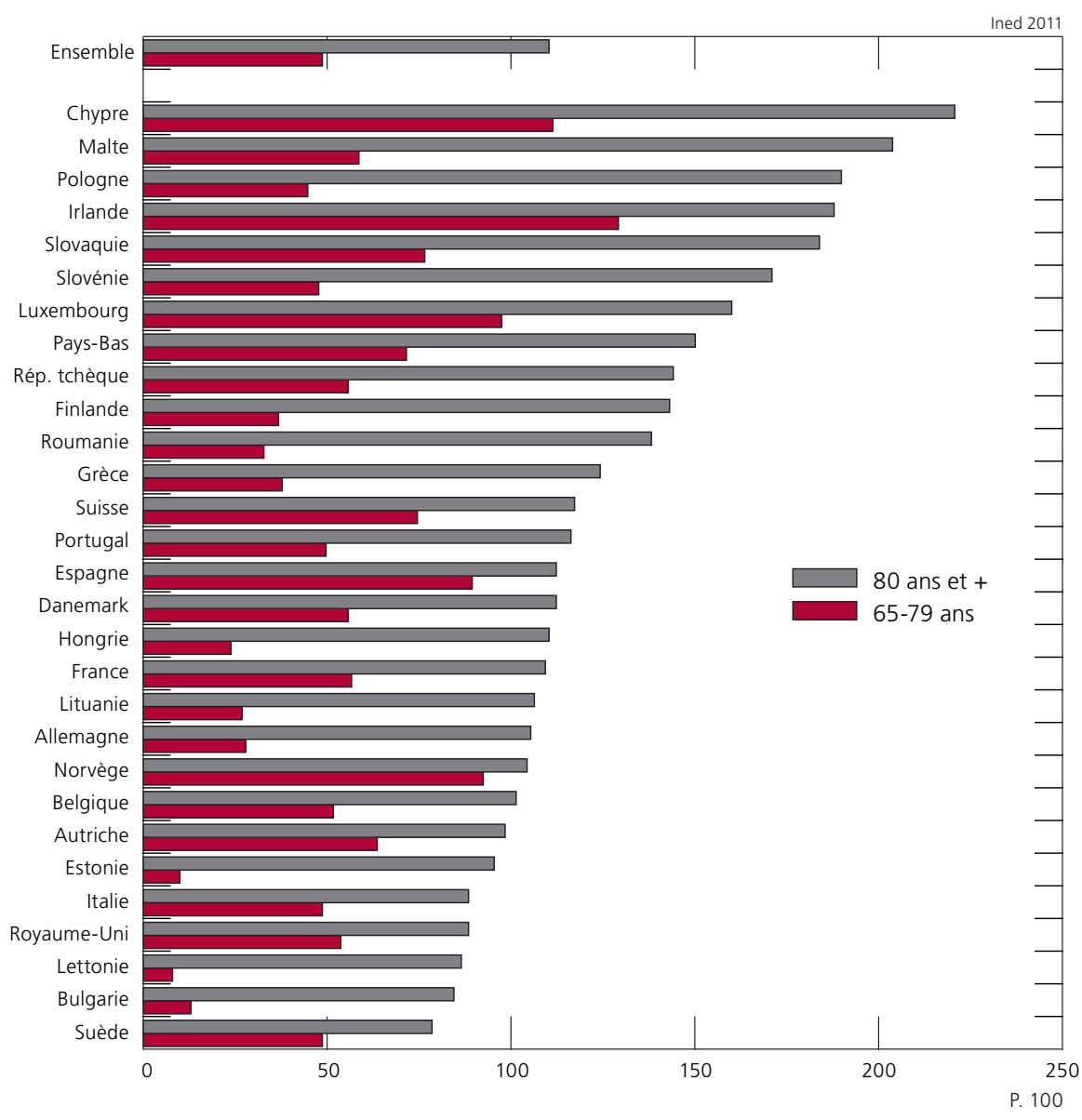

Source : Eurostat.

Or, même si la majorité des personnes âgées vieillissent sans incapacité et que les générations futures atteindront certainement les divers stades de la vieillesse en meilleure santé, le risque de dépendance, physique et psychique, augmente avec l'avance en âge, rendant souvent indispensable un soutien au quotidien. La forte progression des très âgés laisse donc présager un important accroissement des besoins d'aide et l'on sait que, partout en Europe, les conjoints jouent dans ce domaine un rôle essentiel. Or l'une des conséquences majeures de la baisse de la mortalité est qu'en différant l'entrée dans le veuvage, elle prolonge la vie en couple. Dans les prochaines décennies, cette tendance à la baisse $\mathrm{du}$ veuvage fera plus que contrebalancer la croissance du divorce, encore faible dans ces générations, et conduira plus souvent les personnes très âgées à vieillir à deux. Les femmes, en particulier, qui en raison de la surmortalité masculine sont plus souvent veuves, pourront plus fréquemment compter sur la présence 
d'un partenaire pour faire face à leur dépendance (40 \% en 2030 contre $21 \%$ en 2000 chez les 75-84 ans) et ceci dans tous les pays (Gaymu et al., 2007).

Toutefois, la croissance de la population masculine confrontée à la dépendance de leur conjointe ${ }^{(19)}$ et la plus grande survie de couples dont les deux membres souffrent d'incapacités laissent présager un surcroît de besoins d'ordre professionnel. Or partout en Europe, l'incertitude reste de rigueur sur le financement futur des retraites et, par là même, sur la capacité financière des aînés de demain à faire appel à des aides professionnelles pour les soutenir au quotidien. De plus, si dans de nombreux pays il y a eu, durant les dernières décennies, une réelle volonté politique d'assistance, devant l'ampleur du coût de la protection sociale, cette solidarité collective ne risque-t-elle pas de s'effriter ? La dégradation de la situation économique des personnes âgées et/ou la démission des pouvoirs publics en matière d'aide au maintien à domicile se traduira, ipso facto, par une pression supplémentaire sur les familles, et en particulier sur les femmes, alors même qu'elles assument déjà, aujourd'hui, l'essentiel de la solidarité à l'égard des plus fragiles (Mestheneos et Triantafillou, 2005).

\section{La transformation de l'Europe en un continent d'immigration}

De continent d'émigration jusqu'au milieu du $\mathrm{XX}^{\mathrm{e}}$ siècle, l'Europe s'est progressivement transformée en continent d'immigration au cours des 50 dernières années (partie II, figure 2D). Le recrutement de travailleurs étrangers durant la période de croissance économique de l'après-guerre, ainsi que les retours de ressortissants nationaux à la suite des décolonisations, caractérisent la première étape de cette évolution. La crise économique de 1974 marque la fin de cette période ; les flux migratoires à destination de l'Europe et entre pays européens ralentissent pendant près d'une décennie. L'instabilité politique et socioéconomique dans les anciens pays socialistes au sud-est et au centre de l'Europe et l'ouverture de leurs frontières à la fin des années 1980 expliquent l'accroissement des migrations au départ de ces pays à ce moment-là.

À l'échelle mondiale, on observe aussi une accélération des migrations à destination des pays développés provenant des pays en développement, en raison de la persistance des inégalités Nord/Sud (Nations unies, 2009a). Durant les deux décennies suivantes, les migrations atteindront des niveaux importants dans un nombre toujours croissant de pays.

Parallèlement, la contribution des migrations à la croissance démographique des pays européens augmente. Aujourd'hui, la majorité des États ont un solde migratoire supérieur au solde naturel, et les pays dont le solde naturel est élevé (France, Irlande) ou le solde migratoire négatif (Pologne) constituent des exceptions.

(19) Les hommes ont aujourd'hui plus de difficultés à assumer la dépendance de leur épouse que l'inverse : ils ont plus souvent recours à des aides professionnelles ou placent plus fréquemment leur conjointe en institution (Gaymu et al., 2006). 


\section{1. Évolution générale des soldes migratoires}

Le solde migratoire est un indicateur du volume des migrations entre un pays et le reste du monde, quelle qu'en soit la direction. Lorsque les gains migratoires sont supérieurs aux pertes (les entrées sur le territoire sont plus nombreuses que les sorties), le solde est positif; il peut aussi être négatif (sorties plus nombreuses que les entrées) ou fortuitement nul. La figure 32 illustre l'évolution des taux d'accroissement migratoire par région ${ }^{(20)}$ et pour l'ensemble de l'Europe depuis 1960, et la figure 33 les valeurs maximales et minimales des soldes migratoires enregistrés par les pays européens. Comme ce dernier indicateur est corrélé à la taille de la population, les pays les plus peuplés d'Europe y sont davantage représentés.

Figure 32. Taux d'accroissement migratoire par région depuis 1960

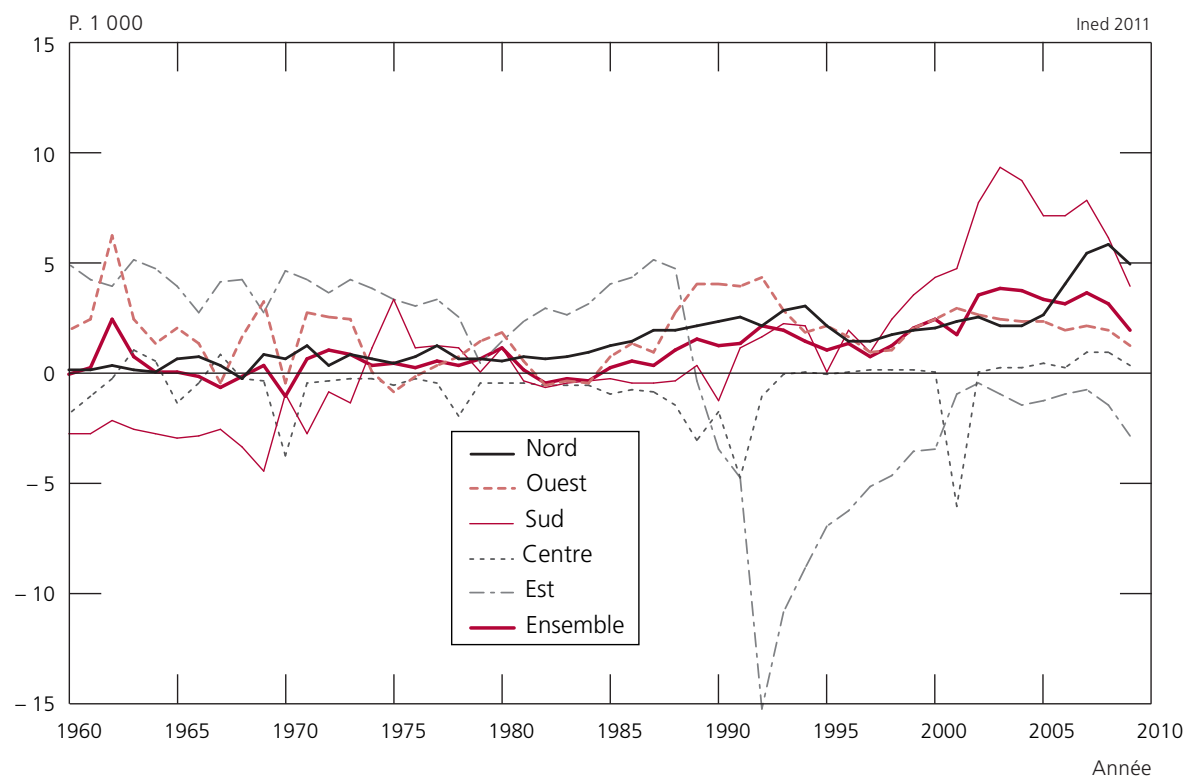

Sources : Base de données des pays développés (Ined) ; base de données Devision du Centre d'études démographiques (Moscou).

Le taux d'accroissement migratoire de l'ensemble de l'Europe oscille autour de $0 \%$ pendant la première moitié de la période étudiée (figure 32). Il ne devient définitivement positif qu’à partir du milieu des années 1980 et augmente progressivement ensuite. Cependant chaque région connaît une évolution spécifique. Avant 1974, l'accroissement migratoire est positif en Europe de l'Ouest et du Nord, qui accueillent alors la majorité des immigrants provenant

(20) Il s'agit ici de taux calculés pour chacune des 5 régions, et non de la moyenne arithmétique des taux de solde migratoire des pays composant la région, car les effectifs des populations sont parfois trop inégaux pour que la moyenne arithmétique des taux ait un sens. 
des autres régions d'Europe, mais aussi du monde. Entre 1960 et 1964, la France enregistre un solde positif de 1,6 million de personnes, ce nombre incluant aussi bien des migrants travailleurs que des rapatriés de l'Algérie devenue indépendante en 1962 (figure 33). L'Europe du Sud est une région de forte émigration : son taux d'accroissement migratoire est alors négatif, - 2,5\%o en moyenne de 1960 à 1974. Durant ces 15 ans, le déficit migratoire atteint 1,5 million d'habitants au Portugal, 1,2 million en Italie, et 0,7 million en Espagne. Les pays de l'Europe du Centre participent faiblement aux échanges migratoires et enregistrent un taux moyen de - 0,6\%o. Le milieu des années 1970 constitue une rupture à plusieurs titres : les migrations sont moins nombreuses dans la décennie suivante avant d'atteindre des niveaux historiques dans les années 1990 et 2000.

Figure 33. Soldes migratoires quinquennaux maximaux et minimaux en Europe depuis 1960

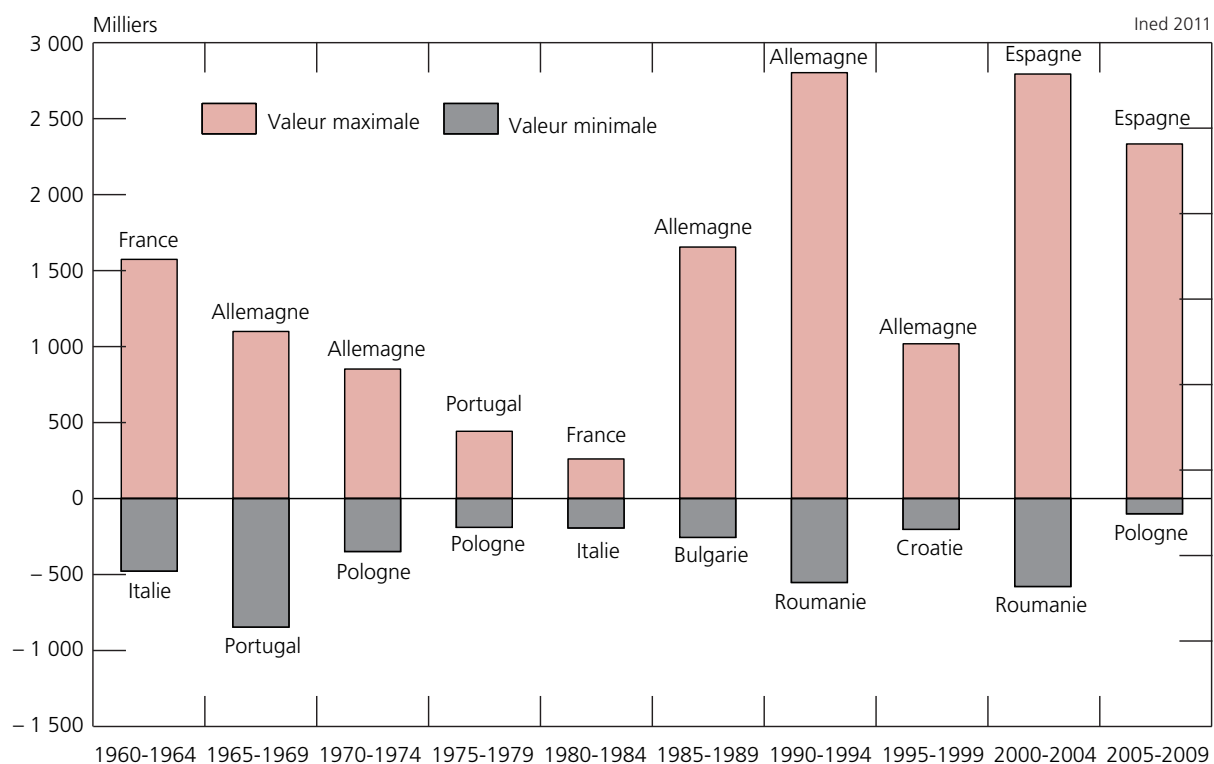

Lecture : En 1960-1964, I'Italie est le pays européen ayant enregistré la plus grande perte de population due aux migrations ( 480000$)$, la France celui enregistrant le plus grand gain de population (+ 1580000$)$ au cours de ces cinq années.

Sources : Base de données des pays développés (Ined) ; base de données Devision du Centre d'études démographiques (Moscou).

À la fin des années 1980, lorsque les migrations augmentent à nouveau, c'est l'Europe de l'Ouest qui continue d'enregistrer les taux d'accroissement migratoire les plus élevés dans un premier temps. L'Allemagne est alors la principale destination des migrants : son solde migratoire cumulé pour la période 1985-1999 s'élève à 5,5 millions, dont plus de la moitié enregistrée entre 1990 et 1994 (figure 33). Les pays d'Europe du Sud affichent des soldes 
migratoires positifs au début des années 1990, mais ce n'est qu'à partir des années 2000 qu'ils rivalisent avec les anciens pays d'immigration en termes de volume. De 2000 à 2004, la population espagnole s'accroît de 2,8 millions d'habitants du fait des migrations, et de 2,3 millions supplémentaires dans les cinq années suivantes. L'Italie affiche un solde de 3,8 millions durant la décennie 2000, et la Grèce et le Portugal d'environ 0,4 million chacun.

Le solde migratoire d'Europe centrale, où se situent des pays à forte émigration (Pologne, Roumanie), mais aussi d'autres qui attirent les immigrants (Hongrie, République tchèque), baisse sensiblement à la fin des années 1980, et depuis tend vers zéro. Cependant, c'est dans cette région (composée des anciens pays socialistes d'Europe du Centre et du Sud-Est) qu'on observe dorénavant les départs les plus nombreux : entre 1985 et 2010, l'Albanie, la Bulgarie et la Pologne auraient chacune perdu un demi-million de leurs ressortissants et la Roumanie 1,3 million.

Le cas des pays d'Europe de l'Est (ici les pays Baltes) doit être interprété à la lumière de leur histoire récente. Faisant partie de l'Union soviétique jusqu'en 1991, ils ont accueilli un nombre important de migrants d'autres républiques soviétiques. Au lendemain de la dissolution de l'URSS, nombre d'entre eux, devenus entre-temps des migrants internationaux, ont décidé de retourner dans leurs pays d'origine, expliquant ainsi le solde migratoire négatif observé à partir des années 1990 .

Les volumes et caractéristiques des migrations restent fortement déterminés par des traits spécifiques aux pays (situation socioéconomique, proximité géographique et liens historiques avec d'autres pays...), comme le montre la figure 34 à travers l'exemple de cinq pays européens.

Vers 1975, les situations migratoires varient considérablement selon les pays. Si l'Italie est le seul pays avec une histoire récente d'émigration massive (figure 32), le Royaume-Uni comme l'Autriche ont déjà enregistré des soldes migratoires négatifs par le passé, notamment du fait des départs de nationaux. La Suède, pays d'émigration au XIX ${ }^{e}$ siècle, est déjà une importante destination d'immigration, surtout pour ses voisins nordiques. La République tchèque, faisant alors partie du bloc de l'Est, connaît un solde migratoire légèrement positif.

Lorsque les migrations reprennent à la fin des années 1980, ce sont l'Autriche et la Suède qui enregistrent les taux d'accroissement migratoire les plus importants (de l'ordre de 5 \%o). Les pics au début des années 1990 en Autriche (9\%o) sont liés à l'arrivée des réfugiés de la guerre en ex-Yougoslavie, pays proche géographiquement. Au même moment, l'Italie voit son solde redevenir positif par l'accueil de nombreux migrants d'Europe de l'Est, dont un nombre important d'Albanais. Une économie relativement développée par rapport à celles de ses voisins épargne à la République tchèque une émigration massive et attire même des migrants, principalement originaires de la nouvelle Slovaquie. Après une stagnation à la fin des années 1990, on observe une augmentation généralisée des taux migratoires, particulièrement en Italie (10 \%o en 20032004) et en République tchèque (8\%o en 2007). 
Figure 34. Taux d'accroissement migratoire (\%o) depuis 1975 dans cinq pays de l'Union européenne (frontières actuelles)

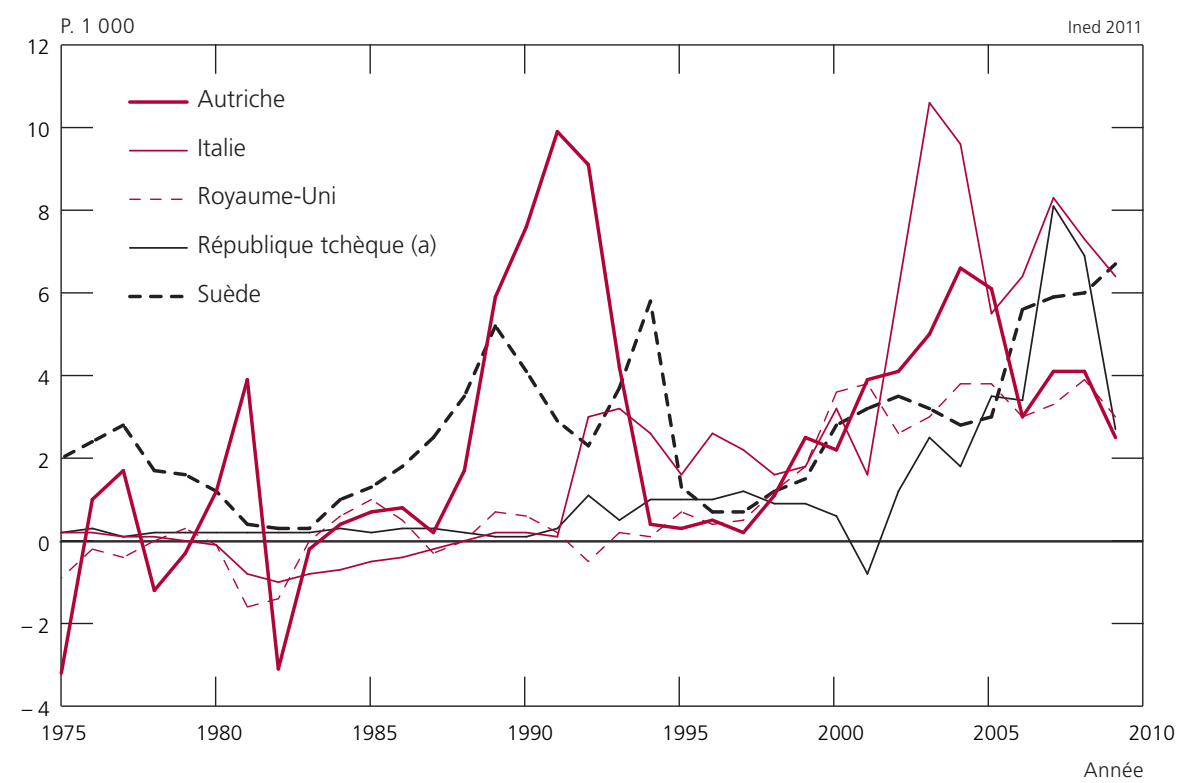

(a) La valeur négative en 2001 est due aux changements dans la procédure d'enregistrement des migrants plutôt qu'à une évolution réelle du solde migratoire.

Sources : Base de données des pays développés (Ined) ; base de données Devision du Centre d'études démographiques (Moscou).

Le Royaume-Uni, perçu comme un ancien pays d'immigration, connaît une situation plus complexe. Bien que destination importante pour son voisin le plus proche, l'Irlande, comme pour d'autres pays du Commonwealth, le Royaume-Uni enregistre des soldes migratoires nuls, voire négatifs jusqu'aux années 1990. Cela est dû à l'émigration régulière de ses ressortissants et à la mise en place d'une politique restrictive d'immigration à partir de la fin des années 1960. L'arrivée du parti travailliste en 1997, privilégiant une politique d'ouverture à certains immigrants sélectionnés, combinée au développement économique des années 1990 et 2000 (Somerville et al., 2009), expliquent l'augmentation du solde migratoire à partir du milieu des années 1990, dont le taux passe d'environ 0,5\%o à 3,5\%o dans les années 2000 .

Les mouvements migratoires en Europe s'expliquent par des facteurs internes (construction de l'UE, chute du mur...), mais aussi globaux. Une mise en perspective avec d'autres régions du monde, notamment des régions d'immigration, permet d'illustrer ce point (figure 35).

Les États-Unis ont été et demeurent un grand pays d'immigration : en 2010, un migrant sur cinq dans le monde y réside (Nations unies, 2009b). Les taux des États-Unis et de l'UE suivent une tendance similaire entre 1975 et 2000 : baisse de l'accroissement migratoire après 1974, augmentation à partir de la 
fin des années 1980 et relative stagnation au milieu des années 1990. Cependant, durant toute cette période les États-Unis accueillent plus d'immigrants, en effectifs comme en termes relatifs (le taux d'accroissement migratoire est en moyenne trois fois supérieur à celui de l'UE à 15). La situation semble s'inverser dans la dernière décennie. Aux États-Unis, le taux s'établit autour de 4,5\%o vers 2000 et baisse par la suite pour atteindre une moyenne de 3,3\%o en 20052010. Parallèlement, le taux augmente dans l'UE : il est multiplié par trois entre 1998 et 2003, année où il culmine à $5 \%$ o. On observe une baisse ces dernières années, qui coïncide avec la crise économique et financière de 2008.

Figure 35. Taux d'accroissement migratoire (\%o)

de I'Union européenne des 15, la Russie et des États-Unis depuis 1975

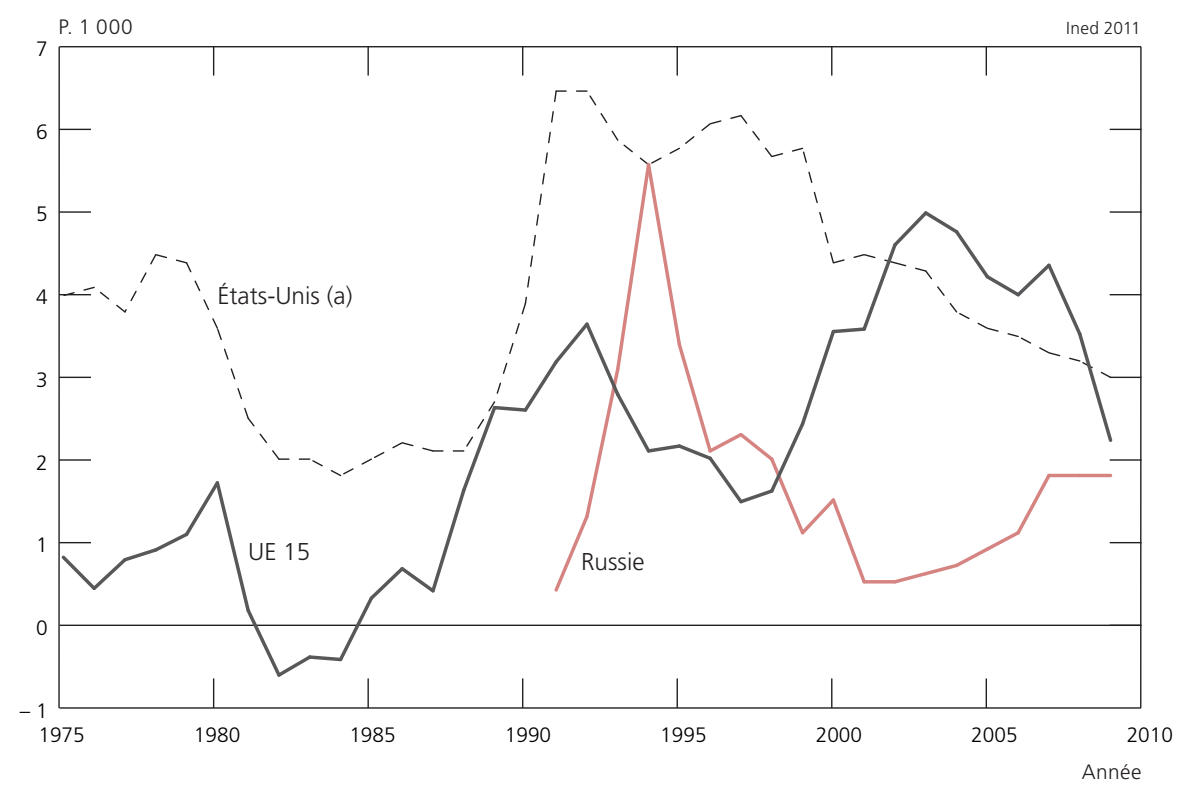

(a) En raison de l'absence de données, les niveaux pour la période 2005-2008 sont extrapolés à partir des tendances observées depuis 2000 et des estimations des Nations unies (2009c).

Sources: Base de données des pays développés (Ined) ; base de données Devision du Centre d'études démographiques (Moscou).

La situation migratoire de la Russie est liée à son histoire récente. Elle enregistre un solde migratoire positif depuis la dissolution de l'URSS en 1991, avec au départ une immigration comportant une forte composante ethnique (retour des ressortissants russes d'anciennes républiques d'URSS). Cette « réserve » démographique tendant à s'épuiser, la Russie accueille à présent de plus en plus de migrants non russes, venant également d'anciennes républiques, principalement d'Asie centrale. La Russie et l'UE suivent des tendances complètement opposées ces deux dernières décennies. Hormis les premières années après l'indépendance de la Russie, l'UE enregistre des soldes migratoires toujours plus élevés, bien que la crise ait rapproché leurs niveaux, qui avoisinent à présent $2 \%$. 
Au début de la période étudiée, la situation migratoire en Europe variait fortement selon ses régions. Même si des différences persistent et si certains pays continuent d'être des zones de forte émigration, la population européenne augmente globalement grâce aux migrations. Elle est aujourd'hui une destination aussi importante pour les migrants internationaux que les pays d'émigration plus « anciens » (États-Unis, Canada, Australie).

\section{Flux migratoires et caractéristiques des migrants depuis 1990}

\section{En majorité des entrées de personnes nées à l'étranger et des sorties de natifs}

Bien que le solde migratoire soit défini comme la différence observée durant une période entre le nombre d'entrées et de sorties d'un territoire, seuls les pays ayant des registres de population comptabilisant l'ensemble des mouvements sur leur territoire peuvent le calculer directement de cette manière. Les autres pays estiment le solde migratoire indirectement au moment d'un recensement, par la différence entre la variation totale de la population et le solde naturel entre deux recensements (on parle alors de solde migratoire « apparent »). Le niveau du solde migratoire ainsi obtenu est moins fiable car il ne dépend pas seulement des mouvements migratoires, mais aussi de la qualité respective des deux recensements et de l'enregistrement des naissances et des décès. Par ailleurs, ce mode de calcul ne permet pas de connaître la composition du solde : un solde nul peut signifier une absence de mouvements ou une stricte compensation des entrées par les sorties.

Les Nations unies recommandent aux pays de décomposer les flux selon le pays de naissance et/ou la nationalité des migrants (Nations unies, 1998). La première caractéristique est permanente (le lieu de naissance d'une personne ne change pas, même lorsqu'elle migre), alors que la deuxième peut changer une ou plusieurs fois dans la vie d'un individu (suite à l'acquisition de la nationalité du pays d'accueil). Historiquement, les statistiques selon le critère de la nationalité sont les plus répandues, même si certains pays ont commencé à produire des statistiques par lieu de naissance ${ }^{(21)}$. Bien que les deux variables soient très liées, il arrive que les entrées de nationaux correspondent à des entrées de personnes nées à l'étranger et arrivant pour la première fois dans le pays. C'est le cas des minorités ethniques allemandes installées dans les pays d'Europe de l'Est depuis plusieurs générations, ou plus récemment des descendants d'émigrés espagnols ou italiens en Amérique latine, bénéficiant du droit du sang et se réinstallant dans le pays d'origine de leurs parents (Padilla et Peixoto, 2007).

(21) Le règlement (CE) n 862/2007 du Parlement européen et du Conseil du 11 juillet 2007 relatif aux statistiques communautaires sur la migration et la protection internationale, prévoit le double classement des entrées/sorties selon la nationalité et le lieu de naissance des migrants. Ces statistiques sont collectées par Eurostat selon la nationalité depuis 1998, et selon le lieu de naissance depuis 2008. 
Les figures 36A et 36B montrent le nombre annuel d'immigrations et d'émigrations selon le lieu de naissance des migrants, ainsi que le solde migratoire en résultant aux Pays-Bas depuis 1995. Sur ces 15 dernières années, le nombre de sorties de natifs des Pays-Bas est nettement supérieur au nombre d'entrées, tandis que la situation est inverse pour les personnes nées à l'étranger, conduisant à un solde migratoire négatif pour les premiers et positif pour les seconds. À l'échelle de la population entière, le solde migratoire est positif, hormis en 2003-2007 lorsque l'augmentation des sorties dans les deux groupes de migrants a été accompagnée d'une baisse substantielle des entrées de personnes nées à l'étranger. Ces flux réagissent différemment aux changements de contexte, comme le montre la période récente marquée par la crise économique. Le nombre de départs des natifs des Pays-Bas a baissé de $20 \%$ entre 2008 et 2009, celui des personnes nées à l'étranger a augmenté de $8 \%$ tandis que le nombre de leurs entrées, en croissance jusque-là, a plafonné.

Figure 36. Effectif annuel d'immigrants et d'émigrants $(A)$ et solde migratoire annuel (B) aux Pays-Bas depuis 1995 selon le lieu de naissance des migrants

A. Effectif annuel d'immigrants et d'émigrants

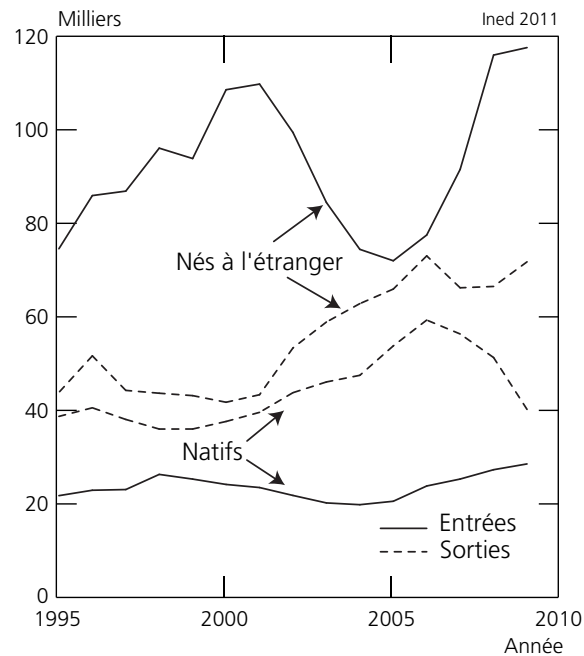

B. Solde migratoire annuel

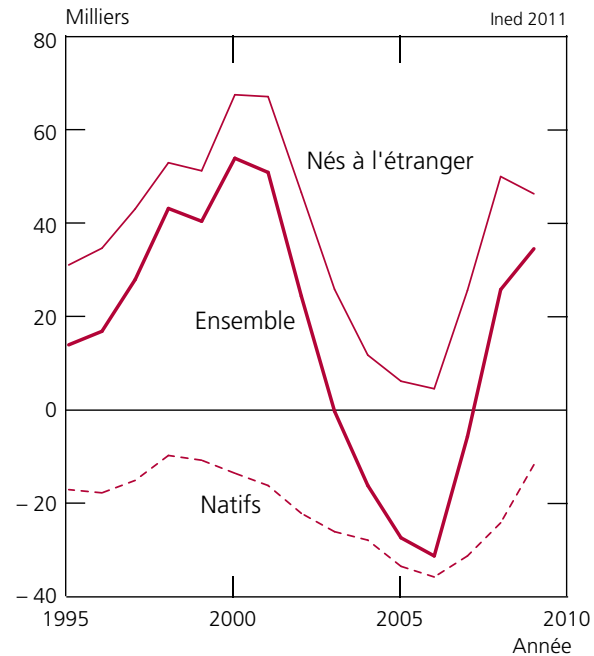

Source : Statistics Netherlands.

Le solde migratoire est souvent associé aux mouvements d'individus nés à l'étranger ; l'exemple des Pays-Bas montre que les mouvements de natifs ont aussi un rôle dans son évolution. Le tableau 9 décompose les différents types de flux - entrées et sorties de natifs et de personnes nées à l'étranger - dans le volume total des migrations ${ }^{(22)}$ de quelques pays européens en 2008.

(22) Le volume total des migrations est la somme de quatre flux : les entrées des natifs du pays, les sorties des natifs du pays, les entrées des personnes nées à l'étranger et les sorties des personnes nées à l'étranger. 
Tableau 9. Répartition (\%) du volume total des migrations de quelques pays européens en 2008 selon les quatre types de flux

\begin{tabular}{|c|c|c|c|c|c|c|}
\hline \multirow[b]{2}{*}{ Région } & \multirow[b]{2}{*}{ Pays } & \multicolumn{2}{|c|}{ Immigration } & \multicolumn{2}{|c|}{ Émigration } & \multirow[b]{2}{*}{ Total } \\
\hline & & Natif & $\begin{array}{c}\text { Né à } \\
\text { l'étranger }\end{array}$ & Natif & $\begin{array}{c}\text { Né à } \\
\text { l'étranger }\end{array}$ & \\
\hline \multirow[t]{3}{*}{ Nord } & Danemark & 16,3 & 43,7 & 13,3 & 26,8 & 100 \\
\hline & Finlande & 18,2 & 49,5 & 20,1 & 12,2 & 100 \\
\hline & Suède & 9,1 & 59,9 & 14,1 & 16,8 & 100 \\
\hline \multirow[t]{4}{*}{ Ouest } & Autriche & 7,0 & 52,3 & 9,7 & 31,0 & 100 \\
\hline & Irlande & 15,8 & 37,0 & 19,9 & 27,3 & 100 \\
\hline & Pays-Bas & 11,6 & 49,9 & 19,7 & 18,8 & 100 \\
\hline & Royaume-Uni & 7,0 & 51,0 & 17,0 & 25,0 & 100 \\
\hline \multirow[t]{3}{*}{$\operatorname{Sud}^{(a)}$} & Espagne & 2,4 & 70,7 & 3,3 & 23,5 & 100 \\
\hline & Italie & 4,4 & 82,4 & 6,5 & 6,6 & 100 \\
\hline & Slovénie & 5,7 & 66,0 & 6,6 & 21,7 & 100 \\
\hline Centre $^{(a)}$ & Hongrie & 0,1 & 88,5 & 1,3 & 10,1 & 100 \\
\hline \multirow[t]{2}{*}{ Est } & Estonie & 14,9 & 30,2 & 44,7 & 10,3 & 100 \\
\hline & Lituanie & 21,0 & 14,4 & 45,8 & 18,8 & 100 \\
\hline
\end{tabular}

Les entrées des personnes nées à l'étranger constituent le flux le plus important dans tous les pays, à l'exception de l'Estonie et de la Lituanie, et représentent entre $37 \%$ et $89 \%$ de l'ensemble des mouvements. Mais les départs des natifs du pays représentent aussi une proportion non négligeable dans les pays d'Europe du Nord et de l'Ouest, allant de $10 \%$ en Autriche jusqu'à $20 \%$ en Finlande, en Irlande et aux Pays-Bas. Ainsi l'Irlande, vue ces dernières années comme un pays d'immigration, n'a pas complètement rompu avec sa tradition d'émigration, revivifiée par la crise économique récente.

\section{Des immigrants jeunes et d'origines variées}

Pour dresser un portrait des immigrants d'origine étrangère, nous utilisons les données par nationalité (et non par lieu de naissance) compte tenu de leur plus grande disponibilité. Le tableau 10 présente les dix pays ayant reçu le plus d'immigrants de nationalité étrangère en 1998 et 2008. Alors que les principales destinations n'ont pas changé durant cette décennie, le nombre d'immigrants accueillis et l'ordre des destinations ont évolué. Le nombre d'entrées d'étrangers dans ces pays a doublé en 10 ans (de 1,4 million en 1998 à 2,9 millions en 2008), l'Espagne contribuant à $40 \%$ de cette augmentation. À l'exception de l'Allemagne, cette augmentation a été générale et le nombre de pays accueillant plus de 100000 immigrants est passé de quatre en 1998 à sept en 2008. L'Allemagne, au premier rang en 1998 avec plus de 600000 nouveaux immigrants, est désormais dépassée par l'Espagne, avec près de 700000 entrées en 2008. 
Tableau 10. Nombre d'immigrants étrangers arrivés en 1998 et 2008 dans les dix principaux pays de destination en Europe

\begin{tabular}{|c|c|c|c|}
\hline Pays & 1998 & 2008 & $\begin{array}{c}\text { Coefficient } \\
\text { multiplicateur }\end{array}$ \\
\hline Espagne & 57195 & 692228 & 12,1 \\
\hline Allemagne & 605500 & 573815 & 0,9 \\
\hline Italie & 127114 & 496549 & 3,9 \\
\hline Royaume-Uni & 238503 & 455290 & 1,9 \\
\hline France $^{(a)}$ & 155879 & 211055 & 1,4 \\
\hline Suisse & 72202 & 161629 & 2,2 \\
\hline Belgique & 59666 & 109926 & 1,8 \\
\hline Autriche & 59229 & 94376 & 1,6 \\
\hline Pays-Bas & 81701 & 94335 & 1,2 \\
\hline Suède & 35701 & 82972 & 2,3 \\
\hline \multicolumn{4}{|c|}{$\begin{array}{l}\text { (a) À partir de 2004, les ressortissants de l'Union européenne n'ont plus obligation de posséder un titre de séjour } \\
\text { en France et ne figurent plus dans les statistiques d'immigration officielles. Le nombre d'immigrants issus de ces } \\
\text { pays en } 2008 \text { est estimé. } \\
\text { Sources : Eurostat (migr_imm2ctz) ; Ined, Flux d'immigration, } \\
\text { http://www.ined.fr/fr/pop_chiffres/france/flux_immigration/ }\end{array}$} \\
\hline
\end{tabular}

Les pays d'origine des immigrants ont aussi évolué. Avant 1974, la provenance des migrants était déterminée en grande partie par des liens historiques, notamment de colonisation, et la signature de traités bilatéraux recrutant des travailleurs étrangers. À présent, nous observons une diversification des origines due à une généralisation des migrations internationales; de nouvelles filières s'établissent, au départ de nouveaux pays d'émigration, mais aussi d'anciens pays d'émigration investissant de nouvelles destinations. Le tableau 11 présente les cinq nationalités les plus représentées dans plusieurs pays européens et leur poids parmi l'ensemble des immigrants étrangers en 1998 et 2008.

Parmi ces pays, la provenance des immigrants principaux n'est restée identique qu'en Suisse et en Hongrie, et a peu changé en République tchèque. Dans les autres pays, on observe plusieurs évolutions. Les communautés avec la plus longue présence en Europe de l'Ouest (Maroc, Turquie) sont depuis quelques années numériquement dépassées par les nouveaux pays membres de l'UE (Bulgarie, Hongrie, Pologne, Roumanie). Les migrants d'Albanie, d'ex-Yougoslavie, de Russie ou d'Ukraine constituent une proportion importante des flux à destination de plusieurs pays de l'Europe du Sud et du Centre.

Parmi les nationalités asiatiques, seuls les flux des ressortissants chinois et philippins sont significatifs dans plusieurs pays. La présence des Vietnamiens en République tchèque est plus ancienne et circonscrite à ce pays ; elle est due aux accords d'échanges signés à l'époque du régime communiste. Le RoyaumeUni se distingue des autres pays par l'importance des communautés asiatiques dans ses flux. Les originaires du continent latino-américain sont surtout présents, et de plus en plus, en Espagne. La Suisse, comme le Luxembourg, se caractérisent par la forte proportion de migrants originaires de l'UE des 15. Les pays d'Europe 


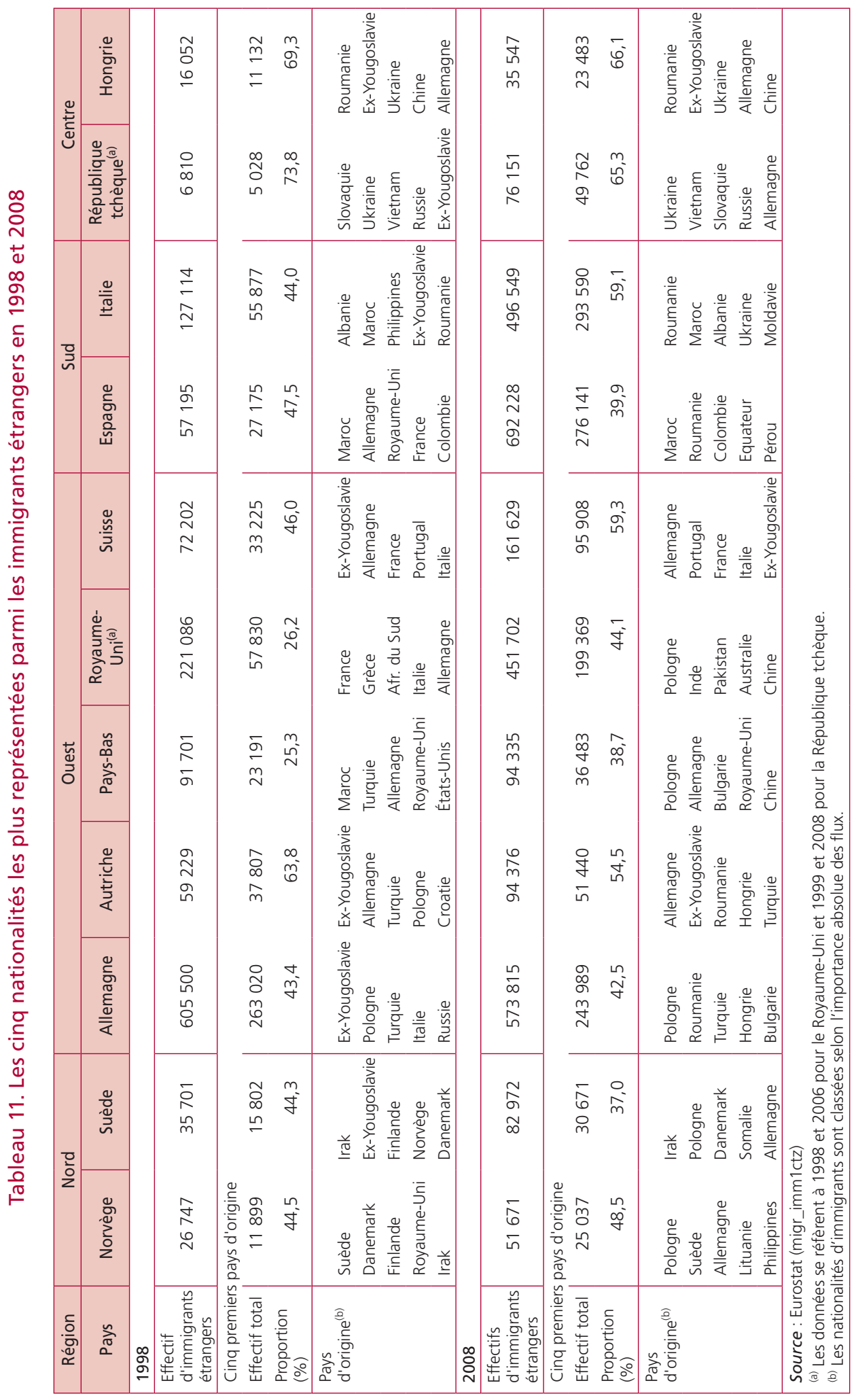


du Nord, accueillant jusque récemment des ressortissants majoritairement nordiques, voient les origines de leurs immigrants se diversifier avec l'arrivée des réfugiés d'Irak ou de Somalie, mais aussi des migrants de nouveaux pays de l'UE. Parmi les pays d'Europe de l'Ouest, seule l'Allemagne voit émigrer un nombre substantiel de ses ressortissants (175 000 émigrations en 2008) et figure en tête des immigrants arrivés dans huit pays.

Une telle analyse ne peut être réalisée pour la France, faute de données adéquates : à partir de 2004, les ressortissants de l'Union européenne, n'ayant plus obligation de posséder un titre de séjour en France, ne figurent plus dans les statistiques officielles d'immigration qui sont établies à partir des nouveaux titres de séjours délivrés. Si l'on se limite aux cinq premières nationalités parmi les pays tiers (hors UE), elles sont restées identiques pendant cette décennie: Algérie, Maroc, Chine, Tunisie et Turquie ; leur proportion parmi l'ensemble des immigrants des pays tiers est passée de $30 \%$ à $36 \%$. Cependant en 1998, l'Allemagne, le Royaume-Uni, le Portugal et l'Italie comptabilisaient chacun entre 5000 et 8000 entrées, se situant alors au même niveau d'entrées que la Tunisie, la Turquie et la Chine (Ined, Flux d'immigration).

Les immigrants sont majoritairement des hommes, mais certains pays font exception (tableau 12). Les proportions de femmes les plus faibles s'observent en Europe de l'Est (entre $20 \%$ et $40 \%$ ), à l'inverse des pays d'Europe du Sud qui accueillent une majorité de femmes (autour de $53 \%$ ). Entre ces deux extrêmes nous retrouvons la plupart des pays d'Europe de l'Ouest, où la proportion de femmes oscille entre $45 \%$ et $49 \%$. L'Allemagne constitue à ce titre une exception, ayant toujours accueilli une proportion assez faible de femmes (environ $40 \%)^{(23)}$. À l'inverse, la France se distingue par le poids important des migrations féminines (54\% de l'ensemble).

Tableau 12. Proportion (\%) de femmes parmi les immigrants étrangers en 2008

\begin{tabular}{|c|c|c|c|c|c|c|c|}
\hline \multicolumn{2}{|c|}{ Moins de $40 \%$} & \multicolumn{2}{|c|}{$40 \%$ à $45 \%$} & \multicolumn{2}{|c|}{$45 \%$ à $50 \%$} & \multicolumn{2}{|c|}{$50 \%$ et plus } \\
\hline Slovénie & 20,5 & Allemagne & 40,0 & Suisse & 45,7 & Portugal & 50,9 \\
\hline Slovaquie & 30,9 & Estonie & 40,0 & Danemark & 47,6 & Irlande & 53,1 \\
\hline Lettonie & 36,5 & Hongrie & 41,0 & Belgique $^{(a)}$ & 47,9 & Espagne & 53,5 \\
\hline \multirow[t]{3}{*}{ Rép. tchèque } & 36,7 & Norvège & 43,4 & Autriche & 48,0 & France & 53,6 \\
\hline & & Royaume-Uni ${ }^{(a)}$ & 44,9 & Suède & 48,1 & Italie & 54,1 \\
\hline & & & & Pays-Bas & 48,6 & & \\
\hline
\end{tabular}

(23) Cela résulte, entre autres, de la politique d'immigration conduite après la guerre en Allemagne. N'ayant pas d'anciennes colonies, la majorité de travailleurs étrangers (gastarbeiter) ont été recrutés par l'intermédiaire d'accords bilatéraux signés entre l'Allemagne et les autres pays (Italie, Grèce, Turquie, Portugal, Yougoslavie). Ces contrats étaient souvent de courte durée et concernaient principalement des emplois masculins. 
La part des femmes dans les flux d'immigrants ne suit pas une évolution univoque dans le temps, ce qui va à l'encontre de l'idée généralement répandue d'une féminisation des migrations, faisant suite aux restrictions de l'immigration de travail depuis les années 1970. L’analyse détaillée des statistiques d'immigration et d'émigration dans quatre pays (Belgique, Allemagne, RoyaumeUni et États-Unis) montre que même avant 1974, certains flux comprenaient une proportion importante, voire une majorité de femmes, notamment parmi les immigrants nationaux ou ceux originaires de pays développés (Zlotnik, 1995). Après 1974, la proportion de femmes a certes augmenté dans certains groupes (les immigrants d'Amérique latine et d'Asie du Sud-Est, les retours de minorités ethniques en Allemagne), mais dans l'ensemble les flux d'immigrants sont restés majoritairement masculins. Une féminisation des flux migratoires n'est perceptible que dans l'évolution du solde migratoire. Étant donné que l'on enregistre généralement plus de départs d'hommes que de femmes, cet indicateur synthétique des entrées et des sorties donne un poids plus important aux femmes dans son évolution.

La structure par âge des immigrants est généralement plus jeune que celle de la population qu'ils rejoignent ${ }^{(24)}$. Il y a une forte concentration aux âges adultes (15-64 ans) : selon les destinations, ce groupe représente entre $70 \%$ et $90 \%$ des immigrants. En 2008, les immigrants les plus jeunes (27-28 ans en moyenne) arrivent dans les pays d'Europe du Nord, tandis que les immigrants plus âgés (32-36 ans) sont en Europe centrale et orientale (figure 37). Les

Figure 37. Âge moyen des immigrants étrangers en 2008 selon le sexe et le pays d'accueil

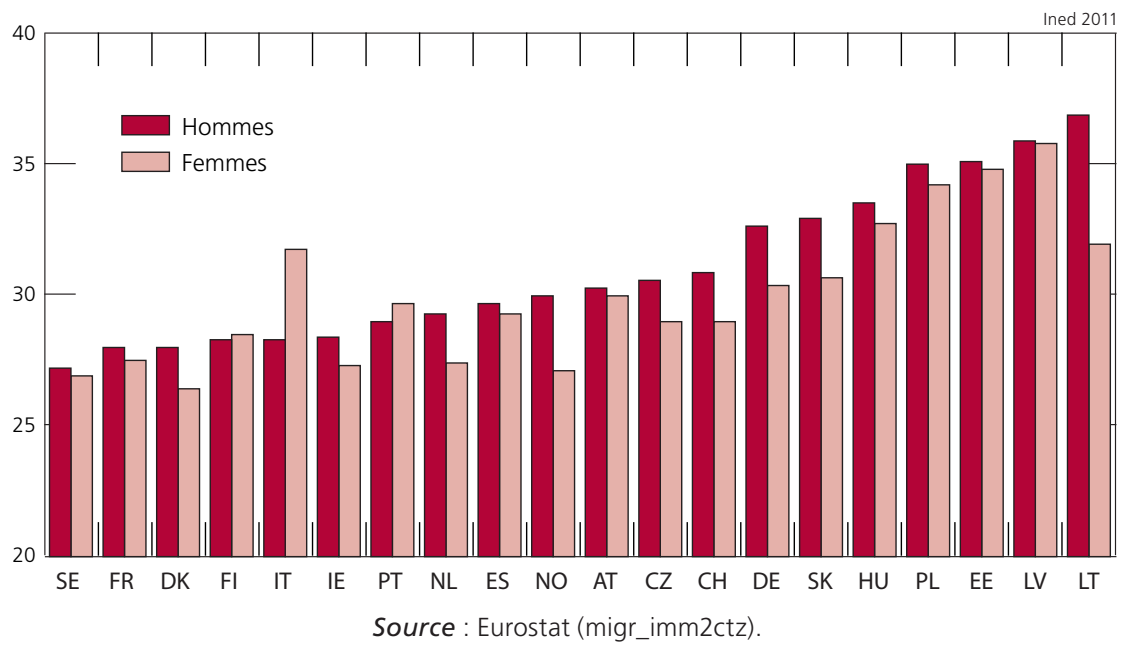

(24) Les immigrants nationaux sont généralement plus âgés que les immigrants étrangers, surtout dans les pays comptant un nombre important de départs de nationaux (Autriche, Pays-Bas, Finlande), qui retournent le plus souvent dans leur pays d'origine après avoir passé quelques années à l'étranger. 
femmes sont généralement de 1 à 2 ans plus jeunes en moyenne que les hommes, à l'exception des immigrantes en Italie et au Portugal. Lâge élevé des immigrantes en Italie pourrait s'expliquer par la proportion importante de femmes déjà mariées (ayant ou non des enfants), venues des Philippines, d'Amérique du Sud ou d'Europe orientale pour travailler comme employée de maison.

\section{Diversité des motifs de la migration}

Les raisons poussant à la migration sont intéressantes, mais complexes à étudier. La dimension légale - le motif de délivrance d'un permis de séjour à un étranger ${ }^{(25)}$ - est le plus souvent utilisée pour les caractériser. Mais cette information n'est disponible que pour les migrants soumis à l'obligation d'un permis pour séjourner dans le pays; en sont donc exclus les ressortissants de l'UE ou de l'EEE bénéficiant de la libre circulation. Par ailleurs les nomenclatures des motifs de délivrance des titres dépendent étroitement des législations nationales en matière d'immigration et sont peu comparables entre différents pays ${ }^{(26)}$.

La migration familiale est souvent le premier motif de délivrance (tableau 13). Elle s'est même renforcée entre 2000 et 2005, sauf au Danemark qui a introduit des critères de regroupement familial plus stricts en 2002. Les études viennent en première position au Danemark, en deuxième position en France et aux Pays-Bas où elles représentent respectivement 33\%, $25 \%$ et $16 \%$ de l'ensemble des premiers titres délivrés en 2005. Les entrées à titre humanitaire étaient plus nombreuses au début des années 2000, mais restent importantes en Norvège et Suède (20\% et $17 \%$ de l'ensemble en 2005). À l'exception de l'Autriche, les titres délivrés pour motif de travail sont minoritaires : en France ils ne représentent que $6 \%$ en 2005, contre $10 \%$ en 2000.

Les autres motifs de la délivrance peuvent regrouper des profils de migrants très variés.

Les migrations de retraite sont mal couvertes par les statistiques courantes et ne peuvent être estimées qu'indirectement. Comme de nombreux retraités passent une partie de l'année dans leur pays d'origine et une autre dans celui d'accueil, ils ne remplissent pas les conditions pour être comptabilisés comme migrants internationaux dans les statistiques de flux, ni dans le recensement ; par conséquent, les deux sources sous-estiment leur nombre. Plusieurs facteurs sont à l'origine du développement de ce phénomène à partir des années 1980 : augmentation de la longévité, meilleure santé aux âges élevés, élévation du niveau de revenu, facilités d'installation au sein de l'UE, création de la zone euro facilitant le transfert des retraites, développement du tourisme et changements dans les attitudes et les préférences de style de vie (héliotropisme) (Gustafson, 2008).

(25) Dans quelques pays (Royaume-Uni, Chypre), les personnes sont interrogées sur les raisons de leur arrivée lors d'une enquête aux frontières.

(26) L'OCDE a commencé à rassembler des informations comparables pour les titres permanents (OCDE, 2008). Suite au règlement (CE) n $n^{\circ} 862 / 2007$ du Parlement européen et du Conseil du 11 juillet 2007 relatif aux statistiques communautaires sur la migration et la protection internationale, Eurostat collecte également ce type de données depuis 2008. 
Tableau 13. Répartition (\%) des premiers titres de séjour selon le motif de délivrance en 2000 et 2005

\begin{tabular}{|c|c|c|c|c|c|c|}
\hline $\begin{array}{c}\text { Motif de } \\
\text { délivrance }^{(a)}\end{array}$ & Autriche $\mathrm{e}^{(\mathrm{b})}$ & Danemark & France & Norvège & Pays-Bas ${ }^{(b)}$ & Suède \\
\hline \multicolumn{7}{|l|}{2000} \\
\hline Famille & 36,1 & 37,9 & 42,0 & 41,5 & 39,1 & 53,6 \\
\hline Travail & 51,5 & 12,3 & 9,9 & 2,0 & 9,7 & 9,8 \\
\hline Études & 8,6 & 19,3 & 30,1 & 9,9 & 7,0 & 3,8 \\
\hline Humanitaire & 2,2 & 19,5 & 4,3 & 46,5 & 39,2 & 27,2 \\
\hline Autres & 1,6 & 10,9 & 13,8 & 0,1 & 5,0 & 5,7 \\
\hline Total & 100,0 & 100,0 & 100,0 & 100,0 & 100,0 & 100,0 \\
\hline $\begin{array}{l}\text { Effectif } \\
\text { d'immigrants } \\
\text { étrangers }\end{array}$ & 65966 & 26406 & 149982 & 15326 & 69772 & 33789 \\
\hline \multicolumn{7}{|l|}{2005} \\
\hline Famille & 55,7 & 20,2 & 49,5 & 45,9 & 49,9 & 54,4 \\
\hline Travail & 32,5 & 17,1 & 5,9 & 21,0 & 15,1 & 12,6 \\
\hline Études & 8,9 & 32,6 & 24,7 & 12,6 & 15,8 & 9,4 \\
\hline Humanitaire & 1,6 & 4,5 & 8,1 & 20,3 & 6,1 & 165,0 \\
\hline Autres & 1,3 & 25,7 & 11,8 & 0,1 & 13,0 & 72,0 \\
\hline Total & 100,0 & 100,0 & 100,0 & 100,0 & 100,0 & 100,0 \\
\hline $\begin{array}{l}\text { Effectif } \\
\text { d'immigrants } \\
\text { étrangers }\end{array}$ & 53366 & 25553 & 187134 & 19209 & 48349 & 41541 \\
\hline \multicolumn{7}{|c|}{$\begin{array}{l}\text { (a) La classification résulte des présentations officielles en France, en Norvège, aux Pays-Bas et en Suède ; elle a } \\
\text { été élaborée par l'auteur pour l'Autriche et le Danemark. } \\
\text { (b) Les données se réfèrent aux années } 2002 \text { et } 2005 \text { pour l'Autriche, } 2000 \text { et } 2004 \text { pour les Pays-Bas. II n'existe } \\
\text { pas d'étude comparative portant sur les années plus récentes. } \\
\text { Source : Thierry (2008, p. } 73 \text {, tableau 3). }\end{array}$} \\
\hline
\end{tabular}

Les pays méditerranéens (Espagne, France, Italie, Malte, Portugal) accueillent principalement des retraités d'Europe du Nord et de l'Ouest (Allemagne, Danemark, Norvège, Pays-Bas, Royaume-Uni, Suède). En Espagne, les résidants ressortissants des pays de l'UE sont surreprésentés au-delà de 65 ans : ils représentent $74 \%$ des étrangers de cette classe d'âge, tandis qu'ils ne sont que 39 \% parmi les moins de 65 ans (OPI, 2008, tableau I.4). À l'intérieur de ce groupe, les ressortissants des nouveaux et des anciens pays membres de l'UE ont différentes caractéristiques : $27 \%$ des Britanniques et $18 \%$ des Allemands résidant en Espagne ont 65 ans ou plus contre moins de $1 \%$ des Bulgares et des Roumains. Ces derniers vivent plus souvent dans les grandes métropoles pour y travailler, tandis que les Britanniques et les Allemands se retrouvent plus souvent dans les stations balnéaires (OPI, 2009).

La France est une autre destination importante pour les retraités des pays d'Europe du Nord, principalement du Royaume-Uni et des Pays-Bas. En 2003, les Britanniques, avec 10800 entrées, figurent en troisième position par le nombre d'admissions au séjour, juste après l'Algérie et le Maroc (Ined, 2003). 
Ils sont plus âgés que les autres immigrants (43\% ont 50 ans ou plus contre $9 \%$ pour l'ensemble des immigrants) et détiennent un titre de séjour d' " inactif» dans la moitié des cas. Les ressortissants néerlandais, bien que moins nombreux (2 100 entrées en 2003), possèdent les mêmes caractéristiques : un tiers d'entre eux ont 50 ans ou plus et ils détiennent souvent le titre de séjour d' « inactif».

Même si, à l'heure actuelle, ces migrations sont moins importantes que celles de travail ou de famille, elles pourraient se développer rapidement dans les années à venir. Encore peu perceptibles dans les structures de population des pays de départ, les effets de ces migrations modifieraient alors le haut des pyramides des âges des pays peu peuplés ayant un niveau de vie élevé, comme le Danemark ou la Finlande (King et al., 1998).

\section{Les personnes nées à l'étranger quittent le pays d'accueil plus souvent que les natifs}

L'étude des mouvements migratoires s'arrête souvent à l'étape de l'immigration. Cet « oubli » des émigrations résulte de plusieurs facteurs, dont la difficulté à obtenir des informations n'est pas la moindre. Elles sont plus complexes à mesurer que les immigrations, notamment parce qu'un départ fait sortir l'individu du territoire national sur lequel s'effectue la collecte de statistiques ${ }^{(27)}$.

Tableau 14. Taux d'émigration (pour 1000 habitants) en 2008 selon le sexe et lieu de naissance

\begin{tabular}{|c|c|c|c|c|c|c|c|}
\hline \multirow{2}{*}{ Région } & \multirow{2}{*}{ Pays } & \multicolumn{3}{|c|}{ Natif du pays } & \multicolumn{3}{|c|}{ Né à l'étranger } \\
\hline & & Ensemble & Homme & Femme & Ensemble & Homme & Femme \\
\hline \multirow[t]{4}{*}{ Nord } & Danemark & 3 & 3 & 2 & 52 & 56 & 49 \\
\hline & Finlande & 2 & 2 & 2 & 24 & 28 & 20 \\
\hline & Norvège & 1 & 1 & 1 & 14 & 15 & 13 \\
\hline & Suède & 3 & 3 & 2 & 19 & 23 & 16 \\
\hline \multirow[t]{4}{*}{ Ouest } & Autriche & 3 & 3 & 2 & 45 & 55 & 36 \\
\hline & Irlande & 6 & 8 & 5 & 53 & 61 & 44 \\
\hline & Pays-Bas & 3 & 3 & 3 & 25 & 26 & 23 \\
\hline & Royaume-Uni & 3 & - & - & 38 & - & - \\
\hline \multirow[t]{2}{*}{ Sud } & Espagne & 1 & 1 & 1 & 37 & 29 & 45 \\
\hline & Italie & 1 & 1 & 1 & 9 & 10 & 9 \\
\hline Centre & Hongrie & 0 & 0 & 0 & 10 & 14 & 6 \\
\hline \multirow[t]{2}{*}{ Est } & Estonie & 3 & 3 & 3 & 4 & 4 & 3 \\
\hline & Lituanie & 4 & 4 & 4 & 22 & 32 & 15 \\
\hline
\end{tabular}

(27) En dehors de quelques pays qui ont un registre de population de bonne qualité comme la Belgique, les Pays-Bas et les pays nordiques, et même dans ces cas, les sorties du territoire (radiations des registres) sont moins bien enregistrées que les entrées. 
Le nombre de sorties des natifs du pays dépasse souvent celui des départs de personnes ayant immigré quelques années auparavant dans le pays. Cependant, lorsque l'on prend en compte la taille de chaque population en calculant des taux d'émigration ${ }^{(28)}$, il apparaît nettement que les anciens immigrés sont plus mobiles que les natifs (tableau 14). En 2008, les taux d'émigration des natifs ne dépassent pas $4 \%$, sauf en Irlande (8\%o pour les hommes, $5 \%$ pour les femmes). La fréquence des départs d'immigrés varie selon les pays d'installation : limitée en Italie (9-10\%), elle dépasse $40 \%$ dans plusieurs pays (Irlande, Danemark, Autriche). Les hommes rentrent ou quittent leur pays plus souvent que les femmes, qu'il s'agisse de natifs ou d'immigrés. Seules les femmes immigrées en Espagne émigrent davantage. Mais les statistiques souffrent d'imperfections pour dégager des conclusions plus affinées.

La propension à repartir des immigrés est influencée par plusieurs facteurs. Les migrants originaires des pays développés sont plus nombreux à repartir que ceux venant des pays en développement. C'est en partie lié aux motifs qui les ont amenés dans le pays d'accueil, les premiers venant plus souvent pour des raisons économiques, les deuxièmes pour des raisons familiales ou humanitaires, comme l'illustre l'exemple de la Norvège (figure 38).

Figure 38. Proportion d'immigrants en Norvège repartis avant le $1^{\text {er }}$ janvier 2010 selon leur année d'arrivée et le motif de leur admission ${ }^{(29)}$

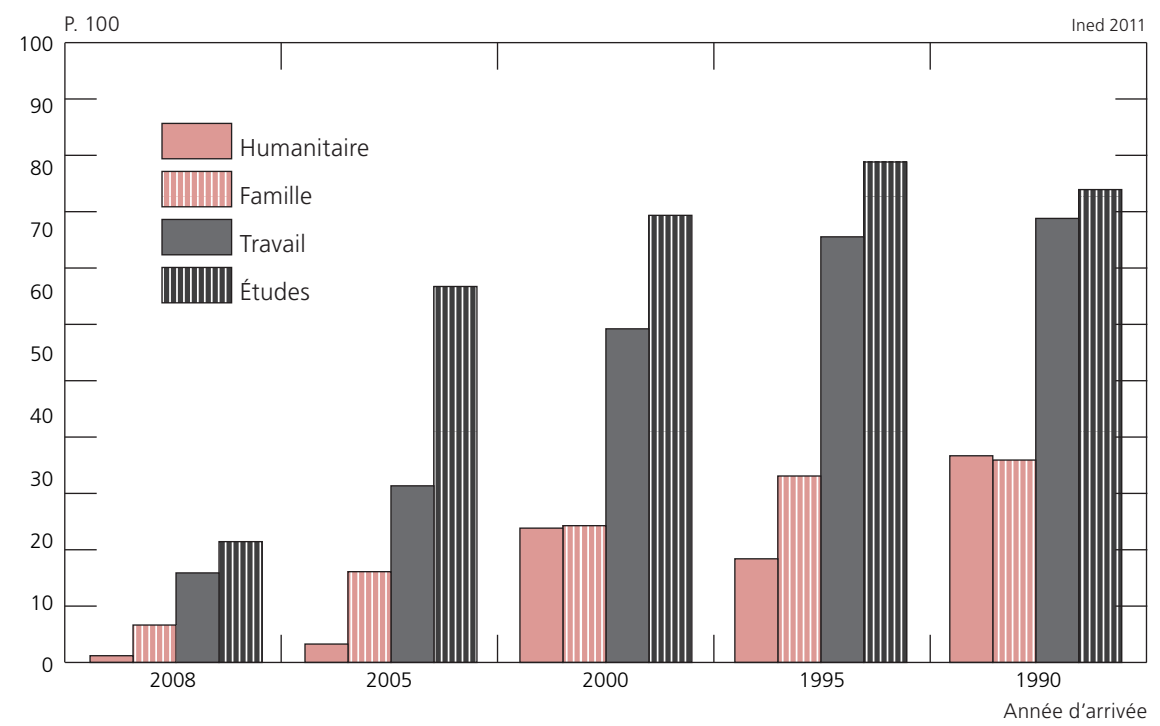

Lecture : Parmi les immigrants admis au séjour en 2008 pour des raisons humanitaires, seuls $1 \%$ sont repartis entre le moment de leur arrivée et le $1^{\text {er }}$ janvier 2010 . Cette proportion s'élève à $6 \%$ des immigrants familiaux, $15 \%$ des travailleurs et $20 \%$ des étudiants. Source : Statistics Norway.

(28) La répartition de la population totale par sexe et lieu de naissance n'étant disponible pour l'ensemble des pays qu'à partir de l'année 2009, il était impossible d'évaluer une population moyenne pour l'année 2008 pour le calcul des taux. Les taux d'émigration sont donc calculés comme le rapport des émigrations en 2008 à la population au $1^{\text {er }}$ janvier 2009, en faisant l'hypothèse que les effectifs ont peu changé entre le milieu de l'année 2008 et le début de l'année 2009.

(29) Il s'agit uniquement d'immigrants étrangers de nationalité non nordique. 
La proportion de départs observés dans une cohorte d'immigrants dépend de son ancienneté dans le pays. Parmi les immigrants arrivés en Norvège en 1995 ou avant, quatre immigrants sur dix ne résidaient plus dans le pays au $1^{\text {er }}$ janvier 2010 ; ce n'est le cas que d'un immigrant sur dix parmi ceux arrivés en 2008. Ces proportions sont également affectées par le motif d'admission. Les étudiants repartent plus vite que les travailleurs (60\% des premiers sont repartis au cours des cinq premières années, contre $30 \%$ des seconds), mais après 20 années de séjour les proportions s'égalisent (25\% vivent toujours en Norvège). Les migrants venus pour raisons familiales et humanitaires se distinguent par les proportions relativement faibles d'émigrés : depuis leur arrivée en Norvège en 1990, moins d'un tiers de ces immigrants seraient repartis du pays. Une situation similaire a été observée aux Pays-Bas : parmi les immigrants entrés en 1997, plus de $80 \%$ des étudiants et plus de $70 \%$ des travailleurs sont repartis au cours des six années suivantes, contre moins de $20 \%$ des migrants familiaux et des demandeurs d'asile (Nicolaas et Sprangers, 2004).

Outre les caractéristiques individuelles des migrants, celles des pays d'accueil ont également un impact sur la décision de repartir. Selon une étude de l'OCDE (2008), au sein de l'UE, 60 \% des immigrés en Irlande et $50 \%$ en Belgique seraient repartis au cours des cinq années suivant leur arrivée, contre seulement $28 \%$ de ceux venus aux Pays-Bas (tableau 15). En Amérique du Nord, les immigrés repartent moins souvent du pays d'accueil (19\% aux États-Unis et $24 \%$ au Canada, selon une étude à peine plus ancienne). Ces derniers, pays de forte immigration, sont aussi ceux dans lesquels l'installation définitive est plus fréquente qu'en Europe, qui se caractérise par une moindre attractivité.

Tableau 15. Proportion d'émigrants (\%) repartis durant les cinq premières années de résidence (population immigrée âgée de 15 ans ou plus)

\begin{tabular}{|l|c|c|}
\hline \multicolumn{1}{|c|}{ Pays } & Période d'entrée & Taux d'émigration après 5 ans (\%) \\
\hline Irlande & $1993-1998$ & 60,4 \\
Belgique & $1993-1999$ & 50,4 \\
Royaume-Uni & $1992-1998$ & 39,9 \\
Norvège & $1996-1999$ & 39,6 \\
Pays-Bas & $1994-1998$ & 28,2 \\
États-Unis & 1999 & 19,1 \\
\hline \multicolumn{2}{|l|}{ Source : OCDE (2008, p. 171, tableau III.1). } \\
\hline
\end{tabular}

\section{$X$. Les immigrés et leurs descendants}

Les entrées et sorties de migrants au cours d'une année contribuent à l'accroissement de la population durant cette période. Lorsqu'on souhaite analyser l'impact des migrations à plus long terme, d'autres indicateurs sont nécessaires. Le nombre d'immigrés et leur poids dans l'ensemble de la population résulte du cumul des mouvements migratoires sur plusieurs années, voire des 
décennies. La proportion de personnes issues de l'immigration (les immigrés, mais aussi leurs descendants nés dans le pays d'accueil) dans une population donnée est la combinaison de ces deux indicateurs, à laquelle s'ajoute le comportement démographique spécifique des immigrés, notamment en matière de fécondité.

\section{Les immigrés forment de $1 \%$ à $32 \%$ de la population totale selon les pays}

Les effectifs d'immigrés sont généralement mieux connus que les flux. Les principaux outils pour les identifier et les comptabiliser (recensements, registres de population) sont plus anciens, plus simples et moins coûteux que les outils mesurant les flux (registres de permis de séjour, enquêtes aux frontières). Les Nations unies publient les stocks de migrants pour l'ensemble des pays du monde (Nations unies, 2009a ; Pison, 2010). L'OCDE détaille ces statistiques selon l'âge, le niveau d'éducation et la durée de résidence des immigrés (Dumont et Spielvogel, 2008).

Deux critères sont utilisés pour identifier la population issue des migrations : le lieu de naissance pour distinguer la population immigrée et la nationalité pour la population étrangère ${ }^{(30)}$. De la même manière que pour l'étude des flux, l'utilisation de la variable lieu de naissance (qui reste identique durant la vie d'un individu) ou nationalité (qui peut changer dans le temps) ne conduit pas aux mêmes estimations de cette population, même si les deux ont un socle commun. Les étrangers sont majoritairement des immigrés ; cependant, dans les pays où la nationalité repose sur le droit du sang (Allemagne, Autriche, Suisse), il peut y avoir une population importante de natifs du pays de nationalité étrangère. La population immigrée se compose d'immigrés étrangers, mais aussi d'immigrés ayant acquis la nationalité du pays d'accueil (nationaux). Les statistiques selon le critère du lieu de naissance fournissent une meilleure vision des phénomènes touchant la population issue des migrations, mais ce sont les statistiques par nationalité qui restent, là aussi, les plus accessibles.

La population immigrée est constituée par des cohortes arrivées pendant plusieurs décennies. Les évolutions décrites dans la section précédente (accroissement du nombre de migrants, apparition de nouvelles destinations et origines...), s'observent aussi au niveau de la population immigrée, mais avec un décalage temporel dû à l'inertie démographique. L'exemple des migrations turques illustre bien ce phénomène. Le nombre d'arrivées d'immigrants originaires de Turquie est en diminution au niveau européen ; en Allemagne il a baissé de $50 \%$ entre

(30) Cependant la définition d'immigré utilisée dans les statistiques nationales contient parfois un critère de nationalité. Dans ce cas, les personnes nées à l'étranger avec la nationalité du pays en question (par exemple en France et aux États-Unis) ne seront pas considérées comme immigrées. En France, le Haut conseil à l'intégration définit un immigré comme « une personne née étrangère à l'étranger et résidant en France ». Les personnes nées françaises à l'étranger et vivant en France ne font donc pas partie de la population immigrée. 
1998 et 2008. Cette migration, la plus nombreuse auparavant, est désormais dépassée par celle d'autres origines. Néanmoins, les Turcs restent le groupe le plus important d'étrangers (7,9\%) dans l'UE (Vasileva, 2009).

L'amplification des mouvements migratoires, surtout à partir de la fin des années 1980, s'est traduite par un accroissement du stock d'immigrés en Europe : leur proportion dans l'ensemble de la population européenne passe d'environ 6 \% en 1990 à 10 \% en 2010 (Nations unies, 2009b). Parallèlement, les migrants se répartissent différemment au sein de l'Europe. En 1990, 70 \% des migrants résidaient en Europe de l'Ouest, proportion qui n'est plus que de 61 \% en 2010. Une proportion non négligeable de migrants se concentre désormais dans les pays d'Europe du Sud (28\%).

La proportion d'immigrés (d'individus nés à l'étranger) dans l'ensemble de la population est l'indicateur le plus souvent utilisé pour résumer ce bilan migratoire. En 2009, elle se situait entre $1 \%$ et $32 \%$ (tableau 16). Dans les pays d'Europe centrale, cette proportion n'atteint pas $5 \%$, conséquence d'une faible ouverture aux migrations dans le passé. Les pays traditionnels d'immigration (RoyaumeUni, France, Allemagne, Suède, Autriche) ont tous une proportion d'immigrés se situant entre $10 \%$ et $15 \%$. L'Espagne (14\% d'immigrés) affiche un niveau deux fois supérieur à celui de l'Italie et du Portugal, ce qui montre la rapidité des transformations induites par les migrations dans ce pays : en une décennie, il atteint le niveau des pays d'immigration ancienne. Les niveaux élevés en Lettonie et en Estonie résultent d'un artefact statistique : depuis la dissolution de l'URSS, les anciens migrants internes à cette région arrivés avant 1991 sont comptabilisés comme des migrants internationaux. La Suisse et le Luxembourg constituent des cas exceptionnels en Europe avec des proportions d'immigrés de $26 \%$ et $32 \%$, dont une majorité sont des ressortissants de l'UE.

Tableau 16. Proportion (\%) de personnes nées à l'étranger (immigrés) dans la population totale des pays en 2009

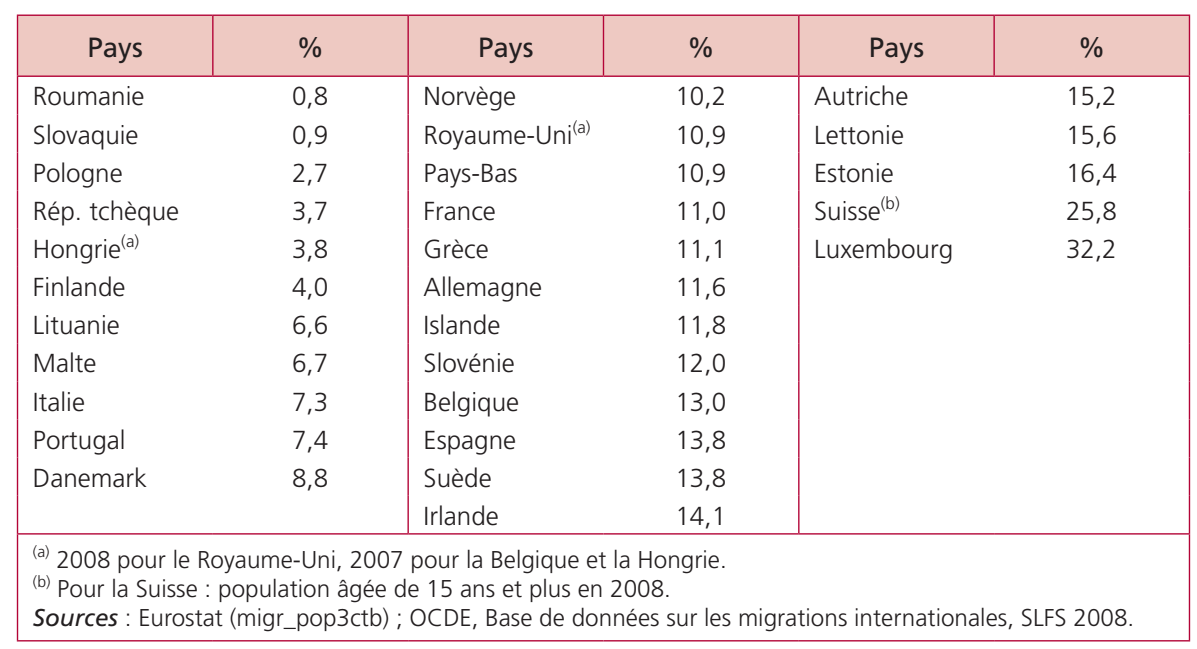


La diversification des origines nationales des migrants modifie peu à peu les caractéristiques de la population immigrée. L'exemple des cinq pays d'Europe du Nord illustre cette évolution. Jusque récemment, ces pays constituaient un système migratoire à part. Le Conseil nordique établi au lendemain de la deuxième guerre mondiale introduisit la libre circulation des personnes au sein de ces pays dès le début des années 1950, d'où la prépondérance des migrants des pays voisins par rapport aux migrants plus lointains. En Suède et en Norvège, principales destinations des populations nordiques, celles-ci constituaient respectivement $60 \%$ et $35 \%$ de la population étrangère au milieu des années 1970. Depuis, ces proportions se sont réduites dans l'ensemble de ces pays : comprises entre $15 \%$ et $40 \%$ en 1990, elles ne sont plus que $6 \%$ à $26 \%$ en 2008. Les origines géographiques plus diversifiées des nouveaux immigrants (tableau 11) expliquent une partie de cette baisse. Y contribue également la mobilité des immigrants nordiques, alors que ceux venant d'autres régions du monde sont plus sédentaires.

Certaines autres caractéristiques de la population immigrée (distribution par âge, proportion d'immigrés possédant la nationalité du pays d'accueil, etc.) sont directement liées à la durée de résidence des immigrés dans le pays d'accueil. En France et en Espagne, les immigrants récents sont plutôt jeunes (avec respectivement un âge moyen de 28 et 30 ans en 2008). Cependant, les populations immigrées dans les deux pays diffèrent considérablement. En France, la majorité des immigrés sont installés depuis plusieurs décennies ${ }^{(31)}$ et leur pyramide des âges est plus vieille (âge moyen de 47 ans) que celle des natifs (39 ans). Le nombre relativement faible de nouveaux immigrants ne permet pas de rajeunir cette sous-population, tandis que la population native se rajeunit en permanence par les naissances, qui sont relativement nombreuses en France ${ }^{(32)}$. La situation inverse s'observe en Espagne, où les arrivées nombreuses et continues de jeunes immigrants produisent une pyramide des âges d'immigrés plus jeune (âge moyen de 36 ans) que celle des natifs (42 ans).

\section{La fécondité des immigrés converge vers celle des natifs}

La contribution des migrants à la démographie de la population d'accueil ne s'arrête pas au moment de l'immigration. Leurs comportements en matière de fécondité, mise en union ou mobilité ont un impact plus ou moins important sur ces phénomènes observés dans la population totale. Ici les analyses portent plus particulièrement sur la fécondité des migrants et la formation des populations issues de l'immigration, mais nées dans le pays d'accueil ${ }^{(33)}$.

(31) Au moment du recensement de 1999, 83 \% des immigrés résidaient en France depuis 10 ans ou plus.

(32) Notons que le niveau relativement élevé de la fécondité en France (2,0 enfants par femme en 2010) n'est que marginalement dû à la présence des femmes étrangères, puisque leur apport est estimé à 0,1 enfant environ (Héran et Pison, 2007).

(33) Leurs appellations sont nombreuses : enfants d'immigrés, descendants d'immigrés, secondes générations... 
Les migrants arrivant ou circulant en Europe sont plutôt jeunes et donc en âge d'avoir des enfants. Qu'ils soient déjà parents dans le pays d'origine ou pas, beaucoup d'entre eux compléteront leur descendance une fois installés dans le pays d'accueil. La figure 39 montre l'évolution de la proportion de naissances issues des mères étrangères dans quelques pays européens depuis $1995^{(34)}$.

Figure 39. Proportion de naissances de mères étrangères dans l'ensemble de naissances depuis 1995 (\%)

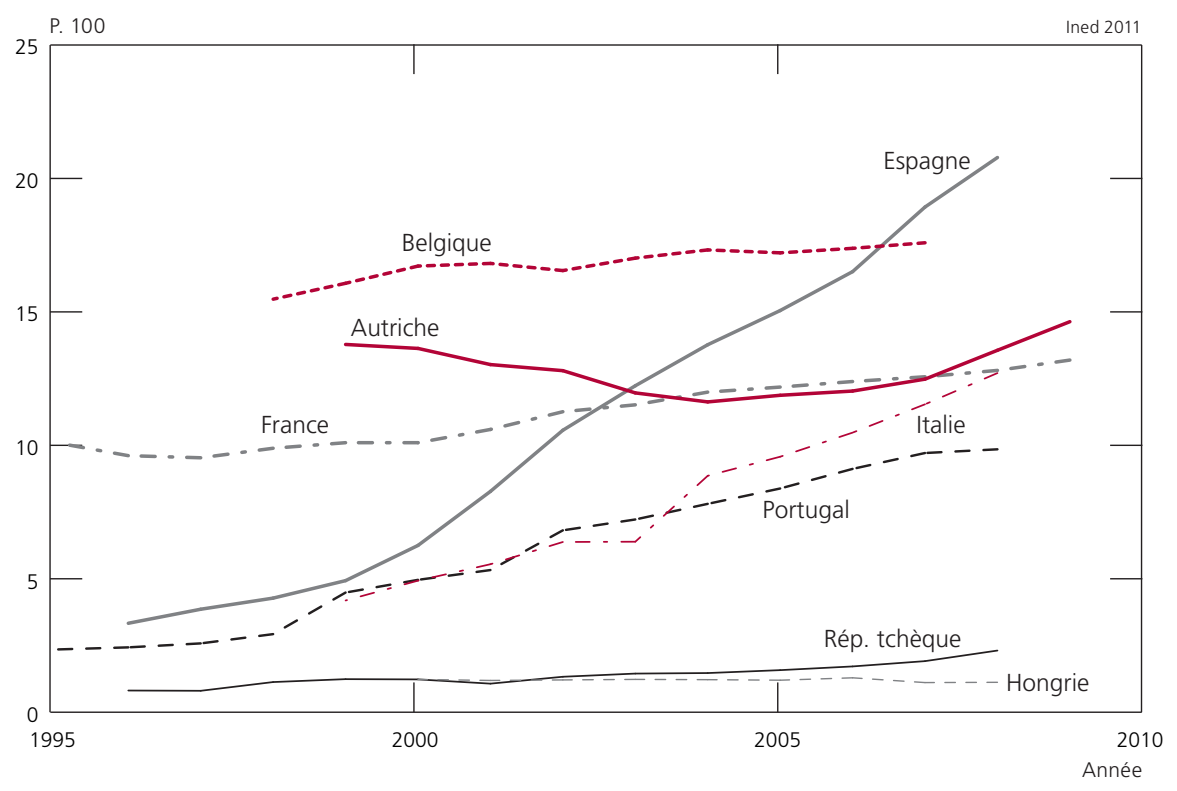

Sources: Instituts nationaux de statistique.

Les anciens pays d'immigration tels que la France, la Belgique et l'Autriche affichent des proportions relativement stables de naissances de mères étrangères. En France, cet indicateur oscille autour de 10 \% depuis les années 1980 ; il s'est accru depuis les années 2000 pour atteindre $13 \%$ en 2008. En Belgique, il est légèrement supérieur (15 \% à la fin des années 1990) et a également augmenté dans la période récente. La Suisse et le Luxembourg, deux pays ayant une proportion importante d'immigrés (tableau 16), ont également des niveaux exceptionnels de naissances d'étrangères (respectivement $36 \%$ et $56 \%$ en 2008). Les nouveaux pays d'immigration en Europe du Sud se caractérisent par la forte augmentation du poids des naissances de mères étrangères : jusqu'à la fin des années 1990 moins d'un enfant sur vingt naissait d'une mère étrangère ; en 2008, c'est une naissance sur cinq en Espagne, une naissance sur huit en Italie et une naissance sur dix au Portugal. Les naissances de mères étrangères

(34) Cet indicateur ne mesure pas la part des naissances issues de mères immigrées (dont certaines ont pu acquérir la nationalité de leur pays d'accueil) qui sont plus nombreuses. 
restent peu nombreuses dans les pays d'Europe centrale, de l'ordre de $1 \%$ à $2 \%$ de l'ensemble. Cependant, en République tchèque, cette proportion a triplé durant la période considérée.

Plusieurs facteurs sont à l'origine de l'accroissement de la contribution des immigrés (ou des étrangers) à la natalité, dont la différence du niveau de fécondité n'est qu'un des aspects. Étant donné leur plus jeune structure par âge, la proportion de femmes en âge de procréer est plus importante parmi la population immigrée. Par conséquent, son taux de natalité ${ }^{(35)}$ est plus élevé que celui de la population native, surtout dans le contexte de vieillissement des populations européennes. En Espagne, le taux de natalité des étrangers (21\%o) est à présent deux fois supérieur à celui des nationaux (10\%) (Vila et Martin, 2007).

L'indicateur conjoncturel de fécondité permet de comparer l'intensité de la fécondité des deux populations, en faisant l'hypothèse d'une répartition par âge identique. Le niveau de fécondité des femmes étrangères varie selon les pays d'accueil : au milieu des années 2000, il allait de 1,7 enfant par femme en Allemagne et en Espagne à 3,3 en France (Sobotka, 2010). Il est systématiquement plus élevé que celui des femmes ayant la nationalité du pays d'accueil, et les écarts avec ces dernières vont de 0,4 enfant par femme (Espagne) à 1,5 (France). Cependant, le calcul sur la population étrangère donne une vision biaisée de la fécondité des migrants étant donné qu'elle n'inclut pas la fécondité des migrants naturalisés, plus proche de la fécondité des natives. Les niveaux de fécondité des femmes immigrées, disponibles pour un nombre plus restreint de pays, sont moins élevés et leurs écarts avec les femmes natives sont moindres. Par exemple, en Suède en 2008, l'ICF était de 2,6 enfants par femme parmi les femmes étrangères, de 2,1 parmi les femmes immigrées et de 1,9 parmi les femmes natives (Sobotka, 2010).

Quatre facteurs sont évoqués dans la littérature pour expliquer ces écarts ${ }^{(36)}$. Les caractéristiques sélectives des migrants (leur niveau d'éducation, leur taux d'intermariage) favoriseraient une fécondité plus importante. La socialisation dans les pays d'origine, dont le niveau de fécondité est souvent supérieur à celui du pays d'accueil, serait un deuxième facteur. Le lien entre migration et formation de la famille expliquerait les niveaux particulièrement élevés de fécondité dans les premières années après l'arrivée dans le pays ${ }^{(37)}$ (Davie et Mazuy, 2010 ; Tribalat, 2005 ; Toulemon et Mazuy, 2004 ; Ostby, 2002). En dernier lieu, la persistance d'une haute fécondité serait une réaction de défense

(35) Le taux de natalité est le rapport du nombre de naissances de l'année à la population totale moyenne de l'année.

(36) Voir Sobotka (2010) pour une synthèse des études existantes au sujet de la natalité et de la fécondité des migrants.

(37) Les indicateurs de fécondité calculés de façon transversale tendent à surestimer la fécondité des migrants et accentuent donc leurs différences avec la population native. Une prise en compte de la durée de résidence dans le pays d'accueil s'avère nécessaire, surtout lors de l'estimation de la fécondité parmi les cohortes arrivées récemment. 
des minorités n’adhérant pas aux idéaux de famille de la population majoritaire (« l'état de minorité ») (38).

Les études adoptant une perspective longitudinale mettent en lumière les processus de convergence entre les niveaux de fécondité des immigrées et des natives. Le tableau 17 présente le nombre moyen d'enfants par femme aux PaysBas suivant leur ascendance migratoire. La fécondité de l'ensemble des femmes a augmenté de 1,5 à 1,8 enfant durant ces quinze dernières années. Parallèlement, celle des femmes immigrées a baissé de 2,1 enfants par femme en 1996 à 1,8 en 2009, après avoir atteint un maximum de 2,3 en 2000. Par conséquent les niveaux de fécondité des deux groupes se sont rapprochés : l'écart, de 0,6 au début de la période, est désormais proche de zéro.

Tableau 17. Indicateur conjoncturel de fécondité aux Pays-Bas depuis 1996 selon l'origine migratoire des femmes

\begin{tabular}{|l|c|c|c|c|}
\hline \multirow{2}{*}{ Année } & $\begin{array}{c}\text { Ensemble } \\
\text { des femmes }\end{array}$ & $\begin{array}{c}\text { Femmes dont les deux } \\
\text { parents sont nés aux Pays-Bas } \\
\text { (natives sans origine } \\
\text { migratoire) }\end{array}$ & $\begin{array}{c}\text { Femmes dont au moins un parent } \\
\text { est né à l'étranger } \\
\text { (immigrées et descendantes } \\
\text { d'immigrés) }\end{array}$ \\
\cline { 4 - 5 } & 1,5 & 1,5 & 1,8 & dont immigrées \\
\hline 1996 & 1,7 & 1,7 & 2,0 & 2,1 \\
2000 & 1,7 & 1,7 & 1,8 & 2,3 \\
2005 & 1,8 & 1,8 & 1,8 & 2,0 \\
2009 & & Ensemble & 1,8 \\
\hline
\end{tabular}

Source : SCBS Statline-Geboorte; herkomstgroepering en leeftijd moeder (op 31 december).

\section{La difficile estimation des populations issues de l'immigration}

Que la fécondité des migrants soit ou non supérieure à celle des natifs, le nombre de personnes nées de parents migrants et leur proportion dans la population totale augmentent inévitablement lorsqu'un pays voit croître sa population immigrée. Une fois la proportion d'immigrés stabilisée et en l'absence d'un écart de fécondité trop important entre les deux groupes, la proportion de descendants d'immigrants devrait elle aussi se stabiliser. C'est en partie ce que l'on observe dans la figure 39 : dans les pays traditionnels d'immigration d'Europe de l'Ouest, les proportions de naissances de mères étrangères évoluent peu dans la période analysée, alors qu'elles sont en rapide augmentation en Europe du Sud.

Aujourd'hui seuls quelques pays disposent de données sur la composition de leur population par origine, avec la distinction non seulement des immigrés, mais aussi de leurs descendants (Tribalat, 2008). Cela tient au caractère récent des flux dans beaucoup de pays européens ; les deuxièmes générations n'ayant pas encore eu le temps de se constituer, il n'y a pas de collecte statistique les

(38) Pour d'autres auteurs, cette même situation « d'état de minorité » expliquerait la limitation de naissances, permettant aux migrants d'accroître leur mobilité sociale et économique plus rapidement. 
concernant. Mais il existe aussi un problème plus général lié aux données. Le calcul d'un tel indicateur nécessite des informations systématiques non seulement sur la personne concernée, mais aussi sur ses parents. Les registres de population permettent une telle compilation de données. Les pays sans registre de population sont obligés de mener des enquêtes spécialisées pour arriver à une estimation ponctuelle du même type.

La figure 40 présente l'évolution de la proportion des immigrés et de leurs descendants $^{(39)}$ dans la population totale de deux pays, le Danemark et les Pays-Bas.

Figure 40. Proportion des populations issues de l'immigration dans la population totale du Danemark depuis 1980 (A) et des Pays-Bas depuis 1995 (B)

A. Danemark

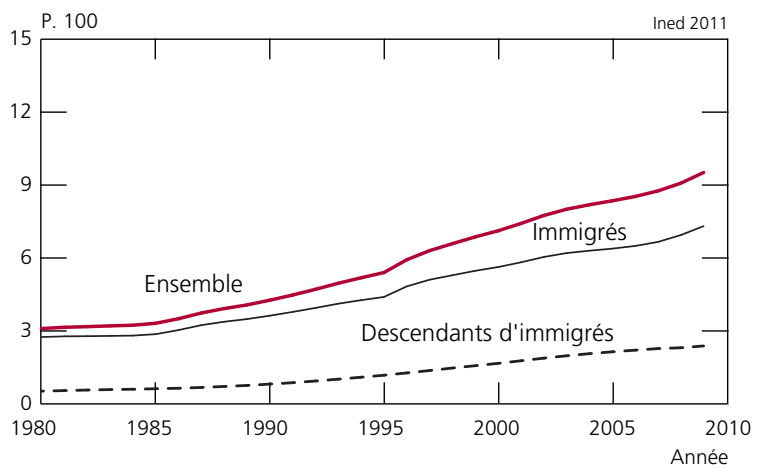

B. Pays-Bas

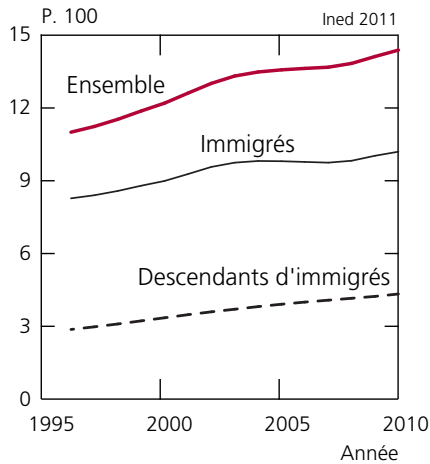

Sources: Statistics Denmark ; Statistics Netherlands.

Le Danemark a connu un développement de l'immigration plus tardif que la France, l'Allemagne ou les Pays-Bas. Depuis les années 1980, le nombre annuel d'entrées d'étrangers est en augmentation et se situe entre 30000 et 50000 depuis le milieu des années 1990. La population immigrée a donc beaucoup augmenté durant la période, de 135000 en 1980 à 350000 en 2006, soit en moyenne de 4,4 \% par an sur la période. Parallèlement, le nombre de descendants d'immigrés est passé de 18000 à 113000 à raison de $+7,3 \%$ en moyenne par an. Le nombre de personnes « sans ascendance migratoire » étant resté inchangé pendant la période (4,9 millions), la part de la population d'origine immigrée dans la population totale est passée de $3 \%$ à $9 \%$ en 25 ans. Aujourd'hui, la composition de la population par origine de ce pays ressemble à celle des Pays-Bas 15 ans auparavant.

L'immigration aux Pays-Bas a démarré dès la fin de la guerre. On peut faire l'hypothèse que sa composition par origine a suivi la même évolution qu'au

(39) Un descendant est défini comme une personne dont les deux parents sont soit immigrés, soit descendants avec une nationalité étrangère. 
Danemark, mais plus tôt. Depuis les années 1990, les populations immigrées et descendantes d'immigrés continuent de s'accroître, bien qu'à un rythme moins soutenu qu'au Danemark, alors que la population native stagne ${ }^{(40)}$. Les immigrés représentent à présent 10,3 \% de la population totale et leurs descendants 4,3\% si l'on ne prend en compte, comme au Danemark, que les descendants dont les deux parents sont immigrés. Si les enfants dont un seul parent est immigré sont inclus dans le nombre de descendants leur proportion atteint $10 \%$ Le tableau 18 présente la proportion de différents sous-groupes issus de l'immigration dans la population totale pour cinq pays européens.

Tableau 18. Proportion (\%) de la population issue de l'immigration dans la population totale en 2008

\begin{tabular}{|c|c|c|c|c|}
\hline \multirow[b]{2}{*}{ Pays } & \multirow[b]{2}{*}{ Immigrés } & \multicolumn{2}{|c|}{ Descendants d'immigrés dont } & \multirow[b]{2}{*}{ Ensemble } \\
\hline & & $\begin{array}{l}\text { les deux parents } \\
\text { sont immigrés }\end{array}$ & $\begin{array}{l}\text { un seul parent } \\
\text { est immigré }\end{array}$ & \\
\hline Pays-Bas & 10 & 4 & 6 & 20 \\
\hline France & 8 & 5 & 6 & 19 \\
\hline Autriche & 13 & 4 & non disponible & 17 \\
\hline Norvège ${ }^{(a)}$ & 9 & 2 & 4 & 16 \\
\hline Danemark & 7 & 2 & non disponible & 9 \\
\hline
\end{tabular}

Les résultats pour l'Autriche et le Danemark ne sont pas complètement comparables aux autres pays car ils n'estiment pas les populations issues d'un seul parent immigré (enfants de couples mixtes). Pour les trois autres pays, on observe que la proportion d'enfants de couples mixtes est toujours un peu supérieure à celle d'enfants dont les deux parents sont immigrés ; c'est une distinction importante à faire car les conditions de socialisation de ces deux groupes d'enfants sont différentes. Il apparaît aussi que le rapport entre la première et la deuxième génération de la population d'origine immigrée dépend directement de l'ancienneté des flux migratoires dans le pays : en France les descendants d'immigrés dépassent désormais les immigrés (11\% contre $8 \%$ ), alors que la situation est inverse en Norvège (6\% contre $9 \%$ ).

Des projections analysant l'impact des migrations à plus long terme sur les populations d'accueil et estimant la proportion des personnes d'origine immigrée $^{(41)}$ dans la population totale ont été menées dans quelques pays : Autriche, Royaume-Uni, Danemark, Allemagne, Pays-Bas, Norvège et Suède (Coleman, 2006). Au début des années 2000, cette proportion se situait entre

(40) Entre 1996 et 2010, un taux d'accroissement moyen de 2,0\% a été observé pour les immigrés, de 3,6 \% pour les descendants d'immigrés et $0,1 \%$ pour les autres.

(41) Pour une discussion des différentes définitions utilisées pour construire cette population voir Coleman (2006) et Tribalat (2008). 
$9 \%$ et $17 \%$ dans les pays analysés et elle atteindrait entre $15 \%$ et $32 \%$ en 2050. Cette évolution amène l'auteur à parler d'une troisième transition démographique dans les populations d'accueil caractérisées par une fécondité basse et une immigration élevée, se traduisant par une diversification de la composition des populations par origine.

\section{Conclusion : l'avenir de la population européenne}

L'avenir démographique de la population européenne est éclairé par les projections de population telles que peuvent les construire les pays ou des organismes internationaux comme les Nations unies et Eurostat. Le but ici est moins de faire un pronostic sur l'évolution la plus vraisemblable que de montrer quels seront les facteurs à l'œuvre dans les parcours à venir. De ce point de vue, la révision 2008 des projections des Nations unies offre un instrument commode, en proposant des jeux d'hypothèses cohérents dans les différents pays et des combinaisons d'hypothèses faisant apparaître les composantes à venir de la croissance démographique.

En matière de fécondité trois hypothèses sont envisagées. Dans l'hypothèse moyenne, il est supposé que tous les pays du monde convergent progressivement vers un indicateur annuel de 1,85 enfant par femme. En Europe, ce niveau serait atteint presque partout avant la fin de la projection en 2050. Dans l'hypothèse haute, le terme n'est plus 1,85 mais 2,35 enfants par femme. Dans l'hypothèse basse, c'est 1,35 .

La mortalité est supposée continuer à reculer progressivement. L'espérance de vie à la naissance (sexes réunis) passerait par exemple de 81 ans en 20102015 à 85 ans en 2045-2050 en Europe occidentale. Les gains sont un peu plus rapides dans les pays où l'espérance de vie est plus basse. D'où, ici aussi, une convergence. Une alternative à cette hypothèse est proposée, qui suppose une mortalité constante à son plus récent niveau, sans amélioration ni dégradation.

Enfin, les niveaux futurs de migration internationale sont établis « sur la base des estimations passées et en tenant compte des politiques engagées par chaque pays dans ce domaine. Les niveaux projetés sont généralement maintenus constants sur la majeure partie de la période de projection. » Lalternative est ici une migration nulle chaque année de 2010 à 2050, l'émigration contrebalançant l'immigration.

Le scénario de référence combine fécondité moyenne, mortalité en recul et migration non nulle. Deux autre scénarios ne diffèrent de celui-ci que par les hypothèses de fécondité, celle-ci étant haute dans un cas et basse dans l'autre. La comparaison des résultats obtenus dans ces trois scénarios permet d'apprécier le rôle de la fécondité dans les évolutions futures.

Deux scénarios sont ajoutés, qui ont en commun une fécondité moyenne. L'un diffère du scénario de référence parce qu'il n'y a pas de recul de mortalité, l'autre parce que la migration est nulle. La comparaison des résultats avec la 
référence permet d'apprécier respectivement l'impact du recul de la mortalité et celui de la migration.

\section{1. Évolution de la population totale}

En Europe (au sens des Nations unies, mais sans le Belarus, la Russie et l'Ukraine), la population totale s'accroîtrait lentement jusque vers 2025 (passant de 537 à 546 millions d'habitants) avant de décroître progressivement jusqu'au terme de la projection en 2050 (de 546 à 533 millions) ${ }^{(42)}$ (figure 41).

Figure 41. Différents scénarios de croissance de la population européenne jusqu'en 2050

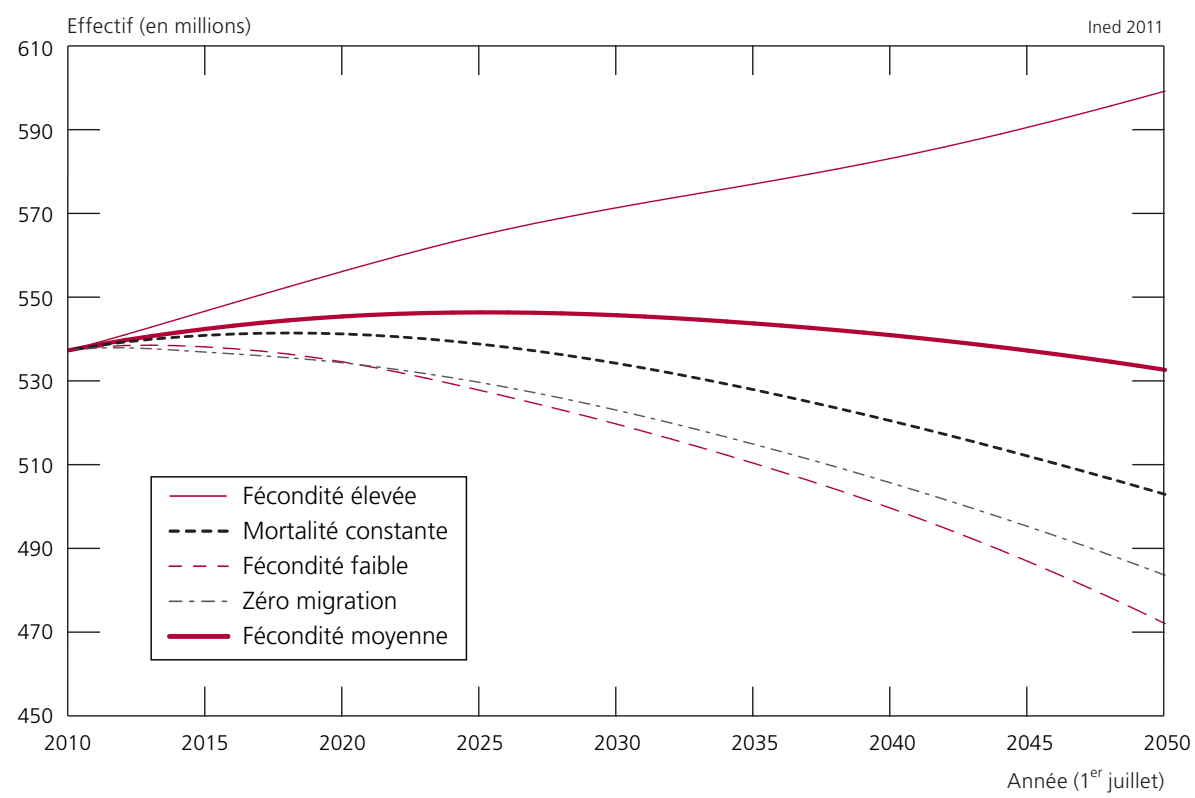

Source : Nations unies (2009c).

Une fécondité plus élevée ou moins élevée modifierait radicalement ce parcours. Avec une fécondité haute, l'effectif de population s'accroîtrait continuellement et rapidement, pour atteindre 600 millions en 2050. Avec une fécondité basse, il diminuerait presque immédiatement et sans discontinuer, pour n'être plus que 472 millions en 2050. La fourchette est large, mais elle reflète bien l'écart qui sépare des fécondités de 2,35 et 1,35 enfants par femme.

Si la mortalité ne baissait pas, la population s'accroîtrait encore quelques années avant de reculer jusqu'à 503 millions en 2050. C'est 30 millions de moins que dans le scénario de référence, dans lequel la fécondité est moyenne et la mortalité en recul. Les gains futurs contre la mortalité fourniraient donc

(42) Avec le Belarus, la Russie et l'Ukraine, la population de l'Europe passerait de 733 à 691 millions d'habitants entre 2010 et 2050. La population de Chypre passerait de 0,9 à 1,2 million d'habitants. 
30 millions d'habitants supplémentaires à la population européenne. C'est la moitié du « manque à gagner » que provoquerait une baisse de fécondité vers un niveau moyen de 1,35 enfant par femme.

Si la migration internationale était nulle, la population européenne décroîtrait presque immédiatement, à une allure très voisine de celle adoptée par le scénario de fécondité basse. L'effectif en 2050 serait de 483 millions d'habitants. Ce serait alors 50 millions de moins que dans le scénario de référence. Lapport migratoire équivaut à peu près au « manque à gagner » que provoquerait une baisse de fécondité vers un niveau moyen de 1,35 enfant par femme.

Malgré la convergence des hypothèses de fécondité et de mortalité vers des niveaux communs, le parcours des pays européens promet d'être sensiblement différent dans les décennies à venir, du fait de conditions initiales contrastées. La population d'Europe du Nord devrait s'accroître de 10 \% d'ici à 2050, alors que celle d'Europe centrale et orientale devrait largement décroître (respectivement de $15 \%$ et $20 \%)^{(43)}$. Ces deux mouvements de sens opposé devraient être continus sur la période. Au contraire, les deux autres régions, Sud et Ouest, devraient voir leur population augmenter légèrement puis diminuer lentement, comme le continent dans son ensemble (figure 42).

Figure 42. Projections des effectifs de population des régions européennes selon l'hypothèse de fécondité moyenne, 2010-2050 (indice 1 en 2010)

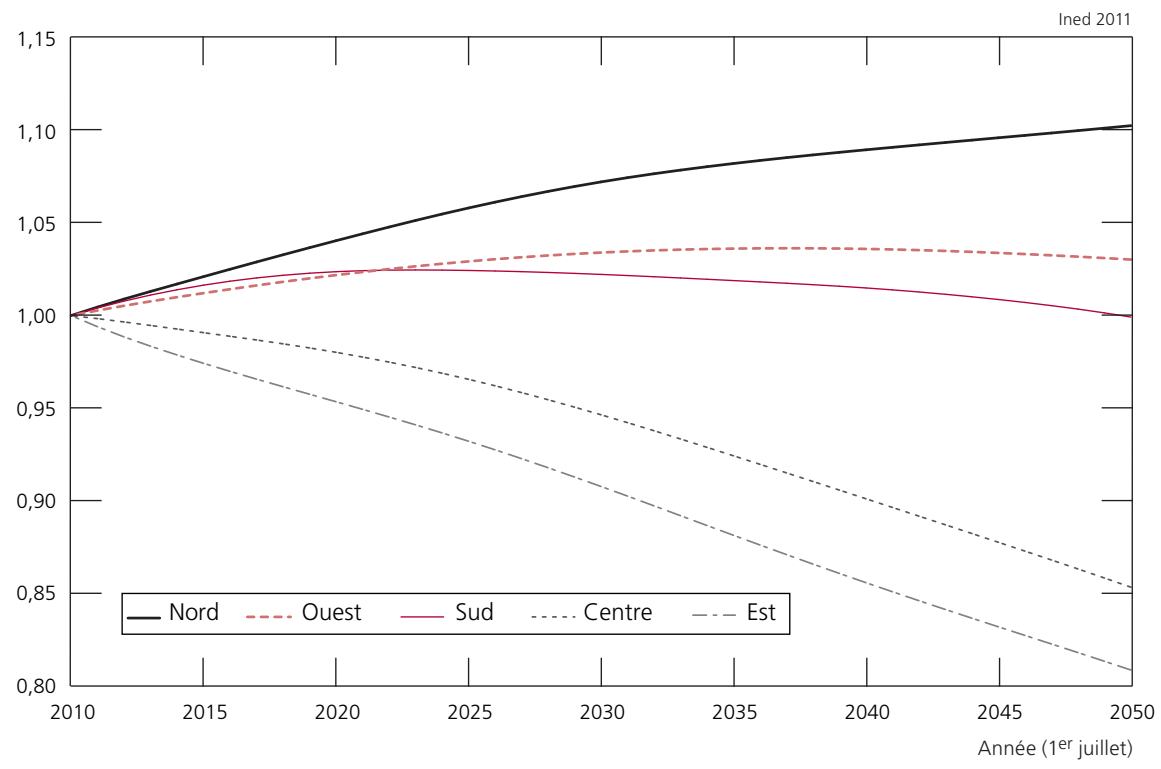

Source : Nations unies (2009c).

(43) L'évolution au Belarus, en Russie et en Ukraine ne diffère pas de celle des autres pays d'Europe de l'Est. 
L'opposition entre le Nord d'une part et le Centre et l'Est d'autre part se prolonge dans une analyse des composantes de la croissance dans chaque région. $\mathrm{Au}$ Nord, même avec une fécondité basse, la population en 2050 serait plus nombreuse qu'en 2010, la baisse de la mortalité jouant un rôle modeste (car l'espérance de vie est élevée dès aujourd'hui) et l'apport migratoire un rôle important. Au Centre et à l'Est, même avec une fécondité haute, la population serait en recul ; les gains d'espérance de vie ont un impact plus visible qu'au Nord, mais l'apport migratoire est négligeable. Le Sud et l'Ouest ont des profils d'évolution proches, essentiellement différenciés par une contribution plus large des migrations à la dynamique démographique dans les pays méridionaux.

\section{2. Évolution de la population âgée}

L'augmentation de la part des personnes âgées affectera presque inéluctablement l'Europe dans les décennies à venir. En 2010, 17 \% de la population européenne est âgée de 65 ans ou plus et ce pourcentage dépasserait $25 \%$ en 2050 dans tous les scénarios; il pourrait même dépasser $32 \%$ dans certaines configurations d'hypothèses. Dans le scénario de référence, ce serait plus de

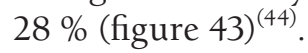

Figure 43. Proportion (\%) d'Européens âgés de 65 ans ou plus, selon différentes hypothèses de projection, 2010-2050

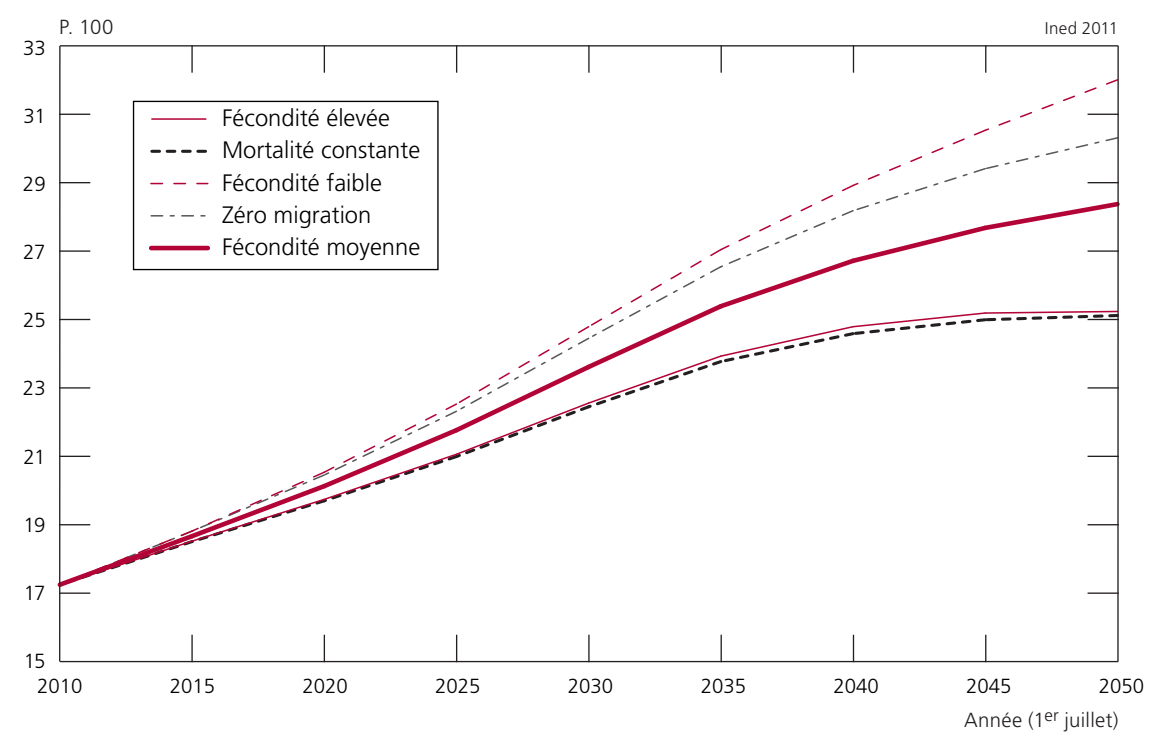

Source : Nations unies (2009c).

Le niveau de fécondité joue, on le sait, un rôle important dans le degré de vieillissement des populations. On le vérifie ici puisque la proportion de

(44) Dans une définition de l'Europe qui englobe aussi le Belarus, la Russie et l'Ukraine, les différents pourcentages diminuent tous d'un point. 
personnes âgées passe de $25 \%$ à $32 \%$ en 2050 selon que la fécondité est haute ou basse. On notera qu'avec une fécondité partout plus élevée qu'aujourd'hui (hypothèse haute), le degré de vieillissement s'accentue malgré tout sensiblement. Ce qu'on pourrait exprimer un peu différemment en disant : avec une fécondité plus élevée qu'aujourd'hui, le vieillissement serait inférieur de trois points à ce qu'il serait dans le scénario de référence, soit une progression de huit points au lieu de onze. C'est une modulation à la marge.

L'ampleur de la modulation est la même sous l'effet de la mortalité. Si celle-ci ne baissait pas, la proportion de personnes âgées atteindrait $25 \%$. Le recul de la mortalité fait passer ce chiffre à $28 \%$, car il provoque un surcroît de vieillissement. Le rôle de la migration est inverse : si la population européenne ne recevait pas d'apport extérieur, la proportion de personnes âgées dépasserait $30 \%$, soit deux points de plus que dans le scénario de référence. L'existence d'une immigration nette en Europe freinerait le vieillissement de la population. Une fois de plus, ce serait à la marge. Le vieillissement apparaît inéluctable, car même la combinaison des trois freins identifiés ici ne saurait l'empêcher de se produire.

La dynamique du vieillissement diffère cependant entre les régions européennes. Pour le Nord et l'Ouest du continent, le scénario de référence fait apparaître un ralentissement du vieillissement des populations à partir de 2040 ; pour le Sud, il n'y a d'inflexion qu'une dizaine d'années plus tard ; pour le Centre et l'Est ${ }^{(45)}$, ce serait plutôt une accélération en fin de période de projection (figure 44).

Figure 44. Proportion (\%) d'Européens âgés de 65 ans ou plus dans les différentes régions selon I'hypothèse de fécondité moyenne, 2010-2050

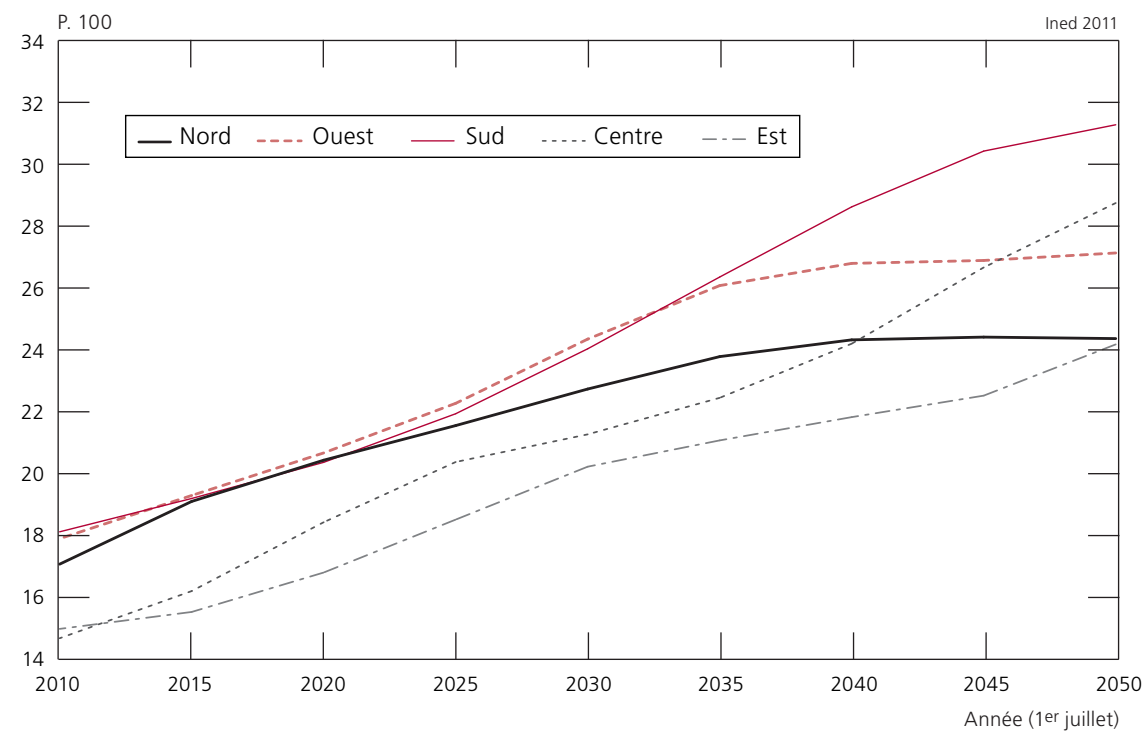

Source : Nations unies (2009c).

(45) L'évolution au Belarus, en Russie et en Ukraine ne diffère pas de celle des autres pays d'Europe de l'Est. 
Ces évolutions nous renvoient à la chronique de la fécondité 65 ans auparavant, quand se sont formées les générations qui vont concourir à la population âgée à venir. À partir de 1975, au Nord et à l'Ouest, c'était la période du « babykrach », époque à laquelle le nombre de naissances était au plus bas. Ces générations peu nombreuses n'ajouteront que des effectifs réduits à la population âgée à partir de 2040. En Europe du Sud, ce même processus se reproduira avec un décalage d'une dizaine d'années, car la baisse de la fécondité a été plus tardive. Dans le Centre et l'Est, les années 1970-1980 étaient au contraire des périodes de fécondité relativement soutenue, sous l'effet de politiques natalistes parfois généreuses, d'autres fois répressives; d'où une augmentation rapide du nombre de personnes âgées à prévoir dans les années 2040.

Ceci nous rappelle que les évolutions démographiques sont lentes à produire leurs effets. Même si on postule - davantage qu'on ne vérifie - une convergence des comportements, il faudra du temps avant que les populations des différentes régions d'Europe se transforment au même rythme. 


\section{ANNEXE : AUX SOURCES DE LA DÉMOGRAPHIE DE L'EUROPE}

Ce chapitre présente une vue générale des principales sources statistiques mobilisables pour analyser la situation démographique européenne, complété par un panorama des méthodes de recensement des populations qui ont sensiblement évolué dans les dernières décennies, et par un tour d'horizon des données d'enquête, à disposition de ceux qui souhaitent affiner le portrait des populations européennes dans une perspective comparative.

Quasiment tous les pays européens disposent d'une statistique fiable du mouvement de population et de longues séries de données démographiques. La plupart des indicateurs utilisés dans cette chronique proviennent de la collection de données lancée par Alain Monnier et poursuivie par Alexandre Avdeev. Elle a été récemment transformée en une base accessible au public sur le site de l'Ined ${ }^{(46)}$, et sur celui du Centre d'études démographiques de l'Université d'État de Moscou ${ }^{(47)}$. Une partie de cette base contient des séries allant du début de $\mathrm{XX}^{\mathrm{e}}$ siècle à nos jours. Ces données sont puisées en majeure partie dans les publications officielles des agences statistiques nationales et dans la collection des données de l'Observatoire démographique européen fournies par Gérard Calot et Jean-Paul Sardon et complétées, dans certains cas, par les données d'Eurostat ${ }^{(48)}$.

Les statistiques nationales et les indicateurs du mouvement de population des pays européens sont comparables, au moins pour la période postérieure à la deuxième guerre mondiale. Cependant, l'effectif de la population, hormis lorsqu'il est issu des recensements généraux, est toujours une estimation et comporte un certain degré d'incertitude. Cela concerne particulièrement les pays des Balkans et les pays avec une migration internationale importante ${ }^{(49)}$.

Les plus petits pays (Monaco, Saint-Marin, Vatican) ont été exclus de l'analyse. Quant à l'analyse des composantes de l'évolution de la population, elle n'a pas été effectuée pour le Liechtenstein et Andorre, car les fluctuations des nombres d'événements démographiques y ont souvent une amplitude trop importante par rapport à l'effectif de leur population.

(46) http://www.ined.fr/fr/pop_chiffres/pays_developpes/base_pays_developpes/

(47) http://devision-dmo.econ.msu.ru/

(48) http://epp.eurostat.ec.europa.eu/portal/page/portal/statistics/search_database

(49) Ainsi, les recensements de l'Albanie (2001) et du Belarus (2009) ont révélé une population bien inférieure aux estimations précensitaires, sans que celles-ci soient ultérieurement révisées. De telles situations sont exclues de notre analyse. Par ailleurs, les données de la province autonome du Kosovo et de Metohija ayant été exclues de la statistique serbe en 1998, elles le sont de notre analyse de la croissance de la population et de ses composants pour les deux dernières décennies. Les estimations officielles de la population du Kosovo sont néanmoins prises en compte dans l'analyse de la dynamique de l'ensemble de la population de l'Europe. 
La présentation cartographique s'appuie sur une analyse de la répartition des pays selon le niveau de différents indicateurs :

- $10 \%(5 \%+5 \%)$ de pays aux deux extrémités de la répartition ;

- 50 \% de pays au milieu de la distribution, formant les deuxième et troisième quartiles;

- $40 \%(20 \%+20 \%)$ des pays se trouvent à droite et à gauche du groupe central. Nous distinguons aussi les pays qui se trouvent à droite et gauche d'un indicateur de tendance centrale (la médiane ou la moyenne arithmétique).

Les moyennes utilisées ici sont de deux types. Les moyennes du premier type sont obtenues en divisant la somme des indicateurs nationaux par le nombre de pays ; c'est la moyenne « politique », qui caractérise la situation démographique des États européens indépendamment de l'effectif de leur population. Dans les moyennes arithmétiques du second type, les indicateurs nationaux sont pondérés par l'effectif de la population de chaque pays ; c'est la moyenne « démographique » caractérisant l'état de la population européenne dans son ensemble, indépendamment des frontières nationales.

Les bases de données citées précédemment sont complétées par des bases spécifiques, touchant des domaines particuliers de la dynamique des populations. La plus large concerne la mortalité. La Human Mortality Database ${ }^{(50)}$, contient des résultats sous forme de taux et de tables de mortalité pour des populations nationales, ainsi que les éléments bruts ayant permis d'obtenir ces résultats. Des données relatives à 26 pays d'Europe sont ainsi disponibles. Une méthodologie unique a été utilisée dans tous les pays. Un complément est fourni par la Human Life Table Database ${ }^{(51)}$, dans laquelle les tables de mortalité ont été construites par des techniques diverses. Des résultats sont ainsi disponibles pour l'Albanie, Chypre, le Groenland et Malte.

Une base est en cours de construction pour la fécondité, la Human Fertility Database $^{(52)}$, mais au stade actuel elle ne couvre que huit pays d'Europe.

Pour la migration internationale, la constitution d'une base comparative est beaucoup plus délicate car l'hétérogénéité des sources est forte lorsqu'il s'agit de saisir les flux d'entrée et de sortie d'un pays, aussi bien pour les étrangers que pour les nationaux. Il s'y ajoute des divergences dans le concept même de migrant. Un point sur la situation a été effectué récemment dans le cadre du projet Prominstat (Promoting Comparative Quantitative Research in the Field of Migration and Integration in Europe; D. Kupiszewska et al., 2010). La difficulté de comparer les statistiques de migration en Europe amène à donner une vue d'ensemble en s'appuyant sur la mesure ou l'estimation des soldes migratoires, et à ne donner de détails sur les flux migratoires proprement dits (immigrations

\footnotetext{
(50) http://www.mortality.org/

(51) http://www.lifetable.de/

(52) http://www.humanfertility.org/
} 
et émigrations) que sur un nombre restreint d'exemples pour lesquels la qualité des données permet de les saisir correctement.

\section{Les recensements en Europe}

L'Europe est l'un des berceaux des recensements modernes de population, dont les premiers datent du XVIII ${ }^{\mathrm{e}}$ siècle $^{(53)}$. Au cours du XX $\mathrm{X}^{\mathrm{e}}$ siècle, la forme la plus commune de recensement est dite classique ${ }^{(54)}$. Mais l'homogénéité des années 1950-1960, où tous les pays recensaient leur population en interrogeant l'ensemble des individus au cours d'une seule enquête à une date fixe, est révolue. L'Europe entre dans une nouvelle ère où les recensements de la population évoluent vers d'autres formes. Conséquence des coûts croissants et des réactions de la société civile ${ }^{(55)}$, la collecte directe et exhaustive des données est remplacée par d'autres méthodes de collectes (enquêtes, registres, sources administratives) et les techniques de saisie et de contrôle se modernisent. En l'espace de trente ans les recensements européens montrent une grande diversification de situations.

\section{De nouvelles techniques de recensements}

Les recensements classiques de la population, méthode retenue dans la quasi-totalité des pays européens jusque dans les années 1970, sont remplacés soit par des formules mixtes, associant enquêtes et/ou registres administratifs, soit par des formules alternatives recourant exclusivement à des registres de population ou des nouvelles formules de recensement (France : rotation sur 5 ans des communes de petite taille et sondage dans les autres) ${ }^{(56)}$ (tableau 19).

\section{L'utilisation des registres}

Le degré et la forme d'utilisation des registres par les bureaux de statistiques nationaux sont très variés. Pour certains pays, ils sont la base même du recensement, pour d'autres ils ne sont qu'un complément.

La Norvège adopta dès 1970 une méthode mixte de collecte, basée conjointement sur le registre de population mis en place en 1964, sur les registres de l'éducation et des salaires et sur un recensement de type classique.

(53) Un dénombrement fut conduit en Islande, alors sous administration danoise, dès 1703. La Suède (comprenant aussi la Finlande) organisa un recensement général de la population en 1749 , suivie par le Danemark en 1769 puis la France et l'Angleterre en 1801.

(54) Les recensements classiques consistent en un dénombrement complet de la population et des logements sur le terrain à partir d'un formulaire long. Traditionnellement, chaque individu était recensé en fonction de ses caractéristiques propres et les données étaient collectées sans avoir recours à des techniques de sondage.

(55) Le cas de l'Allemagne est exemplaire. En effet, suite à des mouvements de contestations au nom de la défense des libertés individuelles, les dirigeants allemands ont reporté le recensement prévu au début de la décennie 1980 ; puis le rapprochement des deux Allemagnes augmentant considérablement la population à recenser et donc les coûts du recensement, aucune opération exhaustive n'a été menée de 1987 (RFA) à 2011.

(56) Pour une présentation des différentes méthodes de recensement, voir Valente (2010). 
Tableau 19. Évolution des formes de recensement en Europe

\begin{tabular}{|c|c|c|c|}
\hline \multirow{2}{*}{ Pays } & \multicolumn{3}{|c|}{ Vagues de recensement } \\
\hline & 1985-1994 & $1995-2004$ & 2005-2014 \\
\hline Albanie & 12/04/1989 & $01 / 04 / 2001$ & $01 / 10 / 2011$ \\
\hline \multirow[t]{2}{*}{ Allemagne } & 25/05/1987 (RFA) & 30/09/1995 (ménages) & \multirow[t]{2}{*}{ 09/05/2011 } \\
\hline & & $05 / 12 / 2001^{(\mathrm{a})}$ & \\
\hline Autriche & $15 / 05 / 1991$ & $15 / 05 / 2001$ & $31 / 10 / 2011$ \\
\hline Belgique & $01 / 03 / 1991$ & $01 / 10 / 2001$ & $01 / 01 / 2011$ \\
\hline Bosnie-Herzégovine & $31 / 03 / 1991$ & & $01-15 / 04 / 2011$ \\
\hline Bulgarie & $\begin{array}{l}04 / 12 / 1985 \\
04 / 12 / 1992\end{array}$ & $01 / 03 / 2001$ & $01-28 / 02 / 2011$ \\
\hline Chypre & 01/10/1992 & $01 / 10 / 2001$ & 2011 \\
\hline Croatie & $31 / 03 / 1991$ & $31 / 03 / 2001$ & $01-15 / 04 / 2011$ \\
\hline Danemark & $01 / 01 / 1991$ & $01 / 01 / 2001$ & $01 / 01 / 2011$ \\
\hline Espagne & 01/03/1991 & $01 / 11 / 2001$ & $01 / 11 / 2011$ \\
\hline Estonie & $12 / 01 / 1989$ & $31 / 03 / 2000$ & $31 / 12 / 2011-31 / 03 / 2012$ \\
\hline \multirow[t]{2}{*}{ Finlande } & $17 / 11 / 1985$ & $31 / 12 / 1995$ & \multirow[t]{2}{*}{$31 / 12 / 2010$} \\
\hline & $31 / 12 / 1990$ & $31 / 12 / 2000$ & \\
\hline France & 05/03/1990 & 08/03/1999 & $\begin{array}{c}20 / 01 / 2006 \\
\text { et années suivantes }\end{array}$ \\
\hline Grèce & 17/03/1991 & $18 / 03 / 2001$ & $16-18 / 03 / 2011$ \\
\hline Hongrie & 01/01/1990 & $01 / 02 / 2001$ & $01 / 10-30 / 11 / 2011$ \\
\hline \multirow[t]{2}{*}{ Irlande } & 13/04/1986 & 28/04/1996 & $23 / 04 / 2006$ \\
\hline & 21/04/1991 & $28 / 04 / 2002$ & $10 / 04 / 2011$ \\
\hline Islande & (b) & (b) & 2011 \\
\hline Italie & 20/10/1991 & $21 / 10 / 2001$ & 09/10/2011 \\
\hline Lettonie & $12 / 01 / 1989$ & 06/04/2001 & $01 / 03 / 2011$ \\
\hline Lituanie & 12/01/1989 & 06/04/2001 & $01 / 03 / 2011$ \\
\hline Luxembourg & 01/03/1991 & $15 / 02 / 2001$ & $01 / 02 / 2011$ \\
\hline Macédoine & $31 / 03 / 1991$ & $01 / 11 / 2002$ & $10-11 / 2011^{(c)}$ \\
\hline Malte & $16 / 11 / 1985$ & 26/11/1995 & $\begin{array}{c}27 / 11 / 2005 \\
21 / 11-18 / 12 / 2011 \\
\end{array}$ \\
\hline Moldavie & 12/01/1989 & 05/10/2004 & $01 / 04 / 2012$ \\
\hline Monténégro & $31 / 03 / 1991$ & $31 / 10 / 2003$ & $01-15 / 04 / 2011$ \\
\hline Norvège & 03/11/1990 & $03 / 11 / 2001$ & $19 / 11 / 2011$ \\
\hline Pays-Bas & 01/01/1991 & $01 / 01 / 2001$ & $01 / 01 / 2011$ \\
\hline Pologne & $06 / 12 / 1988$ & $20 / 05 / 2002$ & $01 / 04-30 / 06 / 2011$ \\
\hline Portugal & 15/04/1991 & $12 / 03 / 2001$ & $21 / 03 / 2011$ \\
\hline République tchèque & 03/03/1991 & $01 / 03 / 2001$ & $25-26 / 03 / 2011$ \\
\hline Roumanie & 07/01/1992 & $18 / 03 / 2002$ & $10 / 2011$ \\
\hline Royaume-Uni & 21/04/1991 & 29/04/2001 & 27/03/2011 \\
\hline Serbie & $31 / 03 / 1991$ & $31 / 03 / 2002$ & $01 / 04 / 2011$ \\
\hline Slovaquie & 03/03/1991 & 25/05/2001 & $21 / 05 / 2011$ \\
\hline Slovénie & $31 / 03 / 1991$ & $31 / 03 / 2002$ & $01 / 01 / 2011$ \\
\hline Suède & $\begin{array}{l}01 / 11 / 1985 \\
01 / 11 / 1990 \\
\end{array}$ & (b) & $31 / 12 / 2011$ \\
\hline Suisse & 04/12/1990 & $05 / 12 / 2000$ & $31 / 12 / 2010$ \\
\hline \multicolumn{4}{|c|}{$\begin{array}{l}\text { Note : Les dates des recensements non effectués (recensement prévus courant } 2011 \text { ou 2012) peuvent être } \\
\text { modifiées, par conséquent les dates reportées ici sont celles annoncées officiellement au } 15 \text { mars } 2011 \text {. Ce sont } \\
\text { le plus souvent les dates de référence du recensement ou à défaut les dates d'enquêtes. } \\
\text { (a) Organisation du test d'un recensement basé sur les registres le } 5 \text { décembre } 2001 \text { sur } 1,2 \% \text { de la } \\
\text { population. } \\
\text { (b) L'Islande n'a pas organisé de recensement de } 1981 \text { à 2011. La Suède n'a pas effectué de recensement durant } \\
\text { la vague } 1995-2004 \text {. } \\
\text { (c) Le } 09 \text { mars le recensement prévu le } 31 \text { mars } 2011 \text { a été reporté à l'automne } 2011 \text {. }\end{array}$} \\
\hline
\end{tabular}


En 1990, le recensement était issu de trois sources : les registres, une enquête par sondage sur l'emploi et les logements dans les villes de plus de 6000 habitants et un « recensement » des personnes de plus de 16 ans dans les villes plus petites (Eggerickx et Bégeot, 1993). En 2011, le recensement est finalement uniquement réalisé à partir des données des trois registres principaux : le registre central de population (incorporant désormais le registre de l'emploi et du chômage, celui de l'éducation, le registre issu de l'administration fiscale), le registre des entreprises et celui des adresses et habitations.

$\mathrm{Au}$ cours des quarante dernières années, les pays nordiques ont largement développé et généralisé l'utilisation des données administratives : le Danemark a opté pour un régime mixte de recensement en $1976^{(57)}$, la Suède et la Finlande en 1985. Ils sont devenus au cours du temps une référence pour de nombreux États pourvus de registres, comme l'office statistique de la République de Slovénie ${ }^{(58)}$. Les Pays- Bas dès 1971 et la Belgique en 1981 ont eux aussi adopté un système mixte de recensement.

Motivés par les économies que permet cette méthode, d'autres pays aspirent à développer la qualité de leur registre pour, dans un futur plus ou moins proche, pouvoir mener ce type de recensement. La Pologne prépare pour 2011 un recensement combinant des données des registres et un recensement par autodéclaration. Les données issues des registres proviendront de 28 sources, originaires des registres centraux mais aussi des registres municipaux et des administrations publiques. Selon une estimation faite par le bureau central des statistiques polonais, cette nouvelle méthode, associée à l'utilisation d'Internet, permettra de diminuer le nombre des agents recenseurs (de 200000 à 20000 en 2011) et les coûts de l'opération censitaire.

\section{Le recours aux sondages}

La méthode des sondages est utilisée soit sur l'ensemble de la population (l'Allemagne réunie mena en 1991 un recensement sur 1 \% de la population), soit sur une partie seulement de l'échantillon (le recensement français dans toutes les villes de plus de 10000 habitants), soit uniquement pour la collecte de certaines données socioéconomiques (utilisation de deux questionnaires, un court et un long administrés à une fraction de la population, cas de la Hongrie en 1990).

En 2011, l'Italie va utiliser deux types de formulaires dans les villes de 20000 habitants et plus : un court pour les caractéristiques démographiques et un long pour les variables socioéconomiques recueillies par sondage. Cette solution semble n'être qu'une étape dans la refonte du système de collecte de données démographiques et socioéconomiques, l'Italie souhaitant mettre en place dès 2021 un recensement uniquement fondé sur les registres.

(57) Puis, en 1981, le Danemark a mis en œuvre un recensement basé uniquement sur les registres.

(58) Conférence des statisticiens européens, « Moyens d'améliorer le dénombrement dans le cas d'un recensement effectué à partir des registres : Exemple de la Slovénie » Genève, 28-30 octobre 2009, p. 2. 


\section{De nouvelles méthodes de collecte}

Internet est probablement la révolution technique du cycle de recensements 2005-2014. Plusieurs pays, comme la Suisse ou l'Espagne, avaient déjà encouragé leurs habitants à utiliser cette méthode lors de la vague de recensement précédente. Lors du $16^{\mathrm{e}}$ recensement fédéral en Suisse, 4,2 \% de la population concernée avait choisi cette option ${ }^{(59)}$. L'office fédéral suisse a réitéré cette expérience lors de son recensement de décembre 2010. La Pologne, la Lettonie, l'Autriche et le Portugal encouragent aussi l'autodéclaration via Internet. Au total, 13 pays y ont eu recours.

\section{Diversité des techniques et des méthodes mais plus grande harmonisation des données}

L'ONU et Eurostat ${ }^{(60)}$ émettent pour chaque cycle de recensement des recommandations relatives à la forme et à la structure des recensements, aux informations souhaitables et aux données à collecter, ainsi qu'une liste de tableaux à fournir. Ces recommandations constituent un cadre dans lequel chaque pays établit ensuite ses propres règles.

Si la politique nationale a un impact décisif sur la conduite ou la lecture des résultats des recensements ${ }^{(61)}$, les recommandations internationales se veulent incitatives et nombre de pays les suivent à la lettre, à commencer par l'année de réalisation des recensements. Conformément au souhait des Nations unies repris par Eurostat, une trentaine de pays mèneront leur recensement en 2011.

De même, dans un souci d'harmonisation et de comparabilité des données nationales collectées, ces organisations internationales encouragent les pays à poser certaines questions clefs (caractéristiques essentielles définies dans le cahier des charges) ou orientent le choix des questions pour que les données collectées renvoient à des réalités similaires. Ces recommandations sont peutêtre celles qui rencontrent le plus de résistance ou de problèmes car les recensements nationaux répondent prioritairement aux obligations nationales. Néanmoins, l'harmonisation et la comparabilité des données n'ont cessé de s'accroître.

\section{L'accès aux résultats des recensements}

Des tableaux présentant les résultats des recensements peuvent être consultés sur la base de données d'Eurostat. Ils portent aussi bien sur la campagne

(59) Tous les ménages ne pouvaient s'autodéclarer par Internet. Tout dépendait de la commune de résidence et de la technique de recensement adoptée par la commune. Le pourcentage de 4,2\% a été calculé sur la seule population concernée.

(60) Le Journal officiel de l'Union européenne a adopté un texte intitulé Regultation (EC) No 763/2008 of the European Parliament and of the Council of 9 July 2008 on Population and Housing Census. Ce document de neuf articles établit les recommandations relatives à la collaboration statistique entre Eurostat et les États membres pour la vague de recensement 2005-2014.

(61) Ces interférences sont particulièrement sensibles lorsqu'il s'agit de dénombrer des groupes ethniques : les cas du Nigéria et du Liban sont des exemples types dans ce domaine, mais le recensement américain de 1980 fit aussi ressortir ces tensions entre pouvoir et objectifs scientifiques (Choldin, 1986). En Europe, on peut citer le cas de l'Irlande (Courbage, 2003). 
de recensements la plus récente (2000-2001) que sur la précédente (1990-1991). Ils sont organisés autour de cinq thèmes : structure de la population, population active, niveau d'instruction, ménages et logements. Une trentaine de pays étaient couverts en 2000-2001, une vingtaine dix ans plus tôt.

Par ailleurs, un accès est désormais possible non pas aux tableaux chiffrés tirés de l'exploitation des données de recensement mais à des données individuelles, ce qui permet à l'utilisateur de bénéficier d'une souplesse maximale pour conduire son analyse sans la contrainte d'une tabulation préprogrammée. Une base comparative pour les pays européens est organisée par le projet IECM (Integrated European Census Microdata) ${ }^{(62)}$. Elle ouvre la possibilité de traiter des échantillons de données individuelles (avec des regroupements en ménage) issus de recensements nationaux depuis les années 1960 pour une douzaine de pays d'Europe.

\section{Les enquêtes européennes}

\section{Évolution des données comparatives en Europe}

Les enquêtes sont une source de données importante pour les études des évolutions démographiques et des changements de comportements sous-jacents. Pendant les dernières décennies, l'intérêt grandissant pour des comparaisons entre pays a mené au développement de réseaux qui facilitent l'accès aux données pour permettre les études comparatives sur la base d'enquêtes nationales. Mais ces études restent compliquées à cause des différences dans les concepts, les définitions et les questionnaires. Il s'y ajoute, d'un pays et d'une enquête à l'autre, des variations dans la méthode d'échantillonnage, de collecte et dans le choix de la population de base. Ce sont autant d'obstacles pour les comparaisons transnationales.

Afin de remédier à ces problèmes, des enquêtes d'envergure européenne ont vu le jour pendant les dernières décennies, avec comme objectif principal la production de données comparatives sur des sujets divers. Ces enquêtes sont de mieux en mieux documentées et accessibles. Parfois, elles prennent la forme d'un panel ce qui permet d'obtenir des données longitudinales et d'étudier des causalités. Les enquêtes qui fournissent des données transversales sont souvent répétées à intervalle régulier pour rendre possible l'étude des tendances. De plus, ces enquêtes permettent souvent de tester de nouvelles approches pour résoudre les problèmes méthodologiques de collecte et d'analyse propres aux études comparatives ${ }^{(63)}$.

Cette section présente les infrastructures créées pour faciliter l'accès et l'exploitation des données européennes, un bref aperçu des différentes enquêtes, et enfin quelques pistes d'amélioration. Ces informations ont été recueillies

(62) http://www.iecm-project.org/

(63) Voir par exemple le projet COMPARE lié à l'enquête européenne SHARE. 
majoritairement sur des sites web durant l'été 2010 et ne sont évidemment pas exhaustives.

\section{Infrastructure pour l'archivage et accessibilité des données}

En France, l'accès aux données internationales et à leur documentation est facilité par les membres du réseau Quételet ${ }^{(64)}$ formé par les Archives de données issues de la statistique publique du Centre Maurice Halbwachs ${ }^{(65)}$ (CMH-ADISP), le Centre de données socio-politiques ${ }^{(66)}$ (CDSP) et le service des enquêtes de l' Institut national d'études démographiques ${ }^{(67)}$ (Ined). Ces institutions participent elles-mêmes à certaines enquêtes européennes ou internationales telles que l'Enquête sociale européenne ${ }^{(68)}$ (ESS) pour le CDSP, l'International Social Survey Program $^{(69)}$ (ISSP) pour le CMH-ADISP, et l'enquête Generation and Gender ${ }^{(70)}$ (GGS) pour l'Ined.

Le réseau Quételet participe, à travers le CDSP, à un portail européen d'archivage de données, le Council of European Social Science Data Archives ${ }^{(71)}$ (CESSDA). Ce réseau est impliqué dans la construction des grandes infrastructures européennes pour la recherche dans le but de consolider l'accès des chercheurs à l'ensemble des données en Europe. Allant plus loin, le Center for Comparative European Survey Data ${ }^{(72)}$ (CCESD) ne cherche pas seulement à faciliter l'accès aux données, mais s'intéresse aussi aux solutions techniques de la mise à disposition et aux nouvelles méthodes d'analyse dans un contexte « international » ou « inter-culturel».

Faciliter l'accès des chercheurs aux données des différentes enquêtes est aussi l'objectif principal de deux grands réseaux internationaux, l'International Federation of Data Organisation ${ }^{\text {(73) }}$ (IFDO), en partenariat avec le CESSDA, et l'Inter-University Consortium for Political and Social Research ${ }^{(74)}$ (ICPSR), dont le CDSP est le représentant français. Le ICPSR est l'archive la plus ancienne et la plus importante qui permet aux chercheurs d'accéder gratuitement aux enquêtes archivées. Il fournit également de l'assistance aux chercheurs pour l'identification des données pertinentes et pour la conduite de leurs projets de recherche.

D’autres initiatives concernant l'accessibilité et la comparabilité des données d'enquêtes existent au niveau européen. Par exemple, EQUALSOC ${ }^{(75)}$, un réseau

(64) http://www.reseau-quetelet.cnrs.fr/

(65) http://www.cmh.greco.ens.fr/adisp

(66) http://cdsp.sciences-po.fr/

(67) http://www.ined.fr/

(68) http://ess.sciencespo.com/

(69) http://www.issp-france.info/

(70) http://www-erfi.ined.fr/

(71) http://www.cessda.org/

(72) http://www.ccesd.ac.uk

(73) http://www.ifdo.org

(74) http://www.icpsr.umich.edu/icpsrweb/ICPSR/

(75) http://www.equalsoc.org/2 
d'excellence de la recherche qui porte sur le changement économique, la qualité de vie et la cohésion sociale, a inclus dans ses objectifs de faciliter l'accès aux données pour ses membres, de traiter des problèmes d'harmonisation et de comparabilité, et d'initier de jeunes chercheurs à la recherche comparative. Ces aspects sont sous la responsabilité du CMH qui héberge le Data Support Committee de ce projet. Un autre exemple des efforts d'harmonisation des données est constitué par deux projets du Centre d'études de populations, de pauvreté et de politiques socioéconomiques / International Network for Studies in Technology, Environment, Alternatives, Development (CEPS/INSTEAD) au Luxembourg : le Panel Comparability Project Database (PACO) qui avait comme objectif d'harmoniser des données de panel d'un certain nombre de pays pour la période 1983-1993 et le projet Community Household Panels for European Research $^{(76)}$ (CHER).

Une autre avancée importante est le développement de bases de données qui permettent d'identifier des enquêtes (nationales et internationales) dans lesquelles une question particulière a été utilisée. Une telle initiative est prise par le CDSP en collaboration avec le Centre de recherches politiques de Sciences $\mathrm{Po}^{(77)}$ (Cevipof) et le Centre d'études européennes ${ }^{(78)}$ (CEE). Le Economic and Social Data Service $^{(79)}$ (ESDS) mène des activités comparables au Royaume-Uni.

\section{Méthodes et concepts des enquêtes internationales}

En plus des difficultés dans l'accessibilité des données, les différences dans les concepts et méthodes limitent les comparaisons, d'où l'intérêt des enquêtes européennes ou internationales qui portent une attention particulière à la comparabilité des méthodes de sondage, des concepts, des définitions et à l'influence socioculturelle propre à chaque pays. Outre l'harmonisation de la collecte et des instruments, des méthodes adaptées pour des analyses comparatives sont nécessaires. Plusieurs initiatives sont mises en œuvre de façon à faire avancer la recherche dans ce domaine, et le nombre d'ouvrages et articles traitant ces sujets est croissant (Harkness et al., 2002 ; Hantrais, 2009 ; Vigour, 2005 ; Hoffmeyer-Zlotnik et Wolf, 2003).

Le Leibnitz Institute for the Social Sciences in Germany ${ }^{(80)}$ (GESIS) est très engagé dans ce type de recherche méthodologique. Par exemple, le premier volume de leur série de publications Survey Methodology traite du concept de ménage privé et son utilisation dans des enquêtes nationales et internationales (Hoffmeyer-Zlotnik et al., 2008). Par ailleurs, un groupe de chercheurs, actifs dans le domaine des enquêtes comparatives, s'est organisé sous forme d'atelier (Workshop on Comparative Survey Design and Implementation ${ }^{(81)}$, CSDI) afin de

(76) http://www.ceps.lu/documents/historique/ceps-instead.pdf

(77) http://www.cevipof.com/

(78) http://www.cee.sciences-po.fr/

(79) http://www.esds.ac.uk/international/

(80) http://www.gesis.org/

(81) http://www.csdiworkshop.org/ 
faire avancer la recherche sur les méthodes de sondage et d'analyse. Un autre exemple est le projet COMPARE ${ }^{(82)}$, attaché à l'enquête SHARE, qui teste l'utilité des vignettes dans le contexte d'une étude comparative, par exemple sur la satisfaction avec la vie ou l'appréciation subjective de la santé. Il s'agit des descriptions de situations hypothétiques, dont l'évaluation par les enquêtés est utilisée pour estimer le biais introduit, par exemple, par des normes culturelles différentes ${ }^{(83)}$. Dans d'autres cas, la recherche sur les méthodes comparatives fait partie intégrante du projet d'enquête, comme c'est le cas pour l'Enquête sociale européenne ${ }^{(84)}$. Lié à ce projet, un portail, le Macro Data Guide ${ }^{(85)}$, donne accès à des données macro, rendues comparables autant que possible, potentiellement utiles pour des analyses comparatives. Une base de données contextuelle est également créée autour de l'enquête GGS $^{(86)}$ dans le cadre du projet GGP.

\section{Les enquêtes comparatives européennes}

Le tableau 20 présente brièvement les enquêtes européennes les plus importantes selon leur sujet principal, le type de l'enquête (transversale, longitudinale, répétée), les pays inclus et la méthode d'harmonisation utilisée.

Ces dernières décennies, un nombre important d'enquêtes européennes ou internationales ont vu le jour, dont certaines sous forme d'un panel. Ceci est le cas de l'enquête Generation and Gender qui prévoit trois vagues espacées de trois ans, mais aussi de deux enquêtes d'Eurostat : le panel des ménages de la Communauté européenne (PCM) et l'EU-SILC. Une dernière enquête de ce type, la Survey of Health, Ageing and Retirement in Europe (SHARE), cible la population âgée de 50 ans et plus pour étudier les conditions de vie avant et après le passage en retraite.

D’autres enquêtes sont répétées régulièrement, ce qui permet, entre autres, d'étudier l'évolution dans le temps de certains comportements et opinions. L'enquête répétitive européenne la plus ancienne est l'Enquête sur les forces de travail (EFT), qui existe en France depuis les années 1950. La fréquence de cette enquête varie selon les pays : dans certains cas, les données sont produites par trimestre, dans d'autres sur une base annuelle. La European Social Survey (ESS) et la European Values Survey (EVS) font également partie de ce type d'enquêtes. Elles visent principalement l'étude des opinions et des attitudes concernant des sujets très variés, tout comme l'enquête Population Policy Acceptance Study (PPAS), mais avec une moindre couverture géographique et thématique. L'Eurobaromètre, dont la fréquence et le contenu dépendent des

(82) http://www.compare-project.org/

(83) http://gking.harvard.edu/vign/

(84) http://www.europeansocialsurvey.org/index.php?option=com_content\&view=article\&id=67 \&Itemid $=235$

(85) http://www.nsd.uib.no/macrodataguide/about.html

(86) http://www.ggp-i.org/contextual-database.html 
demandes formulées par la Commission européenne, change de sujet à chaque passage, même si des répétitions pour certains sujets particuliers permettent de suivre des tendances. Une collaboration internationale, la plus ancienne de ce type, est à la base de l'International Social Survey Programme ${ }^{(87)}$ (ISSP) qui vise la comparaison dans l'espace et dans le temps.

Un intérêt grandissant de la politique pour mesurer l'efficacité des différents systèmes éducatifs a mené à plusieurs évaluations des connaissances des élèves, mais aussi des adultes. L'Enquête sur l'éducation des adultes (EEA) d'Eurostat ${ }^{(88)}$ est un projet pilote, et l'OCDE prépare actuellement un Programme pour l'évaluation internationale des compétences des adultes (PIAAC). Ce programme inclut une enquête qui évaluera le niveau et la répartition des compétences des adultes d'une façon systématique et cohérente dans les différents pays. Après l'International Adult Literacy Survey (IALS) dans les années 1990 et l'Adult Literacy and Life Skills Survey (ALL), le PIAAC est la troisième enquête de l'OCDE sur cette thématique. Afin d'évaluer la réussite des enfants dans le système scolaire de chaque pays participant, plusieurs enquêtes coexistent. Citons en trois. D'abord une autre enquête de l'OCDE, le Programme for International Student Assessment (PISA) ${ }^{(89)}$ qui en est à son $4^{\mathrm{e}}$ cycle et qui teste l'acquisition de certaines capacités, considérées comme fondamentales, par des enfants âgés de 15 ans. Parallèlement sont recueillies des informations sur l'environnement familial et scolaire. C'est aussi le cas pour deux autres enquêtes ${ }^{(90)}$ - TIMSS et PIRLS - qui testent séparément les compétences en lecture, en compréhension et en sciences pour des groupes d'âges particuliers.

\section{La santé, la migration et la famille sont moins couverts par les enquêtes européennes}

Des enquêtes européennes existent sur des sujets très variés, tels que les conditions de travail (EWCS) ou la qualité de vie (EQLS) organisées par l'Eurofound(91) dont les données sont accessibles par Internet. La santé, la migration et la famille, par contre, font rarement l'objet d'une enquête particulière, bien que des questions les concernant soient souvent incluses dans des études générales ou traitant de thématiques différentes.

Pour améliorer la comparabilité des données sur la santé entre pays et dans le temps, une initiative d'Eurostat et de la Commission européenne (DG Health and Consumers) tente non seulement de mettre en place un système de collecte de données harmonisées, mais aussi de développer des modules de questions qui peuvent être utilisés dans des enquêtes européennes. Les détails sur le European Module on Health Status (EMHS), le European Survey Module on

\footnotetext{
(87) http://www.issp.org

(88) http://epp.eurostat.ec.europa.eu/portal/page/portal/microdata/adult_education_survey

(89) http://www.pisa.oecd.org

(90) http://timss.bc.edu/

(91) http://www.eurofound.europa.eu/surveys/index.htm
} 


\begin{tabular}{|c|c|c|c|c|c|c|c|c|c|c|c|}
\hline 芯 & $\overline{\bar{z}}$ & 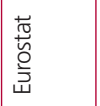 & $\begin{array}{l}\text { 苛 } \\
\text { ồ } \\
\text { 岦 }\end{array}$ & 岀 & 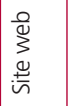 & 岕 & 岕 & 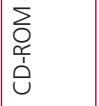 & 岕 & 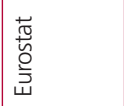 & 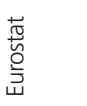 \\
\hline 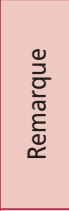 & 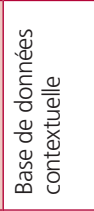 & 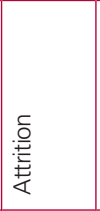 & 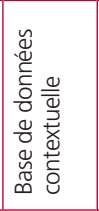 & 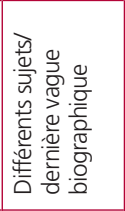 & 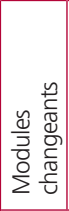 & & 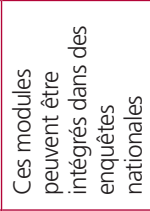 & & 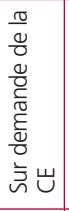 & & \\
\hline 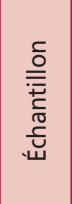 & 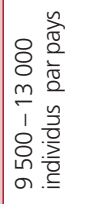 & 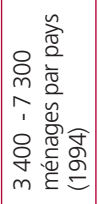 & 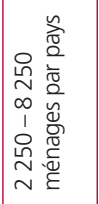 & 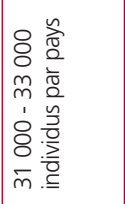 & 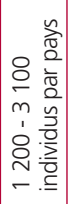 & 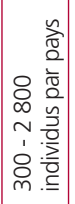 & 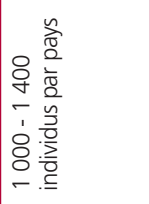 & 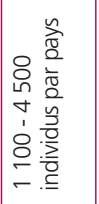 & 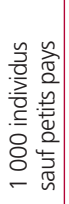 & 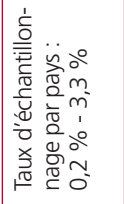 & 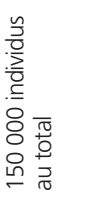 \\
\hline 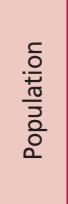 & $\begin{array}{l}\tilde{c} \\
\tilde{\sigma} \\
\widehat{\alpha} \\
\infty \\
-\end{array}$ & 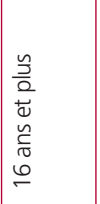 & 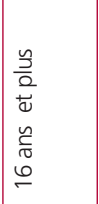 & $\begin{array}{l}\frac{n}{3} \\
\frac{a}{0} \\
d \\
\tilde{u} \\
\frac{n}{0} \\
0 \\
\text { in }\end{array}$ & 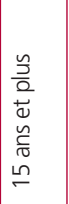 & 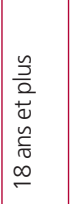 & 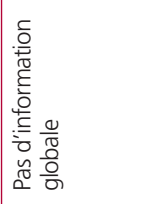 & 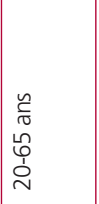 & 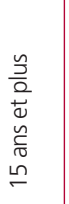 & 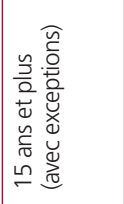 & 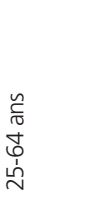 \\
\hline$\stackrel{ \pm}{\leftrightarrows}$ & 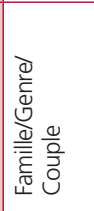 & 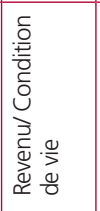 & 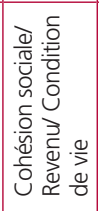 & 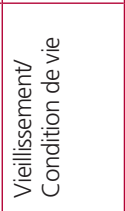 & 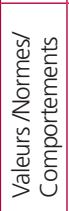 & $\frac{\tilde{y}}{\frac{\tilde{d}}{\tilde{N}}}$ & 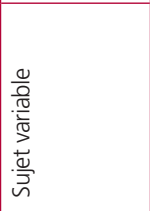 & 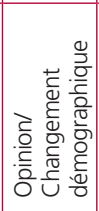 & 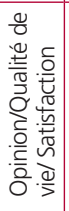 & 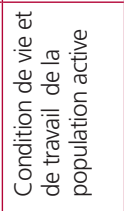 & 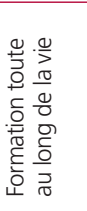 \\
\hline 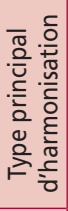 & 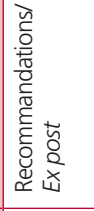 & 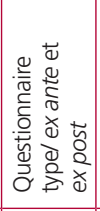 & 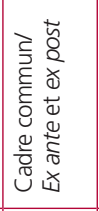 & 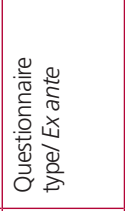 & 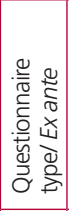 & 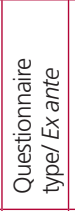 & 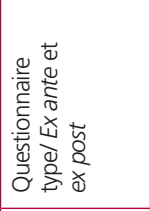 & 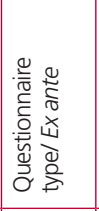 & 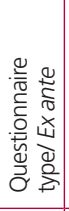 & 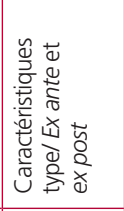 & 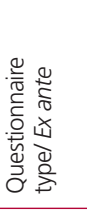 \\
\hline$\stackrel{\text { }}{\stackrel{n}{\sigma}}$ & 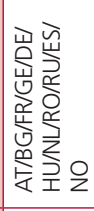 & 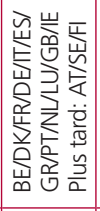 & 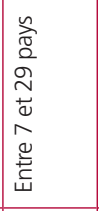 & 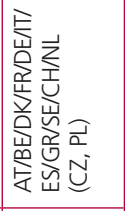 & 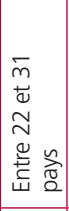 & 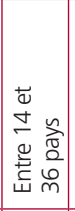 & 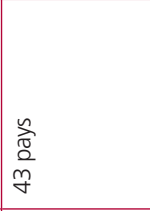 & 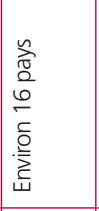 & 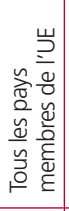 & 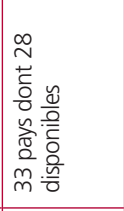 & 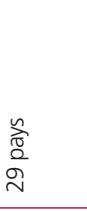 \\
\hline 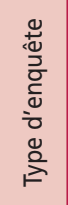 & 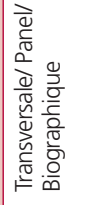 & 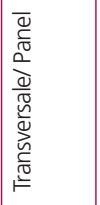 & 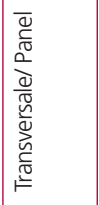 & 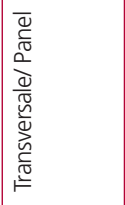 & 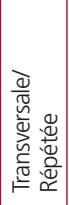 & 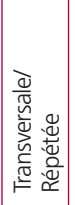 & 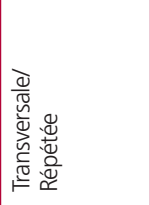 & 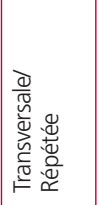 & 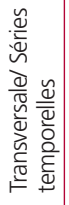 & 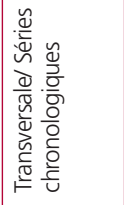 & $\begin{array}{l}\frac{0}{0} \\
\frac{0}{0} \\
\frac{0}{0} \\
\frac{0}{2}\end{array}$ \\
\hline ¿্ّ & 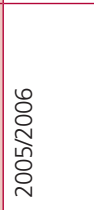 & 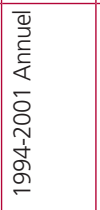 & 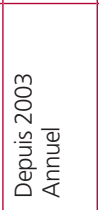 & 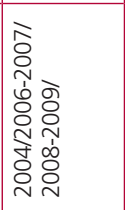 & 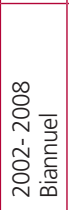 & 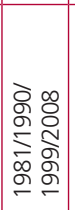 & 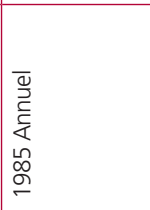 & 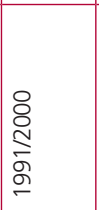 & 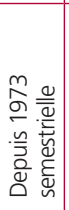 & 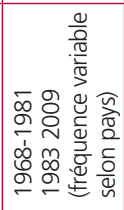 & 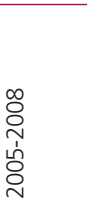 \\
\hline $\begin{array}{l}\varepsilon \\
\text { है }\end{array}$ & 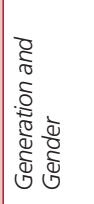 & 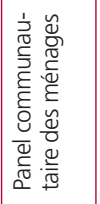 & 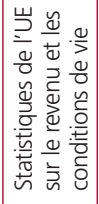 & 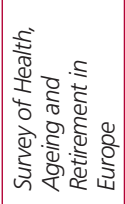 & 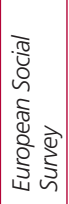 & 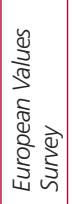 & 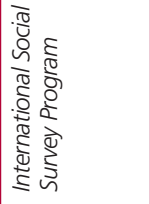 & 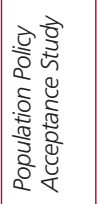 & 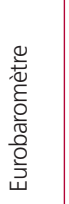 & 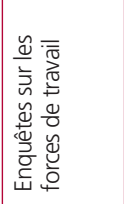 & 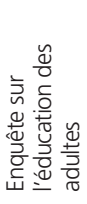 \\
\hline$\frac{0}{\pi}$ & 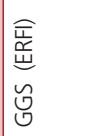 & 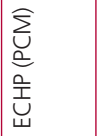 & $\begin{array}{l}\text { U. } \\
\text { ज् } \\
\text { w }\end{array}$ & $\begin{array}{l}\text { 愛 } \\
\text { 至 }\end{array}$ & 岀 & 岂 & $\underline{\approx}$ & 高 & & $\begin{array}{l}\text { 岳 } \\
\breve{u} \\
\text { 足 }\end{array}$ & 岃 \\
\hline
\end{tabular}




\begin{tabular}{|c|c|c|c|c|c|}
\hline 芯 & 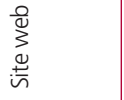 & 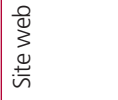 & 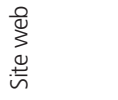 & 㟔 & 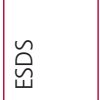 \\
\hline 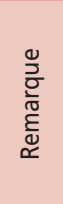 & 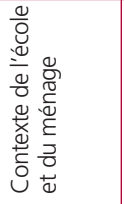 & 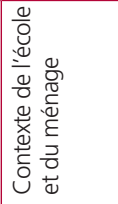 & 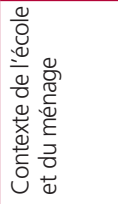 & & 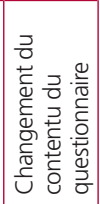 \\
\hline 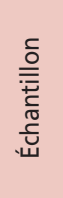 & 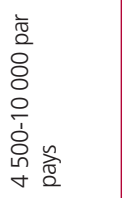 & 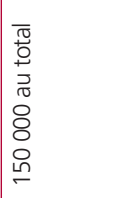 & 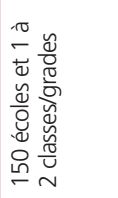 & 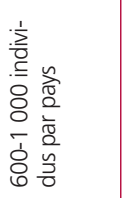 & 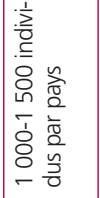 \\
\hline $\begin{array}{l}\frac{.0}{0} \\
\frac{\pi}{5} \\
\frac{0}{0} \\
0\end{array}$ & 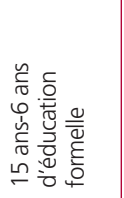 & $\begin{array}{l}\frac{n}{\pi} \\
\stackrel{0}{0} \\
\frac{0}{\sigma}\end{array}$ & 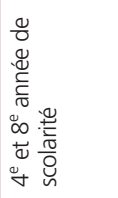 & 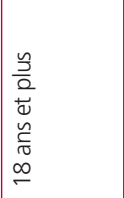 & 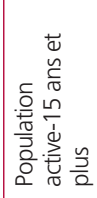 \\
\hline 节 & 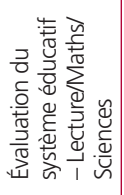 & 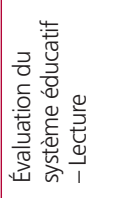 & 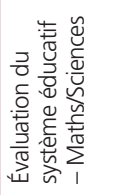 & 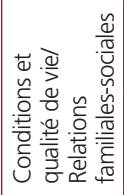 & 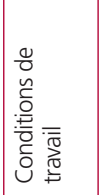 \\
\hline 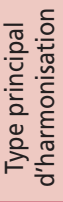 & 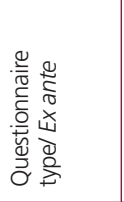 & 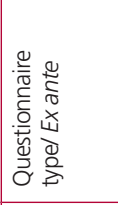 & 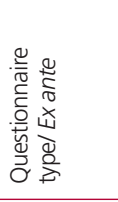 & 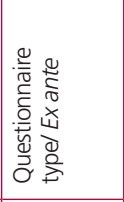 & 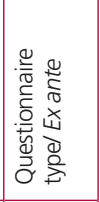 \\
\hline ๙ֶ๊ & 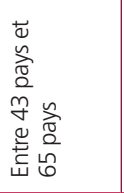 & 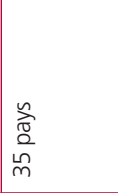 & 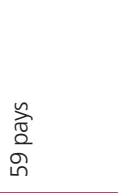 & 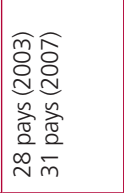 & 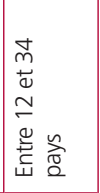 \\
\hline 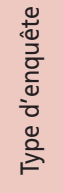 & 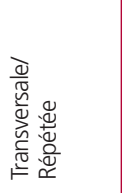 & 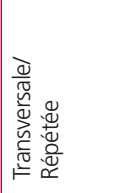 & 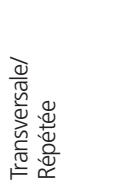 & 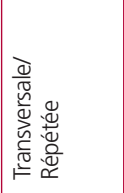 & 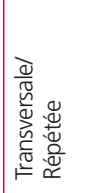 \\
\hline 离 & 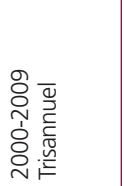 & 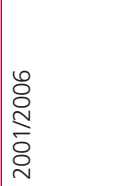 & 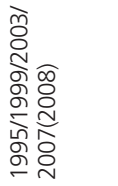 & 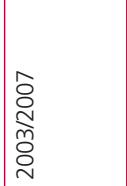 & 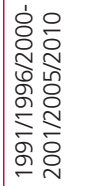 \\
\hline $\begin{array}{l}\varepsilon \\
\text { ह }\end{array}$ & 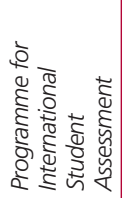 & 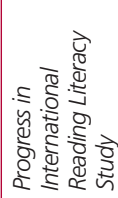 & 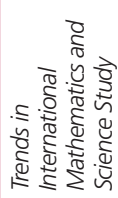 & 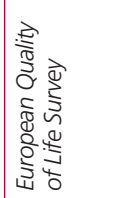 & 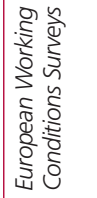 \\
\hline$\frac{0}{5}$ & $\frac{\pi}{\alpha}$ & $\frac{\overrightarrow{\underline{\alpha}}}{\alpha}$ & $\sum_{\equiv}^{n}$ & 岂 & 兑 \\
\hline
\end{tabular}


Determinants of Health (ESMD) et le European Survey Module on Care (ESMC) qui devraient rendre les données collectées comparables sont présentés sur le site de la Commission européenne ${ }^{(92)}$. Cette initiative a, par exemple, influencé le choix des questions posées dans EU-SILC. La question qui figure dans quasiment toutes les enquêtes concerne la santé générale, question dont la comparabilité dépend entre autres du choix des catégories de réponse. D’autres initiatives pour rendre les données concernant la santé et les incapacités comparables sont entreprises par l'Organisation mondiale de la santé( ${ }^{(93)}$ et le Washington Group on Disability Statistics ${ }^{(94)}$, une initiative de la Division de statistiques des Nations unies.

Concernant la vaste thématique des migrations, les enquêtes rencontrent en plus des problèmes de comparabilité, des limitations à cause des faibles effectifs des sous-populations étudiées. Le projet européen PROMINSTAT(95) a fait un état des lieux des différentes sources de données quantitatives disponibles dans les 27 pays de l'Union européenne et a créé une base de données regroupant les résultats. Une des enquêtes les plus importantes pour l'analyse des migrations est l'Enquête sur les forces de travail (EFT). Mais les deux panels européens, l'enquête PISA et d'autres enquêtes permettent eux aussi une analyse, quoique limitée, des migrations et des caractéristiques des migrants. Une enquête spécifique, EU-MIDIS(96), se concentre sur les discriminations vécues par les migrants et les minorités nationales dans les 27 pays de l'UE. Elle a été réalisée par l'Agence des droits fondamentaux de l'Union européenne (FRA) et une attention particulière a été accordée à la comparabilité entre pays.

Un effort important a été fourni par l'UNECE dans les années 1990 de façon à mettre en route une enquête comparative en Europe sur la famille et la fécondité (Family and Fertility Survey, FFS) ${ }^{(97)}$ et une documentation très riche est disponible sur le site web. Pour donner une suite à cette enquête, et encore une fois sur l'initiative de l'UNECE, a été créé le Generation and Gender Programme autour d'une enquête sous forme de panel, la Generation and Gender Survey (GGS) ${ }^{(98)}$. Ces deux enquêtes permettent d'analyser la structure des familles (traditionnelles ou avec les nouvelles formes de cohabitation dans la GGS) et son évolution dans une perspective comparative à travers l'espace, ainsi que dans le temps pour la GGS. Mais alors que la FFS couvrait un vaste nombre de pays, la couverture géographique n'est malheureusement pas encore très équilibrée. Dans beaucoup d'autres enquêtes, par contre, les liens familiaux sont ignorés ou traités d'une manière sommaire, ce qui ne permet pas de les

\footnotetext{
(92) http://ec.europa.eu/health/data_collection/tools/mechanisms/index_en.htm\#fragment0

(93) http://www.who.int/healthinfo/survey/en/index.html

(94) http://www.cdc.gov/nchs/citygroup.htm

(95) http://www.prominstat.eu

(96) http://fra.europa.eu/fraWebsite/research/projects/proj_eumidis_en.htm

(97) http://www.unece.org/pau/ffs/ffs.htm

(98) http://www.unece.org/pau/ggp/Welcome.html
} 
analyser de façon satisfaisante. Il est nécessaire de connaître non seulement le nombre d'enfants vivants, mais aussi celui des enfants décédés, question rarement posée. De même, il serait plus intéressant de prendre en compte à la fois les enfants vivant dans le même ménage et ceux qui ont déjà quitté le foyer parental. SHARE peut être cité comme exemple de tentative pour ne pas se limiter au ménage, l'analyse de la fréquence et du type de contact allant même au-delà de la famille, incluant les voisins et les amis.

\section{Quelques réflexions en conclusion}

D'énormes efforts ont été fournis ces dernières décennies pour rendre les données d'enquêtes plus comparables et accessibles à travers l'Europe et au-delà. Néanmoins, les différentes initiatives restent souvent difficiles à identifier. Un inventaire systématique et régulièrement mis à jour de ces efforts aidera à renforcer la coopération entre les différents projets et à éviter des répétitions inutiles.

De multiples essais ont été effectués pour homogénéiser les méthodes de collecte et en particulier d'échantillonnage. L'hétérogénéité des cadres d'échantillonnage pour une même enquête comparative introduit un biais mal maîtrisé. On trouve parfois de fortes variations dans les tailles des échantillons entre pays pour une même enquête, ce qui met en question la comparaison des résultats, leur significativité étant directement influencée par le nombre de personnes interrogées. De même, la taille parfois très faible des échantillons des enquêtes européennes ne permet pas toujours des analyses approfondies des comportements démographiques ou sociaux. Dans la perspective actuelle, hormis l'âge et sexe, il faut souvent contrôler par l'état matrimonial, le niveau d'éducation et la santé. Avec un petit échantillon, les limites du possible sont alors vite atteintes. 

ANNEXE STATISTIQUE 
Tableau A.1. Population au $1^{\text {er }}$ janvier (milliers)

\begin{tabular}{|c|c|c|c|c|c|c|c|}
\hline & 1980 & 1990 & 2000 & 2007 & 2008 & 2009 & 2010 \\
\hline \multicolumn{8}{|c|}{ Europe du Nord } \\
\hline Danemark & 5120 & 5135 & 5330 & 5447 & 5476 & 5511 & 5535 \\
\hline Finlande & 4771 & 4974 & 5171 & 5277 & 5300 & 5326 & 5351 \\
\hline Islande & 227 & 254 & 279 & 308 & 315 & 319 & 318 \\
\hline Norvège & 4079 & 4233 & 4478 & 4681 & 4737 & 4799 & 4855 \\
\hline Suède & 8303 & 8527 & 8861 & 9113 & 9183 & 9256 & 9341 \\
\hline \multicolumn{8}{|c|}{ Europe de l'Ouest } \\
\hline Allemagne & 78180 & 79113 & 82163 & 82315 & 82218 & 82002 & 81758 \\
\hline Autriche & 7546 & 7645 & 8002 & 8283 & 8319 & 8355 & 8375 \\
\hline Belgique & 9855 & 9948 & 10239 & 10585 & 10667 & 10750 & 10828 \\
\hline France & 53731 & 56577 & 58858 & 61795 & 62130 & 62469 & 62808 \\
\hline Irlande & 3393 & 3507 & 3778 & 4313 & 4401 & 4450 & 4451 \\
\hline Luxembourg & 363 & 379 & 436 & 476 & 484 & 493 & 502 \\
\hline Pays-Bas & 14091 & 14893 & 15864 & 16358 & 16404 & 16486 & 16578 \\
\hline Royaume-Uni & 56285 & 57157 & 58785 & 60781 & 61179 & 61635 & 62042 \\
\hline Suisse & 6304 & 6674 & 7164 & 7509 & 7593 & 7702 & 7760 \\
\hline \multicolumn{8}{|c|}{ Europe du Sud } \\
\hline Albanie & 2645 & 3143 & 3058 & 3153 & 3170 & 3194 & 3195 \\
\hline $\begin{array}{l}\text { Bosnie- } \\
\text { Herzégovine }\end{array}$ & 4137 & 4499 & 3781 & 3844 & 3844 & 3844 & 3844 \\
\hline Chypre & 510 & 573 & 690 & 779 & 789 & 797 & 802 \\
\hline Croatie & 4598 & 4688 & 4568 & 4441 & 4436 & 4435 & 4426 \\
\hline Espagne & 37242 & 38826 & 39961 & 44475 & 45283 & 45828 & 46087 \\
\hline Grèce & 9588 & 10121 & 10904 & 11172 & 11214 & 11260 & 11306 \\
\hline Italie & 56388 & 56694 & 57680 & 59131 & 59619 & 60045 & 60397 \\
\hline Macédoine & 1878 & 1873 & 2022 & 2042 & 2045 & 2049 & 2053 \\
\hline Malte & 323 & 352 & 380 & 408 & 410 & 414 & 416 \\
\hline Monténégro & 584 & 642 & 612 & 625 & 628 & 630 & 633 \\
\hline Portugal & 9714 & 9920 & 10195 & 10599 & 10618 & 10627 & 10637 \\
\hline Serbie & 9185 & 9859 & 7528 & 7398 & 7366 & 7335 & 7307 \\
\hline Slovénie & 1893 & 1996 & 1988 & 2010 & 2010 & 2032 & 2054 \\
\hline \multicolumn{8}{|c|}{ Europe centrale } \\
\hline Bulgarie & 8846 & 8767 & 8191 & 7679 & 7640 & 7607 & 7577 \\
\hline Hongrie & 10709 & 10375 & 10222 & 10066 & 10045 & 10031 & 10013 \\
\hline Pologne & 35413 & 38038 & 38263 & 38125 & 38116 & 38136 & 38164 \\
\hline Rép. tchèque & 10273 & 10362 & 10278 & 10287 & 10381 & 10468 & 10507 \\
\hline Roumanie & 22133 & 23211 & 22455 & 21565 & 21529 & 21499 & 21466 \\
\hline Slovaquie & 4963 & 5288 & 5399 & 5394 & 5401 & 5412 & 5425 \\
\hline \multicolumn{8}{|c|}{ Europe de l'Est } \\
\hline Belarus & 9592 & 10189 & 10019 & 9714 & 9690 & 9672 & 9480 \\
\hline Estonie & 1472 & 1571 & 1372 & 1342 & 1341 & 1340 & 1340 \\
\hline Lettonie & 2509 & 2668 & 2382 & 2281 & 2271 & 2261 & 2249 \\
\hline Lituanie & 3404 & 3694 & 3512 & 3385 & 3366 & 3350 & 3329 \\
\hline Moldavie & 3987 & 4362 & 3643 & 3581 & 3573 & 3568 & 3564 \\
\hline Russie & 138127 & 147665 & 146890 & 142221 & 142009 & 141904 & 141915 \\
\hline Ukraine & 49952 & 51838 & 49430 & 46466 & 46192 & 45964 & 45783 \\
\hline
\end{tabular}


Tableau A.2. Taux d'accroissement naturel (pour 1 000)

\begin{tabular}{|c|c|c|c|c|c|c|}
\hline & 1980 & 1990 & 2000 & 2007 & 2008 & 2009 \\
\hline \multicolumn{7}{|c|}{ Europe du Nord } \\
\hline Danemark & 0,3 & 0,5 & 1,7 & 1,6 & 1,9 & 1,4 \\
\hline Finlande & 3,9 & 3,1 & 1,4 & 1,8 & 2,0 & 2,1 \\
\hline Islande & 13,1 & 12,0 & 8,9 & 8,4 & 8,9 & 9,3 \\
\hline Norvège & 2,4 & 3,5 & 3,4 & 3,5 & 3,9 & 4,1 \\
\hline Suède & 0,6 & 3,4 & $-0,3$ & 1,7 & 1,9 & 2,3 \\
\hline \multicolumn{7}{|c|}{ Europe de l'Ouest } \\
\hline Allemagne & $-1,1$ & $-0,2$ & $-0,9$ & $-1,7$ & $-2,0$ & $-2,3$ \\
\hline Autriche & $-0,2$ & 1,0 & 0,2 & 0,2 & 0,3 & $-0,1$ \\
\hline Belgique & 1,1 & 2,0 & 1,0 & 1,9 & 2,2 & 2,1 \\
\hline France & 4,7 & 4,2 & 4,1 & 4,3 & 4,2 & 4,3 \\
\hline Irlande & 11,9 & 6,2 & 6,2 & 9,8 & 10,6 & 10,0 \\
\hline Luxembourg & 0,2 & 3,1 & 4,5 & 3,4 & 4,1 & 4,0 \\
\hline Pays-Bas & 4,7 & 4,6 & 4,2 & 3,0 & 3,0 & 3,1 \\
\hline Royaume-Uni & 1,7 & 2,7 & 1,2 & 3,2 & 3,5 & 3,6 \\
\hline Suisse & 2,3 & 3,0 & 2,2 & 1,8 & 2,0 & 2,1 \\
\hline \multicolumn{7}{|c|}{ Europe du Sud } \\
\hline Albanie & 20,1 & 19,5 & 11,0 & 5,9 & 6,3 & - \\
\hline $\begin{array}{l}\text { Bosnie- } \\
\text { Herzégovine }\end{array}$ & 10,9 & 8,4 & 2,4 & $-0,2$ & 0,0 & 0,9 \\
\hline Chypre & 11,1 & 9,7 & 4,5 & 4,1 & 5,1 & 5,1 \\
\hline Croatie & 3,9 & 0,7 & $-1,4$ & $-2,4$ & $-1,9$ & $-1,8$ \\
\hline Espagne & 7,6 & 1,8 & 0,9 & 2,5 & 2,9 & 3,9 \\
\hline Grèce & 6,3 & 0,8 & $-0,2$ & 0,2 & 0,9 & 0,9 \\
\hline Italie & 1,7 & 0,6 & $-0,3$ & $-0,1$ & $-0,1$ & $-0,5$ \\
\hline Macédoine & 13,9 & 11,0 & 6,0 & 1,5 & 1,9 & $-2,3$ \\
\hline Malte & 7,4 & 7,4 & 3,4 & 1,9 & 2,1 & 1,6 \\
\hline Monténégro & 11,7 & - & 6,1 & 3,0 & 4,1 & 4,4 \\
\hline Portugal & 6,5 & 1,3 & 1,4 & $-0,1$ & 0,0 & $-0,5$ \\
\hline Serbie & 8,4 & 5,3 & $-4,0$ & $-4,7$ & $-4,6$ & $-4,6$ \\
\hline Slovénie & 5,8 & 1,9 & $-0,2$ & 0,6 & 1,7 & 1,9 \\
\hline \multicolumn{7}{|c|}{ Europe centrale } \\
\hline Bulgarie & 3,4 & $-0,4$ & $-5,1$ & $-4,9$ & $-4,3$ & $-3,8$ \\
\hline Hongrie & 0,3 & $-1,9$ & $-3,7$ & $-3,5$ & $-3,1$ & $-3,4$ \\
\hline Pologne & 9,0 & 3,8 & $-0,2$ & $-0,3$ & 0,5 & 0,7 \\
\hline Rép. tchèque & 1,8 & 0,1 & $-1,8$ & 1,0 & 1,4 & 1,0 \\
\hline Roumanie & 7,5 & 2,9 & $-1,0$ & $-1,7$ & $-1,5$ & $-1,4$ \\
\hline Slovaquie & 8,9 & 4,8 & 0,4 & 0,1 & 0,8 & 1,5 \\
\hline \multicolumn{7}{|c|}{ Europe de l'Est } \\
\hline Belarus & 6,1 & 3,2 & $-4,1$ & $-3,0$ & $-2,7$ & - \\
\hline Estonie & 2,7 & 1,8 & $-3,9$ & $-1,2$ & $-0,5$ & $-0,2$ \\
\hline Lettonie & 1,4 & 1,2 & $-5,0$ & $-4,3$ & $-3,1$ & $-3,4$ \\
\hline Lituanie & 4,7 & 4,6 & $-1,4$ & $-3,9$ & $-2,6$ & $-1,6$ \\
\hline Moldavie & 9,8 & 7,9 & $-1,2$ & $-1,4$ & $-0,8$ & $-0,4$ \\
\hline Russie & 4,9 & 2,3 & $-6,5$ & $-3,3$ & $-2,6$ & $-1,8$ \\
\hline Ukraine & 3,5 & 0,5 & $-7,6$ & $-6,3$ & $-5,3$ & $-4,2$ \\
\hline
\end{tabular}


A. Avdeev et al.

Tableau A.3. Taux d'accroissement migratoire (pour 1000 )

\begin{tabular}{|c|c|c|c|c|c|c|}
\hline & 1980 & 1990 & 2000 & 2007 & 2008 & 2009 \\
\hline \multicolumn{7}{|c|}{ Europe du Nord } \\
\hline Danemark & 0,2 & 1,7 & 1,9 & 4,2 & 5,3 & 2,8 \\
\hline Finlande & $-0,5$ & 1,7 & 0,5 & 2,6 & 2,9 & 2,7 \\
\hline Islande & $-2,4$ & $-2,7$ & 6,1 & 16,5 & 3,6 & $-15,2$ \\
\hline Norvège & 0,9 & 0,4 & 2,2 & 8,4 & 9,1 & 7,5 \\
\hline Suède & 1,2 & 4,1 & 2,8 & 5,9 & 6,0 & 6,7 \\
\hline \multicolumn{7}{|c|}{ Europe de l'Ouest } \\
\hline Allemagne & 3,9 & 8,3 & 2,0 & 0,6 & $-0,7$ & $-0,7$ \\
\hline Autriche & 1,2 & 7,6 & 2,2 & 4,1 & 4,1 & 2,5 \\
\hline Belgique & $-0,3$ & 2,0 & 1,4 & 5,5 & 5,6 & 5,1 \\
\hline France & 0,8 & 1,4 & 1,2 & 1,1 & 1,2 & 1,1 \\
\hline Irlande & $-0,2$ & $-2,2$ & 8,3 & 10,6 & 0,4 & $-9,9$ \\
\hline Luxembourg & 3,7 & 10,3 & $-1,0$ & 12,5 & 15,8 & 13,2 \\
\hline Pays-Bas & 3,6 & 3,2 & 3,4 & $-0,1$ & 2,0 & 2,2 \\
\hline Royaume-Uni & $-0,1$ & 0,6 & 3,6 & 3,3 & 3,9 & 3,0 \\
\hline Suisse & 2,7 & 7,5 & 2,8 & 9,8 & 12,1 & 5,5 \\
\hline \multicolumn{7}{|c|}{ Europe du Sud } \\
\hline Albanie & 0,0 & $-27,7$ & $-5,5$ & $-0,4$ & $-1,2$ & - \\
\hline $\begin{array}{l}\text { Bosnie- } \\
\text { Herzégovine }\end{array}$ & $-16,4$ & $-4,3$ & 2,1 & 0,0 & 0,0 & 1,1 \\
\hline Chypre & 1,3 & 15,0 & 5,7 & 9,4 & 4,5 & 1,2 \\
\hline Croatie & $-3,4$ & $-1,1$ & $-27,5$ & 1,3 & 1,6 & $-1,3$ \\
\hline Espagne & 3,0 & $-0,5$ & 11,9 & 15,5 & 9,1 & 1,7 \\
\hline Grèce & 5,2 & 6,3 & 2,7 & 3,6 & 3,2 & 3,2 \\
\hline Italie & $-0,1$ & 0,2 & 3,2 & 8,3 & 7,3 & 6,4 \\
\hline Macédoine & 0,1 & $-1,6$ & $-1,2$ & 0,1 & $-0,3$ & $-0,3$ \\
\hline Malte & 2,5 & 2,4 & 25,7 & 4,2 & 5,9 & 5,0 \\
\hline Monténégro & $-14,5$ & - & $-2,4$ & 1,2 & 0,1 & 0,0 \\
\hline Portugal & 4,3 & $-5,6$ & 4,6 & 1,8 & 0,9 & 1,4 \\
\hline Serbie & 3,7 & $-7,3$ & 0,9 & 0,3 & 0,4 & 0,7 \\
\hline Slovénie & 2,9 & $-0,1$ & 1,4 & $-0,7$ & 9,2 & 8,8 \\
\hline \multicolumn{7}{|c|}{ Europe centrale } \\
\hline Bulgarie & 0,0 & $-10,9$ & 0,0 & $-0,2$ & $-0,1$ & $-0,1$ \\
\hline Hongrie & $-0,7$ & 1,8 & 1,6 & 1,4 & 1,6 & 1,6 \\
\hline Pologne & $-0,6$ & $-0,3$ & $-0,5$ & $-0,5$ & $-0,4$ & $-0,4$ \\
\hline Rép. tchèque & 0,2 & 0,1 & 0,6 & 8,1 & 6,9 & 2,7 \\
\hline Roumanie & $-0,8$ & $-3,7$ & $-0,2$ & 0,0 & 0,1 & $-0,1$ \\
\hline Slovaquie & $-0,6$ & $-0,4$ & 0,3 & 1,3 & 1,3 & 0,8 \\
\hline \multicolumn{7}{|c|}{ Europe de l'Est } \\
\hline Belarus & 1,3 & $-3,1$ & 1,2 & 0,5 & 0,8 & - \\
\hline Estonie & 4,1 & $-3,6$ & 0,2 & 0,1 & 0,1 & 0,1 \\
\hline Lettonie & 1,0 & $-4,9$ & $-2,3$ & $-0,3$ & $-1,1$ & $-2,1$ \\
\hline Lituanie & 0,6 & $-2,4$ & $-5,8$ & $-1,6$ & $-2,3$ & $-4,6$ \\
\hline Moldavie & 1,4 & $-6,9$ & $-1,5$ & $-0,9$ & $-0,6$ & $-0,7$ \\
\hline Russie & 0,3 & 1,1 & 1,5 & 1,8 & 1,8 & 1,8 \\
\hline Ukraine & 0,2 & 1,5 & $-8,0$ & 0,4 & 0,3 & 0,3 \\
\hline
\end{tabular}


Tableau A.4. Indicateur conjoncturel de fécondité (enfants par femme)

\begin{tabular}{|c|c|c|c|c|c|c|}
\hline & 1980 & 1990 & 2000 & 2007 & 2008 & 2009 \\
\hline \multicolumn{7}{|c|}{ Europe du Nord } \\
\hline Danemark & 1,55 & 1,67 & 1,77 & 1,85 & 1,89 & 1,84 \\
\hline Finlande & 1,63 & 1,78 & 1,73 & 1,83 & 1,85 & 1,86 \\
\hline Islande & 2,48 & 2,31 & 2,08 & 2,09 & 2,14 & 2,23 \\
\hline Norvège & 1,72 & 1,89 & 1,85 & 1,90 & 1,90 & 1,96 \\
\hline Suède & 1,68 & 2,14 & 1,55 & 1,88 & 1,91 & 1,94 \\
\hline \multicolumn{7}{|c|}{ Europe de l'Ouest } \\
\hline Allemagne & 1,56 & 1,45 & 1,38 & 1,37 & 1,38 & 1,36 \\
\hline Autriche & 1,65 & 1,46 & 1,36 & 1,38 & 1,41 & 1,39 \\
\hline Belgique & 1,68 & 1,62 & 1,62 & 1,81 & 1,86 & 1,84 \\
\hline France & 1,95 & 1,78 & 1,88 & 1,96 & 1,99 & 1,99 \\
\hline Irlande & 3,24 & 2,12 & 1,90 & 2,03 & 2,10 & 2,07 \\
\hline Luxembourg & 1,49 & 1,62 & 1,78 & 1,61 & 1,61 & 1,59 \\
\hline Pays-Bas & 1,60 & 1,62 & 1,72 & 1,72 & 1,77 & 1,79 \\
\hline Royaume-Uni & 1,89 & 1,83 & 1,64 & 1,90 & 1,94 & - \\
\hline Suisse & 1,55 & 1,59 & 1,50 & 1,45 & 1,48 & 1,50 \\
\hline \multicolumn{7}{|c|}{ Europe du Sud } \\
\hline Albanie & 3,62 & 3,03 & 2,00 & 1,33 & 1,40 & - \\
\hline $\begin{array}{l}\text { Bosnie- } \\
\text { Herzégovine }\end{array}$ & 1,93 & 1,71 & 1,30 & 1,18 & 1,19 & - \\
\hline Chypre & $2,48^{*}$ & 2,41 & 1,64 & 1,39 & 1,46 & 1,51 \\
\hline Croatie & 1,92 & 1,67 & 1,39 & 1,40 & 1,47 & 1,49 \\
\hline Espagne & 2,20 & 1,36 & 1,23 & 1,40 & 1,46 & 1,40 \\
\hline Grèce & 2,23 & 1,39 & 1,27 & 1,42 & 1,51 & 1,52 \\
\hline Italie & 1,64 & 1,36 & 1,26 & 1,37 & 1,42 & 1,41 \\
\hline Macédoine & 2,47 & 2,06 & 1,88 & 1,46 & 1,47 & - \\
\hline Malte & 1,99 & 2,05 & 1,69 & 1,37 & 1,43 & 1,44 \\
\hline Monténégro & 2,15 & 1,84 & 1,85 & 1,69 & 1,77 & 1,85 \\
\hline Portugal & 2,25 & 1,57 & 1,56 & 1,34 & 1,37 & 1,32 \\
\hline Serbie & 2,26 & 2,11 & 1,46 & 1,38 & 1,41 & 1,44 \\
\hline Slovénie & 2,11 & 1,46 & 1,26 & 1,38 & 1,53 & 1,53 \\
\hline \multicolumn{7}{|c|}{ Europe centrale } \\
\hline Bulgarie & 2,05 & 1,82 & 1,30 & 1,42 & 1,48 & 1,57 \\
\hline Hongrie & 1,91 & 1,87 & 1,32 & 1,32 & 1,35 & 1,33 \\
\hline Pologne & 2,26 & 1,99 & 1,37 & 1,31 & 1,39 & 1,40 \\
\hline Rép. tchèque & 2,10 & 1,89 & 1,14 & 1,44 & 1,50 & 1,49 \\
\hline Roumanie & 2,43 & 1,84 & 1,31 & 1,29 & 1,35 & 1,38 \\
\hline Slovaquie & 2,31 & 2,08 & 1,29 & 1,25 & 1,32 & 1,41 \\
\hline \multicolumn{7}{|c|}{ Europe de l'Est } \\
\hline Belarus & 2,04 & 1,90 & 1,31 & 1,37 & 1,42 & - \\
\hline Estonie & 2,02 & 2,05 & 1,34 & 1,64 & 1,66 & 1,62 \\
\hline Lettonie & 1,90 & 2,01 & 1,24 & 1,42 & 1,44 & 1,31 \\
\hline Lituanie & 1,99 & 2,03 & 1,39 & 1,35 & 1,47 & 1,55 \\
\hline Moldavie & 2,41 & 2,39 & 1,29 & 1,26 & 1,28 & 1,33 \\
\hline Russie & 1,86 & 1,89 & 1,20 & 1,41 & 1,49 & 1,54 \\
\hline Ukraine & 1,95 & 1,89 & 1,09 & 1,30 & 1,39 & - \\
\hline
\end{tabular}


A. Avdeev et al.

Tableau A.5. Proportion de naissances hors mariage (pour 100 naissances vivantes)

\begin{tabular}{|c|c|c|c|c|c|c|}
\hline & 1980 & 1990 & 2000 & 2007 & 2008 & 2009 \\
\hline \multicolumn{7}{|c|}{ Europe du Nord } \\
\hline Danemark & 33,2 & 46,4 & 44,6 & 46,1 & 46,2 & 46,6 \\
\hline Finlande & 13,1 & 25,2 & 39,2 & 40,6 & 40,7 & - \\
\hline Islande & 39,7 & 55,2 & 65,2 & 63,8 & 64,1 & - \\
\hline Norvège & 14,5 & 38,6 & 49,6 & 54,5 & 55,8 & - \\
\hline Suède & 39,7 & 47,0 & 55,3 & 54,8 & 54,7 & - \\
\hline \multicolumn{7}{|c|}{ Europe de l'Ouest } \\
\hline Allemagne & 11,9 & 15,3 & 23,4 & 30,8 & 32,1 & - \\
\hline Autriche & 17,8 & 23,6 & 31,3 & 38,2 & 38,8 & 39,3 \\
\hline Belgique & 4,1 & 11,6 & $25,6^{*}$ & 39,0 & - & - \\
\hline France & 11,4 & 30,1 & 42,6 & 50,7 & 51,6 & - \\
\hline Irlande & 50,3 & 14,6 & 31,5 & 32,8 & 33,1 & 32,8 \\
\hline Luxembourg & 6,0 & 12,9 & 21,9 & 30,7 & 30,2 & - \\
\hline Pays-Bas & 4,1 & 11,4 & 24,9 & 39,5 & 41,2 & 41,2 \\
\hline Royaume-Uni & 11,5 & 27,9 & 39,5 & $43,7^{* *}$ & - & - \\
\hline Suisse & 4,7 & 6,1 & 10,7 & 16,2 & 17,1 & - \\
\hline \multicolumn{7}{|c|}{ Europe du Sud } \\
\hline Albanie & - & - & - & - & - & - \\
\hline $\begin{array}{l}\text { Bosnie- } \\
\text { Herzégovine }\end{array}$ & 5,4 & 7,4 & 10,3 & 11,5 & 10,8 & 10,5 \\
\hline Chypre & 0,6 & 0,7 & 2,3 & 8,7 & 8,9 & - \\
\hline Croatie & 5,1 & 7,0 & 9,0 & 11,5 & 12,0 & 12,9 \\
\hline Espagne & 3,9 & 9,6 & 17,7 & 30,2 & 33,2 & - \\
\hline Grèce & 1,5 & 2,2 & 4,0 & 5,8 & 5,9 & - \\
\hline Italie & 4,2 & 6,3 & 9,7 & 20,7 & 20,8 & - \\
\hline Macédoine & 6,1 & 7,1 & 9,8 & 12,6 & 12,2 & 12,2 \\
\hline Malte & 1,1 & 1,8 & 10,9 & 24,9 & 25,4 & - \\
\hline Monténégro & 3,7 & 6,6 & - & 15,6 & 17,4 & 15,7 \\
\hline Portugal & 9,2 & 14,7 & 22,2 & 33,6 & 36,2 & - \\
\hline Serbie & $10,1 * * *$ & $12,7 * * *$ & 20,7 & 22,3 & 22,8 & - \\
\hline Slovénie & 13,1 & 24,5 & 37,1 & 50,8 & 52,8 & - \\
\hline \multicolumn{7}{|c|}{ Europe centrale } \\
\hline Bulgarie & 10,9 & 12,4 & 38,4 & 50,2 & 51,1 & - \\
\hline Hongrie & 7,1 & 13,1 & 29,0 & 37,5 & 39,5 & 40,8 \\
\hline Pologne & 4,7 & 6,2 & 12,1 & 19,5 & 19,9 & - \\
\hline Rép. tchèque & 5,7 & 8,6 & 21,9 & 34,6 & 36,3 & 38,8 \\
\hline Roumanie & 2,8 & 4,0 & 25,5 & 26,7 & 27,4 & - \\
\hline Slovaquie & 5,8 & 7,7 & 18,3 & 28,8 & 29,9 & 29,9 \\
\hline \multicolumn{7}{|c|}{ Europe de l'Est } \\
\hline Belarus & 6,4 & 8,5 & 18,6 & 21,2 & 20,1 & - \\
\hline Estonie & 18,3 & 27,2 & 54,5 & 58,1 & 59,1 & - \\
\hline Lettonie & 12,5 & 16,9 & 40,3 & 43,0 & 43,1 & - \\
\hline Lituanie & 6,3 & 7,0 & 22,6 & 29,2 & 28,6 & - \\
\hline Moldavie & 7,4 & 11,1 & 20,5 & 22,7 & 22,3 & 22,9 \\
\hline Russie & 10,8 & 14,6 & 28,0 & 28,0 & 26,9 & - \\
\hline Ukraine & 8,8 & 13,0 & 17,3 & 21,4 & 20,9 & - \\
\hline
\end{tabular}


Tabeau A.6. Espérance de vie à la naissance (années)

\begin{tabular}{|c|c|c|c|c|c|c|c|c|c|c|}
\hline & \multicolumn{2}{|c|}{1980} & \multicolumn{2}{|c|}{1990} & \multicolumn{2}{|c|}{2000} & \multicolumn{2}{|c|}{2007} & \multicolumn{2}{|c|}{2008} \\
\hline & $\mathrm{H}$ & $\mathrm{F}$ & $\mathrm{H}$ & $\mathrm{F}$ & $\mathrm{H}$ & $\mathrm{F}$ & $\mathrm{H}$ & $\mathrm{F}$ & $\mathrm{H}$ & $\mathrm{F}$ \\
\hline \multicolumn{11}{|c|}{ Europe du Nord } \\
\hline Danemark & 71,2 & 77,2 & 72,0 & 77,8 & 74,5 & 79,3 & 76,1 & 80,5 & 76,5 & 80,9 \\
\hline Finlande & 69,3 & 78,0 & 71,0 & 79,0 & 74,2 & 81,2 & 76,0 & 83,1 & 76,5 & 83,3 \\
\hline Islande & 73,8 & 80,3 & 75,7 & 81,1 & 78,0 & 81,4 & 79,6 & 83,4 & 80,0 & 83,3 \\
\hline Norvège & 72,3 & 79,1 & 73,4 & 79,8 & 76,0 & 81,4 & 78,2 & 82,7 & 78,3 & 83,0 \\
\hline Suède & 72,8 & 78,9 & 74,8 & 80,4 & 77,4 & 82,0 & 78,9 & 83,0 & 79,1 & 83,2 \\
\hline \multicolumn{11}{|c|}{ Europe de l'Ouest } \\
\hline Allemagne & 69,6 & 76,1 & 72,0 & 78,4 & 75,0 & 81,0 & 76,9 & 82,3 & 77,2 & 82,4 \\
\hline Autriche & 69,0 & 76,0 & 72,2 & 78,8 & 75,1 & 81,1 & 77,3 & 82,8 & 77,6 & 83,0 \\
\hline Belgique & 69,9 & 76,7 & 72,7 & 79,5 & 74,6 & 81,0 & 77,3 & 83,3 & 77,5 & 83,5 \\
\hline France & 70,2 & 78,4 & 72,7 & 81,0 & 75,3 & 82,8 & 77,6 & 84,5 & 77,6 & 84,4 \\
\hline Irlande* & 69,8 & 75,1 & 71,9 & 77,6 & 73,8 & 79,1 & 77,2 & 81,9 & 77,9 & 82,5 \\
\hline Luxembourg & 70,0 & 75,6 & 72,4 & 78,7 & 74,6 & 81,3 & 76,7 & 82,2 & 78,1 & 83,1 \\
\hline Pays-Bas & 72,4 & 79,1 & 73,8 & 80,1 & 75,5 & 80,6 & 78,1 & 82,3 & 78,3 & 82,3 \\
\hline $\begin{array}{l}\text { Royaume- } \\
\text { Uni }\end{array}$ & 70,8 & 76,9 & 72,9 & 78,6 & 75,5 & 80,3 & 77,6 & 81,8 & - & - \\
\hline Suisse & 72,3 & 78,8 & 74,0 & 80,8 & 76,9 & 82,6 & 79,4 & 84,2 & 79,7 & 84,4 \\
\hline \multicolumn{11}{|c|}{ Europe du Sud } \\
\hline Albanie & 67,0 & 72,3 & 69,6 & 75,5 & 72,1 & 78,6 & $73,9 * *$ & $78,0 * *$ & 72,9 & 77,8 \\
\hline $\begin{array}{l}\text { Bosnie- } \\
\text { Herzégovine }\end{array}$ & 67,9 & 72,9 & 69,7 & 75,2 & 71,3 & 76,7 & 72,1 & 77,5 & 72,4 & 77,7 \\
\hline Chypre & - & - & 74,1 & 78,6 & 75,4 & 80,1 & 77,9 & 82,2 & 78,5 & 83,1 \\
\hline Croatie & 66,6 & 74,2 & 68,4 & 76,0 & 70,5 & 77,8 & 72,3 & 79,2 & 72,4 & 79,6 \\
\hline Espagne & 72,3 & 78,5 & 73,4 & 80,5 & 75,6 & 82,5 & 77,8 & 84,1 & 78,2 & 84,3 \\
\hline Grèce & 73,0 & 77,5 & 74,7 & 79,5 & 75,5 & 80,6 & 77,1 & 81,8 & 77,7 & 82,4 \\
\hline Italie & 70,7 & 77,4 & 73,6 & 80,1 & 76,5 & 82,5 & 78,7 & 84,0 & 78,6 & 84,0 \\
\hline Macédoine & 68,3 & 72,1 & 70,3 & 74,5 & 70,9 & 75,3 & 71,8 & 75,9 & 72,4 & 76,5 \\
\hline Malte & 68,7 & 72,8 & 73,7 & 78,1 & 74,3 & 80,2 & 77,5 & 82,2 & 77,1 & 82,3 \\
\hline Monténégro & 71,4 & 76,0 & $73,1 * * *$ & $78,2 * * *$ & 71,1 & 76,3 & 71,2 & 76,1 & 71,2 & 76,1 \\
\hline Portugal & 68,0 & 74,9 & 70,6 & 77,5 & 72,9 & 79,9 & 75,2 & 81,6 & 75,5 & 81,7 \\
\hline Serbie & 68,2 & 72,7 & $68,5^{* * *}$ & $73,6 * * *$ & 69,7 & 74,8 & 70,7 & 76,2 & 71,1 & 76,3 \\
\hline Slovénie & 67,3 & 75,2 & 69,8 & 77,8 & 72,2 & 79,9 & 74,7 & 82,0 & 75,5 & 82,6 \\
\hline \multicolumn{11}{|c|}{ Europe centrale } \\
\hline Bulgarie & 68,4 & 73,8 & 68,0 & 74,7 & 68,4 & 75,1 & 69,2 & 76,3 & 69,5 & 76,6 \\
\hline Hongrie & 65,5 & 72,7 & 65,2 & 73,7 & 67,1 & 75,6 & 69,2 & 77,3 & 69,8 & 77,8 \\
\hline Pologne & 66,0 & 74,4 & 66,2 & 75,2 & 69,7 & 78,0 & 71,0 & 79,7 & 71,3 & 80,0 \\
\hline $\begin{array}{l}\text { Rép. } \\
\text { tchèque }\end{array}$ & 66,8 & 73,9 & 67,6 & 75,4 & 71,7 & 78,4 & 73,7 & 79,9 & 74,0 & 80,1 \\
\hline Roumanie & 66,8 & 72,8 & 66,6 & 73,1 & 67,7 & 74,8 & 69,7 & 76,9 & 69,7 & 77,2 \\
\hline Slovaquie & 66,8 & 74,3 & 66,6 & 75,4 & 69,1 & 77,2 & 70,5 & 78,1 & 70,9 & 78,7 \\
\hline \multicolumn{11}{|c|}{ Europe de l'Est } \\
\hline Belarus & 65,9 & 75,5 & 66,3 & 75,6 & 63,4 & 74,6 & 64,5 & 76,2 & 64,7 & 76,5 \\
\hline Estonie & 64,2 & 74,2 & 64,7 & 74,9 & 65,6 & 76,4 & 67,1 & 78,7 & 68,6 & 79,2 \\
\hline Lettonie & 63,6 & 74,2 & 64,3 & 74,6 & 64,9 & 76,0 & 65,8 & 76,5 & 67,2 & 77,9 \\
\hline Lituanie & 65,4 & 75,4 & 66,4 & 76,2 & 66,8 & 77,4 & 64,9 & 77,2 & 66,3 & 77,6 \\
\hline Moldavie & 62,6 & 69,3 & 65,0 & 71,8 & 63,9 & 71,2 & 65,0 & 72,6 & 65,6 & 73,2 \\
\hline Russie & 61,5 & 73,0 & 63,7 & 74,3 & 59,0 & 72,3 & 61,4 & 73,9 & 61,8 & 74,2 \\
\hline Ukraine & 64,6 & 74,0 & 65,6 & 74,9 & 62,9 & 74,1 & 62,5 & 74,2 & 62,5 & 74,3 \\
\hline
\end{tabular}


A. Avdeev et al.

Tableau A.7. Taux de mortalité infantile (pour 1000)

\begin{tabular}{|c|c|c|c|c|}
\hline & 1980 & 1990 & 2000 & 2008 \\
\hline \multicolumn{5}{|l|}{ Europe du Nord } \\
\hline Danemark & 8,4 & 7,5 & 5,3 & 4,0 \\
\hline Finlande & 7,6 & 5,6 & 3,8 & 2,6 \\
\hline Islande & 7,7 & 5,9 & 3,0 & 2,5 \\
\hline Norvège & 8,1 & 6,9 & 3,8 & 2,7 \\
\hline Suède & 6,9 & 6,0 & 3,4 & 2,5 \\
\hline \multicolumn{5}{|l|}{ Europe de l'Ouest } \\
\hline Allemagne & 12,4 & 7,0 & 4,4 & 3,5 \\
\hline Autriche & 14,3 & 7,8 & 4,8 & 3,7 \\
\hline Belgique & 12,1 & 8,0 & 4,8 & 3,4 \\
\hline France & 10,0 & 7,3 & 4,4 & 3,6 \\
\hline Irlande & 11,1 & 8,2 & 6,2 & 3,9 \\
\hline Luxembourg & 11,5 & 7,3 & 5,1 & 1,8 \\
\hline Pays-Bas & 8,6 & 7,1 & 5,1 & 3,8 \\
\hline Royaume-Uni & 12,1 & 7,9 & 5,6 & 4,7 \\
\hline Suisse & 9,1 & 6,8 & 4,9 & 3,8 \\
\hline \multicolumn{5}{|l|}{ Europe du Sud } \\
\hline Albanie & 50,3 & 28,3 & 11,9 & 6,0 \\
\hline Bosnie-Herzégovine & 31,5 & 15,3 & 9,7 & 6,9 \\
\hline Chypre & - & 11,0 & 5,6 & 3,5 \\
\hline Croatie & 20,6 & 10,7 & 7,4 & 4,5 \\
\hline Espagne & 12,3 & 7,6 & 4,4 & 3,4 \\
\hline Grèce & 17,9 & 9,7 & 5,9 & 2,7 \\
\hline Italie & 14,2 & 8,0 & 4,5 & 3,6 \\
\hline Macédoine & 54,2 & 31,6 & 11,8 & 9,7 \\
\hline Malte & 15,2 & 9,1 & 5,9 & 8,2 \\
\hline Monténégro & 23,4 & - & 11,1 & 6,0 \\
\hline Portugal & 24,3 & 11,0 & 5,5 & 3,3 \\
\hline Serbie & 34,1 & 23,2 & 10,7 & 6,7 \\
\hline Slovénie & 15,3 & 8,4 & 4,9 & 2,4 \\
\hline \multicolumn{5}{|l|}{ Europe centrale } \\
\hline Bulgarie & 20,2 & 14,8 & 13,3 & 8,6 \\
\hline Hongrie & 23,2 & 14,8 & 9,2 & 5,6 \\
\hline Pologne & 25,4 & 19,4 & 8,1 & 5,6 \\
\hline Rép. tchèque & 16,9 & 10,8 & 4,1 & 2,8 \\
\hline Roumanie & 29,3 & 26,9 & 18,6 & 11,0 \\
\hline Slovaquie & 20,9 & 12,0 & 8,6 & 5,9 \\
\hline \multicolumn{5}{|l|}{ Europe de l'Est } \\
\hline Estonie & 17,1 & 12,3 & 8,4 & 5,0 \\
\hline Lettonie & 15,3 & 13,7 & 10,4 & 6,7 \\
\hline Lituanie & 14,5 & 10,2 & 8,6 & 4,9 \\
\hline Moldavie & 35,0 & 19,0 & 18,3 & 12,1 \\
\hline Russie & 22,0 & 17,6 & 15,2 & 8,5 \\
\hline États-Unis & 12,6 & 9,2 & 6,9 & - \\
\hline Canada & 10,4 & 6,8 & - & - \\
\hline
\end{tabular}


Tableau A.8. Probabilité de décéder entre 15 et 65 ans (pour 1000)

\begin{tabular}{|c|c|c|c|c|c|c|}
\hline & \multicolumn{2}{|c|}{1990} & \multicolumn{2}{|c|}{2000} & \multicolumn{2}{|c|}{2008} \\
\hline & $\mathrm{H}$ & $\mathrm{F}$ & $\mathrm{H}$ & $\mathrm{F}$ & $\mathrm{H}$ & $\mathrm{F}$ \\
\hline \multicolumn{7}{|l|}{ Europe du Nord } \\
\hline Danemark & 231,7 & 152,2 & 183,2 & 123,4 & 166,1 & 104,4 \\
\hline Finlande & 265,3 & 106,6 & 203,7 & 92,7 & 185,8 & 83,3 \\
\hline Islande & 165,3 & 108,6 & 133,6 & 90,8 & 103,5 & 73,6 \\
\hline Norvège & 198,2 & 103,4 & 156,2 & 93,9 & 125,6 & 81,0 \\
\hline Suède & 175,7 & 99,2 & 135,5 & 87,8 & 118,1 & 75,7 \\
\hline \multicolumn{7}{|l|}{ Europe de l'Ouest } \\
\hline Allemagne & 235,6 & 118,3 & 185,6 & 94,1 & 154,9 & 82,1 \\
\hline Autriche & 230,9 & 110,7 & 184,2 & 91,5 & 152,9 & 77,3 \\
\hline Belgique & 211,0 & 110,5 & 187,2 & 100,2 & 163,2 & 90,2 \\
\hline France & 231,0 & 95,8 & 196,2 & 86,7 & 169,9 & 78,9 \\
\hline Irlande & 220,0 & 129,6 & 181,5 & 108,3 & 135,0 & 86,2 \\
\hline Luxembourg & 233,2 & 119,4 & 181,1 & 95,7 & 159,0 & 87,8 \\
\hline Pays-Bas & 185,0 & 103,8 & 157,1 & 101,0 & 123,4 & 88,8 \\
\hline Royaume-Uni & 207,8 & 127,9 & 167,3 & 104,8 & 143,6 & 91,1 \\
\hline Suisse & 190,4 & 93,6 & 147,7 & 81,6 & 118,3 & 68,1 \\
\hline \multicolumn{7}{|l|}{ Europe du Sud } \\
\hline Albanie & 264,8 & 163,3 & 238,7 & 156,7 & 213,6 & 143,5 \\
\hline Bosnie-Herzégovine & 278,0 & 141,9 & 260,1 & 140,8 & 223,6 & 112,7 \\
\hline Chypre & 180,6 & 91,8 & 168,1 & 82,9 & 119,5 & 61,5 \\
\hline Croatie & 319,9 & 139,5 & 277,0 & 116,7 & 242,2 & 101,1 \\
\hline Espagne & 209,4 & 89,4 & 178,3 & 72,3 & 151,3 & 62,8 \\
\hline Grèce & 177,9 & 86,6 & 171,6 & 73,3 & 154,8 & 67,1 \\
\hline Italie & 199,8 & 93,6 & 153,9 & 77,2 & 122,5 & 64,7 \\
\hline Macédoine & 232,7 & 144,1 & 278,7 & 145,7 & 233,1 & 129,0 \\
\hline Malte & 233,2 & 110,3 & 181,1 & 87,2 & 159,0 & 72,0 \\
\hline Monténégro & 243,4 & 113,7 & 261,9 & 146,4 & 261,0 & 143,8 \\
\hline Portugal & 249,3 & 116,5 & 215,3 & 96,5 & 181,2 & 75,9 \\
\hline Serbie & 273,8 & 146,4 & 304,2 & 166,2 & 270,5 & 142,4 \\
\hline Slovénie & 294,4 & 123,2 & 244,1 & 109,2 & 193,9 & 83,6 \\
\hline \multicolumn{7}{|l|}{ Europe centrale } \\
\hline Bulgarie & 308,9 & 149,7 & 318,5 & 150,5 & 311,1 & 137,2 \\
\hline Hongrie & 413,8 & 193,6 & 374,7 & 166,9 & 332,6 & 150,1 \\
\hline Pologne & 366,6 & 156,4 & 309,8 & 130,0 & 289,2 & 118,1 \\
\hline Rép. tchèque & 342,0 & 151,6 & 258,7 & 117,3 & 221,1 & 102,0 \\
\hline Roumanie & 328,4 & 169,6 & 332,1 & 159,2 & 308,6 & 137,9 \\
\hline Slovaquie & 379,9 & 158,0 & 317,7 & 126,9 & 282,3 & 113,8 \\
\hline \multicolumn{7}{|l|}{ Europe de l'Est } \\
\hline Estonie & 406,9 & 164,6 & 424,2 & 166,0 & 348,8 & 127,8 \\
\hline Lettonie & 413,9 & 174,1 & 424,6 & 166,2 & 424,4 & 165,6 \\
\hline Lituanie & 380,7 & 158,1 & 385,7 & 146,7 & 416,8 & 160,5 \\
\hline Moldavie & 390,4 & 228,7 & 423,2 & 237,5 & 406,0 & 207,2 \\
\hline Russie & 424,9 & 174,6 & 557,4 & 225,0 & 395,9 & 202,2 \\
\hline États-Unis & 247,6 & 138,3 & 207,3 & 126,9 & 190,4 & 117,7 \\
\hline Canada & 201,2 & 109,2 & 154,2 & 93,6 & 131,3 & 82,2 \\
\hline
\end{tabular}


Tableau A.9. Espérance de vie à 65 ans et espérance de vie en bonne santé (EVBS) à 65 ans, en années

\begin{tabular}{|c|c|c|c|c|c|c|c|c|c|c|}
\hline & \multicolumn{8}{|c|}{ Espérance de vie à 65 ans } & \multirow{2}{*}{\multicolumn{2}{|c|}{$\begin{array}{l}\text { EVBS } \\
2007\end{array}$}} \\
\hline & \multicolumn{2}{|c|}{1980} & \multicolumn{2}{|c|}{1990} & \multicolumn{2}{|c|}{2000} & \multicolumn{2}{|c|}{2008} & & \\
\hline & $\mathrm{H}$ & $\mathrm{F}$ & $\mathrm{H}$ & $\mathrm{F}$ & $\mathrm{H}$ & $\mathrm{F}$ & $\mathrm{H}$ & $\mathrm{F}$ & $\mathrm{H}$ & $\mathrm{F}$ \\
\hline \multicolumn{11}{|l|}{ Europe du Nord } \\
\hline Danemark & 13,6 & 17,7 & 14,0 & 17,9 & 15,2 & 18,3 & 16,6 & 19,5 & 13,1 & 14,3 \\
\hline Finlande & 12,7 & 17,0 & 13,8 & 17,9 & 15,6 & 19,6 & 17,5 & 21,3 & 7,8 & 8,8 \\
\hline Islande & 15,7 & 19,3 & 16,4 & 19,8 & 17,8 & 19,8 & 18,4 & 20,6 & 15,7 & 16,5 \\
\hline Norvège & 14,3 & 18,2 & 14,6 & 18,7 & 16,1 & 19,9 & 17,6 & 21,0 & 12,2 & 13,1 \\
\hline Suède & 14,3 & 18,1 & 15,4 & 19,2 & 16,8 & 20,2 & 18,0 & 20,9 & 12,8 & 13,8 \\
\hline \multicolumn{11}{|l|}{ Europe de l'Ouest } \\
\hline Allemagne & 12,8 & 16,3 & 14,0 & 17,7 & 15,8 & 19,6 & 17,5 & 20,7 & 7,7 & 7,6 \\
\hline Autriche & 12,9 & 16,3 & 14,4 & 18,1 & 16,0 & 19,6 & 17,7 & 21,1 & 7,3 & 7,7 \\
\hline Belgique & 12,9 & 16,8 & 14,3 & 18,8 & 15,6 & 19,8 & 17,3 & 20,9 & 10,1 & 10,3 \\
\hline France & 14,0 & 18,2 & 15,7 & 20,2 & 16,8 & 21,4 & 18,5 & 23,0 & 9,4 & 9,9 \\
\hline Irlande & 12,6 & 15,6 & 13,2 & 17,1 & 14,6 & 18,0 & 16,8 & 20,3 & 9,6 & 10,4 \\
\hline Luxembourg & 12,6 & 16,5 & 14,3 & 18,5 & 15,5 & 20,0 & 17,4 & 21,0 & 9,0 & 10,7 \\
\hline Pays-Bas & 14,1 & 18,7 & 14,5 & 19,2 & 15,4 & 19,4 & 17,4 & 20,7 & 11,2 & 12,1 \\
\hline Royaume-Uni & 13,0 & 17,0 & 14,2 & 18,1 & 15,9 & 19,1 & 17,7 & 20,3 & 10,4 & 11,7 \\
\hline Suisse & 14,3 & 18,2 & 15,3 & 19,7 & 17,0 & 20,9 & 18,9 & 22,3 & - & - \\
\hline \multicolumn{11}{|l|}{ Europe du Sud } \\
\hline Albanie & - & - & 13,5 & 17,3 & 13,5 & 17,2 & - & - & - & - \\
\hline Bosnie-Herzégovine & - & - & 13,5 & 15,8 & - & - & - & - & - & - \\
\hline Chypre & 14,5 & 16,5 & 15,8 & 17,5 & 16,0 & 18,9 & 17,9 & 20,4 & 8,9 & 7,3 \\
\hline Croatie & 12,3 & 15,2 & 12,7 & 15,8 & 13,3 & 16,7 & 14,3 & 18,0 & - & - \\
\hline Espagne & 14,6 & 17,8 & 15,5 & 19,3 & 16,7 & 20,8 & 18,1 & 22,1 & 10,3 & 10,0 \\
\hline Grèce & 15,2 & 17,0 & 15,7 & 18,0 & 16,1 & 18,4 & 17,8 & 19,8 & 9,8 & 9,4 \\
\hline Italie & 13,9 & 17,4 & 15,2 & 19,0 & 16,7 & 20,7 & 18,2 & 22,0 & 7,9 & 7,3 \\
\hline Macédoine & - & - & - & - & 13,1 & 15,1 & 13,6 & 15,6 & - & - \\
\hline Malte & 10,7 & 12,8 & 15,4 & 18,0 & 15,1 & 18,5 & 17,0 & 20,1 & 10,4 & 11,3 \\
\hline Monténégro & - & - & 17,9 & 21,2 & 15,4 & 18,0 & 14,8 & 17,1 & - & - \\
\hline Portugal & 13,1 & 16,1 & 14,0 & 17,1 & 15,4 & 18,9 & 16,9 & 20,3 & 6,8 & 5,3 \\
\hline Serbie & 13,2 & 14,6 & 13,2 & 15,3 & 12,8 & 15,1 & 13,8 & 16,0 & - & - \\
\hline Slovénie & - & - & 13,4 & 17,1 & 14,2 & 18,7 & 16,4 & 20,5 & 9,0 & 9,9 \\
\hline \multicolumn{11}{|l|}{ Europe centrale } \\
\hline Bulgarie & 12,6 & 14,6 & 12,7 & 15,2 & 12,7 & 15,3 & 13,5 & 16,7 & - & - \\
\hline Hongrie & 11,6 & 14,7 & 12,0 & 15,4 & 13,0 & 16,8 & 13,9 & 18,1 & 5,3 & 5,8 \\
\hline Pologne & 12,0 & 15,5 & 12,4 & 16,2 & 13,6 & 17,5 & 14,8 & 19,1 & 6,5 & 7,0 \\
\hline Rép. tchèque & 12,6 & 14,2 & 13,3 & 15,3 & 13,5 & 16,0 & 14,0 & 17,2 & 7,6 & 7,7 \\
\hline Roumanie & 12,0 & 15,2 & 12,3 & 16,0 & 12,9 & 16,7 & 13,8 & 17,8 & 4,1 & 4,1 \\
\hline Slovaquie & 11,2 & 14,4 & 11,7 & 15,3 & 13,8 & 17,3 & 15,3 & 18,8 & 8,0 & 8,3 \\
\hline \multicolumn{11}{|l|}{ Europe de l'Est } \\
\hline Estonie & 11,8 & 15,6 & 12,0 & 15,8 & 12,6 & 17,0 & 13,6 & 18,9 & 3,5 & 4,1 \\
\hline Lettonie & 12,3 & 15,8 & 12,2 & 15,8 & 12,5 & 17,0 & 13,0 & 17,9 & 5,1 & 4,3 \\
\hline Lituanie & 13,4 & 16,6 & 13,3 & 17,0 & 13,7 & 17,9 & 13,4 & 18,1 & 5,2 & 5,4 \\
\hline Moldavie & 12,0 & 14,0 & 12,6 & 14,8 & 11,4 & 13,9 & 12,1 & 14,7 & - & - \\
\hline Russie & 11,6 & 15,6 & 12,1 & 15,9 & 11,1 & 15,2 & 11,8 & 16,1 & & \\
\hline États-Unis & 14,1 & 18,3 & 15,1 & 18,9 & 16,2 & 19,3 & $17,0^{*}$ & $19,7^{*}$ & & \\
\hline Canada & 14,5 & 18,9 & 15,7 & 19,9 & 16,8 & 20,4 & $18,2^{*}$ & $21,4^{*}$ & & \\
\hline
\end{tabular}


Tableau A.10. Proportions de 65 ans et plus, de 80 ans et plus (\%)

\begin{tabular}{|c|c|c|c|c|c|c|}
\hline & \multicolumn{3}{|c|}{65 ans et plus } & \multicolumn{3}{|c|}{80 ans et plus } \\
\hline & 1980 & 2008 & 2040 & 1980 & 2008 & 2040 \\
\hline \multicolumn{7}{|c|}{ Europe du Nord } \\
\hline Danemark & 14,3 & 15,6 & 24,8 & 2,8 & 4,1 & 8,1 \\
\hline Finlande & 11,9 & 16,5 & 26,2 & 1,7 & 4,3 & 10,1 \\
\hline Islande & 9,8 & 11,5 & - & 2,2 & 3,2 & - \\
\hline Norvège & 14,7 & 14,6 & 23,8 & 2,9 & 4,6 & 7,8 \\
\hline Suède & 16,2 & 17,5 & 24,3 & 3,1 & 5,3 & 8,4 \\
\hline \multicolumn{7}{|c|}{ Europe de l'Ouest } \\
\hline Allemagne & 15,7 & 20,1 & 31,1 & 2,6 & 4,6 & 10,3 \\
\hline Autriche & 15,5 & 17,2 & 27,2 & 2,6 & 4,6 & 8,4 \\
\hline Belgique & 14,3 & 17,0 & 25,0 & 2,6 & 4,7 & 8,4 \\
\hline France & 14,0 & 16,6 & 25,3 & 2,8 & 5,0 & 9,3 \\
\hline Irlande & 10,7 & 11,2 & 19,4 & 1,8 & 2,7 & 5,7 \\
\hline Luxembourg & 13,7 & 14,0 & 22,2 & 2,2 & 3,4 & 6,7 \\
\hline Pays-Bas & 11,5 & 14,7 & 26,9 & 2,2 & 3,8 & 9,0 \\
\hline Royaume-Uni & 14,9 & 16,1 & 22,4 & 2,7 & 4,5 & 7,3 \\
\hline Suisse & 13,8 & 16,4 & 26,1 & 2,6 & 4,7 & 8,7 \\
\hline \multicolumn{7}{|c|}{ Europe du Sud } \\
\hline Chypre & 10,7 & 12,5 & 20,0 & - & 2,8 & 6,1 \\
\hline Croatie & 11,0 & 17,2 & - & - & 3,2 & - \\
\hline Espagne & 10,8 & 16,6 & 27,7 & 1,7 & 4,6 & 8,3 \\
\hline Grèce & 13,1 & 18,6 & 28,4 & 2,3 & 4,1 & 8,9 \\
\hline Italie & 13,1 & 20,0 & 30,8 & 2,1 & 5,5 & 10,0 \\
\hline Macédoine & - & 11,4 & - & - & 1,7 & - \\
\hline Malte & 8,4 & 13,5 & 25,7 & 0,9 & 2,8 & 9,3 \\
\hline Monténégro & - & 12,9 & - & - & 2,0 & - \\
\hline Portugal & 11,2 & 17,4 & 26,8 & 1,6 & 2,5 & 8,4 \\
\hline Slovénie & 10,9 & 16,3 & 29,1 & 1,8 & 3,6 & 9,9 \\
\hline \multicolumn{7}{|c|}{ Europe centrale } \\
\hline Bulgarie & 11,8 & 17,3 & 26,7 & 1,5 & 3,6 & 8,0 \\
\hline Hongrie & 13,5 & 16,2 & 25,0 & 2,0 & 3,7 & 8,4 \\
\hline Pologne & 10,2 & 13,5 & 25,9 & 1,4 & 3,0 & 9,4 \\
\hline Rép. tchèque & 13,6 & 14,6 & 26,3 & 1,9 & 3,4 & 8,4 \\
\hline Roumanie & 10,3 & 14,9 & 25,5 & 1,2 & 2,8 & 7,4 \\
\hline Slovaquie & 10,6 & 12,0 & 25,3 & 1,5 & 2,6 & 7,8 \\
\hline \multicolumn{7}{|c|}{ Europe de l'Est } \\
\hline Estonie & 12,5 & 17,2 & 24,2 & 2,1 & 3,7 & 7,8 \\
\hline Lettonie & 13,0 & 17,3 & 25,4 & 2,3 & 3,5 & 7,9 \\
\hline Lituanie & 11,3 & 15,8 & 26,3 & 2,0 & 3,3 & 7,8 \\
\hline Russie & 10,2 & $12,9 *$ & 20,5 & 1,4 & 3,0 & 5,8 \\
\hline États-Unis & 11,2 & 13,0 * & 21,0 & 2,4 & 3,8 & 7,0 \\
\hline Canada & 9,4 & $14,1^{*}$ & 24,5 & 1,8 & 3,9 & 8,4 \\
\hline
\end{tabular}




\section{RÉFÉRENCES}

AVDEEV A., 2003, « On the way to one-child family: Are we beyond the point of no return? Some considerations concerning the fertility decrease in Russia », in Kotowska I. E., Jzwiak J. (eds.), Population of Central and Eastern Europe: Challenges and Opportunities, European Population Conference, Varsovie, 26-30 août, p. 139-163.

AvdeEV A., BLUM A., 1996, « La mortalité infantile dans les États issus de l'URSS », in Masuy-Stroobant G., Gourbin C., Buekens P. (dir.), Santé et mortalité des enfants en Europe, Louvain-la-Neuve, Chaire Quetelet 1994, Academia-Bruylant/L'Harmattan, p. 415-435.

Avdeev A., Blum A., Zakharov S., Andreev E., 1997, « Réaction d'une population hétérogène à une perturbation. Un modèle d'interprétation des évolutions de mortalité en Russie », Population, 52(1), p. 7-44.

BILLARI F. C., 2008, « Lowest-low fertility in Europe: Exploring the causes and finding some surprises », The Japanese Journal of Population, 6(1), p. 2-18.

Borrel C., Lhommeau B., 2010, « Être né en France d'un parent immigré », Insee Première, 1287, 4 p.

BOURDELAIS P., 1996, « Un seuil évolutif d'âge de la vieillesse : approches comparées France-Suède », Annales de démographie historique, p. 85-97.

Cambois E., Desesquelles A., Ravaud J.-F., 2003, « Femmes et hommes ne sont pas égaux face aux handicaps ", Population et sociétés, 386, 4 p.

CASElli G., VAllin J., 2002, "Les variations géographiques de la mortalité », in Caselli G., Vallin J., Wunsch G. (dir.), Démographie : analyse et synthèse. III. Les déterminants de la mortalité, Paris, Ined, p. 373-415.

CHOldin H., 1986, « Statistics and politics: The 'Hispanic issue' in the 1980 census », Demography, 23(3), p. 403-418.

COLEMAN D., 2006, " Immigration and ethnic change in low-fertility countries: A third demographic transition », Population and Development Review, 32(3), p. 401-446.

CORIJN M., KLiJZING E. (eds), 2001, Transitions to Adulthood in Europe, Boston, Kluwer Academic Publishers, 340 p.

Courbage Y., 2003, "Catholiques et protestants d'Irlande du Nord, les enjeux du recensement de $2001 »$, Population et sociétés, n 390, 4 p.

DAVIE E., MAZUY M., 2010, «Fécondité et niveau d'études des femmes en France à partir des enquêtes annuelles de recensement », Population 65(3), p. 475-512.

Dumont J.-C., Spielvogel G., 2008, A Profile of Immigrant Populations in the $21^{\text {st }}$ Century: Data from OECD Countries, Paris, OCDE.

EGGERICKX T., BÉGEOT F., 1993, « Les recensements en Europe dans les années 1990 : de la diversité des pratiques nationales à la comparabilité internationale des résultats ", Population, 48(6), p. 1705-1732.

EUROSTAT, http://epp.eurostat.ec.europa.eu/portal/page/portal/statistics/search_database

Fleury M., Henry L., 1956, Des registres paroissiaux à l'histoire de la population. Manuel de dépouillement et d'exploitation de l'état civil ancien, Paris, Ined, $84 \mathrm{p}$.

FREJKA T., CALOT G., 2001, « Cohort reproductive patterns in low-fertility countries », Population and Development Review, 27(1), p. 103-132. 
GAUthier A., 2007, "The impact of family policies on fertility in industrialized countries: A review of the literature », Population Research and Policy Review, 26(3), p. 323-346.

GAYMU J., EKAMPER P., BEETS G., 2007, « Qui prendra en charge les Européens âgés dépendants en 2030 ? », Population, 62(4), p. 789-822.

Gaymu J., Delbès C., Springer S., Binet A., Desesquelles A., Kalogirou S., ZIEGLER U., 2006, " Determinants of the living arrangements of older people in Europe », European Journal of Population, 22(3), p. 241-262.

GIANNAKOURIS K., 2008, « Ageing characterises the demographic perspectives of the European societies », Eurostat, Statistics in Focus, 72/2008.

GOLDSCHEIDER F., OLÁH L. S., PUUR A., 2010, « Reconciling studies of men's gender attitudes and fertility: Response to Westoff and Higgins », Demographic Research, 22, p. 189-198.

GOLL M., 2010, « Ageing in the European Union: Where exactly? », Eurostat, Statistics in Focus, 26/2010.

GOUBERT P., 1960, Beauvais et la Beauvaisis de 1600 à 1730, Paris, Sevpen, 653 p.

GUSTAFSON P., 2008, «Transnationalism in retirement migration: The case of North European retirees in Spain », Ethnic and Racial Studies, 31(3), p. 451-475.

HANTRAis L., 2009, International Comparative Research: Theory, Methods and Practice, Basingstoke / New York, Palgrave Macmillan and St Martin's Press, 208 p.

Harkness J. A., VAn De VijVer F. J. R., Mohler P. PH. (eds.), 2002, Cross-Cultural Survey Methods, Hoboken, New Jersey, John Wiley \& Sons, 432 p.

HENRY L., 1949, « Évolution démographique de l'Europe 1938-1947, d'après un article de Grzegorz Frumkin dans le «Bulletin économique pour l'Europe» », Population, 4(4), p. 743-748.

HENRY L., 1953, «Une richesse démographique en friche : les registres paroissiaux » Population, 8(2), p. 281-290.

HÉRAN F., PISON G., 2007, « Deux enfants par femme dans la France de 2006 : la faute aux immigrées? ", Population et sociétés, 432, 4 p.

HoEM J. M., 2008, « The impact of public policies on European fertility », Demographic Research, Special Collection 7: Childbearing Trends and Policies in Europe, 19, p. 249-260.

Hoffmeyer-Zlotnik J. H. P., Warner U., 2008, Private Household Concepts and their Operationalisation in National and International Social Surveys, Vol. 1, [Gesis Forschungsberichte, Reihe Survey Methodology], Mannheim, Gesis-Zuma, 138 p.

Hoffmeyer-Zlotnik J. H. P., WOlf C. (eds.), 2003, Advances in Cross-National Comparison: A European Working Book for Demographic and Socio-Economic Variables, New York, Kluwer Academic/Plenum Publishers, 419 p.

INGLEHART R., 1990, Culture Shift in Advanced Industrial Society, Princeton, Princeton University Press, $484 \mathrm{p}$.

INED, http://www.ined.fr/fr/pop_chiffres/pays_developpes/base_pays_developpes/

INSEE, 2005, Les immigrés en France - Édition 2005,

http://www.insee.fr/fr/publications-et-services/sommaire.asp?codesage $=i m m f r a 05$

Jagger C., Gillies C., Moscone F., Cambois E., Van Oyen H., Nusselder W., ROBINE J.-M., THE EHLEIS TEAM, 2008, « Inequalities in healthy life years in the 25 countries of the European Union in 2005: A cross-national meta-regression analysis *, The Lancet, 372(20/27), p. 2124-2131.

KAA D. J. VAN DE, 1987, « Europe's Second Demographic Transition », Population Bulletin, 42, p. 1-59. 
KAA D. J. VAN DE, 2003, «Second Demographic Transition », in Demeny P., McNicoll G. (eds.), Encyclopedia of Population, Macmillan Reference USA, New York, ThomsonGale, vol. 2, p. 872-875.

KinG R., WARNES A. M., Williams A. M., 1998, « International retirement migration in Europe ", International Journal of Population Geography, 4(2), p. 91-111.

KingKADE W. W., SAWYER C. C., 2001, « Infant mortality in Eastern Europe and the Former Soviet Union before and after the breakup », Meetings of the International Union for the Scientific Study of Population, Salvador de Bahia, Brazil, August 19-24.

Kupiszewska D., Kupiszewski M., Martí M., Ródenas C., 2010, Possibilities and Limitations of Comparative Quantitative Resarch on International Migration Flows Prominstat, Working paper $\mathrm{n}^{\circ}$ 4, $56 \mathrm{p}$.

LANDRY A., 1934, La révolution démographique. Études et essais sur les problèmes de la population, Paris, Ined, réédition de 1982, 227 p.

LeSTHAEGHE R., 2001, « Posptponement and recuperation: Recent fertility trends and forecasts in six Western European countries », IUSSP working group on low fertility seminar International Perspectives on Low Fertility, Tokyo, 21-23 March, 26 p.

LesthaEGHE R., KAA D. J. VAN DE, 1986, « Twee demografische transities? », [Deux transitions démographiques ?], in Lesthaeghe et van de Kaa (eds), Bevolking - Groei en Krimp, Mens en Maatschappij, Van Loghum Slaterus, Deventer, p. 9-24.

LESTHAEGHE R., SURKYN J., 2004, « Value orientations and the Second Demographic Transition (SDT) in Northern, Western and Southern Europe: An update », Demographic Research, Max Planck Institute for Demographic Research, Rostock, April 17, Special Collection 3, n 3, p. 45-86.

Letablier M.-T., LuCi A., MAth A., ThÉVEnOn O., 2009,The Costs of Raising Children and the Effectiveness of Policies to Support Parenthood in European Countries: A Literature Review. A report to the European Commission, 164 p.

MCDONALD P., 2008, «Very low fertility. Consequences, causes and policy approaches », The Japanese Journal of Population, 6(1), p. 19-23.

MESLÉ F., 2004, « Espérance de vie : un avantage féminin menacé ? », Population et sociétés, 402,4 p.

MESLÉ F., 2006, « Progrès récents de l'espérance de vie en France : les hommes comblent une partie de leur retard », Population, 61(4), p. 437-462.

Meslé F., VAllin J., 2002a, « Mortalité en Europe : la divergence Est-Ouest », Population, 57(1), p. 171-212.

MeSLÉ F., VAllin J., 2002b, « La transition sanitaire : tendances et perspectives », in Caselli G., Vallin J., Wunsch G. (dir.), Démographie : analyse et synthèse. III. Les déterminants de la mortalité, Paris, Ined, p. 439-461.

Mestheneos E., Triantafillou J., 2005, Supporting Family Carers of Older People in Europe: the Pan-European Background Report, University of Hamburg, H. Döhner \& C. Kofahl, vol. 1, p. 155.

Monnier A., 2006, Démographie contemporaine de l'Europe. Évolution, tendances, défis, Paris, Armand Colin, 416 p.

Monnier A., 2007, « Le baby-boom, suite et fin », Population et sociétés, 431.

Monnier A., Rychtarikova J., 1991, « Comment l'Europe s'est divisé entre l'Est et l'Ouest », Population, 46(6), p. 1617-1650.

NATIONS UNIES, 1998, Recommendations on Statistics of International Migration. Revision 1, New York, Nations unies.

NATIONS UNIES, 2009a, International Migration Report 2006: A Global Assessment, New York, Nations unies. 
NATIONS UNIES, 2009b, Trends in International Migrant Stock: The 2008 Revision, New York, United Nations database, POP/DB/MIG/Stock/Rev.2008).

NATIONS UNIES, 2009c, World Population Prospects: The 2008 Revision, New York, 89 p., http://esa.un.org/unpp

NiCOLAAS H., SPRANGERS A., 2004, « Immigrants come and go », Statistics Netherlands, Web magazine.

OBSERVATORIO PERMANENTE DE LA INMIGRACION (OPI), 2008, Anuario Estadistico de Inmigracion 2008.

OBSERVATORIO PERMANENTE DE LA INMIGRACION (OPI), 2009, Boletin Estadistico de Extranjeria e Inmigracion, $\mathrm{n}^{\circ} 19$.

OCDE, 2008, Perspectives des migrations internationales: SOPEMI 2008, OCDE.

OMRAN A. R., 1971, « The epidemiological transition: A theory of the epidemiology of population change », Milbank Memorial Fund Quarterly, 49(4), p. 509-538.

OstBy L., 2002, The Demographic Characteristics of Immigrant Populations in Norway, Technical report, Statistics Norway.

PADILla B., PEIXOTO J., 2007, Latin American Immigration to Southern Europe, Migration Policy Institute.

PISON G., 2010, « Le nombre et la part des immigrés dans la population : comparaisons internationales », Population et sociétés, n 472, 4 p.

RABINOWICZ L., 1929, Le problème de la population en France précédé d'une histoire générale de la population, Paris, Marcel Rivière, 429 p.

RØNSEN M., SKREDE K., 2010, « Can public policies sustain fertility in the Nordic countries? Lessons from the past and questions for the future », Demographic Research, vol. 22, p. 321-346.

SARDON J.-P., 2006, «Évolution démographique récente des pays développés », Population, 61(3), p. 225-300.

Shkolnikov V., Meslé F., VAllin J., 1995, « La crise sanitaire en Russie », Population, 50(4-5), p. 907-982.

SOBOTKA T., 2010, « Les migrants exercent-ils une influence croissante sur la fécondité en Europe? », Politiques sociales et familiales, $\mathrm{n}^{\circ} 100$ Fécondité et politiques publiques, p. 41-59.

SOBOTKA T., TOULEMON L., 2008, « Changing family and partnership behaviour: Common trends and persistent diversity across Europe », Demographic research, Special collection 7: Childbearing trends and Policies in Europe, vol. 19, p. 85-138.

SOMERVILle W., SRISKANDARAJAH D., LATORRE M., 2009, United Kingdom: A Reluctant Country of Immigration, Migration Policy Institute.

THÉVENON O., 2008, «Les politiques familiales des pays développés : des modèles contrastés », Population et sociétés, $\mathrm{n}^{\circ} 448,4$ p.

THIERry X., 2008, « Migrations : le défi statistique européen », Futuribles, 343, p. 61-77.

ThORnTON A., PHilipov D., 2009, « Sweeping changes in marriage, cohabitation and childbearing in Central and Eastern Europe: New insights from the developmental idealism framework », European Journal of Population, 25(2), p. 123-156.

TOUlEMON L., MAZUY M., 2004, Comment prendre en compte l'âge à l'arrivée et la durée de séjour en France dans la mesure de la fécondité des immigrants ?, Paris, Ined, Documents de travail $\mathrm{n}^{\circ} 120,34 \mathrm{p}$.

TRIBALAT M., 2005, «Fécondité des immigrées et apport démographique de l'immigration étrangère », in Bergouignan C., Blayo C., Parant A., Sardon J.-P., Tribalat M., La population de la France: évolutions démographiques depuis 1946, Cudep, p. $727-767$. 
TRIBALAT M., 2008, «Effets démographiques de l'immigration étrangère. Éléments de comparaison européenne », Futuribles, 343, p. 41-60.

VALENTE P., 2010, « Comment la population est-elle recensée dans les pays européens en 2010 ? », Populations et sociétés, n 467, 4 p.

VALlin J., MeSLÉ F., 2010, «Espérance de vie : peut-on gagner trois mois par an indéfiniment? », Population et sociétés, n 473, 4 p.

Van Oyen H., Cox B., Jagger C., Cambois E., Nusselder W., Gillies C., Robine J.-M., 2010, « Gender gaps in life expectancy and expected years with activity limitations at age 50 in the European Union: Associations with macro-level structural indicators ", European Journal of Ageing , 7, p. 229-237.

VASILEVA K., 2009, «Citizens of European countries account for the majority of the foreign population in EU-27 in 2008 », Eurostat, Statistics in Focus, 94.

VIGOUR C., 2005, La comparaison dans les sciences sociales, Paris, La Découverte, 336 p.

Vila M. R., MARTíN T. C., 2007, « La fécondité des étrangères dans un pays d'immigration récente : le cas de l'Espagne ", Population, 63(3), p. 419-450.

VOLLSET S. E., 2008, « Baisse générale de la mortalité adulte en Europe de l'Ouest : les Espagnoles et les Suédoises tiennent la tête ", Population et sociétés, n 450, 4 p.

ZLOTNIK H., 1995, « The south-to-north migration of women », International Migration Review, 29(1), p. 229-254. 


\section{Alexandre Avdeev, Tatiana ERemenko, Patrick Festy, Joëlle Gaymu, Nathalie Le BOUTEILLEC, Sabine SPRINGER • POPULATIONS ET TENDANCES DÉMOGRAPHIQUES DES PAYS EUROPÉENS (1980-2010)}

Au cours des trente dernières années, la croissance de la population européenne s'est effectuée à un rythme de plus en plus ralenti. L'excédent naturel des naissances sur les décès n'a cessé de reculer, et l'excédent migratoire représente désormais un apport substantiel, parfois décisif pour le maintien d'une croissance positive. Cette tendance générale est largement partagée par la plupart des pays, à travers un mouvement qui combine recul de la fécondité, progression des espérances de vie et solde migratoire positif en augmentation. À un niveau plus fin d'analyse, on observe une évolution semblable des formes familiales vers moins de mariages et davantage de situations de fait, et un même recul des causes majeures de mortalité, cancéreuses ou cardiovasculaires. Pourtant, il y a moins convergence de ces tendances à travers l'espace européen que répétition à quelques années d'intervalle de ces transformations, les écarts se maintenant entre les pays ou les régions du continent. L'avenir de la population européenne dépendra largement du niveau de la fécondité future : croissance ou décroissance selon qu'elle sera élevée ou faible, quasi-stabilité si elle reste moyenne. Quelle que soit la fécondité des quarante ans à venir, le vieillissement de la population européenne est inéluctable, y compris en cas de poursuite de l'immigration, et des différences notables vont perdurer à travers l'Europe.

\section{Alexandre Avdeev, Tatiana EREMENKo, Patrick Festy, Joëlle GAYMU, Nathalie Le BOUTEILLEC, Sabine SPRINGER • POPULATIONS AND DEMOGRAPHIC TRENDS OF EUROPEAN COUNTRIES (1980-2010)}

European population growth has slowed over the last thirty years, with a steadily decreasing excess of births over deaths. Net migration is now a major contributor and in some countries plays a decisive role in maintaining positive population growth. This general trend is common to most European countries, reflecting the combined effects of fertility decline, higher life expectancy, and positive and increasing net migration. At a more detailed level of analysis, the countries of Europe exhibit similar trends in family transformation, with fewer marriages and more informal unions, and in the major causes of death, with decreases in cardiovascular and cancer mortality. However, rather than a convergence of these trends across Europe, we are witnessing a repetition of similar changes at intervals of several years, with the gaps between countries or sub-regions remaining largely unchanged. The future of the European population will depend largely on fertility in coming years, with growth if fertility is high, decline if it is low, and relative stability if it remains at moderate levels. Whatever the level of fertility in the next forty years, European population ageing will be inevitable, even if immigration remains at current levels, and substantial differences across Europe will persist.

\section{Alexandre Avdeev, Tatiana ERemenko, Patrick Festy, Joëlle GAYMu, Nathalie Le BOUTEILLEC, Sabine SPRINGER • POBLACIONES Y TENDENCIAS DEMOGRÁFICAS DE LOS \\ PAÍSES EUROPEOS (1980-2010)}

La lentificación del crecimiento de la población europea se ha proseguido durante los últimos treinta años. El excedente natural de los nacimientos sobre las defunciones no ha cesado de disminuir, y el excedente migratorio representa ahora un aporte substancial, a veces decisivo para el mantenimiento de un crecimiento positivo. Esta tendencia general es común a la mayor parte de los países, a través de un movimiento que combina el retroceso de la fecundidad, la progresión de la esperanza de vida y un saldo migratorio positivo en aumento. Afinando el análisis, se observa una evolución similar de las formas familiares con una menor frecuencia de los matrimonios y una mayor de las parejas de hecho, así como un retroceso común de las principales causas de mortalidad, por cáncer o por enfermedad cardiovascular. Sin embargo, más que una convergencia de tendencias a través del espacio europeo, se observa de hecho una repetición de dichas transformaciones a varios años de intervalo, lo que mantiene las distancias entre los países o las regiones del continente. El porvenir de la población europea dependerá ampliamente del nivel de la fecundidad: crecimiento o decrecimiento según que este nivel sea elevado o débil, cuasi-estabilidad si es mediano. Cualquiera que sea la fecundidad en los cuarenta próximos años, el envejecimiento de la población europea es inevitable, incluso si la inmigración continúa, y diferencias notables van a perdurar a través de Europa.

Mots-clés : Europe, croissance démographique, fécondité, mortalité, migration, vieillissement, projection.

Keywords: Europe, population growth, fertility, mortality, migration, ageing, projection. 
